\title{
On $\mathrm{C}^{*}$-algebras associated to product systems
}

\author{
Dissertation \\ zur Erlangung des mathematisch-naturwissenschaftlichen Doktorgrades \\ „Doctor rerum naturalium“ (Dr.rer.nat.) \\ der Georg-August-Universität Göttingen \\ im Promotionsprogramm der PhD School of Mathematical Science (SMS) \\ der Georg-August University School of Science (GAUSS)
}

vorgelegt von

Camila Fabre Sehnem

aus Criciúma, Brasilien

Göttingen 2018 


\title{
Betreuungsausschuss
}

\author{
Betreuer: Prof. Dr. Ralf Meyer \\ Mathematisches Institut, Georg-August-Universität Göttingen \\ Betreuer: Prof. Dr. Chenchang Zhu \\ Mathematisches Institut, Georg-August-Universität Göttingen
}

\section{Mitglieder der Prüfungskommission}

\author{
Referent: Prof. Dr. Ralf Meyer \\ Mathematisches Institut, Georg-August-Universität Göttingen \\ Koreferent: Prof. Dr. Alcides Buss \\ Department of Mathematics, Federal University of Santa Catarina, Brazil
}

Prof. Dr. Christoph Lehrenfeld

Institut für Numerische und Angewandte Mathematik, Georg-August-Universität Göttingen

Prof. Dr. Ingo Witt

Mathematisches Institut, Georg-August-Universität Göttingen

Prof. Dr. Karl-Henning Rehren

Institut für Theoretische Physik, Georg-August-Universität Göttingen

Prof. Dr. Thomas Schick

Mathematisches Institut, Georg-August-Universität Göttingen

Tag der mündlichen Prüfung: 04.05.2018 


\section{Abstract}

We consider a class of Fell bundles over quasi-lattice ordered groups. We show that these are completely determined by the positive fibres and that their cross sectional $\mathrm{C}^{*}$-algebras are relative Cuntz-Pimsner algebras associated to simplifiable product systems of Hilbert bimodules. Conversely, we show that such product systems can be naturally extended to Fell bundles and this correspondence is part of an equivalence between bicategories. We also relate amenability for this class of Fell bundles to amenability of quasi-lattice orders by showing that Fell bundles extended from free semigroups are amenable. A similar result is proved for Baumslag-Solitar groups. Moreover, we construct a relative Cuntz-Pimsner algebra of a compactly aligned product system as a quotient of the associated Nica-Toeplitz algebra. We show that this construction yields a reflector from a bicategory of compactly aligned product systems into its sub-bicategory of simplifiable product systems of Hilbert bimodules. We use this to study Morita equivalence between relative Cuntz-Pimsner algebras.

In a second part, we let $P$ be a unital subsemigroup of a group $G$. We propose an approach to $\mathrm{C}^{*}$-algebras associated to product systems over $P$. We call the $\mathrm{C}^{*}$-algebra of a given product system $\mathcal{E}$ its covariance algebra and denote it by $A \times{ }_{\mathcal{E}} P$, where $A$ is the coefficient $\mathrm{C}^{*}$-algebra. We prove that our construction does not depend on the embedding $P \hookrightarrow G$ and that a representation of $A \times \mathcal{E} P$ is faithful on the fixed-point algebra for the canonical coaction of $G$ if and only if it is faithful on $A$. We compare this with other constructions in the setting of irreversible dynamical systems, such as Cuntz-Nica-Pimsner algebras, Fowler's Cuntz-Pimsner algebra, semigroup $\mathrm{C}^{*}$-algebras of Xin Li and Exel's crossed products by interaction groups. 


\section{Acknowledgements}

First of all, I am taking this opportunity to thank my supervisor, Prof. Dr. Ralf Meyer, for his guidance and support. I am also grateful to him for accepting me as his student.

I am grateful to Prof. Dr. Alcides Buss who agreed to be the second referee for my thesis. I am also thankful to Prof. Dr. Alcides Buss and Prof. Dr. Ruy Exel who helped me to come to Germany.

I am grateful to my colleagues and friends from the Mathematisches Institut - Göttingen for coffee and lunch time.

I especially thank my parents, my sister and my brother for their love and for always being there for me.

I am also grateful to my close friends from Göttingen for many special moments. And, of course, a very special thanks to Alex and Marcelo who have been my family in Germany since my first days in Göttingen.

I am deeply thankful to the financial support of CNPq (National Council for Scientific and Technological Development) to obtain a PhD in mathematics from Germany. I thank DAAD (Deutscher Akademischer Austausch Dienst) for providing the financial support to an intensive German language course. I also thank Prof. Ralf Meyer for covering my travel expenses to conferences from his grant. 



\section{Contents}

1 Introduction 1

1.1 Motivation: irreversible dynamical systems $\ldots \ldots \ldots \ldots \ldots \ldots$

$1.2 \quad \mathrm{C}^{*}$-algebras associated to correspondences $\ldots \ldots \ldots \ldots \ldots \ldots \ldots$

1.3 Compactly aligned product systems $\ldots \ldots \ldots \ldots \ldots \ldots \ldots$

1.4 Fell bundles over quasi-lattice ordered groups $\ldots \ldots \ldots \ldots$. . . . . . . . . . . 4

1.5 Bicategories of correspondences $\ldots \ldots \ldots \ldots \ldots \ldots \ldots \ldots$

1.6 Covariance algebras of product systems $\ldots \ldots \ldots \ldots \ldots \ldots$

\begin{tabular}{|ll}
\hline 2 Fell bundles and coactions & 10
\end{tabular}

2.1 Fell bundles . . . . . . . . . . . . . . . . . . . . . . . . . . . . 10

$2.1 .1 \quad$ Cross sectional $\mathrm{C}^{*}$-algebra $\ldots \ldots \ldots \ldots \ldots$

2.1 .2 The regular representation. . . . . . . . . . . . . . . . . . . . 12

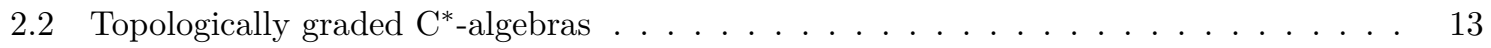

$2.2 .1 \quad$ Conditional expectation $\ldots \ldots \ldots \ldots \ldots \ldots$

$2.2 .2 \quad$ Discrete coactions $\ldots \ldots \ldots \ldots \ldots \ldots \ldots \ldots$

\begin{tabular}{|lll}
\hline A bicategorical interpretation for relative Cuntz-Pimsner algebras & 16
\end{tabular}

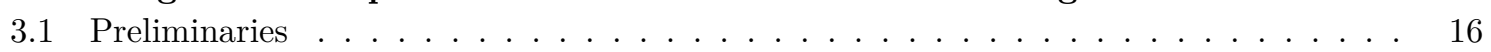

$3.1 .1 \quad$ Correspondences . . . . . . . . . . . . . . . . . . . . . . . . . 16

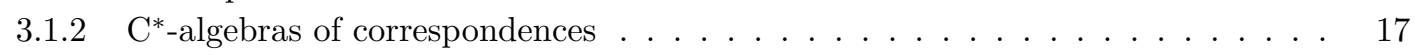

3.1 .3 Gauge action and Fell bundle structure . . . . . . . . . . . . . . . 20

3.1.4 Functoriality of relative Cuntz-Pimsner algebras . . . . . . . . . . . . . 21

$3.2 \quad$ Bicategories of correspondences and Hilbert bimodules . . . . . . . . . . . . . . . . . 23

3.3 The reflector from correspondences to Hilbert bimodules . . . . . . . . . . . . . . 26

$\begin{array}{|ll|}4 \text { Product systems and some } \mathrm{C}^{*} \text {-constructions } & 31\end{array}$

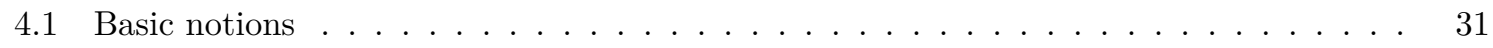

4.1 .1 Toeplitz algebras $\ldots \ldots \ldots \ldots \ldots \ldots \ldots$

4.1 .2 Fock representation $\ldots \ldots \ldots \ldots \ldots \ldots \ldots$

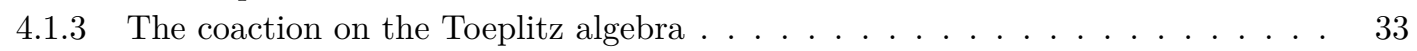

4.2 Compactly aligned product systems $\ldots \ldots \ldots \ldots \ldots \ldots \ldots \ldots$

$4.2 .1 \quad$ Nica-Toeplitz algebras . . . . . . . . . . . . . . . . . . . . 34

$4.2 .2 \quad$ Relative Cuntz-Pimsner algebras . . . . . . . . . . . . . . . . . . 35

$4.2 .3 \quad$ Coaction on relative Cuntz-Pimsner algebras $\ldots \ldots \ldots$. . . . . . . . 38

4.3 Bicategories of compactly aligned product systems $\ldots \ldots \ldots \ldots \ldots$

\begin{tabular}{|lll}
5 & Fell bundles over quasi-lattice ordered groups & 42
\end{tabular}

5.1 From product systems of Hilbert bimodules to Fell bundles . . . . . . . . . . . . . . 42

5.2 The equivalence of bicategories $\ldots \ldots \ldots \ldots \ldots \ldots \ldots \ldots$

5.3 Amenability for Fell bundles extended from free semigroups $\ldots \ldots \ldots \ldots \ldots$. . . . . 54

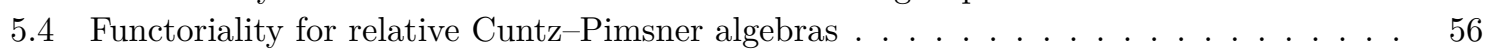

5.4 .1 Relative Cuntz-Pimsner algebras as universal arrows . . . . . . . . . . . 56

5.4 .2 Morita equivalence for relative Cuntz-Pimsner algebras . . . . . . . . . . . . 59 
$6 \quad \mathrm{C}^{*}$-algebras for product systems over subsemigroups of groups 6

6.1 Strongly covariant representations . . . . . . . . . . . . . . . . . 65

6.2 Covariance algebras associated to product systems . . . . . . . . . . . . . . . . . . . . 68

6.3 Relationship to other constructions . . . . . . . . . . . . . . . . . . . . . 70

6.3.1 Relationship to a construction by Sims and Yeend . . . . . . . . . . . . . . . 71

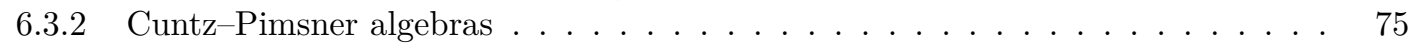

6.3 .3 Semigroup $\mathrm{C}^{*}$-algebras . . . . . . . . . . . . . . . . . . . . . . 76

6.3 .4 Crossed products by interaction groups $\ldots \ldots \ldots \ldots$. . . . . . . . . . . 78

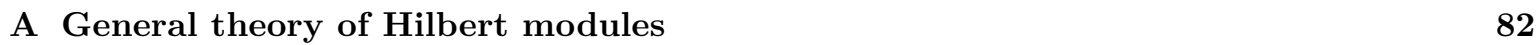

A.1 Adjointable operators on Hilbert modules . . . . . . . . . . . . . . . . . . . . . . . . . 82

A.2 Morita equivalence . . . . . . . . . . . . . . . . . . . . 83

\begin{tabular}{lr}
\hline B Bicategories & $\mathbf{8 6}$
\end{tabular}

B.1 Bicategories, homomorphisms and transformations . . . . . . . . . . . . . . . . . 86 


\section{Chapter 1}

\section{Introduction}

This introduction explains the context of this thesis. Our main results are stated in Sections 1.4, 1.5 and 1.6. We have included here the statements of a few theorems, propositions as well as corollaries that are our own results. Further results from this thesis mentioned in this introduction are highlighted with italics.

\subsection{Motivation: irreversible dynamical systems}

The $\mathrm{C}^{*}$-algebra generated by the image of a homomorphism $U: \mathbb{Z} \rightarrow \mathrm{U}(\mathcal{H})$ from the integers into the group of unitaries of a Hilbert space is just the closed *-subalgebra of $\mathbb{B}(\mathcal{H})$ generated by the single unitary $U_{1}$. Hence the universal $\mathrm{C}^{*}$-algebra for unitary representations of $\mathbb{Z}$ is the universal $\mathrm{C}^{*}$-algebra generated by a single unitary. This is the $\mathrm{C}^{*}$-algebra $\mathrm{C}(\mathbb{T})$ of continuous functions on the unit circle $\mathbb{T}$ with the inclusion function $z: \mathbb{T} \rightarrow \mathbb{C}$ as the unitary generator.

The group $\mathrm{C}^{*}$-algebra $\mathrm{C}^{*}(G)$ of a discrete group $G$ is defined to be the universal $\mathrm{C}^{*}$-algebra for unitary representations of $G$. Every discrete group $G$ has a canonical unitary representation $\lambda$ on the Hilbert space $\ell_{2}(G)$, called left regular representation. This induces a *-homomorphism $\Lambda: \mathrm{C}^{*}(G) \rightarrow$ $\mathbb{B}\left(\ell_{2}(G)\right)$ by universal property. The reduced group $\mathrm{C}^{*}$-algebra $\mathrm{C}_{r}^{*}(G)$ is the image of $\mathrm{C}^{*}(G)$ in $\mathbb{B}\left(\ell_{2}(G)\right)$ under $\Lambda$. The regular representation produces an isomorphism $\mathrm{C}^{*}(G) \cong \mathrm{C}_{r}^{*}(G)$ if and only if $G$ is amenable with respect to the usual definition of amenability in terms of existence of a left invariant mean on $\ell_{\infty}(G)$.

If $X$ is a locally compact space, a ${ }^{*}$-automorphism of $\mathrm{C}_{0}(X)$ is equivalent to a homeomorphism of $X$. Given a *-automorphism of a $\mathrm{C}^{*}$-algebra $A$, a covariant representation $(\pi, U)$ of $(A, \mathbb{Z}, \alpha)$ is a *-homomorphism $A \rightarrow \mathbb{B}(\mathcal{H})$ together with a unitary $U$ in $\mathrm{U}(\mathcal{H})$ such that $\pi(\alpha(a))=U \pi(a) U^{*}$ for all $a$ in $A$, where $\mathcal{H}$ is a Hilbert space. The crossed product $A \rtimes_{\alpha} \mathbb{Z}$ is the universal $\mathrm{C}^{*}$-algebra for covariant representations of $(A, \mathbb{Z}, \alpha)$. Any faithful representation of $A$ on a Hilbert space $\mathcal{H}$ induces a canonical regular representation $(\pi, U)$ of $(A, \mathbb{Z}, \alpha)$ in $\mathbb{B}\left(\ell_{2}(\mathbb{Z}, \mathcal{H})\right)$ with $\pi$ injective. The corresponding representation of $A \rtimes_{\alpha} \mathbb{Z}$ is also injective. Since every $\mathrm{C}^{*}$-algebra can be faithfully represented on a Hilbert space, it follows that $A$ embeds into the crossed product $A \rtimes_{\alpha} \mathbb{Z}$.

A $\mathrm{C}^{*}$-dynamical system $(A, G, \alpha)$, or simply a dynamical system, consists of a $\mathrm{C}^{*}$-algebra $A$, a discrete group $G$ and a group homomorphism $\alpha$ from $G$ to the group of *automorphisms of $A$. Given a dynamical system, one can form the full and reduced crossed product $\mathrm{C}^{*}$-algebras. The first one, denoted by $A \rtimes_{\alpha} G$, is a universal object. It is the universal $\mathrm{C}^{*}$-algebra for covariant representations of $(A, G, \alpha)$. Here a covariant representation of $(A, G, \alpha)$ is a pair $(\pi, U)$, where $\pi$ is a representation of $A$ on a Hilbert space $\mathcal{H}$ and $U: G \rightarrow \mathrm{U}(\mathcal{H})$ is a unitary representation, such that

$$
\pi\left(\alpha_{g}(a)\right)=U_{g} \pi(a) U_{g}^{*}
$$

for all $a \in A$ and $g \in G$. The reduced crossed product $A \rtimes_{\alpha, r} G$, in turn, has a co-universal property. It is the smallest $\mathrm{C}^{*}$-algebra for a certain class of covariant representations. It is concretely defined through a regular representation of $(A, G, \alpha)$ in $\mathbb{B}\left(\ell_{2}(G, \mathcal{H})\right)$, obtained as above from a faithful representation of $A$ on $\mathcal{H}$. The corresponding *-homomorphism $A \rightarrow \mathbb{B}\left(\ell_{2}(G, \mathcal{H})\right)$ is injective, so that $A$ embeds into both the full and reduced crossed product $\mathrm{C}^{*}$-algebras. If $G$ is amenable, the regular representation induces an isomorphism $A \rtimes_{\alpha} G \cong A \rtimes_{\alpha, r} G$. 
Many technical issues arise when one tries to associate a $\mathrm{C}^{*}$-algebra to a single endomorphism of a $\mathrm{C}^{*}$-algebra, so that it encodes the dynamics as in the above $\mathrm{C}^{*}$-constructions for reversible dynamical systems. The usual notion of a covariant representation of $(A, \mathbb{N}, \alpha)$ consists of a representation of $A$ on a Hilbert space $\mathcal{H}$ and an isometry $v$ in $\mathbb{B}(\mathcal{H})$ satisfying the covariance condition

$$
\pi(\alpha(a))=v \pi(a) v^{*}
$$

for all $a \in A$. However, 11.1.1 forces the image of ker $\alpha$ in the resulting crossed product to be trivial. In addition, if $A$ is unital and $\alpha$ is a unital endomorphism, the underlying isometry of a nondegenerate covariant representation of $(A, \mathbb{N}, \alpha)$ will be a unitary, and one cannot expect it to have many covariant representations unless $\alpha$ is a ${ }^{*}$-automorphism. At this point, a different approach is needed to treat irreversible dynamical systems.

\section{2 $\quad \mathrm{C}^{*}$-algebras associated to correspondences}

A Hilbert $\mathrm{C}^{*}$-module is a generalisation of Hilbert spaces. In this more general setting, the (right) inner product takes values in a $\mathrm{C}^{*}$-algebra $A$ and is $A$-linear in the second variable. Thus a Hilbert space is a Hilbert $\mathbb{C}$-module. A correspondence $\mathcal{E}: A \leadsto B$ between $\mathrm{C}^{*}$-algebras $A$ and $B$ consists of a right Hilbert $B$-module with a nondegenerate left action of $A$ implemented by a ${ }^{*}$-homomorphism $\varphi: A \rightarrow \mathbb{B}(\mathcal{E})$, where $\mathbb{B}(\mathcal{E})$ is the $\mathrm{C}^{*}$-algebra of adjointable operators on $\mathcal{E}$. It is called faithful if $\varphi$ is injective. If $\mathcal{E}$ comes equipped with a left $A$-valued inner product so that $\varphi(\langle\langle\xi \mid \eta\rangle\rangle) \zeta=\xi\langle\eta \mid \zeta\rangle$ for all $\xi, \eta$ and $\zeta$ in $\mathcal{E}$, then we say that $\mathcal{E}$ is a Hilbert $A, B$-bimodule. A Hilbert $A, B$-bimodule $\mathcal{E}$ induces an adjoint Hilbert $B, A$-bimodule $\mathcal{E}^{*}$.

A celebrated construction by Pimsner associates a $\mathrm{C}^{*}$-algebra $\mathcal{O}_{\mathcal{E}}$ to a not necessarily invertible faithful correspondence $\mathcal{E}: A \leadsto A$ [8]. This is now known as a Cuntz-Pimsner algebra. It is the universal $\mathrm{C}^{*}$-algebra for representations of $\mathcal{E}$ that satisfy a certain condition, now called Cuntz-Pimsner covariance, on the ideal $J=\varphi^{-1}(\mathbb{K}(\mathcal{E})) \triangleleft A$. Here $\mathbb{K}(\mathcal{E})$ is the ideal of $\mathbb{B}(\mathcal{E})$ generated by all generalised rank-1 operators on $\mathcal{E}$, that is, the $\mathrm{C}^{*}$-algebra of compact operators on $\mathcal{E}$. Pimsner's $\mathrm{C}^{*}$-algebra includes many interesting $\mathrm{C}^{*}$-algebras, such as crossed products by automorphisms and graph $\mathrm{C}^{*}$-algebras for graphs with no sinks 30 . It also covers crossed products by extendible and injective endomorphisms with hereditary range. The Cuntz-Pimsner algebra $\mathcal{O}_{\mathcal{E}}$ is a quotient of a universal object, namely the Toeplitz algebra $\mathcal{T}_{\mathcal{E}}$. This latter $\mathrm{C}^{*}$-algebra associated to $\mathcal{E}$ was defined by Pimsner as the universal $\mathrm{C}^{*}$-algebra for representations of $\mathcal{E}$.

For a unital $\mathrm{C}^{*}$-algebra $A$ and an endomorphism $\alpha: A \rightarrow A$, the work of Exel in [21] suggests that one should be given a transfer operator $L$ for $(A, \alpha)$ in order to construct a reasonable crossed product. In fact, he was also inspired by Pimsner's $\mathrm{C}^{*}$-construction because there is a correspondence $A_{\alpha, L}$ over $A$ naturally associated to $(A, \alpha, L)$. Indeed, a continuous linear map $L: A \rightarrow A$ is a transfer operator if it is positive and $L(a \alpha(b))=L(a) b$ for all $a, b$ in $A$. This gives rise to a correspondence $A_{\alpha, L}: A \leadsto A$ obtained after dividing out a null-space and taking the completion from the subspace $A \alpha(1)$, under the following structure: the left action is given by the multiplication on $A$, the right action is implemented by $\alpha$ and the pre-inner product is defined by $\langle a \alpha(1) \mid b \alpha(1)\rangle=L\left(a^{*} b\right)$. Exel's $\mathrm{C}^{*}$-algebra $\mathcal{T}(A, \alpha, L)$ is precisely the Toepliz algebra of $A_{\alpha, L}$. In addition, his notion of redundancy corresponds to CuntzPimsner covariance for $A_{\alpha, L}$. So in case $A_{\alpha, L}$ is a faithful correspondence, the crossed product $A \rtimes_{\alpha, L} \mathbb{N}$ introduced in 21] coincides with the Cuntz-Pimsner algebra $\mathcal{O}_{\mathcal{E}}$ as defined in [48. Since Pimsner's work was devoted to faithful correspondences, it was unclear in general when $A$ embeds into the crossed product $A \rtimes_{\alpha, L} \mathbb{N}$.

For a non-faithful correspondence $\mathcal{E}$, Pimsner's $\mathrm{C}^{*}$-algebra may be zero. Muhly and Solel proposed a construction of $\mathrm{C}^{*}$-algebras associated to (not necessarily faithful) correspondences by taking universal $\mathrm{C}^{*}$-algebras for representations satisfying the covariance condition only on an ideal $J \triangleleft A$ with $J \subseteq \varphi^{-1}(\mathbb{K}(\mathcal{E}))[41$. In [29], Katsura provided necessary and sufficient conditions on the ideal $J$ for the universal representation of $\mathcal{E}$ in $\mathcal{O}_{J, \mathcal{E}}$ to be injective. Inspired by graph $\mathrm{C}^{*}$-algebras, among other constructions, he analysed the relative Cuntz-Pimsner algebra $\mathcal{O}_{J_{\mathcal{E}, \mathcal{E}}}$ with $J_{\mathcal{E}}:=(\operatorname{ker} \varphi)^{\perp} \cap \varphi^{-1}(\mathbb{K}(\mathcal{E})$ ). This $\mathrm{C}^{*}$-algebra has nice properties. First, the universal representation of $\mathcal{E}$ in $\mathcal{O}_{J_{\mathcal{E}}, \mathcal{E}}$ is injective. Hence it encodes the correspondence structure of $\mathcal{E}$. In addition, $\mathcal{O}_{J_{\mathcal{E}} \mathcal{E}}$ satisfies the gauge-invariant uniqueness theorem, which asserts that a representation of $\mathcal{O}_{J_{\mathcal{E}}, \mathcal{E}}$ in a $\mathrm{C}^{*}$-algebra $B$ that is faithful on the coefficient algebra $A$ is also faithful on $\mathcal{O}_{J_{\mathcal{E}}, \mathcal{E}}$, at least when the representation in question satisfies a certain compatibility condition. 


\section{INTRODUCTION}

In [11, Brownlowe and Raeburn then described the crossed product by an endomorphism $\alpha: A \rightarrow A$ of a unital $\mathrm{C}^{*}$-algebra relative to a transfer operator $L$ as a relative Cuntz-Pimsner algebra. With this interpretation at hand, they provided necessary and sufficient conditions for the universal representation in $A \rtimes_{\alpha, L} \mathbb{N}$ to be injective. They also applied gauge-invariant uniqueness theorems of relative CuntzPimsner algebras for Katsura's ideal to prove uniqueness theorems for the crossed product. So relative Cuntz-Pimsner algebras associated to correspondences turn out to be a very important tool to construct and study $\mathrm{C}^{*}$-algebras out of irreversible dynamical systems.

\subsection{Compactly aligned product systems}

Roughly speaking, a product system may be regarded as an action of a semigroup by correspondences over a $\mathrm{C}^{*}$-algebra. A product system over a semigroup $P$ with unit element denoted by $e$ is a family of correspondences $\mathcal{E}=\left(\mathcal{E}_{p}\right)_{p \in P}$ with $\mathcal{E}_{e}=A$ together with correspondence isomorphisms $\mathcal{E}_{p} \otimes_{A} \mathcal{E}_{q} \cong \mathcal{E}_{p q}$ subject to certain axioms. Product systems were introduced in this context by Fowler in [26], following the work of Arveson on continuous product systems of Hilbert spaces developed in [7]. As for single correspondences, examples of product systems arise naturally from semigroups of endomorphisms 26, 38.

Fowler defined the Toeplitz algebra $\mathcal{T}_{\mathcal{E}}$ of a given product system $\mathcal{E}$ as the universal $\mathrm{C}^{*}$-algebra for representations of $\mathcal{E}$, thus generalising Toeplitz algebras of single correspondences. Following Pimsner, he constructed the Cuntz-Pimsner algebra of a product system $\mathcal{E}=\left(\mathcal{E}_{p}\right)_{p \in P}$ as the universal $\mathrm{C}^{*}$-algebra for representations that are Cuntz-Pimsner covariant on $J_{p}=\varphi_{p}^{-1}\left(\mathbb{K}\left(\mathcal{E}_{p}\right)\right)$ for all $p \in P$. As in Pimsner's original construction, Fowler's Cuntz-Pimsner algebra might be trivial if $\mathcal{E}$ is non-faithful.

Unlike the case of single correspondences, the Toeplitz algebra of a product system is in general too big. For example, the universal $\mathrm{C}^{*}$-algebra for representations of the trivial bundle over $\mathbb{N} \times \mathbb{N}$ is not nuclear ${ }^{1}$ (see 45$\left.]\right)$. This is precisely the universal $C^{*}$-algebra generated by two commuting isometries. So in order to define a reasonable universal object, one must impose additional conditions on the representations of the underlying product system.

Nica considered a class of isometric representations of a semigroup arising from a quasi-lattice order 47]. This is a semigroup $P$ contained in a group, say $P \subseteq G$, so that the structure of the pair $(G, P)$ resembles that of $\left(\mathbb{Z}^{k}, \mathbb{N}^{k}\right)$. More precisely, we have $P \cap P^{-1}=\{e\}$ and, with respect to the partial order $g_{1} \leq g_{2} \Leftrightarrow g_{1}^{-1} g_{2} \in P$, two elements $g_{1}$ and $g_{2}$ in $G$ with a common upper bound in $P$ also have a least upper bound $g_{1} \vee g_{2}$ in $P$. We say that $(G, P)$ is a quasi-lattice ordered group. The relations imposed by Nica on the representations of $P$ arise naturally from its canonical representation by isometries in $\mathbb{B}\left(\ell_{2}(P)\right)$. The universal $\mathrm{C}^{*}$-algebra for such representations, denoted by $\mathrm{C}^{*}(G, P)$, is now known as the Toeplitz algebra of $(G, P)$. Nica also introduced a notion of amenability for quasi-lattice ordered groups by saying that $(G, P)$ is amenable if the regular representation of $\mathrm{C}^{*}(G, P)$ on $\ell_{2}(P)$ is faithful. Surprisingly, $\left(\mathbb{F}, \mathbb{F}^{+}\right)$is amenable, where $\mathbb{F}$ is a free group on a set of generators $S$ and $\mathbb{F}$ is the unital semigroup generated by $S$. This happens because $\mathrm{C}^{*}\left(\mathbb{F}, \mathbb{F}^{+}\right)$is considerably smaller than the Toeplitz algebra associated to the trivial product system $\left(\mathbb{C} u_{p}\right)_{p \in \mathbb{F}^{+}}$. For example, $\mathrm{C}^{*}\left(\mathbb{F}, \mathbb{F}^{+}\right)$ is spanned by elements of the form $v_{p} v_{q}^{*}$ with $p, q$ in $\mathbb{F}^{+}$.

The work of Nica described above together with the work of Laca and Raeburn [35] led Fowler to consider a class of product systems over positive cones of quasi-lattice ordered groups, called compactly aligned. He defined a notion of Nica covariance for representations of such product systems. The universal $\mathrm{C}^{*}$-algebra for this class of representations is then called Nica-Toeplitz algebra and denoted by $\mathcal{N} \mathcal{T}_{\mathcal{E}}$. For amenable systems, Fowler was able to characterise faithful representations of this algebra [26, Theorem 7.2]. Also under an amenability assumption, a result deriving nuclearity for a Nica-Toeplitz algebra from nuclearity of the underlying coefficient algebra was established in 53, Theorem 6.3].

However, the problem of finding a $\mathrm{C}^{*}$-algebra that approximates the structure of a given compactly aligned product system in an optimal way has not been completely solved. The question is: for a compactly aligned product system $\mathcal{E}=\left(\mathcal{E}_{p}\right)_{p \in P}$, which quotient of the Nica-Toeplitz algebra $\mathcal{N} \mathcal{T}_{\mathcal{E}}$ gives in an appropriate sense the smallest $\mathrm{C}^{*}$-algebra so that the representation of $\mathcal{E}$ in the corresponding quotient remains injective? Such a $\mathrm{C}^{*}$-algebra would be a co-universal object for Nica covariant representations satisfying a certain compatibility condition, at least under an amenability assumption.

${ }^{1} \mathrm{~A} \mathrm{C}^{*}$-algebra $A$ is nuclear if for every $\mathrm{C}^{*}$-algebra $B$ there exists a unique $\mathrm{C}^{*}$-norm on the tensor product $A \odot B$. 
Answering the above question was the main objective of the work of Sims and Yeend in [55]. They were able to associate a $\mathrm{C}^{*}$-algebra $\mathcal{N O}_{\mathcal{E}}$ to a given compactly aligned product system $\mathcal{E}$, called Cuntz-Nica-Pimsner algebra, so that the universal representation of $\mathcal{E}$ in $\mathcal{N} \mathcal{O}_{\mathcal{E}}$ is injective for a large class of product systems $[55$, Theorem 4.1]. This is a quotient of the Nica-Toeplitz algebra of $\mathcal{E}$. Their notion of covariant representations is more technical than the usual Cuntz-Pimsner covariance since it involves additional relations. Sims and Yeend proved that Cuntz-Nica-Pimsner algebras include Cuntz-Krieger algebras of finitely aligned higher-rank graphs [55, Proposition 5.4] and Katsura's relative Cuntz-Pimsner algebras of single correspondences [55, Proposition 5.3]. The analysis of co-universal properties for these algebras was provided in 15. If either $\mathcal{E}$ is faithful or the representation of $\mathcal{E}$ in $\mathcal{N} \mathcal{O}_{\mathcal{E}}$ is injective and $P$ is directed, then under an amenability assumption $\mathcal{N} \mathcal{O}_{\mathcal{E}}$ satisfies a gauge-invariant uniqueness property. That is, in the appropriate setting a representation of $\mathcal{N} \mathcal{O}_{\mathcal{E}}$ is faithful if and only if it is faithful on $A$ [15, Corollary 4.11].

Even though the universal representation of a compactly aligned product system $\mathcal{E}$ in $\mathcal{N} \mathcal{O}_{\mathcal{E}}$ is injective for many examples, it might fail to be faithful even for proper product systems over totally ordered semigroups such as the positive cone of $\mathbb{Z} \times \mathbb{Z}$ with the lexicographic order 55 , Example 3.16]. In addition, 15. Example 3.9] shows that if $P$ is not directed ${ }^{2}$ a representation of $\mathcal{N O} \mathcal{E}_{\mathcal{E}}$ that is faithful on $A$ need not be faithful even for an amenable system. We address these problems in Chapter 6, we attach a $\mathrm{C}^{*}$-algebra $A \times{ }_{\mathcal{E}} P$ to $\mathcal{E}$ so that $A$ embeds into $A \times{ }_{\mathcal{E}} P$ and given a surjective *-homomorphism $\psi: \mathcal{N} \mathcal{T}_{\mathcal{E}} \rightarrow B$ that is injective on $A$, under the standard assumptions of compatibility and amenability there exists a unique ${ }^{*}$-homomorphism $\phi: B \rightarrow A \times_{\mathcal{E}} P$ making the diagram

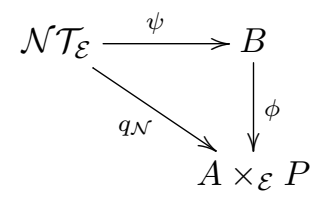

commute, where $q_{\mathcal{N}}$ is the quotient map. The compatibility assumption will be explained in more details in the subsequent section.

\subsection{Fell bundles over quasi-lattice ordered groups}

If a $\mathrm{C}^{*}$-algebra $B$ carries a continuous action of the unit circle $\mathbb{T}$, then many properties of $B$ can be derived from those of a certain $\mathrm{C}^{*}$-subalgebra, known as the fixed-point algebra for the $\mathbb{T}$-action. The latter might be much smaller than $B$ but, for instance, injectivity of representations of $B$ in many cases may be deduced from injectivity on this fixed-point algebra.

Let us illustrate this by an example. Let $\mathcal{T}$ denote the Toeplitz algebra. This is the Banach subalgebra of $\mathbb{B}\left(\ell_{2}(\mathbb{N})\right)$ generated by the unilateral shift $S$ on $\ell^{2}(\mathbb{N})$ and its adjoint $S^{*}$. Then $\mathbb{T}$ acts continuously on $\mathcal{T}$ by

$$
\mathbb{T} \ni z \mapsto \operatorname{Ad}_{U_{z}} \in \operatorname{Aut}(\mathcal{T})
$$

where $U_{z}$ is the unitary in $\mathbb{B}\left(\ell_{2}(\mathbb{N})\right)$ which sends a unit vector $e_{i}$ to $z^{i} e_{i}$ for all $i \in \mathbb{N}$ and $\operatorname{Ad}_{U_{z}}(a)=$ $U_{z} a U_{z}^{*}$. The closed subspace of $\mathcal{T}$ generated by the set $\left\{S^{i}\left(S^{*}\right)^{j} \mid i-j=n\right\}$ coincides with

$$
\mathcal{T}_{n}:=\left\{a \in \mathcal{T} \mid \operatorname{Ad}_{U_{z}}(a)=z^{n} a, \forall z \in \mathbb{T}\right\}
$$

These subspaces satisfy $\mathcal{T}_{m} \cdot \mathcal{T}_{n} \subseteq \mathcal{T}_{m+n}$ and $\mathcal{T}_{n}^{*}=\mathcal{T}_{-n}$ for all $m, n$ in $\mathbb{Z}$. There is a contractive projection of $\mathcal{T}$ onto $\mathcal{T}_{n}$ that vanishes on $\mathcal{T}_{m}$ for $m \neq n$, given by $a \mapsto \int_{\mathbb{T}} z^{-n} \operatorname{Ad}_{U_{z}}(a) \mathrm{d} z$. In addition, $\mathcal{T}$ is the closure of the direct sum $\bigoplus_{n \in \mathbb{Z}} \mathcal{T}_{n}$. Any representation of $\mathcal{T}$ that is injective on $\mathcal{T}_{0}$ sends $S$ to a proper isometry, and hence is injective on $\mathcal{T}$ by Coburn's Theorem.

The above facts also hold when one replaces $\mathbb{T}$ by any other compact abelian group and $\mathbb{Z}$ by the corresponding dual group. A $\mathrm{C}^{*}$-algebra $B$ acted upon by a compact abelian group $\Gamma$ may be described as the closure of a direct sum of closed subspaces indexed by the dual group $\widehat{\Gamma}$. There are mutually orthogonal contractive projections $\left\{E_{g}\right\}_{g \in \widehat{\Gamma}}$, where $E_{g}$ is the contractive projection of $B$ onto the corresponding subspace at $g$ for each $g$ in $\widehat{\Gamma}$, and the subspace at the unit element $e$ of $\widehat{\Gamma}$ is a $\mathrm{C}^{*}$-subalgebra of $B$. This provides $B$ with an extra structure, so that many important properties of

\footnotetext{
${ }^{2}$ We say that $P$ is directed if $p \vee q$ exists for all $p, q \in P$.
} 


\section{INTRODUCTION}

the associated fixed-point algebra are related to those of $B$.

What happens for non-abelian groups? A discrete group $G$ always carries a comultiplication. This is the ${ }^{*}$-homomorphism $\delta_{G}: \mathrm{C}^{*}(G) \rightarrow \mathrm{C}^{*}(G) \otimes \mathrm{C}^{*}(G)$ obtained from the unitary representation of $G$ in $\mathrm{C}^{*}(G) \otimes \mathrm{C}^{*}(G)$ which sends $g$ to $u_{g} \otimes u_{g}$, where $u_{g}$ denotes the image of $g$ under the canonical representation of $G$ in $\mathrm{C}^{*}(G)$. A full coaction of $G$ on a $\mathrm{C}^{*}$-algebra $B$ is an injective and nondegenerate *homomorphism $\delta: B \rightarrow B \otimes \mathrm{C}^{*}(G)$ satisfying the identity $\left(\delta \otimes \operatorname{id}_{\mathrm{C}^{*}(G)}\right) \circ \delta=\left(\operatorname{id}_{B} \otimes \delta_{G}\right) \circ \delta$. Nondegeneracy means that $B \otimes \mathrm{C}^{*}(G)$ is the closure of $\delta(B)\left(B \otimes \mathrm{C}^{*}(G)\right)$. Here the term "full" is related to the appearance of the full group $\mathrm{C}^{*}$-algebra of $G$ instead of its reduced $\mathrm{C}^{*}$-algebra $\mathrm{C}_{r}^{*}(G)$. If a $\mathrm{C}^{*}$-algebra $B$ comes equipped with a coaction of a discrete group $G$, then $B$ is the closure of the direct sum $\bigoplus_{g \in G} B_{g}$, where $B_{g}=\left\{a \in B \mid \delta(a)=a \otimes u_{g}\right\}$. As above, $B_{g} \cdot B_{h} \subseteq B_{g h}$ and $B_{g}^{*}=B_{g-1}$ for all $g, h \in G$, and there are contractive projections $E_{g}: B \rightarrow B_{g}$, so that $E_{g} \equiv 0$ on $B_{h}$ if $h \neq g$. In this case, we say that $B$ is a topologically $G$-graded $\mathrm{C}^{*}$-algebra and the collection of subspaces $\left\{B_{g}\right\}_{g \in G}$ is a topological grading for $B$.

Unfortunately there might be many non-isomorphic $\mathrm{C}^{*}$-algebras with indistinguishable topological $G$-grading. An important question to answer concerning a $G$-grading $\left\{B_{g}\right\}_{g \in G}$ is whether or not all of the $\mathrm{C}^{*}$-algebras possessing $\left\{B_{g}\right\}_{g \in G}$ as a topological $G$-grading are isomorphic to each other in the natural way. If the answer for this question is affirmative, in several cases many properties of the larger $\mathrm{C}^{*}$-algebra may be derived from those of the $\mathrm{C}^{*}$-algebra $B_{e}$. This is related to an amenability condition, which we will explain below.

Loosely speaking, a family of Banach spaces $\left(B_{g}\right)_{g \in G}$ endowed with a multiplication

$$
\cdot\left(B_{g}\right)_{g \in G} \times\left(B_{g}\right)_{g \in G} \rightarrow\left(B_{g}\right)_{g \in G}
$$

and an involution operation $*:\left(B_{g}\right)_{g \in G} \rightarrow\left(B_{g}\right)_{g \in G}$ is a Fell bundle, also known as a $\mathrm{C}^{*}$-algebraic bundle, if the triple $\left(\left(B_{g}\right)_{g \in G}, \cdot, *\right)$ behaves as a $G$-grading for a $\mathrm{C}^{*}$-algebra. So, for example, it must satisfy $B_{g} \cdot B_{h} \subseteq B_{g h}, B_{g}^{*}=B_{g^{-1}}$ and $\left\|b^{*} b\right\|=\|b\|^{2}$ for all $g, h \in G$ and $b \in B_{g}$.

One can naturally attach two $\mathrm{C}^{*}$-algebras to a given Fell bundle $\left(B_{g}\right)_{g \in G}$. Its cross sectional $\mathrm{C}^{*}$-algebra $\mathrm{C}^{*}\left(\left(B_{g}\right)_{g \in G}\right)$ is defined to be the universal $\mathrm{C}^{*}$-algebra for representations of $\left(B_{g}\right)_{g \in G}$. The reduced cross sectional $\mathrm{C}^{*}$-algebra $\mathrm{C}_{r}^{*}\left(\left(B_{g}\right)_{g \in G}\right)$ is constructed concretely through the regular representation of $\left(B_{g}\right)_{g \in G}$. Both $\mathrm{C}^{*}\left(\left(B_{g}\right)_{g \in G}\right)$ and $\mathrm{C}_{r}^{*}\left(\left(B_{g}\right)_{g \in G}\right)$ are topologically $G$-graded $\mathrm{C}^{*}$-algebras with grading given by a copy of $\left(B_{g}\right)_{g \in G}$. The latter has a co-universal property because it is the smallest $\mathrm{C}^{*}$-algebra whose topological $G$-grading is determined by a copy of the Fell bundle $\left(B_{g}\right)_{g \in G}$. We then say that $\left(B_{g}\right)_{g \in G}$ is amenable if its regular representation induces a ${ }^{*}$-isomorphism $\mathrm{C}^{*}\left(\left(B_{g}\right)_{g \in G} \cong\right.$ $\mathrm{C}_{r}^{*}\left(\left(B_{g}\right)_{g \in G}\right)$. So, up to canonical isomorphism, there is a unique topologically $G$-graded $\mathrm{C}^{*}$-algebra whose grading is a copy of $\left(B_{g}\right)_{g \in G}$. This is always so if $G$ is an amenable group.

If $g \mapsto \alpha_{g}$ is an action of $G$ by *-automorphisms on a $\mathrm{C}^{*}$-algebra $A$, one can build a Fell bundle out of the dynamical system $(A, G, \alpha)$ by letting $B_{g}:=(A, g)$ for each $g \in G$, that is, $B_{g}$ is a copy of $A$ as a Banach space. The multiplication on $\left(B_{g}\right)_{g \in G}$ is defined by convolution: $(a, g) *(b, h)=\left(a \alpha_{g}(b), g h\right)$, for $a, b$ in $A$ and $g, h$ in $G$. The involution operation $(A, g) \rightarrow\left(A, g^{-1}\right)$ sends $(a, g)$ to $\left(\alpha_{g^{-1}}\left(a^{*}\right), g^{-1}\right)$. The crossed product $A \rtimes_{\alpha} G$ coincides with the cross sectional $\mathrm{C}^{*}$-algebra of $\left(B_{g}\right)_{g \in G}$, while the reduced crossed product $A \rtimes_{\alpha, r} G$ is the reduced cross sectional $\mathrm{C}^{*}$-algebra $\mathrm{C}_{r}^{*}\left(\left(B_{g}\right)_{g \in G}\right)$. With some extra effort, one can also associate a Fell bundle to a twisted partial action. Hence cross sectional $\mathrm{C}^{*}$-algebras of Fell bundles include many $\mathrm{C}^{*}$-constructions built out of reversible dynamical systems. In particular, Fell bundles may also be viewed as generalised group actions.

Given a Fell bundle $\left(B_{g}\right)_{g \in G}$, each $B_{g}$ is a Hilbert $B_{e}$-module with right $B_{e^{-m o d u l e ~ s t r u c t u r e ~}}$ determined by the multiplication on $\left(B_{g}\right)_{g \in G}$ and inner product $\langle\xi \mid \eta\rangle:=\xi^{*} \eta \in B_{e}$, where "** is the involution operation on $\left(B_{g}\right)_{g \in G}$. In fact, the Hilbert $B_{e}$-module $B_{g}$ has more structure. It is also a Hilbert $B_{e}$-bimodule with left $B_{e}$-module action implemented by multiplication on the left by elements of $B_{e}$ and left inner product $\langle\langle\xi \mid \eta\rangle\rangle:=\xi \eta^{*}$. So it corresponds to a partial action of $G$ by Hilbert bimodules over $B_{e}$.

Relative Cuntz-Pimsner algebras of single correspondences come with a continuous $\mathbb{T}$-action, for which the image of $A$ under the universal representation lies in the fixed-point algebra. In $[29], \mathcal{O}_{J_{\mathcal{E}}, \mathcal{E}}$ is shown to satisfy a gauge-invariant uniqueness theorem. That is, any representation of $\mathcal{E}$ covariant on $J_{\mathcal{E}}$ that is faithful on $A$ and respects the topological $\mathbb{Z}$-grading associated to the $\mathbb{T}$-action is also faithful on $\mathcal{O}_{J_{\mathcal{E}}, \mathcal{E}}[29$, Theorem 6.4]. In general, the canonical $\mathbb{Z}$-grading of a relative Cuntz-Pimsner algebra is always a semi-saturated Fell bundle, in the sense that $B_{m} B_{n}=B_{m+n}$ if $m, n \geq 0$ [1]. The cross sectional $\mathrm{C}^{*}$-algebra of a semi-saturated Fell bundle over $\mathbb{Z}$ coincides with the crossed product 
of $B_{0}$ by the Hilbert bimodule $B_{1}$, which in turn is a relative Cuntz-Pimsner algebra as considered by Katsura. In particular, a semi-saturated Fell bundle over $\mathbb{Z}$ is generated by $B_{0}$ and the Hilbert bimodule $B_{1}$. So, up to isomorphism, semi-saturated partial actions of $\mathbb{Z}$ by Hilbert bimodules over $B_{0}$ are in bijection with actions of $\mathbb{N}$ by Hilbert $B_{0}$-bimodules.

Thus relative Cuntz-Pimsner algebras are completely determined by the spectral subspaces $\mathcal{O}_{J, \mathcal{E}}^{0}$ and $\mathcal{O}_{J, \mathcal{E}}^{1}$. In fact, the idea behind Pimsner's original $\mathrm{C}^{*}$-construction was to approximate a correspondence $\mathcal{E}: A \leadsto A$ by a Hilbert bimodule $\mathcal{O}_{\mathcal{E}}^{1}: \mathcal{O}_{\mathcal{E}}^{0} \leadsto \mathcal{O}_{\mathcal{E}}^{0}$ in the optimal way. From $\left(\mathcal{O}_{\mathcal{E}}^{0}, \mathcal{O}_{\mathcal{E}}^{1}\right)$ one can explicitly build a Fell bundle $\left(\mathcal{O}_{\mathcal{E}}^{n}\right)_{n \in \mathbb{Z}}$ by letting

$$
\mathcal{O}_{\mathcal{E}}^{n}= \begin{cases}\left(\mathcal{O}_{\mathcal{E}}^{1}\right)^{\otimes n} & \text { if } n>0 \\ \left(\left(\mathcal{O}_{\mathcal{E}}^{1}\right)^{*}\right)^{\otimes-n} & \text { if } n<0\end{cases}
$$

where $\left(\mathcal{O}_{\mathcal{E}}^{1}\right)^{*}$ is the Hilbert $\mathcal{O}_{\mathcal{E}}^{0}$-bimodule adjoint to $\mathcal{O}_{\mathcal{E}}^{1}$. The Cuntz-Pimsner algebra $\mathcal{O}_{\mathcal{E}}$ is the cross sectional $\mathrm{C}^{*}$-algebra of $\left(\mathcal{O}_{\mathcal{E}}^{n}\right)_{n \in \mathbb{Z}}$. Katsura's relative Cuntz-Pimsner algebra has a similar description.

For a quasi-lattice ordered group $(G, P)$, the Nica-Toeplitz algebra of a compactly aligned product system $\mathcal{E}=\left(\mathcal{E}_{p}\right)_{p \in P}$ carries a full coaction of $G$, obtained from a canonical Nica covariant representation of $\mathcal{E}$ in $\mathcal{N} \mathcal{T}_{\mathcal{E}} \otimes \mathrm{C}^{*}(G)$. This provides $\mathcal{N} \mathcal{T}_{\mathcal{E}}$ with a topological $G$-grading $\left\{\mathcal{N} \mathcal{T}_{\mathcal{E}}^{g}\right\}_{g \in G}$, for which the restriction to the positive fibres gives a product system $\left(\mathcal{N} \mathcal{T}_{\mathcal{E}}^{p}\right)_{p \in P}$. Such a product system has a special feature, which we prove in Chapter 5. First, it is a compactly aligned product system of Hilbert bimodules over $\mathcal{N} \mathcal{T}_{\mathcal{E}}^{e}$. Secondly, it can be explicitly extended to a Fell bundle over $G$ or, in other words, to a partial action of $G$ by Hilbert bimodules over $\mathcal{N} \mathcal{T}_{\mathcal{E}}^{e}$. The resulting Fell bundle is canonically isomorphic to $\left(\mathcal{N} \mathcal{T}_{\mathcal{E}}^{g}\right)_{g \in G}$. These assertions follow from a stronger result, which we will describe now.

We follow the terminology of [19] and define notions of orthogonality and semi-saturatedness for a Fell bundle over $G$. In one of our main results in Chapter 5 namely Theorem 5.1.8, we provide sufficient conditions for a compactly aligned product system of Hilbert bimodules over $P$ to extend to a semi-saturated and orthogonal Fell bundle over $G$. We say that a product system of Hilbert bimodules satisfying such conditions is simplifiable. The theorem in question has the following statement:

Theorem. Let $(G, P)$ be a quasi-lattice ordered group and let $\mathcal{E}=\left(\mathcal{E}_{p}\right)_{p \in P}$ be a simplifiable product system of Hilbert bimodules. There is a semi-saturated and orthogonal Fell bundle $\hat{\mathcal{E}}=\left(\hat{\mathcal{E}}_{g}\right)_{g \in G}$ extending the structure of product system of $\mathcal{E}$, in the sense that

(i) there are isomorphisms $j_{p}: \mathcal{E}_{p} \cong \hat{\mathcal{E}}_{p}$ of complex vector spaces such that $j_{e}: A \rightarrow \hat{\mathcal{E}}_{e}$ is a ${ }^{*}$-isomorphism and $j_{p}(\xi) j_{q}(\eta)=j_{p q}\left(\mu_{p, q}(\xi \otimes \eta)\right)$ for all $p, q \in P$;

(ii) $j_{p}(\xi)^{*} j_{p}(\eta)=j_{e}(\langle\xi \mid \eta\rangle)$ for all $\xi, \eta \in \mathcal{E}_{p}$ and $p \in P$, where ${ }^{*}: \hat{\mathcal{E}}_{p} \rightarrow \hat{\mathcal{E}}_{p^{-1}}$ is the involution operation on $\hat{\mathcal{E}}$.

Moreover, $\hat{\mathcal{E}}$ is unique up to canonical isomorphism of Fell bundles.

As a consequence, the universal representation of a simplifiable product system of Hilbert bimodules in the relative Cuntz-Pimsner algebra for the family of Katsura's ideals is always injective. This implies the following corollary:

Corollary. Let $\mathcal{E}=\left(\mathcal{E}_{p}\right)_{p \in P}$ be a simplifiable product system of Hilbert bimodules over $A$. For each $p \in P$, set $I_{p}:=\left\langle\left\langle\mathcal{E}_{p} \mid \mathcal{E}_{p}\right\rangle\right\rangle$ and $\mathcal{I}=\left\{I_{p}\right\}_{p \in P}$. Then the canonical ${ }^{*}$-homomorphism from $A$ to the relative Cuntz-Pimsner algebra $\mathcal{O}_{\mathcal{I}, \mathcal{E}}$ is an isomorphism onto the gauge-fixed point algebra $\mathcal{O}_{\mathcal{I}, \mathcal{E}}^{e}$. Moreover, $\mathcal{O}_{\mathcal{I}, \mathcal{E}}^{p} \cong \mathcal{E}_{p}$ for all $p \in P$.

In addition, we show that a Fell bundle that is semi-saturated and orthogonal comes from a simplifiable product system of Hilbert bimodules. This gives an equivalence between a class of partial actions of $G$ by Hilbert bimodules and simplifiable actions of $P$. So we say that a semi-saturated and orthogonal Fell bundle over $G$ is extended from $P$. The cross sectional $\mathrm{C}^{*}$-algebra of a Fell bundle extended from $P$ can be described as a relative Cuntz-Pimsner algebra of a simplifiable product system of Hilbert bimodules. For a Fell bundle over $\mathbb{Z}$, orthogonality is vacuous and hence Fell bundles extended from $\mathbb{N}$ are precisely semi-saturated Fell bundles as described above.

Proposition. Let $\left(B_{g}\right)_{g \in G}$ be a Fell bundle that is semi-saturated and orthogonal with respect to $(G, P)$. Then $\mathcal{B}=\left(B_{p}\right)_{p \in P}$ is a simplifiable product system of Hilbert bimodules. Its relative Cuntz-Pimsner algebra $\mathcal{O}_{\mathcal{I}, \mathcal{B}}$ is naturally isomorphic to the cross sectional $\mathrm{C}^{*}$-algebra of $\left(B_{g}\right)_{g \in G}$. 


\section{INTRODUCTION}

In Section 5.3, we describe the cross sectional $\mathrm{C}^{*}$-algebra of a Fell bundle extended from $\mathbb{F}^{+}$as a relative Cuntz-Pimsner of a single correspondence. This is used to establish amenability for such Fell bundles. This fact is established in [19] under a separability assumption. Our approach does not require such a hypothesis. We prove a similar result for Fell bundles extended from Baumslag-Solitar semigroups $\mathrm{BS}(c, d)^{+}$with $c, d$ positive integers.

\subsection{Bicategories of correspondences}

In a bicategory, we have a category $\mathcal{C}(x, y)$ of arrows between two objects $x$ and $y$ instead of a set of arrows. Associativity only holds up to isomorphisms of arrows and an object $x$ has a unit arrow only up to isomorphism. An arrow $f: x \rightarrow y$ is an equivalence, or is invertible, if there exists an arrow $g: y \rightarrow x$ with isomorphisms $g \circ f \cong 1_{x}$ and $f \circ g \cong 1_{y}$.

$\mathrm{C}^{*}$-algebras are the objects of the correspondence bicategory $\mathfrak{C}$ introduced by Buss, Meyer and $\mathrm{Zhu}$ in [14]. Arrows are correspondences between $\mathrm{C}^{*}$-algebras and 2-arrows are isomorphisms of correspondences. The composition of arrows is the internal tensor product of correspondences. Equivalences in $\mathfrak{C}$ correspond to Morita equivalences between $\mathrm{C}^{*}$-algebras. So a Hilbert bimodule $\mathcal{E}$ over a $\mathrm{C}^{*}$-algebra $A$ is an equivalence in $\mathfrak{C}$ from $\langle\langle\mathcal{E} \mid \mathcal{E}\rangle\rangle$ to $\langle\mathcal{E} \mid \mathcal{E}\rangle$. Since these are ideals in $A, \mathcal{E}$ may be interpreted as a partial Morita equivalence of $A$. A saturated Fell bundle over a discrete group $G$ is then equivalent to a homomorphism from $G$ to $\mathfrak{C}$ [14, Theorem 3.3]. Saturatedness means that $B_{g} \cdot B_{h}=B_{g h}$ for all $g, h$ in $G$. This supports the idea that Fell bundles are generalised group actions.

Assuming that $\mathcal{E}$ is a proper correspondence, that is, $\varphi(A) \subseteq \mathbb{K}(\mathcal{E})$, Albandik and Meyer provided in 6] a description of $\mathcal{O}_{\mathcal{E}}=\mathcal{O}_{A, \mathcal{E}}$ as a colimit of a diagram in the correspondence bicategory. This is generalised in Chapter 3 (see also [40]) to relative Cuntz-Pimsner algebras of (not necessarily proper) correspondences by looking at another bicategory of correspondences, obtained from the bicategory of homomorphisms, or "functors", from $\mathbb{N}$ to $\mathfrak{C}$ after some modifications. More explicitly, we define a bicategory $\mathfrak{C}_{\mathrm{pr}}^{\mathbb{N}}$ whose objects are triples $(A, \mathcal{E}, J)$, where $A$ is a $\mathrm{C}^{*}$-algebra, $\mathcal{E}$ is a correspondence over $A$, and $J$ is an ideal in $A$ that acts by compact operators on $\mathcal{E}$. This is precisely the data needed to define a relative Cuntz-Pimsner algebra. An arrow $(\mathcal{F}, V):(A, \mathcal{E}, J) \rightarrow\left(A_{1}, \mathcal{E}_{1}, J_{1}\right)$ consists of a proper correspondence $\mathcal{F}: A \leadsto A_{1}$ with $J \cdot \mathcal{F} \subseteq \mathcal{F} \cdot J_{1}$ and a correspondence isomorphism $V: \mathcal{E} \otimes_{A} \mathcal{F} \cong \mathcal{F} \otimes_{A_{1}} \mathcal{E}_{1}$. The pair $(\mathcal{F}, V)$ is called a proper covariant correspondence.

The triples $\left(A, \mathcal{E}, I_{\mathcal{E}}\right)$ where $\mathcal{E}$ is a Hilbert $A$ bimodule and $I_{\mathcal{E}}$ is Katsura's ideal for $\mathcal{E}$ form a full sub-bicategory of $\mathfrak{C}_{\mathrm{pr}}^{\mathbb{N}}$. which we denote by $\mathfrak{C}_{\mathrm{pr}, *}^{\mathbb{N}}$. We establish the existence of a universal arrow

$$
v_{(A, \mathcal{E}, J)}:(A, \mathcal{E}, J) \rightarrow\left(\mathcal{O}_{J, \mathcal{E}}^{0}, \mathcal{O}_{J, \mathcal{E}}^{1}, I_{\mathcal{O}_{J, \mathcal{E}}^{1}}\right)
$$

from $(A, \mathcal{E}, J)$ to the inclusion $\mathfrak{C}_{\mathrm{pr}, *}^{\mathbb{N}} \hookrightarrow \mathfrak{C}_{\mathrm{pr}}^{\mathbb{N}}$ in Proposition 3.3 .3 Combining this with some general results from bicategory theory, we show in Corollary 3.3 .7 that the pair $\left(\mathcal{O}_{J, \mathcal{E}}^{0}, \mathcal{O}_{J, \mathcal{E}}^{1}\right)$ describes part of a reflector from $\mathfrak{C}_{\mathrm{pr}}^{\mathbb{N}}$ onto $\mathfrak{C}_{\mathrm{pr}, *}^{\mathbb{N}}$. Roughly speaking, a reflector approximates a given object by an object in the sub-bicategory in the optimal way. It is a left (bi)adjoint to the inclusion of the sub-bicategory. So $\mathcal{O}_{J, \mathcal{E}}^{1}: \mathcal{O}_{J, \mathcal{E}}^{0} \leadsto \mathcal{O}_{J, \mathcal{E}}^{0}$ is indeed the best approximation of $\mathcal{E}: A \leadsto A$ by a Hilbert bimodule, satisfying a certain covariance condition relying on the ideal $J$.

Given a quasi-lattice ordered group $(G, P)$, we define a bicategory $\mathfrak{C}^{P}$ in which objects are triples $(A, \mathcal{E}, \mathcal{J})$, where $A$ is a $\mathrm{C}^{*}$-algebra, $\mathcal{E}$ is a compactly aligned product system and $\mathcal{J}=\left\{J_{p}\right\}_{p \in P}$ is a family of ideals in $A$ with $J_{p} \subseteq \varphi_{p}^{-1}\left(\mathbb{K}\left(\mathcal{E}_{p}\right)\right)$ for all $p \in P$. We consider a sub-bicategory of $\mathfrak{C}^{P}$ whose objects are simplifiable product systems of Hilbert bimodules, which we denote by $\mathfrak{C}_{*}^{P}$. We enrich the correspondence between simplifiable product systems of Hilbert bimodules over $P$ and Fell bundles extended from $P$ to an equivalence between $\mathfrak{C}_{*}^{P}$ and a certain bicategory of Fell bundles extended from $P$. This equivalence is defined on objects by using the correspondence described in the previous section. We apply this equivalence to show that, when one restricts to proper covariant correspondences, the construction of relative Cuntz-Pimsner algebras as quotients of Nica-Toeplitz algebras gives a reflector from $\mathfrak{C}_{\mathrm{pr}}^{P}$ onto $\mathfrak{C}_{\mathrm{pr}, *}^{P}$. In particular, this construction is functorial. Hence equivalences in $\mathfrak{C}_{\mathrm{pr}}^{P}$ yield Morita equivalences between the corresponding relative Cuntz-Pimsner algebras.

A semigroup of extendible and injective endomorphisms with hereditary range produces a product system of Hilbert bimodules over P. We generalise the ideas of [42, Proposition 2.4] and characterise the invertible covariant correspondences between the associated product systems. This is achieved in 
Proposition 5.4 .9

\subsection{Covariance algebras of product systems}

The theory of relative Cuntz-Pimsner algebras of product systems does not cover constructions such as Cuntz-Krieger algebras of finitely aligned higher-rank graphs. The work of Exel in [19] suggests that his notion of crossed products by semigroups of unital endomorphisms cannot be covered by relative Cuntz-Pimsner algebras. As mentioned previously, if $P$ is a subsemigroup of a group $G$ so that $(G, P)$ is a quasi-lattice ordered group, Sims and Yeend introduced in $[55$. the so called Cuntz-Nica-Pimsner algebra $\mathcal{N} \mathcal{O}_{\mathcal{E}}$ associated to a compactly aligned product system $\mathcal{E}=\left(\mathcal{E}_{p}\right)_{p \in P}$. Their main goal was to associate a $\mathrm{C}^{*}$-algebra to a given compactly aligned product system satisfying two properties: the representation of $\mathcal{E}$ in $\mathcal{N O} \mathcal{O}_{\mathcal{E}}$ is injective on $A$, and a ${ }^{*}$-homomorphism from $\mathcal{N} \mathcal{O}_{\mathcal{E}}$ to a $\mathrm{C}^{*}$-algebra $B$ is faithful on the fixed-point algebra for the canonical coaction of $G$ on $\mathcal{N O} \mathcal{O}_{\mathcal{E}}$ if and only if it is faithful on $A$. These are the conditions (A) and (B) of [55], respectively. Although Sims and Yeend's $\mathrm{C}^{*}$-construction suffices for a large class of product systems, the universal representation of $\mathcal{E}$ in $\mathcal{N} \mathcal{O}_{\mathcal{E}}$ may fail to be injective. In addition, $\mathcal{N} \mathcal{O}_{\mathcal{E}}$ might not fulfil the condition (B) above even when it satisfies (A). It cannot handle product systems over semigroups that are not positive cones of quasi-lattice orders.

In Chapter 6, we let $P$ be a subsemigroup of a group $G$ and construct a $\mathrm{C}^{*}$-algebra from a product system $\mathcal{E}$ over $P$ satisfying the above conditions (A) and (B). To do so, we look at the topological $G$-grading $\left\{\mathcal{T}_{\mathcal{E}}^{g}\right\}_{g \in G}$ of the Toeplitz algebra of $\mathcal{E}$ coming from the canonical coaction of $G$. We analyse a class of representations of $\mathcal{T}_{\mathcal{E}}$ coming from quotients of the usual Fock representation of $\mathcal{E}$ on $\bigoplus_{p \in P} \mathcal{E}_{p}$. Constructions of [5, 19, 34, 48] led us to look for a $\mathrm{C}^{*}$-algebra whose spectral subspace at $g \in G$ is an inductive limit of quotients of $\mathcal{T}_{\mathcal{E}}^{g}$ in such a way that the connecting maps are all injective. So we consider the directed set consisting of finite subsets of $G$. After modifying the Cuntz-Nica-Pimsner covariance condition and adapting it to this more general setting, we arrive at what we call strong covariance. Although it explicitly involves elements of $G$, this notion of covariance does not depend on the embedding $P \hookrightarrow G$. In other words, different groups containing $P$ as a subsemigroup produce the same quotient of $\mathcal{T}_{\mathcal{E}}$. We refer to the universal $\mathrm{C}^{*}$-algebra for strongly covariant representations of $\mathcal{E}$ as its covariance algebra and denote it by $A \times_{\mathcal{E}} P$. We show that the universal representation of $\mathcal{E}$ in $A \times{ }_{\mathcal{E}} P$ is injective. In addition, $A \times{ }_{\mathcal{E}} P$ satisfies condition (B): a representation of $A \times{ }_{\mathcal{E}} P$ is faithful on the fixed-point algebra for the canonical coaction of $G$ if and only if it is faithful on the coefficient algebra $A$. More precisely, the next is our main theorem in Chapter 6 .

Theorem. Let $P$ be a unital semigroup and let $\mathcal{E}=\left(\mathcal{E}_{p}\right)_{p \in P}$ be a product system over $P$ of $A$-correspondences. Suppose that $P$ is embeddable into a group. There is a $\mathrm{C}^{*}$-algebra $A \times{ }_{\mathcal{E}} P$ associated to $\mathcal{E}$ with a representation $j_{\mathcal{E}}: \mathcal{E} \rightarrow A \times_{\mathcal{E}} P$ such that the pair $\left(A \times_{\mathcal{E}} P, j_{\mathcal{E}}\right)$ has the following properties:

(C1) $A \times_{\mathcal{E}} P$ is generated by $j_{\mathcal{E}}(\mathcal{E})$ as a $\mathrm{C}^{*}$-algebra and $j_{\mathcal{E}}$ is strongly covariant in the sense of Definition 6.1.2, where the group $G$ in question may be taken to be any group containing $P$ as a subsemigroup.

$(\mathrm{C} 2)$ if $\psi=\left\{\psi_{p}\right\}_{p \in P}$ is a strongly covariant representation of $\mathcal{E}$ in a $\mathrm{C}^{*}$-algebra $B$ with respect to a group containing $P$, then there is a unique ${ }^{*}$-homomorphism $\widehat{\psi}: A \times{ }_{\mathcal{E}} P \rightarrow B$ such that $\widehat{\psi} \circ j_{p}=\psi_{p}$ for all $p \in P$;

(C3) $j_{e}$ is faithful and if $G$ is a group with $P \subseteq G$ as a subsemigroup, there is a canonical full coaction of $G$ on $A \times{ }_{\mathcal{E}} P$ so that $a{ }^{*}$-homomorphism $A \times{ }_{\mathcal{E}} P \rightarrow B$ is faithful on the fixed-point algebra $\left(A \times_{\mathcal{E}} P\right)^{\delta}$ if and only if it is faithful on $j_{e}(A)$.

Up to canonical isomorphism, $\left(A \times_{\mathcal{E}} P, j_{\mathcal{E}}\right)$ is the unique pair with the properties $(\mathrm{C} 1)-(\mathrm{C} 3)$.

The notion of Cuntz-Pimsner covariance introduced here is technical and in general difficult to verify. However, this construction includes Fowler's (relative) Cuntz-Pimsner algebra if $\mathcal{E}$ is a proper and faithful product system over a cancellative Ore monoid. It also includes relative Cuntz-Pimsner algebras of simplifiable product systems of Hilbert bimodules for the family of Katsura's ideals if $(G, P)$ is a quasi-lattice ordered group. For a compactly aligned product system $\mathcal{E}$, we find an equivalent notion of covariant representations that is considerably simpler to verify. We show that $A \times{ }_{\mathcal{E}} P$ 


\section{INTRODUCTION}

coincides with $\mathcal{N} \mathcal{O}_{\mathcal{E}}$ if this latter $\mathrm{C}^{*}$-algebra also satisfies conditions $(\mathrm{A})$ and $(\mathrm{B})$. This happens when $\mathcal{E}$ is faithful or the representation of $\mathcal{E}$ in $\mathcal{N O} \mathcal{O}_{\mathcal{E}}$ is injective and $P$ is directed [15. Proposition 3.7].

Again only assuming that $P$ is embeddable into a group, we construct a product system $\mathcal{E}$ as in [5. Section 5] so that $A \times_{\mathcal{E}} P$ recovers the semigroup $\mathrm{C}^{*}$-algebra of Xin Li whenever the family of constructible right ideals of $P$ is independent (see [39, Definition 2.26]). In general, the covariance algebra of such a product system corresponds to the semigroup $\mathrm{C}^{*}$-algebra $\mathrm{C}_{s}^{*}(\cup)(P)$ in the notation of [39]. In the last subsection, we assume that $P$ is a reversible cancellative semigroup and describe a class of Exel's crossed products by interaction groups as covariance algebras. Thus our approach may inspire further $\mathrm{C}^{*}$-constructions for irreversible dynamical systems. 


\section{Chapter 2}

\section{Fell bundles and coactions}

This chapter introduces our main tools to study $\mathrm{C}^{*}$-algebras associated to product systems. We begin with the definition of Fell bundles over discrete groups and the constructions of their full and reduced cross sectional $\mathrm{C}^{*}$-algebras. In Section 2.2 we focus on $\mathrm{C}^{*}$-algebras equipped with a topological grading. This class of $\mathrm{C}^{*}$-algebras contains those algebras associated to Fell bundles. They arise naturally from coactions of discrete groups. We will see that the reduced $\mathrm{C}^{*}$-algebra of a Fell bundle has a certain co-universal property among topologically graded $\mathrm{C}^{*}$-algebras (see Theorem 2.2.9).

Our main references for this chapter are [18], 23] and [50]. We refer to [43 for the basic theory of $\mathrm{C}^{*}$-algebras, while some basic constructions concerning Hilbert modules can be found in Appendix A

\section{$2.1 \quad$ Fell bundles}

\subsubsection{Cross sectional $\mathrm{C}^{*}$-algebra}

Definition 2.1.1. A Fell bundle over a discrete group $G$ consists of a collection of Banach spaces $\left(B_{g}\right)_{g \in G}$ endowed with multiplication maps $\cdot: B_{g} \times B_{h} \rightarrow B_{g h}$ and, for each $g \in G$, an involution ${ }^{*}: B_{g} \rightarrow B_{g^{-1}}$ satisfying for all $b_{g} \in B_{g}, b_{h} \in B_{h}, b_{r} \in B_{r}$ and $g, h, r$ in $G$

(i) the multiplication maps are bilinear and associative, that is, $\left(b_{g} b_{h}\right) b_{r}=b_{g}\left(b_{h} b_{r}\right)$;

(ii) ${ }^{*}: B_{g} \rightarrow B_{g^{-1}}$ is conjugate-linear and isometric;

(iii) $b_{g}^{* *}=b_{g}$;

(iv) $\left(b_{g} b_{h}\right)^{*}=b_{h}^{*} b_{g}^{*}$;

(v) $\left\|b_{g} b_{h}\right\| \leq\left\|b_{g}\right\|\left\|b_{h}\right\|$;

(vi) $\left\|b_{g}^{*} b_{g}\right\|=\left\|b_{g}\right\|^{2}$;

(vii) for each $b_{g} \in B_{g}$, there exists $a \in B_{e}$ with $b_{g}^{*} b_{g}=a^{*} a$.

We say that $B_{g}$ is the fibre of $\left(B_{g}\right)_{g \in G}$ at $g$. Observe that axioms (i)-(vi) imply that $B_{e}$ is a $\mathrm{C}^{*}$-algebra. We will often refer to $B_{e}$ as the unit fibre of $\left(B_{g}\right)_{g \in G}$. In order to fix notation, by $B_{g} B_{h}$ we mean the closed linear span of

$$
\left\{b_{g} b_{h} \mid b_{g} \in B_{g}, b_{h} \in B_{h}\right\} .
$$

In particular, $B_{g} B_{g^{-1}}$ is a closed two-sided ideal in $B_{e}$ for all $g \in G$.

Example 2.1.2. Let $G$ be a discrete group. We may associate a Fell bundle $\left(B_{g}\right)_{g \in G}$ to $G$ in a canonical way: we set $B_{g}:=\mathbb{C} \times\{g\}$ with the structure of Banach space inherited from $\mathbb{C}$. We write $\lambda u_{g}$ for $(\lambda, g)$ so that $B_{g}=\mathbb{C} u_{g}$. The multiplication and involution operations on $\mathbb{C}$ provide $\left(\mathbb{C} u_{g}\right)_{g \in G}$ with a structure of Fell bundle.

Example 2.1.3. Let $A=M_{3}(\mathbb{C})$. Let $B_{-1}, B_{0}$ and $B_{1}$ be the subspaces

$$
\left(\begin{array}{lll}
0 & 0 & 0 \\
* & 0 & 0 \\
* & 0 & 0
\end{array}\right), \quad\left(\begin{array}{ccc}
* & 0 & 0 \\
0 & * & * \\
0 & * & *
\end{array}\right) \text { and }\left(\begin{array}{ccc}
0 & * & * \\
0 & 0 & 0 \\
0 & 0 & 0
\end{array}\right)
$$




\section{FELL BUNDLES AND COACTIONS}

of $A$, respectively. For $n \in \mathbb{Z} \backslash\{-1,0,1\}$ put $B_{n}=\{0\}$. Then $\left(B_{n}\right)_{n \in \mathbb{Z}}$ equipped with the usual multiplication and involution operations defined on matrices is a Fell bundle over $\mathbb{Z}$.

Lemma 2.1.4. Let $\left(B_{g}\right)_{g \in G}$ be a Fell bundle. If $\left(u_{\lambda}\right)_{\lambda \in \Lambda}$ is an approximate identity for $B_{e}$, then for all $g \in G$ and $b_{g} \in B_{g}$

$$
\lim _{\lambda} b_{g} u_{\lambda}=\lim _{\lambda} u_{\lambda} b_{g}=b_{g}
$$

In order to construct a $\mathrm{C}^{*}$-algebra out of a Fell bundle $\left(B_{g}\right)_{g \in G}$, consider the complex vector space

$$
C_{c}\left(\left(B_{g}\right)_{g \in G}\right)=\left\{\xi: G \rightarrow \bigcup_{g \in G} B_{g} \mid \xi(g) \in B_{g} \forall g \in G \text { and } \operatorname{supp}(\xi) \text { is finite }\right\} .
$$

In other words, $C_{c}\left(\left(B_{g}\right)_{g \in G}\right)$ is the direct sum $\bigoplus_{g \in G} B_{g}$.

We make $C_{c}\left(\left(B_{g}\right)_{g \in G}\right)$ into a ${ }^{*}$-algebra as follows. We let the multiplication

$$
\left.*: C_{c}\left(\left(B_{g}\right)_{g \in G}\right)\right) \times C_{c}\left(\left(B_{g}\right)_{g \in G}\right) \rightarrow C_{c}\left(\left(B_{g}\right)_{g \in G}\right)
$$

be given by $(\xi, \eta) \mapsto \xi * \eta$, where

$$
(\xi * \eta)(h)=\sum_{g \in G} \xi(g) \eta\left(g^{-1} h\right)
$$

for all $h \in G$. The involution is defined by

$$
{ }^{*}: \xi \in C_{c}\left(\left(B_{g}\right)_{g \in G}\right) \mapsto \xi^{*} \in C_{c}\left(\left(B_{g}\right)_{g \in G}\right),
$$

where $\xi^{*}(g)=\xi\left(g^{-1}\right)^{*}$ for all $g \in G$. These operations provide $C_{c}\left(\left(B_{g}\right)_{g \in G}\right)$ with a structure of *-algebra.

For each $g \in G$, we may view $B_{g}$ as a subspace of $C_{c}\left(\left(B_{g}\right)_{g \in G}\right)$ through the identification $j_{g}: B_{g} \rightarrow C_{c}\left(\left(B_{g}\right)_{g \in G}\right)$,

$$
j_{g}\left(b_{g}\right)(h)= \begin{cases}b_{g} & \text { if } h=g \\ 0 & \text { otherwise }\end{cases}
$$

We will use this identification in the sequel.

Proposition 2.1.5. Let $\pi: C_{c}\left(\left(B_{g}\right)_{g \in G}\right) \rightarrow B$ be a representation of $C_{c}\left(\left(B_{g}\right)_{g \in G}\right)$ in a $\mathrm{C}^{*}$-algebra $B$ and let $\xi \in C_{c}\left(\left(B_{g}\right)_{g \in G}\right)$. Then $\|\pi(\xi)\| \leq\|\xi\|_{1}$, where $\|\xi\|_{1}=\sum_{g \in G}\|\xi(g)\|$.

Proof. The composite $\pi \circ j_{e}$ yields a ${ }^{*}$-homomorphism $B_{e} \rightarrow B$. Hence it is contractive, that is, $\left\|\pi\left(j_{e}(a)\right)\right\| \leq\|a\|$ for all $a \in A$. Given $c \in B_{g}$, we have

$$
\left\|\pi\left(j_{g}(c)\right)\right\|^{2}=\left\|\pi\left(j_{g}(c)\right)^{*} \pi\left(j_{g}(c)\right)\right\|=\left\|\pi\left(j_{g}(c)^{*} j_{g}(c)\right)\right\| \leq\left\|\pi\left(j_{e}\left(c^{*} c\right)\right)\right\|=\left\|c^{*} c\right\|=\|c\|^{2} .
$$

In general,

$$
\|\pi(\xi)\|=\left\|\sum_{g \in G} \pi\left(j_{g}(\xi(g))\right)\right\| \leq \sum_{g \in G}\|\xi(g)\|=\|\xi\|_{1} .
$$

Definition 2.1.6. Let $\left(B_{g}\right)_{g \in G}$ be a Fell bundle and $B$ a $C^{*}$-algebra. A representation of $\left(B_{g}\right)_{g \in G}$ in $B$ is a collection of linear maps $\pi=\left\{\pi_{g}\right\}_{g \in G}$, where $\pi_{g}: B_{g} \rightarrow B$, such that for all $b_{g} \in B_{g}, b_{h} \in B_{h}$ and $g, h \in G$ :

(i) $\pi_{g}\left(b_{g}\right) \pi_{h}\left(b_{h}\right)=\pi_{g h}\left(b_{g} b_{h}\right)$,

(ii) $\pi_{g}\left(b_{g}\right)^{*}=\pi_{g^{-1}}\left(b_{g}^{*}\right)$.

Proposition 2.1.5 implies $\rho(\xi) \leq\|\xi\|_{1}$ whenever $\rho$ is a $\mathrm{C}^{*}$-seminorm on $C_{c}\left(\left(B_{g}\right)_{g \in G}\right)$. So we let $\rho$ range in the collection of all $\mathrm{C}^{*}$-seminorms on $C_{c}\left(\left(B_{g}\right)_{g \in G}\right)$ and obtain a well defined $\mathrm{C}^{*}$-seminorm by setting

$$
\rho_{\infty}(\xi):=\sup _{\rho} \rho(\xi)
$$


Definition 2.1.7. The cross sectional $\mathrm{C}^{*}$-algebra of $\left(B_{g}\right)_{g \in G}$, denoted by $\mathrm{C}^{*}\left(\left(B_{g}\right)_{g \in G}\right)$, is the completion of $\left(C_{c}\left(\left(B_{g}\right)_{g \in G}\right), \rho_{\infty}\right)$.

Let $j=\left\{j_{g}\right\}_{g \in G}$, where $j_{g}: B_{g} \rightarrow C_{c}\left(\left(B_{g}\right)_{g \in G}\right)$ is the canonical inclusion. This gives rise to a representation of $\left(B_{g}\right)_{g \in G}$ in $\mathrm{C}^{*}\left(\left(B_{g}\right)_{g \in G}\right)$ obtained from the composition of $j$ with the canonical representation of $C_{c}\left(\left(B_{g}\right)_{g \in G}\right)$ in $\mathrm{C}^{*}\left(\left(B_{g}\right)_{g \in G}\right)$. We still denote this representation by $j=\left\{j_{g}\right\}_{g \in G}$. We will later see that $j_{g}$ is injective for all $g \in G$. For now we prove its universal property:

Proposition 2.1.8. Let $\pi=\left\{\pi_{g}\right\}_{g \in G}$ be a representation of $\left(B_{g}\right)_{g \in G}$ in a $\mathrm{C}^{*}$-algebra $B$. There is a unique*-homomorphism $\widehat{\pi}: \mathrm{C}^{*}\left(\left(B_{g}\right)_{g \in G}\right) \rightarrow B$ such that $\widehat{\pi} \circ j_{g}\left(b_{g}\right)=\pi_{g}\left(b_{g}\right)$ for all $g \in G$ and $b_{g} \in B_{g}$.

Proof. A representation of $\left(B_{g}\right)_{g \in G}$ produces a representation of $C_{c}\left(\left(B_{g}\right)_{g \in G}\right)$ by

$$
\xi \mapsto \sum_{g \in G} \pi_{g}(\xi(g))
$$

Such a representation extends uniquely to a ${ }^{*}$-homomorphism $\widehat{\pi}: \mathrm{C}^{*}\left(\left(B_{g}\right)_{g \in G}\right) \rightarrow B$ by universal property. This satisfies $\widehat{\pi} \circ j_{g}=\pi_{g}$ as asserted.

Example 2.1.9. If $G$ is a discrete group, the cross sectional $\mathrm{C}^{*}$-algebra of the trivial bundle $\left(\mathbb{C} u_{g}\right)_{g \in G}$ is the group $\mathrm{C}^{*}$-algebra $\mathrm{C}^{*}(G)$. More generally, given an action $G \rightarrow \operatorname{Aut}(A)$ on a $\mathrm{C}^{*}$-algebra $A$, one may build a Fell bundle out of $\alpha$ by setting $B_{g}:=A u_{g}$. That is, $B_{g}$ is a copy of $A$ as a Banach space. The multiplication is defined by

$$
\left(a u_{g}\right) \cdot\left(b u_{h}\right):=a \alpha_{g}(b) u_{g h},
$$

and the involution $A u_{g} \rightarrow A u_{g^{-1}}$ by

$$
\left(a u_{g}\right)^{*}:=\alpha_{g^{-1}}\left(a^{*}\right) u_{g^{-1}} .
$$

These operations turn $\left(A u_{g}\right)_{g \in G}$ into a Fell bundle over $G$. Its cross sectional $\mathrm{C}^{*}$-algebra is canonically isomorphic to the crossed product $A \rtimes_{\alpha} G$.

\subsubsection{The regular representation}

In this subsection, our main objective is to construct an injective representation of a given Fell bundle. We will use this representation to define its reduced cross sectional $\mathrm{C}^{*}$-algebra.

For each $g \in G$, view the Banach space $B_{g}$ as a right Hilbert $B_{e}$-module with right action and inner product inherited from $\left(B_{g}\right)_{g \in G}$. Explicitly, for $a \in B_{e}$ and $b, c \in B_{g}$, the right action is defined by $b \cdot a:=b a \in B_{g}$ and the inner product is given by $\langle b \mid c\rangle:=b^{*} c$. Consider the right Hilbert $B_{e}$-module $\ell_{2}\left(\left(B_{g}\right)_{g \in G}\right)$ built out of the direct sum of the $B_{g}$ 's. That is,

$$
\ell_{2}\left(\left(B_{g}\right)_{g \in G}\right)=\bigoplus_{g \in G} B_{g}
$$

This is a correspondence over $B_{e}$ with left action implemented by the diagonal operator associated to $a \in B_{e}$. In fact, we will construct a representation of $\left(B_{g}\right)_{g \in G}$ in the $\mathrm{C}^{*}$-algebra of adjointable operators on $\ell_{2}\left(\left(B_{g}\right)_{g \in G}\right)$. For each $g \in G$ and $b_{g} \in B_{g}$, let $\lambda_{g}\left(b_{g}\right)$ be defined on a finite sum $\xi=\sum \xi_{h}$ by

$$
\lambda_{g}\left(b_{g}\right)(\xi)_{h}:=b_{g} \xi_{g^{-1} h}
$$

This satisfies

$$
\left\langle\lambda_{g}\left(b_{g}\right) \xi \mid \lambda_{g}\left(b_{g}\right) \xi\right\rangle \leq\left\|b_{g}\right\|^{2}\langle\xi \mid \xi\rangle
$$

because

$$
\left\langle\xi_{h} \mid a^{*} a \xi_{h}\right\rangle \leq\|a\|^{2}\left\langle\xi_{h} \mid \xi_{h}\right\rangle
$$

for all $a \in B_{e}, \xi_{h} \in B_{h}$ and $h \in G$. Hence $\lambda_{g}\left(b_{g}\right)$ gives a well defined continuous operator on $\ell_{2}\left(\left(B_{g}\right)_{g \in G}\right)$. In addition, $\lambda_{g}\left(b_{g}\right)$ is adjointable with $\lambda_{g}\left(b_{g}\right)^{*}=\lambda_{g^{-1}}\left(b_{g}^{*}\right)$, and $\lambda_{g h}\left(b_{g} b_{h}\right)=\lambda_{g}\left(b_{g}\right) \lambda_{h}\left(b_{h}\right)$ for all $b_{g} \in B_{g}$, $b_{h} \in B_{h}$ and for all $g, h \in G$.

Definition 2.1.10. The representation $\lambda=\left\{\lambda_{g}\right\}$ of $\left(B_{g}\right)_{g \in G}$ in $\mathbb{B}\left(\ell_{2}\left(\left(B_{g}\right)_{g \in G}\right)\right)$ constructed above is called the regular representation of $\left(B_{g}\right)_{g \in G}$. 
Corollary 2.1.11. The representation of $\left(B_{g}\right)_{g \in G}$ in its cross sectional $\mathrm{C}^{*}$-algebra $\mathrm{C}^{*}\left(\left(B_{g}\right)_{g \in G}\right)$ is injective.

Proof. It suffices to show that the regular representation of $\left(B_{g}\right)_{g \in G}$ is injective. Given $b_{g} \in B_{g}$, $b_{g} a \neq 0$ for some $a \in B_{e}$. Thus $\lambda_{g}\left(b_{g}\right)\left(j_{e}(a)\right)_{g}=b_{g} a \neq 0$, where $j_{e}(a)$ is the function in $C_{c}\left(\left(B_{g}\right)_{g \in G}\right)$ associated to $a$.

Definition 2.1.12. The reduced cross sectional $\mathrm{C}^{*}$-algebra of $\left(B_{g}\right)_{g \in G}$, denoted by $\mathrm{C}_{r}^{*}\left(\left(B_{g}\right)_{g \in G}\right)$, is the $\mathrm{C}^{*}$-subalgebra of $\mathbb{B}\left(\ell_{2}\left(\left(B_{g}\right)_{g \in G}\right)\right)$ generated by the image of the regular representation of $\left(B_{g}\right)_{g \in G}$.

By the universal property of $\mathrm{C}^{*}\left(\left(B_{g}\right)_{g \in G}\right)$, there is a unique *-homomorphism

$$
\Lambda: \mathrm{C}^{*}\left(\left(B_{g}\right)_{g \in G}\right) \rightarrow \mathrm{C}_{r}^{*}\left(\left(B_{g}\right)_{g \in G}\right)
$$

such that $\Lambda \circ j_{g}=\lambda_{g}$ for all $g \in G$. We say that $\left(B_{g}\right)_{g \in G}$ is amenable if $\Lambda$ is an isomorphism.

If $G$ is a discrete group, amenability of the trivial bundle $\left(\mathbb{C} u_{g}\right)_{g \in G}$ says that the full and reduced group $\mathrm{C}^{*}$-algebras of $G$ are canonically isomorphic. Such an isomorphism is equivalent to the standard definition of amenability for groups in terms of invariant means (see [10]). By [23, Theorem 20.7], a Fell bundle over an amenable group is always amenable. This includes, in particular, Fell bundles over discrete abelian groups. This fact will be implicitly used in the subsequent chapters.

\subsection{Topologically graded $\mathrm{C}^{*}$-algebras}

In this section, we define topologically graded $\mathrm{C}^{*}$-algebras. A topological grading arises naturally from group coactions. There is a canonical Fell bundle associated to a grading of a $\mathrm{C}^{*}$-algebra, and the full and reduced cross sectional $\mathrm{C}^{*}$-algebras associated to a Fell bundle are examples of topologically graded $\mathrm{C}^{*}$-algebras.

\subsubsection{Conditional expectation}

Definition 2.2.1. Let $B$ be a $C^{*}$-algebra and $A$ a $C^{*}$-subalgebra of $B$. A positive linear map $E: B \rightarrow A$ is a conditional expectation if $E$ is contractive and idempotent, $E(a)=a$ for all $a \in A$ and $E$ is an $A$-bimodule map, that is, $E\left(a_{1} b a_{2}\right)=a_{1} E(b) a_{2}$ for all $b \in B, a_{1}, a_{2} \in A$. It is called faithful if $E\left(b^{*} b\right)=0$ implies $b=0$.

Lemma 2.2.2. Let $\left(B_{g}\right)_{g \in G}$ be a Fell bundle. For each $g \in G$, there is a contractive linear map $E_{g}: \mathrm{C}_{r}^{*}\left(\left(B_{g}\right)_{g \in G}\right) \rightarrow \lambda_{g}\left(B_{g}\right)$ such that

$$
E_{g}\left(\lambda_{h}\left(b_{h}\right)\right)= \begin{cases}b_{h} & \text { if } h=g, \\ 0 & \text { otherwise }\end{cases}
$$

Moreover, $E_{e}$ is a faithful conditional expectation onto $\lambda_{e}\left(B_{e}\right)$.

We let $E: \mathrm{C}^{*}\left(\left(B_{g}\right)_{g \in G}\right) \rightarrow B_{e}$ be the conditional expectation given by the composition

$$
\mathrm{C}^{*}\left(\left(B_{g}\right)_{g \in G}\right) \stackrel{\Lambda}{\rightarrow} \mathrm{C}_{r}^{*}\left(\left(B_{g}\right)_{g \in G}\right) \stackrel{E_{e}}{\longrightarrow} \lambda_{e}\left(B_{e}\right),
$$

followed by the identification $\lambda_{e}: B_{e} \cong \lambda_{e}\left(B_{e}\right)$. Notice that we have also identified $B_{e}$ with its image in $\mathrm{C}^{*}\left(\left(B_{g}\right)_{g \in G}\right)$ under $j_{e}$.

Corollary 2.2.3. A Fell bundle is amenable if and only if the conditional expectation

$$
E: \mathrm{C}^{*}\left(\left(B_{g}\right)_{g \in G}\right) \rightarrow B_{e}
$$

is faithful.

Definition 2.2.4. Let $B$ be a $C^{*}$-algebra and $G$ a discrete group. Let $\left\{B_{g}\right\}_{g \in G}$ be a collection of closed subspaces of $B$. We say that $\left\{B_{g}\right\}_{g \in G}$ is a grading for $B$ if, for all $g, h \in G$, one has

(i) $B_{g}^{*}=B_{g^{-1}}$, 
(ii) $B_{g} B_{h} \subseteq B_{g h}$,

(iii) $\left\{B_{g}\right\}_{g \in G}$ is linearly independent and $\bigoplus_{g \in G} B_{g}$ is a dense subspace of $B$.

A graded $\mathrm{C}^{*}$-algebra is a $\mathrm{C}^{*}$-algebra with a fixed grading.

Remark 2.2.5. Given a $G$-graded $C^{*}$-algebra $B$, then $\left(B_{g}\right)_{g \in G}$ is a Fell bundle with the operations inherited from $B$.

Definition 2.2.6. A grading $\left\{B_{g}\right\}_{g \in G}$ for a $\mathrm{C}^{*}$-algebra $B$ is a topological grading if there exists a conditional expectation $E: B \rightarrow B_{e}$ vanishing on $B_{g}$ for all $g \neq e$.

Example 2.2.7. Let $\left(B_{g}\right)_{g \in G}$ be a Fell bundle. By Lemma 2.2.2 its full and reduced cross sectional $\mathrm{C}^{*}$-algebras are topologically graded by the images of $\left(B_{g}\right)_{g \in G}$ under $j=\left\{j_{g}\right\}_{g \in G}$ and $\lambda=\left\{\lambda_{g}\right\}_{g \in G}$, respectively.

Example 2.2.8. Let $B$ be a $\mathrm{C}^{*}$-algebra equipped with a continuous action $\alpha: \mathbb{T} \rightarrow \operatorname{Aut}(B)$, where continuity here means that the map $z \mapsto \alpha_{z}(b)$ is continuous for all $b \in B$. For each $n \in \mathbb{Z}$, set

$$
B_{n}=\left\{b \in B \mid \alpha_{z}(b)=z^{n} b, \forall z \in \mathbb{T}\right\} .
$$

Then $\left\{B_{n}\right\}_{n \in \mathbb{Z}}$ is a topological grading for $B$. More generally, if $\Gamma$ is a compact abelian group and $\alpha: \Gamma \rightarrow \operatorname{Aut}(B)$ is a continuous action, then $B$ is a topologically $\widehat{\Gamma}$-graded $\mathrm{C}^{*}$-algebra, where $\widehat{\Gamma}$ is the dual group of $\Gamma]^{1}$ The spectral subspace at $g \in \widehat{\Gamma}$ is

$$
B_{g}=\left\{b \in B \mid \alpha_{\gamma}(b)=g(\gamma) b, \forall \gamma \in \Gamma\right\}
$$

and the continuous projection onto $B_{g}$ is given by

$$
E_{g}(b)=\int_{\Gamma} \overline{g(\eta)} \alpha_{\eta}(b) \mathrm{d} \mu
$$

for $b \in B$ and $g \in \widehat{\Gamma}$. See 20 for further details.

Theorem 2.2.9 (23, Theorem 19.1]). Let $\left\{B_{g}\right\}_{g \in G}$ be a topological grading for a $\mathrm{C}^{*}$-algebra B. Let $\left(B_{g}\right)_{g \in G}$ be the associated Fell bundle. There is a unique surjective ${ }^{*}$-homomorphism $\psi: B \rightarrow$ $\mathrm{C}_{r}^{*}\left(\left(B_{g}\right)_{g \in G}\right)$ such that $\psi\left(b_{g}\right)=\lambda_{g}\left(b_{g}\right)$ for all $g \in G$.

The previous theorem tells us that the reduced cross sectional $\mathrm{C}^{*}$-algebra $\mathrm{C}_{r}^{*}\left(\left(B_{g}\right)_{g \in G}\right)$ has a co-universal property: it is the smallest topologically graded $\mathrm{C}^{*}$-algebra whose associated Fell bundle coincides with $\left(B_{g}\right)_{g \in G}$.

\subsubsection{Discrete coactions}

Let $G$ be a discrete group. Let $\delta_{G}$ be the *-homomorphism $\mathrm{C}^{*}(G) \rightarrow \mathrm{C}^{*}(G) \otimes \mathrm{C}^{*}(G)$ defined by $\delta_{G}\left(u_{g}\right)=u_{g} \otimes u_{g}$, where $u_{g}$ denotes the image of $g \in G$ under the canonical group homomorphism $G \rightarrow$ $U\left(\mathrm{C}^{*}(G)\right)$. A (full) coaction of $G$ on a $\mathrm{C}^{*}$-algebra $A$ is a nondegenerate and injective *-homomorphism $\delta: A \rightarrow A \otimes \mathrm{C}^{*}(G)$ such that

$$
\left(\delta \otimes \operatorname{id}_{\mathrm{C}^{*}(G)}\right) \delta=\left(\operatorname{id}_{A} \otimes \delta_{G}\right) \delta .
$$

The triple $(A, G, \delta)$ is referred to as a coaction. See, for instance, [18, Definition A.21] and also [50]. Replacing $\mathrm{C}^{*}(G)$ by $\mathrm{C}_{r}^{*}(G)$ and adapting the coaction identity accordingly, we obtain what is called a reduced coaction [49. Here we will only use full coactions. So we will omit the term "full". The following is a key tool for the development of our main results.

Proposition 2.2.10. Let $(A, G, \delta)$ be a coaction. Then A carries a topological G-grading. The corresponding spectral subspace at $g \in G$ is

$$
A_{g}=\left\{a \in A \mid \delta(a)=a \otimes u_{g}\right\}
$$

\footnotetext{
${ }^{1}$ Recall that the dual group of a locally compact abelian group $\Gamma$, denoted by $\widehat{\Gamma}$, is the group of continuous homomorphisms from $\Gamma$ into the unit circle $\mathbb{T}$. The dual group of $\Gamma$ is discrete if and only if $\Gamma$ is compact (see 25 ).
} 
Proof. Clearly $A_{g} A_{h} \subseteq A_{g h}$ and $A_{g}^{*}=A_{g^{-1}}$ because $\delta$ is a ${ }^{*}$-homomorphism. If $a=\sum_{i=1}^{n} a_{g_{i}}=0$, then

$$
\delta(a)=\sum_{i=1}^{n} a_{g_{i}} \otimes u_{g_{i}}=0
$$

implies $E_{g_{i}}(\delta(a))=a_{g_{i}} \otimes u_{g_{i}}=0$, where $E_{g_{i}}=\mathrm{id}_{A} \otimes \chi_{g_{i}}$ and $\chi_{g_{i}}$ denotes the contractive projection of $\mathrm{C}^{*}(G)$ onto $\mathbb{C} u_{g_{i}}$. So $a_{g_{i}}=0$ for all $i \in\{1, \ldots, n\}$.

Given $a \in A$, it follows from the coaction identity that

$$
\left(\delta \otimes \operatorname{id}_{G}\right) E_{g}(\delta(a))=E_{g}(\delta(a)) \otimes u_{g}
$$

This shows that $E_{g}(\delta(a))=a_{g} \otimes u_{g}$ for some $a_{g}$ in $A_{g}$. We claim that $\bigoplus_{g \in G} A_{g}$ is dense in $A$. Since $G$ is discrete, $\delta$ automatically satisfies $\delta(A)\left(1 \otimes \mathrm{C}^{*}(G)\right)=A \otimes \mathrm{C}^{*}(G)$ (see [8]). So we may approximate $a \otimes 1 \approx \sum_{i=1}^{n} \delta\left(a_{i}\right)\left(1 \otimes u_{g_{i}}\right)$. In addition, $\operatorname{id}_{A}=\left(\operatorname{id}_{A} \otimes 1_{G}\right) \circ \delta$ by 18, Lemma A.24], where $1_{G}: G \rightarrow \mathbb{C}$ is the homomorphism $g \mapsto 1$. Then

$$
\begin{aligned}
a=\left(\operatorname{id}_{A} \otimes 1_{G}\right)(a \otimes 1) & =\left(\operatorname{id}_{A} \otimes 1_{G}\right) E_{e}(a \otimes 1) \\
& \approx\left(\operatorname{id}_{A} \otimes 1_{G}\right)\left(\sum_{i=1}^{n} E_{e}\left(\delta\left(a_{i}\right)\left(1 \otimes u_{g_{i}}\right)\right)\right) \\
& =\left(\operatorname{id}_{A} \otimes 1_{G}\right)\left(\sum_{i=1}^{n} E_{g_{i}^{-1}}\left(\delta\left(a_{i}\right)\right)\right) \in \bigoplus_{g \in G} A_{g} .
\end{aligned}
$$

Now we see that $\left(\operatorname{id}_{A} \otimes 1_{G}\right) \circ E_{g} \circ \delta$ gives a continuous projection onto $A_{g}$ that vanishes on $A_{h}$ for $h \neq g$. Hence $\left\{A_{g}\right\}_{g \in G}$ is a topological grading for $A$.

If $(A, G, \delta)$ is a coaction, we refer to the corresponding spectral subspace at $e$ as the fixed-point algebra for $\delta$.

Definition 2.2.11 ([18, Definition A.45]). Let $(A, G, \delta)$ and $(B, G, \gamma)$ be coactions. We say that a *-homomorphism $\psi: A \rightarrow B$ is $\delta$ - $\gamma$ equivariant if $\left(\psi \otimes \operatorname{id}_{G}\right) \circ \delta=\gamma \circ \psi$.

Proposition 2.2.12. Let $(A, G, \delta)$ be a coaction. Let $I \triangleleft A$ be an ideal satisfying $I=\bar{\bigoplus}_{g \in G} I \cap A_{g}$. Then there is a coaction $\delta_{A / I}: A / I \rightarrow A / I \otimes C^{*}(G)$ such that

$$
\left(q \otimes \operatorname{id}_{G}\right) \circ \delta=\delta_{A / I} \circ q .
$$

In particular, $q$ is a $\delta$ - $\delta_{A / I}$ equivariant ${ }^{*}$-homomorphism.

Proof. Given $q(a) \in A / I$, set $\delta_{A / I}(q(a)):=\left(q \otimes \mathrm{id}_{G}\right)(\delta(a))$. This vanishes on $I$ because it vanishes on $I \cap A_{g}$ for all $g \in G$ and $I$ is generated by its intersection with the spectral subspaces. It is also injective because $\left(\operatorname{id}_{A} \otimes 1_{G}\right) \circ \delta=\operatorname{id}_{A}$ gives $q(a) \in \operatorname{ker} \delta_{A / I}$ if and only if $a$ belongs to $I$. This satisfies the coaction identity because $\delta$ does so. That $\delta_{A / I}$ is a nondegenerate *homomorphism is then clear. 


\section{Chapter 3}

\section{A bicategorical interpretation for relative Cuntz-Pimsner algebras}

In this chapter, we generalise a bicategorical interpretation for Cuntz-Pimsner algebras associated to proper correspondences provided by Albandik and Meyer in [6]. Our approach does not require properness and, in addition, applies to all relative Cuntz-Pimsner algebras of single correspondences. This also extends ideas of Schweizer in [54]. We prove that the construction of relative Cuntz-Pimsner algebras yields a reflector from a certain bicategory of correspondences into a sub-bicategory of Hilbert bimodules. Roughly speaking, this shows that the passage from a correspondence $\mathcal{E}: A \leadsto A$ to the Hilbert bimodule associated to a relative Cuntz-Pimsner algebra of $\mathcal{E}$ gives the best approximation of $\mathcal{E}$ by a Hilbert bimodule, satisfying a certain property concerning the underlying ideal.

This chapter is essentially [40]. Here we have included Examples 3.1.20, 3.1.21 and 3.3.9 and also a few basic results on correspondences in Subsection 3.1.1. We will often invoke results from this chapter concerning correspondences and relative Cuntz-Pimsner algebras.

\subsection{Preliminaries}

In this section, we recall basic results on correspondences, Cuntz-Pimsner algebras, and their gauge action and Fell bundle structure. We correct and generalise an idea by Schweizer on the functoriality of Cuntz-Pimsner algebras for covariant correspondences.

\subsubsection{Correspondences}

Let $B$ be a $\mathrm{C}^{*}$-algebra and let $\mathcal{F}_{1}, \mathcal{F}_{2}$ be Hilbert $B$-modules. Let $\mathbb{B}\left(\mathcal{F}_{1}, \mathcal{F}_{2}\right)$ be the space of adjointable operators from $\mathcal{F}_{1}$ to $\mathcal{F}_{2}$. Let $|\xi\rangle\langle\eta| \in \mathbb{B}\left(\mathcal{F}_{1}, \mathcal{F}_{2}\right)$ for $\xi \in \mathcal{F}_{2}$ and $\eta \in \mathcal{F}_{1}$ be the generalised rank-1 operator defined by $|\xi\rangle\langle\eta|(\zeta):=\xi\langle\eta \mid \zeta\rangle_{B}$. Let $\mathbb{K}\left(\mathcal{F}_{1}, \mathcal{F}_{2}\right)$ be the closed linear span of $|\xi\rangle\langle\eta|$ for $\xi \in \mathcal{F}_{1}$ and $\eta \in \mathcal{F}_{2}$. Elements of $\mathbb{K}\left(\mathcal{F}_{1}, \mathcal{F}_{2}\right)$ are called compact operators. We abbreviate $\mathbb{B}(\mathcal{F}):=\mathbb{B}(\mathcal{F}, \mathcal{F})$ and $\mathbb{K}(\mathcal{F}):=\mathbb{K}(\mathcal{F}, \mathcal{F})$ if $\mathcal{F}=\mathcal{F}_{1}=\mathcal{F}_{2}$. In this case, $\mathbb{K}(\mathcal{F})$ is a closed two-sided ideal of $\mathbb{B}(\mathcal{F})$.

Lemma 3.1.1. Let $\mathcal{E}_{1} \subseteq \mathcal{F}_{1}$ and $\mathcal{E}_{2} \subseteq \mathcal{F}_{2}$ be Hilbert B-submodules. There is a unique map $\mathbb{K}\left(\mathcal{E}_{1}, \mathcal{E}_{2}\right) \rightarrow$ $\mathbb{K}\left(\mathcal{F}_{1}, \mathcal{F}_{2}\right)$ that maps $|\xi\rangle\langle\eta| \in \mathbb{K}\left(\mathcal{E}_{1}, \mathcal{E}_{2}\right)$ to $|\xi\rangle\langle\eta| \in \mathbb{K}\left(\mathcal{F}_{1}, \mathcal{F}_{2}\right)$ for all $\xi \in \mathcal{E}_{2}, \eta \in \mathcal{E}_{1}$. This map is injective.

Definition 3.1.2. Let $A$ and $B$ be $\mathrm{C}^{*}$-algebras. A correspondence from $A$ to $B$ is a Hilbert $B$-module $\mathcal{F}$ with a nondegenerate left action of $A$ through a ${ }^{*}$-homomorphism $\varphi: A \rightarrow \mathbb{B}(\mathcal{F})$. A correspondence is proper if $\varphi(A) \subseteq \mathbb{K}(\mathcal{F})$. It is faithful if $\varphi$ is injective. We write $\mathcal{F}: A \leadsto B$ to say that $\mathcal{F}$ is a correspondence from $A$ to $B$.

Definition 3.1.3. A Hilbert $A, B$-bimodule is a (right) Hilbert $B$-module $\mathcal{F}$ with a left Hilbert $A$-module structure $\left\langle\langle\cdot \mid \cdot\rangle_{A}\right.$ such that $\langle\langle\xi \mid \eta\rangle\rangle_{A} \zeta=\xi\langle\eta \mid \zeta\rangle_{B}$ for all $\xi, \eta, \zeta \in \mathcal{F}$.

If $\mathcal{F}$ is a Hilbert $A, B$-bimodule, then $A$ acts by adjointable operators on $\mathcal{F}$ and $B$ acts by adjointable operators for the left Hilbert $A$-module structure, that is, $\langle\langle\xi b \mid \eta\rangle\rangle_{A}=\left\langle\left\langle\xi \mid \eta b^{*}\right\rangle\right\rangle_{A}$ for all $\xi, \eta \in \mathcal{F}$ and all $b \in B$. In particular, $\mathcal{E}$ is an $A, B$-bimodule. The next lemma characterises which correspondences may be enriched to Hilbert bimodules: 


\section{A BICATEGORICAL INTERPRETATION FOR RELATIVE CUNTZ-PIMSNER ALGEBRAS}

Lemma 3.1.4 (see [18, Example 1.6]). A correspondence $\mathcal{F}: A \leadsto B$ carries a Hilbert $A, B$-bimodule structure if and only if there is an ideal $I \triangleleft A$ such that the left action on $\mathcal{F}$ restricts to $a^{*}$-isomorphism $I \cong \mathbb{K}(\mathcal{F})$. In this case, the ideal $I$ and the left inner product are unique, and $I=\langle\langle\mathcal{F} \mid \mathcal{F}\rangle\rangle_{A}$.

Definition 3.1.5. Let $\mathcal{F}_{1}, \mathcal{F}_{2}: A \leadsto B$ be $\mathrm{C}^{*}$-correspondences. We say that an $A, B$-bimodule map $w: \mathcal{F}_{1} \Rightarrow \mathcal{F}_{2}$ is an isometry if $\langle w(\xi) \mid w(\eta)\rangle=\langle\xi \mid \eta\rangle$ for all $\xi, \eta \in \mathcal{F}_{1}$. We say that $w$ is a correspondence isomorphism if it is unitary. We write " $\Rightarrow$ " because these isomorphisms are the 2-arrows in bicategories that we are going to construct.

Proposition 3.1.6. Let $\mathcal{F}$ be a Hilbert $A, B$-bimodule. There are canonical correspondence isomorphisms $\mathcal{F} \otimes_{B} \mathcal{F}^{*} \cong\langle\langle\mathcal{F} \mid \mathcal{F}\rangle\rangle$ and $\mathcal{F}^{*} \otimes_{A} \mathcal{F} \cong\langle\mathcal{F} \mid \mathcal{F}\rangle$. These are defined on elementary tensors by

$$
\xi \otimes \eta^{*} \mapsto\langle\langle\xi \mid \eta\rangle\rangle, \quad \xi^{*} \otimes \eta \mapsto\langle\xi \mid \eta\rangle .
$$

Let $\mathcal{F}$ be a Hilbert $B$-module and let $\varphi: A \rightarrow \mathbb{B}(\mathcal{F})$ be a ${ }^{*}$-homomorphism. For $\xi \in \mathcal{E}$, we define an operator

$$
T_{\xi}: \mathcal{F} \rightarrow \mathcal{E} \otimes_{\varphi} \mathcal{F}, \quad \eta \mapsto \xi \otimes \eta .
$$

It is adjointable with $T_{\xi}^{*}(\zeta \otimes \eta)=\varphi(\langle\xi \mid \zeta\rangle) \eta$ on elementary tensors, see [48. Hence

$$
T_{\zeta}^{*} T_{\xi}=\varphi(\langle\zeta \mid \xi\rangle), \quad T_{\xi} T_{\zeta}^{*}=|\xi\rangle\langle\zeta| \otimes 1,
$$

where $|\xi\rangle\langle\zeta| \otimes 1$ is the image of $|\xi\rangle\langle\zeta|$ under the canonical map $\mathbb{B}(\mathcal{E}) \rightarrow \mathbb{B}\left(\mathcal{E} \otimes_{\varphi} \mathcal{F}\right), T \mapsto T \otimes 1$. Hence the operator $T_{\xi}$ for $\xi \in \mathcal{E}$ is compact if and only if $\varphi(\langle\xi \mid \xi\rangle)=T_{\xi}^{*} T_{\xi}$ is compact.

Lemma 3.1.7 ([4, Corollary 3.7]). Let $J:=\varphi^{-1}(\mathbb{K}(\mathcal{F})) \triangleleft A$ and let $T \in \mathbb{K}(\mathcal{E})$. The operator $T \otimes 1$ on $\mathcal{E} \otimes_{A} \mathcal{F}$ is compact if and only if $T \in \mathbb{K}(\mathcal{E} \cdot J)$ (see Lemma 3.1.1 for the inclusion $\mathbb{K}(\mathcal{E} \cdot J) \subseteq \mathbb{K}(\mathcal{E})$ ).

In particular, if $\varphi(A) \subseteq \mathbb{K}(\mathcal{F})$, then $T \otimes 1 \in \mathbb{K}\left(\mathcal{E} \otimes_{\varphi} \mathcal{F}\right)$ for all $T \in \mathbb{K}(\mathcal{E})$. So an internal tensor product of proper correspondences is again proper.

We will often use the following result.

Lemma 3.1.8 ([48, Lemma 3.5]). Let $\mathcal{E}$ be a Hilbert A-module and $I \triangleleft A$ a closed two-sided ideal. Then

(i) The set $\{\xi \in \mathcal{E} \mid\langle\xi \mid \xi\rangle \in I\}$ is precisely the set $\mathcal{E} \cdot I$ of elements of the form $\zeta \cdot c$ for some $c \in I$.

(ii) $\mathcal{E} I$ is a submodule of $\mathcal{E}$ isomorphic to $\mathcal{E} \otimes_{A} I$.

(iii) the map $\mathbb{K}(\mathcal{E}) \rightarrow M(\mathbb{K}(\mathcal{E} I)) \cong \mathbb{B}(\mathcal{E} I)$ coming from the inclusion $\mathbb{K}(\mathcal{E} I) \hookrightarrow \mathbb{K}(\mathcal{E})$ coincides with the map $T \mapsto T \otimes 1$ from $\mathbb{K}(\mathcal{E})$ to $\mathbb{K}\left(\mathcal{E} \otimes_{A} I\right)$. Here $\mathbb{K}(\mathcal{E} I)$ is viewed as the closure in $\mathbb{K}(\mathcal{E})$ of

$$
\operatorname{span}\{|\xi\rangle\langle\eta|\in \mathbb{K}(\mathcal{E})| \xi, \eta \in \mathcal{E} I\} .
$$

The next lemma states that the invertible arrows in the correspondence bicategory of Example B.1.4 are precisely the Morita equivalences.

Lemma 3.1.9 ([18, Lemma 2.4]). Let $\mathcal{E}: A \leadsto B$ be a correspondence. Suppose that there exists a correspondence $\mathcal{F}: B \leadsto A$ with correspondence isomorphisms

$$
\mathcal{E} \otimes_{B} \mathcal{F} \cong A, \quad \mathcal{F} \otimes_{A} \mathcal{E} \cong B
$$

Then $\mathcal{E}$ is an imprimitivity $A, B$-bimodule and $\mathcal{F} \cong \mathcal{E}^{*}$.

\subsection{2 $\mathrm{C}^{*}$-algebras of correspondences}

Let $\mathcal{E}: A \leadsto A$ be a correspondence over $A$. Let $\varphi: A \rightarrow \mathbb{B}(\mathcal{E})$ be the left action. Let $\mathcal{E}^{\otimes n}$ be the $n$-fold tensor product of $\mathcal{E}$ over $A$. By convention, $\mathcal{E}^{\otimes 0}:=A$. Let $\mathcal{E}^{+}:=\bigoplus_{n=0}^{\infty} \mathcal{E}^{\otimes n}$ be the Fock space of $\mathcal{E}$, see 48 . Define

$$
t_{\xi}^{n}: \mathcal{E}^{\otimes n} \rightarrow \mathcal{E}^{\otimes n+1}, \quad \eta \mapsto \xi \otimes \eta,
$$

for $n \geq 0$ and $\xi \in \mathcal{E}$; this is the operator $T_{\xi}$ above for $\mathcal{F}=\mathcal{E}^{\otimes n}$. The operators $t_{\xi}^{n}$ combine to an operator $t_{\xi} \in \mathbb{B}\left(\mathcal{E}^{+}\right)$, that is, $\left.t_{\xi}\right|_{\mathcal{E} \otimes n}=t_{\xi}^{n}$. Let $\varphi^{+}: A \rightarrow \mathbb{B}\left(\mathcal{E}^{+}\right)$be the obvious representation by block diagonal operators and let $t^{+}: \mathcal{E} \rightarrow \mathbb{B}\left(\mathcal{E}^{+}\right)$be the linear map $\xi \mapsto t_{\xi}$. 
Definition 3.1.10. The Toeplitz $\mathrm{C}^{*}$-algebra $\mathcal{T}_{\mathcal{E}}$ of $\mathcal{E}$ is the $\mathrm{C}^{*}$-subalgebra of $\mathbb{B}\left(\mathcal{E}^{+}\right)$generated by $\varphi^{+}(A)+t^{+}(\mathcal{E})$.

Let $J$ be an ideal of $A$ with $\varphi(J) \subseteq \mathbb{K}(\mathcal{E})$. Let $P_{0}$ be the projection in $\mathbb{B}\left(\mathcal{E}^{+}\right)$that is the identity on $A \subseteq \mathcal{E}^{+}$and that vanishes on $\mathcal{E}^{\otimes n}$ for $n \geq 1$. Then $J_{0}:=\varphi^{+}(J) P_{0}$ is contained in $\mathcal{T}_{\mathcal{E}}$. The ideal in $\mathcal{T}_{\mathcal{E}}$ generated by $J_{0}$ is equal to $\mathbb{K}\left(\mathcal{E}^{+} J\right) \subseteq \mathbb{K}\left(\mathcal{E}^{+}\right)$.

Definition 3.1.11 (41, Definition 2.18]). The relative Cuntz-Pimsner algebra $\mathcal{O}_{J, \mathcal{E}}$ of $\mathcal{E}$ with respect to $J$ is $\mathcal{T}_{\mathcal{E}} / \mathbb{K}\left(\mathcal{E}^{+} J\right)$.

The following three cases are particularly important. First, if $J=\{0\}$, then $\mathcal{O}_{J, \mathcal{E}}$ is the Toeplitz $\mathrm{C}^{*}$-algebra $\mathcal{T}_{\mathcal{E}}$. Secondly, if $J=\varphi^{-1}(\mathbb{K}(\mathcal{E}))$ and $\varphi$ is injective, then $\mathcal{O}_{J, \mathcal{E}}$ is the algebra $\tilde{\mathcal{O}}_{\mathcal{E}}$ defined by Pimsner [48]. Third, if $J$ is Katsura's ideal

$$
I_{\mathcal{E}}:=\varphi_{\mathcal{E}}^{-1}(\mathbb{K}(\mathcal{E})) \cap\left(\operatorname{ker} \varphi_{\mathcal{E}}\right)^{\perp}
$$

then $\mathcal{O}_{I_{\mathcal{E}}, \mathcal{E}}$ is Katsura's Cuntz-Pimsner algebra as defined in 29$]$.

Proposition 3.1.13. Katsura's ideal $I_{\mathcal{E}}$ in 3.1 .12 is the largest ideal $J$ in $A$ with $\varphi(J) \subseteq \mathbb{K}(\mathcal{E})$ for which the canonical map $A \rightarrow \mathcal{O}_{J, \mathcal{E}}$ is injective.

Proof. That $\pi_{I_{\mathcal{E}}}$ is injective is 29 , Proposition 4.9]. The ideal $I_{\mathcal{E}}$ is maximal with this property because any ideal $J \triangleleft A$ with $\varphi(J) \subseteq \mathbb{K}(\mathcal{E})$ and $J \nsubseteq(\operatorname{ker} \varphi)^{\perp}$ must contain $a \in J$ with $\varphi(a)=0$. Then $\varphi^{+}(a) \in \varphi^{+}(J) \cdot P_{0}$ becomes 0 in $\mathcal{O}_{J, \mathcal{E}}$.

Definition 3.1.14. Let $\mathcal{E}: A \leadsto A$ be a correspondence and $B$ a $\mathrm{C}^{*}$-algebra. A representation of $\mathcal{E}$ in $B$ is a pair $(\pi, t)$, where $\pi: A \rightarrow B$ is a ${ }^{*}$-homomorphism, $t: \mathcal{E} \rightarrow B$ is a linear map, and

(1) $\pi(a) t(\xi)=t(\varphi(a) \xi)$ for all $a \in A$ and $\xi \in \mathcal{E}$;

(2) $t(\xi)^{*} t(\eta)=\varphi\left(\langle\xi \mid \eta\rangle_{A}\right)$ for all $\xi, \eta \in \mathcal{E}$.

These conditions imply $t(\xi) \pi(a)=t(\xi a)$ for all $\xi \in \mathcal{E}$ and $a \in A$.

In particular, $\left(\varphi^{+}, t^{+}\right)$is a representation of $\mathcal{E}$ in the Toeplitz $\mathrm{C}^{*}$-algebra $\mathcal{T}_{\mathcal{E}}$. This representation is universal in the following sense:

Proposition 3.1.15. Any representation $(\pi, t)$ of $\mathcal{E}$ in a $\mathrm{C}^{*}$-algebra $B$ is of the form $\left(\tilde{\pi} \circ \varphi^{+}, \tilde{\pi} \circ t^{+}\right)$ for a unique ${ }^{*}$-homomorphism $\tilde{\pi}: \mathcal{T}_{\mathcal{E}} \rightarrow B$. Conversely, $\left(\tilde{\pi} \circ \varphi^{+}, \tilde{\pi} \circ t^{+}\right)$is a representation of $\mathcal{E}$ for any ${ }^{*}$-homomorphism $\tilde{\pi}: \mathcal{T}_{\mathcal{E}} \rightarrow B$

Lemma 3.1.16. For any representation $(\pi, t)$ of $\mathcal{E}$, there is a unique ${ }^{*}$-homomorphism $\pi^{1}: \mathbb{K}(\mathcal{E}) \rightarrow B$ with $\pi^{1}(|\xi\rangle\langle\eta|)=t_{\xi} t_{\eta}^{*}$ for all $\xi, \eta \in \mathcal{E}$.

Proposition 3.1.17 ([41, Theorem 2.19]). The representation $\tilde{\pi}$ of $\mathcal{T}_{\mathcal{E}}$ associated to a representation $(\pi, t)$ of $\mathcal{E}$ factors through the quotient $\mathcal{O}_{J, \mathcal{E}}$ of $\mathcal{T}_{\mathcal{E}}$ if and only if

$$
\pi(a)=\pi^{1}(\varphi(a)) \quad \text { for all } a \in J
$$

In this case, we call the representation covariant on $J$.

Let $\left(\pi_{J}, t_{J}\right)$ be the canonical representation of $\mathcal{E}$ in $\mathcal{O}_{J, \mathcal{E}}$. Proposition 3.1 .17 says that $\left(\pi_{J}, t_{J}\right)$ is the universal representation of $\mathcal{E}$ that is covariant on $J$.

Proposition 3.1.19. A representation $(\pi, t)$ in $B$ is covariant on $J$ if and only if $\pi(J) \subseteq t(\mathcal{E}) \cdot B$.

Proof. Let $a \in J$. Then $\pi^{1}(\varphi(a))$ is contained in the closed linear span of $t(\mathcal{E}) t(\mathcal{E})^{*}$ and hence in $t(\mathcal{E}) \cdot B$. So $\pi(a) \in t(\mathcal{E}) \cdot B$ is necessary for $\pi(a)=\pi^{1}(\varphi(a))$. Conversely, assume $\pi(a) \in t(\mathcal{E}) \cdot B$ for all $a \in J$. We have $\pi(a) \cdot t(\xi)=t(\varphi(a) \xi)=\pi^{1}(\varphi(a)) t(\xi)$ for all $\xi \in \mathcal{E}$ (see [29, Lemma 2.4]). Hence $\left(\pi(a)-\pi^{1}(\varphi(a))\right) \cdot t(\mathcal{E}) \cdot B=0$. Since $\pi\left(a^{*}\right), \pi^{1}\left(\varphi\left(a^{*}\right)\right) \in t(\mathcal{E}) \cdot B$, we get $\left(\pi(a)-\pi^{1}(\varphi(a))\right) \cdot(\pi(a)-$ $\left.\pi^{1}(\varphi(a))\right)^{*}=0$. This is equivalent to $\pi(a)=\pi^{1}(\varphi(a))$. 


\section{A BICATEGORICAL INTERPRETATION FOR RELATIVE CUNTZ-PIMSNER ALGEBRAS}

Example 3.1.20. View $\mathbb{C}^{n}$ as a correspondence over $\mathbb{C}$ in the obvious way. This is a faithful and proper correspondence. So let $J=\mathbb{C}$ and for each $i \in\{1, \ldots, n\}$, let $s_{i}$ be the image in $\mathcal{O}_{\mathbb{C}, \mathbb{C}^{n}}$ of the canonical unit vector $e_{i}$ of $\mathbb{C}^{n}$ under the universal representation. Then $s_{i}$ is an isometry. In addition, the covariance condition is equivalent to

$$
\sum_{i=1}^{n} s_{i} s_{i}^{*}=1 .
$$

Thus $\mathcal{O}_{\mathbb{C}, \mathbb{C}^{n}}$ is isomorphic to the Cuntz algebra $\mathcal{O}_{n}$.

Example 3.1.21 (Crossed products by transfer operators). Let $A$ be a unital $\mathrm{C}^{*}$-algebra and let $\alpha$ be an endomorphism of $A$. A continuous linear map $L: A \rightarrow A$ is a transfer operator for $(A, \alpha)$ if it is positive and $L(\alpha(a) b)=a L(b)$ for all $a, b \in A$. Since $A$ is spanned by its positive elements and $L$ is positive, it follows that $L(a)^{*}=L\left(a^{*}\right)$ for all $a \in A$. In particular, the identity $L(a \alpha(b))=L(a) b$ also holds.

We may associate a correspondence $A_{\alpha, L}: A \leadsto A$ to $(A, \alpha, L)$ as follows. We set $A_{\alpha, L}^{0}=A \alpha(1)$ as a complex vector space. We define a structure of $A$-module on $A_{\alpha, L}$ by $a \alpha(1) \cdot b:=a \alpha(b)$ and an $A$-valued inner product by $\langle a \alpha(1) \mid b \alpha(1)\rangle:=L\left(a^{*} b\right)$, for $a, b \in A$. This satisfies $\langle a \alpha(1) \mid b \alpha(1) \cdot c\rangle=$ $\langle a \alpha(1) \mid b \alpha(1)\rangle c$ because $L$ is a transfer operator for $(A, \alpha)$. Since $L$ is positive, the Cauchy-Schwarz inequality also holds in this context, and it follows that

$$
N:=\left\{a \alpha(1) \in A \mid L\left(a^{*} a\right)=0\right\}
$$

is a subspace of $A_{\alpha, L}^{0}$ (see Lemma A.1.3. Hence the quotient space $A_{\alpha, L}^{0} / N$ is a pre-Hilbert $A$-module. We denote by $A_{\alpha, L}$ its completion with respect to the norm coming from the $A$-valued inner product. Thus $A_{\alpha, L}$ is a Hilbert $A$-module.

Now using again that $L$ is positive, we deduce that

$$
L\left(b^{*} a^{*} a b\right) \leq\|a\|^{2} L\left(b^{*} b\right)
$$

for all $a, b \in A$. Hence left multiplication by elements of $A$ produces a nondegenerate *-homomorphism $\varphi: A \rightarrow \mathbb{B}\left(A_{\alpha, L}\right)$. This turns $A_{\alpha, L}$ into a correspondence over $A$.

Let $(\pi, t)$ be a nondegenerate representation of $A_{\alpha, L}$ in a unital $\mathrm{C}^{*}$-algebra $B$. Set $V:=t(\alpha(1))$. Then, for all $a \in A, V \pi(a)=\pi(\alpha(a)) V$. In addition,

$$
V^{*} \pi(a) V=t(\alpha(1))^{*} \pi(a) t(\alpha(1))=t(\alpha(1))^{*} t(a \alpha(1))=L(a) .
$$

Conversely, suppose that $\pi: A \rightarrow B$ is a unital ${ }^{*}$-homomorphism and $V$ is an element of $B$, so that the relations

$$
V \pi(a)=\pi(\alpha(a)) V, \quad V^{*} \pi(a) V=L(a)
$$

hold for all $a \in A$. Set $t^{\prime}(b \alpha(1)):=\pi(b) V$ for all $b \in A$. It follows that $t^{\prime}$ vanishes on $N$ and is continuous with respect to the norm on $A_{\alpha, L}^{0} / N$ because, for all $b \in B$,

$$
\|b V\|^{2}=\left\|V^{*} b^{*} b V\right\|=\left\|L\left(b^{*} b\right)\right\| .
$$

Hence it descends to a linear map $t: A_{\alpha, L} \rightarrow B$. The relations in 3.1 .22 then say that $(\pi, t)$ is a representation of $A_{\alpha, L}$. The $\mathrm{C}^{*}$-algebra $\mathcal{T}(A, \alpha, L)$ introduced in 21 is the universal unital $\mathrm{C}^{*}$-algebra generated by a copy of $A$ and an element $S$ subject to the above relations. We see that $\mathcal{T}(A, \alpha, L)$ is naturally isomorphic to the Toeplitz algebra of $A_{\alpha, L}$. The crossed product $A \rtimes_{\alpha, L} \mathbb{N}$ of $A$ by the endomorphism $\alpha$ relative to the transfer operator $L$ was defined by Exel in $[21]$ to be the quotient of $\mathcal{T}(A, \alpha, L)$ by the ideal generated by

$$
\left\{a-k \mid a \in A, k \in \overline{A S S^{*} A} \text { and } a b S=k b S, \forall b \in A\right\} .
$$

Observe that $A S S^{*} A$ corresponds to $t^{+}\left(A_{\alpha, L}\right) t^{+}\left(A_{\alpha, L}\right)^{*}$ through the above isomorphism $\mathcal{T}(A, \alpha, L) \cong$ $\mathcal{T}_{A_{\alpha, L}}$. From this we deduce that $A \rtimes_{\alpha, L} \mathbb{N}$ is isomorphic to the quotient of $\mathcal{T}_{A_{\alpha, L}}$ by the ideal generated by

$$
\left\{\varphi^{+}(a)-\left(\varphi^{+}\right)^{1}(\varphi(a)) \mid \varphi(a) \in \mathbb{K}\left(A_{\alpha, L}\right)\right\} .
$$

This is precisely the relative Cuntz-Pimsner algebra $\mathcal{O}_{J, A_{\alpha, L}}$, where $J=\varphi^{-1}\left(\mathbb{K}\left(A_{\alpha, L}\right)\right)$.

Brownlowe and Raeburn realised the crossed product $A \rtimes_{\alpha, L} \mathbb{N}$ as a relative Cuntz-Pimsner algebra 
of $A_{\alpha, L}$ in 11. With this approach, they were able to establish necessary and sufficient conditions for the universal *-homomorphism $A \rightarrow A \rtimes_{\alpha, L} \mathbb{N}$ to be an embedding. They also applied more general results concerning relative Cuntz-Pimsner algebras of correspondences to prove gauge-invariant uniqueness theorems for the crossed product $A \rtimes_{\alpha, L} \mathbb{N}[11$, Section 5].

\subsubsection{Gauge action and Fell bundle structure}

Let $\mathcal{E}: A \leadsto A$ be a correspondence and let $J \triangleleft A$ be an ideal with $\varphi(J) \subseteq \mathbb{K}(\mathcal{E})$. If $(\pi, t)$ is a representation of $\mathcal{E}$ that is covariant on $J$, then so is $(\pi, z \cdot t)$ for $z \in \mathbb{T}$. This operation on representations comes from an automorphism of the relative Cuntz-Pimsner algebra $\mathcal{O}_{J, \mathcal{E}}$ by its universal property. These automorphisms define a continuous action $\gamma$ of $\mathbb{T}$ on $\mathcal{O}_{J, \mathcal{E}}$, called the gauge action. Let

$$
\mathcal{O}_{J, \mathcal{E}}^{n}:=\left\{b \in \mathcal{O}_{J, \mathcal{E}}: \gamma_{z}(b)=z^{n} b \text { for all } z \in \mathbb{T}\right\}
$$

for $n \in \mathbb{Z}$ be the $n$th spectral subspace. These spectral subspaces form a Fell bundle over $\mathbb{Z}$, that is, $\mathcal{O}_{J, \mathcal{E}}^{n} \cdot \mathcal{O}_{J, \mathcal{E}}^{m} \subseteq \mathcal{O}_{J, \mathcal{E}}^{n+m}$ and $\left(\mathcal{O}_{J, \mathcal{E}}^{n}\right)^{*}=\mathcal{O}_{J, \mathcal{E}}^{-n}$ for all $n, m \in \mathbb{Z}$. In particular, for $J=\{0\}$ we get a gauge action on $\mathcal{T}_{\mathcal{E}}$ and corresponding spectral subspaces $\mathcal{T}_{\mathcal{E}}^{n} \subseteq \mathcal{T}_{\mathcal{E}}$. Explicitly, the gauge action on $\mathcal{T}_{\mathcal{E}}$ comes from the obvious $\mathbb{N}$-grading on $\mathcal{E}^{+}$: if $x \in \mathcal{T}_{\mathcal{E}}$, then $x \in \mathcal{T}_{\mathcal{E}}^{n}$ if and only if $x\left(\mathcal{E}^{\otimes k}\right) \subseteq \mathcal{E}^{\otimes n+k}$ for all $k \in \mathbb{N}$; this means $\left.x\right|_{\mathcal{E} \otimes k}=0$ if $k+n<0$. And $\mathcal{O}_{J, \mathcal{E}}^{n}$ is the image of $\mathcal{T}_{\mathcal{E}}^{n}$ in $\mathcal{O}_{J, \mathcal{E}}$.

Lemma 3.1.23. Let $n \in \mathbb{Z}$. The subspace $\mathcal{O}_{J, \mathcal{E}}^{n}$ in $\mathcal{O}_{J, \mathcal{E}}$ is the closed linear span of $t_{J}\left(\xi_{1}\right) t_{J}\left(\xi_{2}\right) \cdots t_{J}\left(\xi_{k}\right)$. $t_{J}^{*}\left(\eta_{l}\right) \cdots t_{J}^{*}\left(\eta_{2}\right) t_{J}^{*}\left(\eta_{1}\right)$ for $\xi_{i}, \eta_{j} \in \mathcal{E}, k-l=n$. If $n \in \mathbb{N}$, then

$$
\mathcal{O}_{J, \mathcal{E}}^{n} \cong \mathcal{E}^{\otimes n} \otimes_{A} \mathcal{O}_{J, \mathcal{E}}^{0}
$$

as a correspondence $A \leadsto \mathcal{O}_{J, \mathcal{E}}^{0}$. The Fell bundle $\left(\mathcal{O}_{J, \mathcal{E}}^{k}\right)_{k \in \mathbb{Z}}$ is semi-saturated, that is, $\mathcal{O}_{J, \mathcal{E}}^{k} \cdot \mathcal{O}_{J, \mathcal{E}}^{l}=\mathcal{O}_{J, \mathcal{E}}^{k+l}$ if $k, l \geq 0$.

Proof. Let $b \in \mathcal{O}_{J, \mathcal{E}}^{n}$ and let $\epsilon>0$. Then $b$ is $\epsilon$-close to a finite linear combination $b_{\epsilon}$ of monomials $t_{J}\left(\xi_{1}\right) t_{J}\left(\xi_{2}\right) \cdots t_{J}\left(\xi_{k}\right) \cdot t_{J}^{*}\left(\eta_{l}\right) \cdots t_{J}^{*}\left(\eta_{2}\right) t_{J}^{*}\left(\eta_{1}\right)$ with $k, l \in \mathbb{N}$. Define

$$
p_{n}(x):=\int_{\mathbb{T}} z^{-n} \gamma_{z}(x) \mathrm{d} z, \quad x \in \mathcal{O}_{J, \mathcal{E}} .
$$

This is a contractive projection from $\mathcal{O}_{J, \mathcal{E}}$ onto $\mathcal{O}_{J, \mathcal{E}}^{n}$. Since $p_{n}(b)=b$ and $\left\|p_{n}\right\| \leq 1$, we have $\left\|b-p_{n}\left(b_{\epsilon}\right)\right\| \leq \epsilon$ as well. Inspection shows that $p_{n}$ maps a monomial $t_{J}\left(\xi_{1}\right) t_{J}\left(\xi_{2}\right) \cdots t_{J}\left(\xi_{k}\right)$. $t_{J}^{*}\left(\eta_{l}\right) \cdots t_{J}^{*}\left(\eta_{2}\right) t_{J}^{*}\left(\eta_{1}\right)$ to itself if $k-l=n$ and kills it otherwise. Hence $\mathcal{O}_{J, \mathcal{E}}^{n}$ is the closed linear span of such monomials with $k-l=n$.

The monomials generating $\mathcal{O}_{J, \mathcal{E}}^{k+l}$ for $k, l \geq 0$ are obviously in $\mathcal{O}_{J, \mathcal{E}}^{k} \cdot \mathcal{O}_{J, \mathcal{E}}^{l}$. Hence the first statement immediately implies the last one. There is an isometric $A, \mathcal{O}_{J, \mathcal{E}^{-b i m o d u l e ~ m a p ~}}^{0}$

$$
\mathcal{E}^{\otimes n} \otimes_{A} \mathcal{O}_{J, \mathcal{E}}^{0} \rightarrow \mathcal{O}_{J, \mathcal{E}}^{n}, \quad \xi_{1} \otimes \cdots \otimes \xi_{n} \otimes y \mapsto t_{J}\left(\xi_{1}\right) \cdots t_{J}\left(\xi_{n}\right) \cdot y .
$$

The first statement implies that its image is dense, so it is unitary.

The Fell bundle $\left(\mathcal{O}_{J, \mathcal{E}}^{n}\right)_{n \in \mathbb{Z}}$ need not be saturated, that is, $\mathcal{O}_{J, \mathcal{E}}^{n} \cdot \mathcal{O}_{J, \mathcal{E}}^{-n}$ may differ from $\mathcal{O}_{J, \mathcal{E}}^{0}$.

Theorem 3.1.24. The relative Cuntz-Pimsner algebra is $\mathbb{T}$-equivariantly isomorphic to the crossed product of $\mathcal{O}_{J, \mathcal{E}}^{0}$ by the Hilbert $\mathcal{O}_{J, \mathcal{E}}^{0}$-bimodule $\mathcal{O}_{J, \mathcal{E}}^{1}$ and to the full or reduced section $\mathrm{C}^{*}$-algebra of the Fell bundle $\left(\mathcal{O}_{J, \mathcal{E}}^{n}\right)_{n \in \mathbb{Z}}$.

Proof. The Fell bundle $\left(\mathcal{O}_{J, \mathcal{E}}^{n}\right)_{n \in \mathbb{Z}}$ is semi-saturated by Lemma 3.1.23. Now the results of AbadieEilers-Exel [1] imply our claims.

Theorem 3.1.24 splits the construction of relative Cuntz-Pimsner algebras into two steps. The first builds the Hilbert $\mathcal{O}_{J, \mathcal{E}^{-}}^{0}$ bimodule $\mathcal{O}_{J, \mathcal{E}}^{1}$, the second takes the crossed product for this Hilbert bimodule. A Hilbert bimodule $\mathcal{G}$ on a $\mathrm{C}^{*}$-algebra $B$ is the same as a Morita-Rieffel equivalence between two ideals in $B$ or, briefly, a partial Morita-Rieffel equivalence on $B$ (this point of view is explained in [12]). The crossed product $\mathcal{O}_{J, \mathcal{E}}^{0} \rtimes \mathcal{O}_{J, \mathcal{E}}^{1}$ generalises the partial crossed product for a partial automorphism. Many results about crossed products for automorphisms extend to Hilbert bimodule crossed products. In particular, the standard criteria for simplicity and detection and separation of ideals are extended in 33 . 
Proposition 3.1.25. The following conditions are equivalent:

(1) the $\operatorname{map} \pi_{J}: A \rightarrow \mathcal{O}_{J, \mathcal{E}}^{0}$ is an isomorphism;

(2) the $\operatorname{map} \varphi: J \rightarrow \mathbb{K}(\mathcal{E})$ is an isomorphism;

(3) the correspondence $\mathcal{E}$ comes from a Hilbert bimodule and $J=I_{\mathcal{E}}$.

Proof. If $J=I_{\mathcal{E}}$ is Katsura's ideal, then everything follows from [29, Proposition 5.18]. So it remains to observe that $(1)$ and $(2)$ fail if $J \neq I_{\mathcal{E}}$. Lemma 3.1 .4 shows that $\mathcal{E}$ comes from a Hilbert bimodule if and only if there is an ideal $I$ in $A$ so that $\left.\varphi\right|_{I}: I \rightarrow \mathbb{K}(\mathcal{E})$ is an isomorphism. In this case, $I$ is the largest ideal on which $\varphi$ restricts to an injective map into $\mathbb{K}(\mathcal{E})$. So $I=I_{\mathcal{E}}$. Thus $[(2) \Longleftrightarrow(3)$

If $J \nsubseteq I_{\mathcal{E}}$, then $A \rightarrow \mathcal{O}_{J, \mathcal{E}}$ is not injective by Proposition 3.1.13. So (1) implies $J \subseteq I_{\mathcal{E}}$. If $J \subseteq I_{\mathcal{E}}$ and (1) holds, then the map $A \rightarrow \mathcal{O}_{I_{\mathcal{E}}, \mathcal{E}}^{0}$ is still surjective because $\mathcal{O}_{I_{\mathcal{E}}, \mathcal{E}}$ is a quotient of $\mathcal{O}_{J, \mathcal{E}}$, and it is also injective by Proposition 3.1.13 Hence $\mathcal{O}_{I_{\mathcal{E}}, \mathcal{E}}=\mathcal{O}_{J, \mathcal{E}}$. This implies $\mathbb{K}\left(\mathcal{E}^{+} I_{\mathcal{E}}\right)=\mathbb{K}\left(\mathcal{E}^{+} J\right)$ and hence $I_{\mathcal{E}}=J$ because of the direct summand $A$ in $\mathcal{E}^{+}$.

Proposition 3.1.26. Let $\mathcal{G}$ be a Hilbert B-bimodule and let $I_{\mathcal{G}}$ be Katsura's ideal for $\mathcal{G}$. Then $\mathcal{O}_{I_{\mathcal{G}}, \mathcal{G}} \cong B \rtimes \mathcal{G} \mathbb{T}$-equivariantly.

Proof. Theorem 3.1 .24 identifies $\mathcal{O}_{I_{\mathcal{G}}, \mathcal{G}} \cong \mathcal{O}_{I_{\mathcal{G}}, \mathcal{G}}^{0} \rtimes \mathcal{O}_{I_{\mathcal{G}} \mathcal{G}}^{1}$. Proposition 3.1 .25 gives $B \cong \mathcal{O}_{I_{\mathcal{G}}, \mathcal{G}}^{0}$, and the isomorphism $\mathcal{O}_{I_{\mathcal{G}}, \mathcal{G}}^{1} \cong \mathcal{G} \otimes_{B} \mathcal{O}_{I_{\mathcal{G}}, \mathcal{G}}^{0}$ from Lemma 3.1 .23 implies that $\mathcal{G} \cong \mathcal{O}_{I_{\mathcal{G}}, \mathcal{G}}^{1}$ as a Hilbert $B$-bimodule.

\subsubsection{Functoriality of relative Cuntz-Pimsner algebras}

Schweizer [54] has defined "covariant homomorphisms" and "covariant correspondences" between selfcorrespondences and has asserted that they induce *-homomorphisms and correspondences between the associated Toeplitz and absolute Cuntz-Pimsner algebras. For the proof of functoriality for covariant correspondences he refers to a preprint that never got published. In fact, there are some technical pitfalls. We correct his statement here, and also add a condition to treat relative Cuntz-Pimsner algebras.

Throughout this subsection, let $\mathcal{E}: A \leadsto A$ and $\mathcal{G}: B \leadsto B$ be correspondences and let $J_{A} \subseteq$ $\varphi^{-1}(\mathbb{K}(\mathcal{E}))$ and $J_{B} \subseteq \varphi^{-1}(\mathbb{K}(\mathcal{G}))$ be ideals.

Definition 3.1.27. A covariant correspondence from $\left(A, \mathcal{E}, J_{A}\right)$ to $\left(B, \mathcal{G}, J_{B}\right)$ is a pair $(\mathcal{F}, V)$, where $\mathcal{F}$ is a correspondence $A \leadsto B$ with $J_{A} \cdot \mathcal{F} \subseteq \mathcal{F} \cdot J_{B}$ and $V$ is a correspondence isomorphism $\mathcal{E} \otimes_{A} \mathcal{F} \Rightarrow$ $\mathcal{F} \otimes_{B} \mathcal{G}$. A covariant correspondence is proper if $\mathcal{F}$ is proper.

Proposition 3.1.28. A proper covariant correspondence $(\mathcal{F}, V)$ from $\left(A, \mathcal{E}, J_{A}\right)$ to $\left(B, \mathcal{G}, J_{B}\right)$ induces a proper $\mathbb{T}$-equivariant correspondence $\mathcal{O}_{\mathcal{F}, V}: \mathcal{O}_{J_{A}, \mathcal{E}} \leadsto \mathcal{O}_{J_{B}, \mathcal{G}}$

Schweizer [54 claims this also for non-proper correspondences, and he allows $V$ to be a nonadjointable isometry. In fact, a pair $(\mathcal{F}, V)$ where $V$ is only a non-adjointable isometry induces a correspondence between the Toeplitz $\mathrm{C}^{*}$-algebras. It is unclear, however, when this correspondence descends to one between the absolute or relative Cuntz-Pimsner algebras. And we need $\mathcal{F}$ to be proper. Alternatively, we may require $\mathcal{E}$ instead of $\mathcal{F}$ to be proper. This situation is treated in [6].

Proof. We use the canonical *-homomorphism $\pi_{J_{B}}: B \rightarrow \mathcal{O}_{J_{B}, \mathcal{G}}$ to view $\mathcal{O}_{J_{B}, \mathcal{G}}$ as a proper correspondence $B \leadsto \mathcal{O}_{J_{B}, \mathcal{G}}$. Thus $\mathcal{F}_{\mathcal{O}}:=\mathcal{F} \otimes_{B} \mathcal{O}_{J_{B}, \mathcal{G}}$ becomes a proper correspondence $A \sim \mathcal{O}_{J_{B}, \mathcal{G}}$, that is, a Hilbert $\mathcal{O}_{J_{B}, \mathcal{G}}$-module with a representation $\pi: A \rightarrow \mathbb{K}\left(\mathcal{F}_{\mathcal{O}}\right)$. The $\mathbb{T}$-action on $\mathcal{O}_{J_{B}, \mathcal{G}}$ induces a $\mathbb{T}$-action on $\mathcal{F}_{\mathcal{O}}$ because $\pi_{J_{B}}(B) \subseteq \mathcal{O}_{J_{B}, \mathcal{G}}^{0}$. We are going to define a map $t: \mathcal{E} \rightarrow \mathbb{K}\left(\mathcal{F}_{\mathcal{O}}\right)$ such that $(\pi, t)$ is a representation of $(A, \mathcal{E})$ on $\mathcal{F}_{\mathcal{O}}$ that is covariant on $J_{A}$. Then Proposition 3.1 .17 yields a representation $\tilde{\pi}: \mathcal{O}_{J_{A}, \mathcal{E}} \rightarrow \mathbb{K}\left(\mathcal{F}_{\mathcal{O}}\right)$. This is the desired correspondence $\mathcal{O}_{J_{A}, \mathcal{E}} \leadsto \mathcal{O}_{J_{B}, \mathcal{G}}$.

There is an isometry $\mu_{\mathcal{G}}: \mathcal{G} \otimes_{B} \mathcal{O}_{J_{B}, \mathcal{G}} \Rightarrow \mathcal{O}_{J_{B}, \mathcal{G}}, \zeta \otimes y \mapsto t^{+}(\zeta) \cdot y$, of correspondences $B \leadsto \mathcal{O}_{J_{B}, \mathcal{G}}$. Usually, it is not unitary. We define an isometry

$$
V^{!}: \mathcal{E} \otimes_{A} \mathcal{F}_{\mathcal{O}}=\mathcal{E} \otimes_{A} \mathcal{F} \otimes_{B} \mathcal{O}_{J_{B}, \mathcal{G}} \stackrel{V \otimes 1}{\Longrightarrow} \mathcal{F} \otimes_{B} \mathcal{G} \otimes_{B} \mathcal{O}_{J_{B}, \mathcal{G}} \stackrel{1 \otimes \mu_{\mathcal{G}}}{\Longrightarrow} \mathcal{F} \otimes_{B} \mathcal{O}_{J_{B}, \mathcal{G}}=\mathcal{F}_{\mathcal{O}}
$$

It yields a map $t$ from $\mathcal{E}$ to the space of bounded operators on $\mathcal{F}_{\mathcal{O}}$ by $t(\xi)(\eta):=V^{!}(\xi \otimes \eta)$. To show that $t(\xi)$ is adjointable, we need that $\mathcal{F}_{\mathcal{O}}$ is a proper correspondence $A \leadsto \mathcal{O}_{J_{B}, \mathcal{G}}$ : then $T_{\xi} \in \mathbb{K}\left(\mathcal{F}_{\mathcal{O}}, \mathcal{E} \otimes{ }_{A} \mathcal{F}_{\mathcal{O}}\right)$, and composition with $V^{!}$maps this into $\mathbb{K}\left(\mathcal{F}_{\mathcal{O}}\right)$ by Lemma 3.1.1 So even $t(\xi) \in \mathbb{K}\left(\mathcal{F}_{\mathcal{O}}\right)$ for all $\xi \in \mathcal{E}$. 
We claim that the pair $(\pi, t)$ is a representation. We have $\pi(a) t(\xi)=t(\varphi(a) \xi)$ because $V^{!}$is a left $A$-module map. And $t\left(\xi_{1}\right)^{*} t\left(\xi_{2}\right)=\pi\left(\left\langle\xi_{1} \mid \xi_{2}\right\rangle\right)$ holds because

$$
\left\langle t\left(\xi_{1}\right) \eta_{1} \mid t\left(\xi_{2}\right) \eta_{2}\right\rangle=\left\langle V^{!}\left(\xi_{1} \otimes \eta_{1}\right) \mid V^{!}\left(\xi_{2} \otimes \eta_{2}\right)\right\rangle=\left\langle\xi_{1} \otimes \eta_{1} \mid \xi_{2} \otimes \eta_{2}\right\rangle=\left\langle\eta_{1} \mid \pi\left(\left\langle\xi_{1} \mid \xi_{2}\right\rangle\right) \eta_{2}\right\rangle
$$

If $J_{A}=0$, then we are done at this point, and we have not yet used that $V$ is unitary. So the Toeplitz $\mathrm{C}^{*}$-algebra of a correspondence remains functorial for proper covariant correspondences where $V$ is not unitary.

It remains to prove that $\pi$ is covariant on $J_{A}$. By Proposition 3.1.19 this is equivalent to $\pi\left(J_{A}\right)\left(\mathcal{F}_{\mathcal{O}}\right) \subseteq t(\mathcal{E})\left(\mathcal{F}_{\mathcal{O}}\right)$. And $J_{B} \cdot \mathcal{O}_{J_{B}, \mathcal{G}} \subseteq t_{J_{B}}(\mathcal{G}) \cdot \mathcal{O}_{J_{B}, \mathcal{G}}$ holds because the canonical representation of $(B, \mathcal{G})$ on $\mathcal{O}_{J_{B}, \mathcal{G}}$ is covariant on $J_{B}$. Since $J_{A} \cdot \mathcal{F} \subseteq \mathcal{F} \cdot J_{B}$ by assumption,

$$
J_{A} \cdot \mathcal{F}_{\mathcal{O}} \subseteq \mathcal{F} \otimes J_{B} \cdot \mathcal{O}_{J_{B}, \mathcal{G}} \subseteq \mathcal{F} \otimes t_{J_{B}}(\mathcal{G}) \cdot \mathcal{O}_{J_{B}, \mathcal{G}}=\left(1 \otimes \mu_{\mathcal{G}}\right)\left(\mathcal{F} \otimes_{B} \mathcal{G} \otimes_{B} \mathcal{O}_{J_{B}, \mathcal{G}}\right)
$$

Since $V$ is unitary, we may rewrite this further as $V^{!}\left(\mathcal{E} \otimes_{A} \mathcal{F} \otimes_{B} \mathcal{O}_{J_{B}, \mathcal{G}}\right)=t(\mathcal{E}) \cdot \mathcal{F}_{\mathcal{O}}$. This finishes the proof that $(\pi, t)$ is covariant on $J_{A}$. The operators $t(\xi)$ for $\xi \in \mathcal{E}$ are homogeneous of degree 1 for the $\mathbb{T}$-action. Thus $\tilde{\pi}$ is $\mathbb{T}$-equivariant.

Example 3.1.29. Let $A=B$ and $J=J_{A}=J_{B} \neq\{0\}$ and let $\mathcal{E} \subseteq \mathcal{G}$ be an $A$-invariant Hilbert submodule. Then the identity correspondence $\mathcal{F}=A$ with the inclusion map $\mathcal{E} \otimes_{A} \mathcal{F} \cong \mathcal{E} \hookrightarrow \mathcal{G} \cong \mathcal{F} \otimes_{A} \mathcal{G}$ is a covariant correspondence in the notation of Schweizer. There is indeed a canonical *-homomorphism $\mathcal{T}_{\mathcal{E}} \rightarrow \mathcal{T}_{\mathcal{G}}$. But it need not descend to the relative Cuntz-Pimsner algebras because $\varphi_{\mathcal{G}}(a) \in \mathbb{K}(\mathcal{G})$ for $a \in J$ need not be the extension of $\varphi_{\mathcal{E}}(a) \in \mathbb{K}(\mathcal{E})$ given by Lemma 3.1.1. So the Cuntz-Pimsner covariance conditions for $\mathcal{O}_{J, \mathcal{E}}$ and $\mathcal{O}_{J, \mathcal{G}}$ may be incompatible. We ask $V$ to be unitary to avoid this problem.

Lemma 3.1.30. Turn $\mathcal{O}_{J, \mathcal{E}}^{0}$, into a proper $\mathrm{C}^{*}$-correspondence $A \leadsto \mathcal{O}_{J, \mathcal{E}}^{0}$ with the obvious left action of $A$. The proper correspondence $\mathcal{O}_{J, \mathcal{E}}^{0}: A \leadsto \mathcal{O}_{J, \mathcal{E}}^{0}$ with the isomorphism from Lemma 3.1 .23 is a proper covariant correspondence from $\mathcal{E}: A \leadsto A$ with the ideal $J$ to $\mathcal{O}_{J, \mathcal{E}}^{1}: \mathcal{O}_{J, \mathcal{E}}^{0} \leadsto \mathcal{O}_{J, \mathcal{E}}^{0}$ with Katsura's ideal $I_{\mathcal{O}_{J, \mathcal{E}}^{1}}$.

Proof. It remains to show that $J \cdot \mathcal{O}_{J, \mathcal{E}}^{0} \subseteq \mathcal{O}_{J, \mathcal{E}}^{0} \cdot I_{\mathcal{O}_{J, \mathcal{E}}^{1}}=I_{\mathcal{O}_{J, \mathcal{E}}^{1}}$. Since $\mathcal{O}_{J, \mathcal{E}}^{1}$ is a Hilbert bimodule, Katsura's ideal is equal to the range ideal of the left inner product, that is, the closed linear span of $x y^{*}$ for all $x, y \in \mathcal{O}_{J, \mathcal{E}}^{1}$. This contains $\mathbb{K}(\mathcal{E})$ for $x, y \in \mathcal{E}$, which in turn contains $J$ by the Cuntz-Pimsner covariance condition on $J$, see Proposition 3.1.17 So $J \cdot \mathcal{O}_{J, \mathcal{E}}^{0} \subseteq I_{\mathcal{O}_{J, \mathcal{E}}^{1}}$.

The relative Cuntz-Pimsner algebra of $\left(\mathcal{O}_{J, \mathcal{E}}^{0}, \mathcal{O}_{J, \mathcal{E}}^{1}, I_{\mathcal{O}_{J, \mathcal{E}}^{1}}\right)$ is again $\mathcal{O}_{J, \mathcal{E}}$ by Proposition 3.1.25 The correspondence $\mathcal{O}_{J, \mathcal{E}} \leadsto \mathcal{O}_{J, \mathcal{E}}$ associated to the covariant correspondence above is just the identity correspondence on $\mathcal{O}_{J, \mathcal{E}}$.

Remark 3.1.31. If $J_{A}=0$ or $J_{B}=\varphi^{-1}(\mathbb{K}(\mathcal{G}))$, then the condition $J_{A} \cdot \mathcal{F} \subseteq \mathcal{F} \cdot J_{B}$ for covariant correspondences $\left(A, \mathcal{E}, J_{A}\right) \rightarrow\left(B, \mathcal{G}, J_{B}\right)$ always holds and so may be left out. This is clear if $J_{A}=0$. Let $J_{B}=\varphi^{-1}(\mathbb{K}(\mathcal{G}))$. Since $\mathcal{F}$ is proper, $J_{A}$ acts on $\mathcal{E} \otimes_{A} \mathcal{F} \cong \mathcal{F} \otimes_{B} \mathcal{G}$ by compact operators by Lemma 3.1.7 Again by Lemma 3.1.7 this implies $J_{A} \subseteq \mathbb{K}\left(\mathcal{F} \cdot J_{B}\right)$. Thus $J_{A} \cdot \mathcal{F} \subseteq \mathcal{F} \cdot J_{B}$.

Example 3.1.32. Covariant correspondences are related to the T-pairs used by Katsura [31] to describe the ideal structure of relative Cuntz-Pimsner algebras. For this, we specialise to covariant correspondences out of $(A, \mathcal{E}, J)$ where the underlying correspondence comes from a quotient map $A \rightarrow A / I$. That is, $\mathcal{F}=A / I: A \leadsto A / I$ for an ideal $I \triangleleft A$. When is this part of a covariant correspondence from $(A, \mathcal{E}, J)$ to $\left(A / I, \mathcal{E}^{\prime}, J^{\prime}\right)$ for some $\mathcal{E}^{\prime}, J^{\prime}$ ?

There are natural isomorphisms $\mathcal{E} \otimes_{A} \mathcal{F} \cong \mathcal{E} / \mathcal{E} I$ and $\mathcal{F} \otimes_{A / I} \mathcal{E}^{\prime} \cong \mathcal{E}^{\prime}$ as correspondences $A \leadsto A / I$. So the only possible choice for $\mathcal{E}^{\prime}$ is $\mathcal{E}^{\prime}:=\mathcal{E} / \mathcal{E} I$ with a left $A / I$-action which gives the canonical $A$-action when composed with the quotient map $A \rightarrow A / I$. Such a correspondence $\mathcal{E} / \mathcal{E} I: A / I \leadsto A / I$ exists if and only if $\mathcal{E}$ is positively invariant, that is, $I \mathcal{E} \subseteq \mathcal{E} I$. Assume this to be the case.

An ideal $J^{\prime} \triangleleft A / I$ is equivalent to an ideal $I^{\prime} \triangleleft A$ that contains $I$. For a covariant correspondence, we require $J \mathcal{F} \subseteq \mathcal{F} J^{\prime}$, which means that $J \subseteq I^{\prime}$. And in order for $\left(A / I, \mathcal{E}^{\prime}, J^{\prime}\right)$ to be an object of $\mathfrak{C}_{\mathrm{pr}}^{\mathbb{N}}$, the ideal $J^{\prime}$ or, equivalently, $I^{\prime}$, should act by compact operators on $\mathcal{E}^{\prime}:=\mathcal{E} / \mathcal{E} I$.

Then there is an isomorphism $\mathcal{E} \otimes_{A} \mathcal{F} \cong \mathcal{F} \otimes_{A} \mathcal{E}^{\prime}$. It is unique up to an automorphism of $\mathcal{E} / \mathcal{E} I$, that is, a unitary operator on $\mathcal{E} / \mathcal{E} I$ that also commutes with the left action of $A$ or $A / I$, but this shall not concern us. So we get a covariant correspondence in this case. This induces a correspondence from $\mathcal{O}_{J, \mathcal{E}}$ 
to $\mathcal{O}_{J^{\prime}, \mathcal{E}^{\prime}}$ by Proposition 3.1 .28 Actually, our covariant correspondence is a covariant homomorphism, and so the correspondence from Proposition 3.1 .28 comes from a $\mathbb{T}$-equivariant ${ }^{*}$-homomorphism, which turns out to be surjective. So a pair of ideals $\left(I, I^{\prime}\right)$ as above induces a $\mathbb{T}$-equivariant quotient or, equivalently, a $\mathbb{T}$-invariant ideal in $\mathcal{O}_{J, \mathcal{E}}$.

Sometimes different pairs $\left(I, I^{\prime}\right)$ produce the same quotient of $\mathcal{O}_{J, \mathcal{E}}$. If $I^{\prime} / I$ contains elements that act by 0 on $\mathbb{K}(\mathcal{E} / \mathcal{E} I)$, then the map $A / I \rightarrow \mathcal{O}_{J^{\prime}, \mathcal{E}^{\prime}}$ is not injective by Proposition 3.1.13. Then we may enlarge $I$ without changing the relative Cuntz-Pimsner algebra. When we add the condition that no non-zero element of $I^{\prime} / I$ acts by a compact operator on $\mathcal{E} / \mathcal{E} \cdot I$, then we get exactly the $T$-pairs with $J \subseteq I^{\prime}$ of 31 . The $T$-pairs $\left(I, I^{\prime}\right)$ with $J \subseteq I^{\prime}$ correspond bijectively to gauge-invariant ideals of $\mathcal{O}_{J, \mathcal{E}}$ by [31, Proposition 11.9].

\subsection{Bicategories of correspondences and Hilbert bimodules}

We are going to enrich the relative Cuntz-Pimsner algebra construction to a homomorphism, or "functor" from a suitable bicategory of covariant correspondences to the $\mathbb{T}$-equivariant correspondence bicategory. Most of the work is already done in Proposition 3.1.28, which describes how this homomorphism acts on arrows. It remains to define the appropriate bicategories and write down the remaining data of a homomorphism.

The correspondence bicategory of $\mathrm{C}^{*}$-algebras and related bicategories have been discussed in 6 . 12 14. We recall basic bicategorical definitions in Appendix B Here we go through these notions much more quickly. Let $\mathfrak{C}$ be the correspondence bicategory. It has $\mathrm{C}^{*}$-algebras as objects, $\mathrm{C}^{*}$-correspondences as arrows, and correspondence isomorphisms as 2-arrows. The composition is the tensor product $\otimes_{B}$ of $\mathrm{C}^{*}$-correspondences.

Given any bicategory $\mathfrak{D}$, there is a bicategory $\mathfrak{C}^{\mathfrak{D}}$ with homomorphisms $\mathfrak{D} \rightarrow \mathfrak{C}$ as objects, transformations between these homomorphisms as arrows, and modifications between these transformations as 2-arrows (see the appendix for these notions). There is also a continuous version of this for a locally compact, topological bicategory $\mathfrak{D}$. In particular, we shall use the $\mathbb{T}$-equivariant correspondence bicategory $\mathfrak{C}^{\mathbb{T}}$. Its objects are $\mathrm{C}^{*}$-algebras with a continuous $\mathbb{T}$-action. Its arrows are $\mathbb{T}$-equivariant $\mathrm{C}^{*}$-correspondences, and 2-arrows are $\mathbb{T}$-equivariant isomorphisms of $\mathrm{C}^{*}$-correspondences.

When $\mathfrak{D}$ is the monoid $(\mathbb{N},+)$, we may simplify the bicategory $\mathfrak{C}^{\mathfrak{D}}$, see $[6$. Section 5]. An object in it is equivalent to a $\mathrm{C}^{*}$-algebra $A$ with a self-correspondence $\mathcal{E}: A \leadsto A$. An arrow is equivalent to a covariant correspondence (without the condition $J_{A} \mathcal{F} \subseteq \mathcal{F} J_{B}$ ), and a 2 -arrow is equivalent to an isomorphism between two covariant correspondences. The bicategory $\mathfrak{C}_{\mathrm{pr}}^{\mathbb{N}}$ that we need is a variant of $\mathfrak{C}^{\mathbb{N}}$ where we add the ideal $J$ and allow only proper covariant correspondences as arrows.

Definition 3.2.1. The bicategory $\mathfrak{C}_{\mathrm{pr}}^{\mathbb{N}}$ has the following data (see Definition B.1.1):

- Objects are triples $(A, \mathcal{E}, J)$, where $A$ is a $\mathrm{C}^{*}$-algebra, $\mathcal{E}: A \leadsto A$ is a $\mathrm{C}^{*}$-correspondence, and $J \subseteq \varphi^{-1}(\mathbb{K}(\mathcal{E}))$ is an ideal.

- Arrows $(A, \mathcal{E}, J) \rightarrow\left(A_{1}, \mathcal{E}_{1}, J_{1}\right)$ are proper covariant correspondences $(\mathcal{F}, u)$ from $(A, \mathcal{E}, J)$ to $\left(A_{1}, \mathcal{E}_{1}, J_{1}\right)$, that is, $\mathcal{F}$ is a proper correspondence $A \sim A_{1}$ with $J \mathcal{F} \subseteq \mathcal{F} J_{1}$ and $u$ is a correspondence isomorphism $\mathcal{E} \otimes_{A} \mathcal{F} \Rightarrow \mathcal{F} \otimes_{A_{1}} \mathcal{E}_{1}$.

- 2-Arrows $\left(\mathcal{F}_{0}, u_{0}\right) \Rightarrow\left(\mathcal{F}_{1}, u_{1}\right)$ are isomorphisms of covariant correspondences, that is, correspondence isomorphisms $w: \mathcal{F}_{0} \Rightarrow \mathcal{F}_{1}$ for which the following diagram commutes:

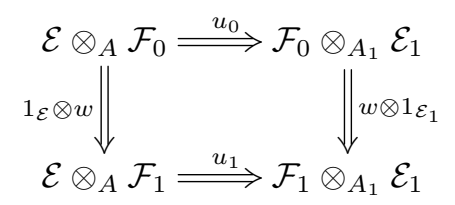

- The vertical product of 2-arrows

$$
w_{0}:\left(\mathcal{F}_{0}, u_{0}\right) \Rightarrow\left(\mathcal{F}_{1}, u_{1}\right), \quad w_{1}:\left(\mathcal{F}_{1}, u_{1}\right) \Rightarrow\left(\mathcal{F}_{2}, u_{2}\right)
$$

is the usual product $w_{1} \cdot w_{0}: \mathcal{F}_{0} \rightarrow \mathcal{F}_{2}$. This is indeed a 2 -arrow from $\left(\mathcal{F}_{0}, u_{0}\right)$ to $\left(\mathcal{F}_{2}, u_{2}\right)$. And the vertical product is associative and unital. Thus the arrows $(A, \mathcal{E}, J) \rightarrow\left(A_{1}, \mathcal{E}_{1}, J_{1}\right)$ and the 
2-arrows between them form a groupoid $\mathfrak{C}_{\mathrm{pr}}^{\mathbb{N}}\left((A, \mathcal{E}, J),\left(A_{1}, \mathcal{E}_{1}, J_{1}\right)\right)$.

- Let $(\mathcal{F}, u):(A, \mathcal{E}, J) \rightarrow\left(A_{1}, \mathcal{E}_{1}, J_{1}\right)$ and $\left(\mathcal{F}_{1}, u_{1}\right):\left(A_{1}, \mathcal{E}_{1}, J_{1}\right) \rightarrow\left(A_{2}, \mathcal{E}_{2}, J_{2}\right)$ be arrows. Their product is $\left(\mathcal{F}_{1}, u_{1}\right) \circ(\mathcal{F}, u):=\left(\mathcal{F} \otimes_{A_{1}} \mathcal{F}_{1}, u \bullet u_{1}\right)$, where $u \bullet u_{1}$ is the composite correspondence isomorphism

$$
\mathcal{E} \otimes_{A} \mathcal{F} \otimes_{A_{1}} \mathcal{F}_{1} \stackrel{u \otimes 1_{\mathcal{F}_{1}}}{\Longrightarrow} \mathcal{F} \otimes_{A_{1}} \mathcal{E}_{1} \otimes_{A_{1}} \mathcal{F}_{1} \stackrel{1_{\mathcal{F}} \otimes u_{1}}{\Longrightarrow} \mathcal{F} \otimes_{A_{1}} \mathcal{F}_{1} \otimes_{A_{2}} \mathcal{E}_{2}
$$

- The horizontal product for a diagram of arrows and 2-arrows

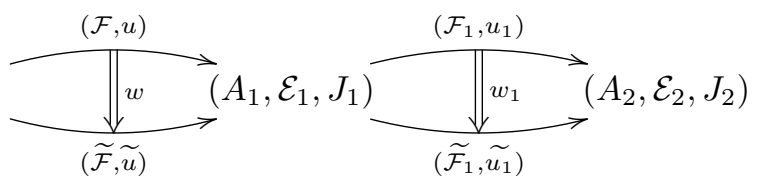

is the 2-arrow

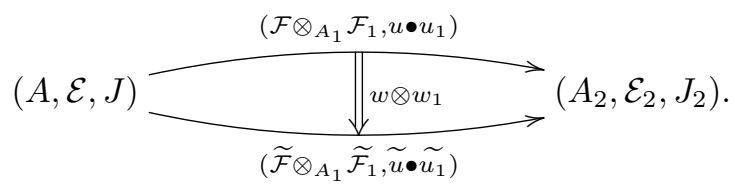

This horizontal product and the product of arrows combine to composition bifunctors

$$
\mathfrak{C}_{\mathrm{pr}}^{\mathbb{N}}\left((A, \mathcal{E}, J),\left(A_{1}, \mathcal{E}_{1}, J_{1}\right)\right) \times \mathfrak{C}_{\mathrm{pr}}^{\mathbb{N}}\left(\left(A_{1}, \mathcal{E}_{1}, J_{1}\right),\left(A_{2}, \mathcal{E}_{2}, J_{2}\right)\right) \rightarrow \mathfrak{C}_{\mathrm{pr}}^{\mathbb{N}}\left((A, \mathcal{E}, J),\left(A_{2}, \mathcal{E}_{2}, J_{2}\right)\right)
$$

- The unit arrow on the object $(A, \mathcal{E}, J)$ is the proper covariant correspondence $\left(A, \iota_{\mathcal{E}}\right)$, where $A$ is the identity correspondence, that is, $A$ with the obvious $A$-bimodule structure and the inner product $\langle x \mid y\rangle:=x^{*} y$, and $\iota_{\mathcal{E}}$ is the canonical isomorphism

$$
\mathcal{E} \otimes_{A} A \cong \mathcal{E} \cong A \otimes_{A} \mathcal{E}
$$

built from the right and left actions of $A$ on $\mathcal{E}$.

- The associators and unitors are the same as in the correspondence bicategory. Thus they inherit the coherence conditions needed for a bicategory.

Theorem 3.2.2. There is a homomorphism $\mathfrak{C}_{\mathrm{pr}}^{\mathbb{N}} \rightarrow \mathfrak{C}^{\mathbb{T}}$ that maps each object $(A, \mathcal{E}, J)$ to its relative Cunt-Pimsner algebra and is the construction of Proposition 3.1 .28 on arrows.

Proof. The construction in Proposition 3.1.28 is "natural" and thus functorial for isomorphisms of covariant correspondences, and it maps the identity covariant correspondence to the identity $\mathbb{T}$-equivariant correspondence on the relative Cuntz-Pimsner algebras. Let $(\mathcal{F}, u):(A, \mathcal{E}, J) \rightarrow$ $\left(A_{1}, \mathcal{E}_{1}, J_{1}\right)$ and $\left(\mathcal{F}_{1}, u_{1}\right):\left(A_{1}, \mathcal{E}_{1}, J_{1}\right) \rightarrow\left(A_{2}, \mathcal{E}_{2}, J_{2}\right)$ be covariant correspondences and let $\mathcal{O}_{\mathcal{F}, u}$ and $\mathcal{O}_{\mathcal{F}_{1}, u_{1}}$ be the associated $\mathbb{T}$-equivariant correspondences of relative Cuntz-Pimsner algebras. By definition, $\mathcal{O}_{\mathcal{F}, u} \otimes_{\mathcal{O}_{J_{1}, \mathcal{E}_{1}}} \mathcal{O}_{\mathcal{F}_{1}, u_{1}}$ and $\mathcal{O}_{\mathcal{F} \otimes_{A_{1}} \mathcal{F}_{1}, u \bullet u_{1}}$ are equal to $\left(\mathcal{F} \otimes_{A_{1}} \mathcal{O}_{J_{1}, \mathcal{F}_{1}}\right) \otimes_{\mathcal{O}_{J_{1}, \mathcal{F}_{1}}}\left(\mathcal{F}_{1} \otimes_{A_{2}} \mathcal{O}_{J_{2}, \mathcal{F}_{2}}\right)$ and $\left(\mathcal{F} \otimes_{A_{1}} \mathcal{F}_{1}\right) \otimes_{A_{2}} \mathcal{O}_{J_{2}, \mathcal{F}_{2}}$ as $\mathbb{T}$-equivariant correspondences $A \leadsto \mathcal{O}_{J_{2}, \mathcal{F}_{2}}$. Associators and unit transformations give a canonical $\mathbb{T}$-equivariant isomorphism between these correspondences. This isomorphism also intertwines the representations of $\mathcal{E}$. Hence it is a $\mathbb{T}$-equivariant isomorphism of correspondences $\mathcal{O}_{J, \mathcal{F}} \leadsto \mathcal{O}_{J_{2}, \mathcal{F}_{2}}$. These canonical isomorphisms satisfy the coherence conditions for a homomorphism of bicategories in Definition B.1.5.

The relative Cuntz-Pimsner algebra $\mathcal{O}_{J, \mathcal{E}}$ is the crossed product $\mathcal{O}_{J, \mathcal{E}}^{0} \rtimes \mathcal{O}_{J, \mathcal{E}}^{1}$ by Theorem 3.1 .24 So $\mathcal{O}_{J, \mathcal{E}}$ with the gauge $\mathbb{T}$-action and the Hilbert $\mathcal{O}_{J, \mathcal{E}}^{0}$-bimodule $\mathcal{O}_{J, \mathcal{E}}^{1}$ contain the same amount of information. We now study the construction that sends $(A, \mathcal{E}, J)$ to the Hilbert $\mathcal{O}_{J, \mathcal{E}^{-b i m o d u l e ~}}^{0} \mathcal{O}_{J, \mathcal{E}}^{1}$. The appropriate bicategory of Hilbert bimodules is a sub-bicategory of $\mathfrak{C}_{\mathrm{pr}}^{\mathbb{N}}$ :

Definition 3.2.3. Let $\mathfrak{C}_{\mathrm{pr}, *}^{\mathbb{N}} \subseteq \mathfrak{C}_{\mathrm{pr}}^{\mathbb{N}}$ be the full sub-bicategory whose objects are triples $\left(B, \mathcal{G}, I_{\mathcal{G}}\right)$, where $\mathcal{G}$ is a Hilbert $B$-bimodule and $I_{\mathcal{G}}$ is Katsura's ideal for $\mathcal{G}$, which is also equal to the range ideal $\langle\langle\mathcal{G} \mid \mathcal{G}\rangle\rangle$ of the left inner product on $\mathcal{G}$. The arrows and 2-arrows among objects of $\mathfrak{C}_{\mathrm{pr}, *}^{\mathbb{N}}$ are the same as in $\mathfrak{C}_{\text {pr }}^{\mathbb{N}}$, including the condition $I_{\mathcal{E}} \mathcal{F} \subseteq \mathcal{F} I_{\mathcal{G}}$ for covariant correspondences. 


\section{A BICATEGORICAL INTERPRETATION FOR RELATIVE CUNTZ-PIMSNER ALGEBRAS}

When we restrict the relative Cuntz-Pimsner algebra construction $\mathfrak{C}_{\mathrm{pr}}^{\mathbb{N}} \rightarrow \mathfrak{C}^{\mathbb{T}}$ to $\mathfrak{C}_{\mathrm{pr}, *}^{\mathbb{N}}$, we get the (partial) crossed product construction for Hilbert bimodules by Proposition 3.1.26. Thus Theorem 3.2.2 also completes the crossed product for Hilbert bimodules to a functor $\mathfrak{C}_{\mathrm{pr}, *}^{\mathbb{N}} \rightarrow \mathfrak{C}^{\mathbb{T}}$.

The map that sends $(A, \mathcal{E}, J)$ to $\left(\mathcal{O}_{J, \mathcal{E}}^{0}, \mathcal{O}_{J, \mathcal{E}}^{1}, I_{\mathcal{O}_{J \mathcal{E}}^{1}}\right)$ is part of a functor $\mathfrak{C}_{\mathrm{pr}}^{\mathbb{N}} \rightarrow \mathfrak{C}_{\mathrm{pr}, *}^{\mathbb{N}}$ which, when composed with the crossed product functor $\mathfrak{C}_{\mathrm{pr}, *}^{\mathbb{N}} \rightarrow \mathfrak{C}^{\mathbb{T}}$, gives the relative Cuntz-Pimsner algebra functor of Theorem 3.2 .2 We do not prove this now because it follows from our main result below. The key step is the following universal property of $\left(\mathcal{O}_{J, \mathcal{E}}^{0}, \mathcal{O}_{J, \mathcal{E}}^{1}, I_{\mathcal{O}_{J, \mathcal{E}}^{1}}\right)$ :

Proposition 3.2.4. Let $(A, \mathcal{E}, J)$ and $\left(B, \mathcal{G}, I_{\mathcal{G}}\right)$ be objects of $\mathfrak{C}_{\mathrm{pr}}^{\mathbb{N}}$ and $\mathfrak{C}_{\mathrm{pr}, *}^{\mathbb{N}}$, respectively. Let

$$
v_{(A, \mathcal{E}, J)}:(A, \mathcal{E}, J) \rightarrow\left(\mathcal{O}_{J, \mathcal{E}}^{0}, \mathcal{O}_{J, \mathcal{E}}^{1}, I_{\mathcal{O}_{J, \mathcal{E}}^{1}}\right)
$$

be the covariant correspondence from Lemma 3.1.30. Composition with $v_{(A, \mathcal{E}, J)}$ induces a groupoid equivalence

$$
\mathfrak{C}_{\mathrm{pr}}^{\mathbb{N}}\left((A, \mathcal{E}, J),\left(B, \mathcal{G}, I_{\mathcal{G}}\right)\right) \simeq \mathfrak{C}_{\mathrm{pr}, *}^{\mathbb{N}}\left(\left(\mathcal{O}_{J, \mathcal{E}}^{0}, \mathcal{O}_{J, \mathcal{E}}^{1}, I_{\mathcal{O}_{J, \mathcal{E}}^{1}}\right),\left(B, \mathcal{G}, I_{\mathcal{G}}\right)\right)
$$

Recall that $\mathfrak{C}_{\mathrm{pr}}^{\mathbb{N}}\left((A, \mathcal{E}, J),\left(A_{1}, \mathcal{E}_{1}, J_{1}\right)\right)$ for objects $(A, \mathcal{E}, J)$ and $\left(A_{1}, \mathcal{E}_{1}, J_{1}\right)$ of $\mathfrak{C}_{\mathrm{pr}}^{\mathbb{N}}$ denotes the groupoid with arrows $(A, \mathcal{E}, J) \rightarrow\left(A_{1}, \mathcal{E}_{1}, J_{1}\right)$ as objects and 2-arrows among them as arrows.

Proof. We begin with an auxiliary construction. Proposition 3.1.26 identifies $\mathcal{O}_{I_{\mathcal{G}}, \mathcal{G}} \cong B \rtimes \mathcal{G}$ as $\mathbb{Z}$-graded $\mathrm{C}^{*}$-algebras. In particular, $\mathcal{O}_{I_{\mathcal{G}}, \mathcal{G}}^{0} \cong B$, and $\mathcal{O}_{I_{\mathcal{G}}, \mathcal{G}}^{1} \cong \mathcal{G}$ and $\mathcal{O}_{I_{\mathcal{C}, \mathcal{G}}}^{-1} \cong \mathcal{G}^{*}$ as Hilbert $B$-bimodules. Let $(\mathcal{F}, u)$ be a proper covariant correspondence $(A, \mathcal{E}, J) \rightarrow\left(B, \mathcal{G}, I_{\mathcal{G}}\right)$. It induces a proper, $\mathbb{T}$-equivariant correspondence $\mathcal{O}_{\mathcal{F}, V}=\bigoplus_{n \in \mathbb{Z}} \mathcal{O}_{\mathcal{F}, V}^{n}$ from $\mathcal{O}_{J, \mathcal{E}}$ to $\mathcal{O}_{I_{\mathcal{G}}, \mathcal{G}}$ by Proposition 3.1.28 By construction, $\mathcal{O}_{\mathcal{F}, V}^{n}=\mathcal{F} \otimes_{B} \mathcal{O}_{I_{\mathcal{G}, \mathcal{G}}}^{n}$. Thus $\mathcal{O}_{\mathcal{F}, V}^{0}=\mathcal{F} \otimes_{B} \mathcal{O}_{I_{\mathcal{G}}, \mathcal{G}}^{0} \cong \mathcal{F} \otimes_{B} B \cong \mathcal{F}$ and $\mathcal{O}_{\mathcal{F}, V}^{1}=\mathcal{F} \otimes_{B} \mathcal{O}_{I_{\mathcal{G}}, \mathcal{G}}^{1} \cong \mathcal{F} \otimes_{B} \mathcal{G}$. The left action on $\mathcal{O}_{\mathcal{F}, V}$ is a nondegenerate, $\mathbb{T}$-equivariant ${ }^{*}$-homomorphism $\mathcal{O}_{J, \mathcal{E}} \rightarrow \mathbb{K}\left(\mathcal{O}_{\mathcal{F}, V}\right)$. So $\mathcal{O}_{J, \mathcal{E}}^{0}$ acts on $\mathcal{O}_{\mathcal{F}, V}$ by grading-preserving operators. Restricting to the degree-0 part, we get a nondegenerate *-homomorphism $\mathcal{O}_{J, \mathcal{E}}^{0} \rightarrow \mathbb{K}\left(\mathcal{O}_{\mathcal{F}, V}^{0}\right) \cong \mathbb{K}(\mathcal{F})$. Let $\mathcal{F} \#$ be $\mathcal{F}$ viewed as a correspondence $\mathcal{O}_{J, \mathcal{E}}^{0} \leadsto B$ in this way.

We now construct an isomorphism of correspondences

$$
u^{\#}: \mathcal{O}_{J, \mathcal{E}}^{1} \otimes_{\mathcal{O}_{J, \mathcal{E}}^{0}} \mathcal{F}^{\#} \Rightarrow \mathcal{F}^{\#} \otimes_{B} \mathcal{G} .
$$

We need two descriptions of $u^{\#}$. The first shows that it is unitary, the second that it intertwines the left actions of $\mathcal{O}_{J, \mathcal{E}}^{0}$. The first formula for $u^{\#}$ uses Lemma 3.1.23 which gives unitary Hilbert $B$-module maps

$$
\mathcal{O}_{J, \mathcal{E}}^{1} \otimes_{\mathcal{O}_{J, \mathcal{E}}^{0}} \mathcal{F}^{\#} \cong \mathcal{E} \otimes_{A} \mathcal{O}_{J, \mathcal{E}}^{0} \otimes_{\mathcal{O}_{J, \mathcal{E}}^{0}} \mathcal{F}^{\#} \cong \mathcal{E} \otimes_{A} \mathcal{F}
$$

Composing with $u: \mathcal{E} \otimes_{A} \mathcal{F} \Rightarrow \mathcal{F} \otimes_{B} \mathcal{G}$ gives the desired unitary $u^{\#}$. The second formula for $u^{\#}$ restricts the left action of $\mathcal{O}_{J, \mathcal{E}}$ on $\mathcal{O}_{\mathcal{F}, V}$ to a multiplication map

$$
\mathcal{O}_{J, \mathcal{E}}^{1} \otimes_{\mathcal{O}_{J, \mathcal{E}}^{0}} \mathcal{F}^{\#}=\mathcal{O}_{J, \mathcal{E}}^{1} \otimes_{\mathcal{O}_{J, \mathcal{E}}^{0}} \mathcal{O}_{\mathcal{F}, V}^{0} \rightarrow \mathcal{O}_{\mathcal{F}, V}^{1} \cong \mathcal{F}^{\#} \otimes_{B} \mathcal{G}
$$

This is manifestly $\mathcal{O}_{J, \mathcal{E}^{-}}^{0}$-linear because the isomorphism $\mathcal{F}^{\#} \otimes_{B} \mathcal{O}_{I_{\mathcal{G}}, \mathcal{G}}^{n} \cong \mathcal{O}_{\mathcal{F}, V}^{n}$ is by right multiplication and so intertwines the left actions of $\mathcal{O}_{J . \mathcal{E}}^{0}$. The map in (3.2.5) maps $t_{J}(\xi) \otimes \eta \mapsto u(\xi \otimes \eta)$ for all $\xi \in \mathcal{E}$, $\eta \in \mathcal{F}$. This determines it by Lemma 3.1 .23 So both constructions give the same map $u^{\#}$.

We claim that $I_{\mathcal{O}_{J, \mathcal{E}}^{1}} \cdot \mathcal{F}^{\#} \subseteq \mathcal{F}^{\#} \cdot I_{\mathcal{G}}$ holds, so that the pair $\left(\mathcal{F}^{\#}, u^{\#}\right)$ is a proper covariant correspondence from $\left(\mathcal{O}_{J, \mathcal{E}}^{0}, \mathcal{O}_{J, \mathcal{E}}^{1}, I_{\mathcal{O}_{J, \mathcal{E}}^{1}}\right)$ to $\left(B, \mathcal{G}, I_{\mathcal{G}}\right)$. The ideal $I_{\mathcal{O}_{J, \mathcal{E}}^{1}}$ is equal to the range of the left inner product on $\mathcal{O}_{J, \mathcal{E}}^{1}$. Using the Fell bundle structure, we may rewrite this as $\mathcal{O}_{J, \mathcal{E}}^{1} \cdot \mathcal{O}_{J, \mathcal{E}}^{-1}$. Thus

$$
I_{\mathcal{O}_{J, \mathcal{E}}^{1}} \cdot \mathcal{O}_{\mathcal{F}, V}^{0}=\mathcal{O}_{J, \mathcal{E}}^{1} \cdot \mathcal{O}_{J, \mathcal{E}}^{-1} \cdot \mathcal{O}_{\mathcal{F}, V}^{0} \subseteq \mathcal{O}_{J, \mathcal{E}}^{1} \cdot \mathcal{O}_{\mathcal{F}, V}^{-1}=\mathcal{E} \cdot \mathcal{O}_{J, \mathcal{E}}^{0} \cdot \mathcal{O}_{\mathcal{F}, V}^{-1}=\mathcal{E} \cdot \mathcal{O}_{\mathcal{F}, V}^{-1}
$$

The product $\mathcal{E} \cdot \mathcal{O}_{\mathcal{F}, V}^{-1}$ uses the representation of $\mathcal{E}$ on $\mathcal{O}_{\mathcal{F}, V}$ built in the proof of Proposition 3.1.28 So $\mathcal{E} \cdot \mathcal{O}_{\mathcal{F}, V}^{-1}$ is the image of the map

$$
\mathcal{E} \otimes_{A} \mathcal{F} \otimes_{B} \mathcal{G}^{*} \cong \mathcal{F} \otimes_{B} \mathcal{G} \otimes_{B} \mathcal{G}^{*}=\mathcal{F} \cdot I_{\mathcal{G}} .
$$

So $I_{\mathcal{O}_{J, \mathcal{E}}^{1}} \cdot \mathcal{O}_{\mathcal{F}, V}^{0} \subseteq \mathcal{F} \cdot I_{\mathcal{G}}$ as claimed. We have turned a proper covariant correspondence $(\mathcal{F}, u)$ from 
$(A, \mathcal{E}, J)$ to $\left(B, \mathcal{G}, I_{\mathcal{G}}\right)$ into a proper covariant correspondence $\left(\mathcal{F}^{\#}, u^{\#}\right)$ from $\left(\mathcal{O}_{J, \mathcal{E}}^{0}, \mathcal{O}_{J, \mathcal{E}}^{1}, I_{\mathcal{O}_{J, \mathcal{E}}^{1}}\right)$ to $\left(B, \mathcal{G}, I_{\mathcal{G}}\right)$.

Conversely, take a proper covariant correspondence $(\mathcal{F}, u)$ from $\left(\mathcal{O}_{J, \mathcal{E}}^{0}, \mathcal{O}_{J, \mathcal{E}}^{1}, I_{\mathcal{O}_{J, \mathcal{E}}^{1}}\right)$ to $\left(B, \mathcal{G}, I_{\mathcal{G}}\right)$. Composing it with $v_{(A, \mathcal{E}, J)}$ gives a proper covariant correspondence from $(A, \mathcal{E}, J)$ to $\left(B, \mathcal{G}, I_{\mathcal{G}}\right)$. We now simplify this product of covariant correspondences. The underlying correspondence $A \rightarrow \mathcal{O}_{J, \mathcal{E}}^{0}$ in $v_{(A, \mathcal{E}, J)}$ is $\mathcal{O}_{J, \mathcal{E}}^{0}$, and the isomorphism $\mathcal{E} \otimes_{A} \mathcal{O}_{J, \mathcal{E}}^{0} \cong \mathcal{O}_{J, \mathcal{E}}^{0} \otimes_{\mathcal{O}_{J, \mathcal{E}}^{0}} \mathcal{O}_{J, \mathcal{E}}^{1}=\mathcal{O}_{J, \mathcal{E}}^{1}$ is the one from Lemma 3.1.23 We identify the tensor product $\mathcal{O}_{J, \mathcal{E}}^{0} \otimes_{\mathcal{O}_{J, \mathcal{E}}^{0}} \mathcal{F}$ with $\mathcal{F}$ by the canonical map. Thus the product of $(\mathcal{F}, u)$ with $v_{(A, \mathcal{E}, J)}$ is canonically isomorphic to a covariant correspondence $\left(\mathcal{F}^{b}, u^{b}\right)$ with underlying correspondence $\mathcal{F}^{b}=\mathcal{F}: A \leadsto B$ with the left $A$-action through $\pi_{J}: A \rightarrow \mathcal{O}_{J, \mathcal{E}}^{0}$. The isomorphism $u^{b}: \mathcal{E} \otimes_{A} \mathcal{F}^{b} \Rightarrow \mathcal{F}^{b} \otimes_{B} \mathcal{G}$ is the composite of the given isomorphism $u: \mathcal{O}_{J, \mathcal{E}}^{1} \otimes_{\mathcal{O}_{J, \mathcal{E}}^{u}} \mathcal{F} \Rightarrow \mathcal{F} \otimes_{B} \mathcal{G}$ with the isomorphism $\mathcal{E} \otimes_{A} \mathcal{O}_{J, \mathcal{E}}^{0} \cong \mathcal{O}_{J, \mathcal{E}}^{1}$ from Lemma 3.1 .23

Now let $(\mathcal{F}, u)$ be a proper covariant correspondence from $(A, \mathcal{E}, J)$ to $\left(B, \mathcal{G}, I_{\mathcal{G}}\right)$. We claim that

$$
\left(\mathcal{F}^{\# b}, u^{\# b}\right)=(\mathcal{F}, u)
$$

By construction, the underlying Hilbert $B$-module of $\mathcal{F}^{\# b}$ is $\mathcal{F}$. We even have $\mathcal{F}^{\# b}=\mathcal{F}$ as correspondences $A \leadsto B$, that is, the left $\mathcal{O}_{J, \mathcal{E}}^{0}$-action on $\mathcal{F}^{\#}$ composed with $\pi_{J}: A \rightarrow \mathcal{O}_{J, \mathcal{E}}^{0}$ is the original action of $A$. The isomorphism $\mathcal{E} \otimes_{A} \mathcal{O}_{J, \mathcal{E}}^{0} \cong \mathcal{O}_{J, \mathcal{E}}^{1}$ is used both to get $u^{\#}$ from $u$ and to get $u^{\# b}$ from $u^{\#}$. Unravelling this shows that $u^{\# b}=u$.

Now we claim that the map that sends a proper covariant correspondence

$$
(\mathcal{F}, u):\left(\mathcal{O}_{J, \mathcal{E}}^{0}, \mathcal{O}_{J, \mathcal{E}}^{1}, I_{\mathcal{O}_{J, \mathcal{E}}^{1}}\right) \rightarrow\left(B, \mathcal{G}, I_{\mathcal{G}}\right)
$$

to $\left(\mathcal{F}^{b}, u^{b}\right)$ is injective. This claim and $(3.2 .6)$ imply $\left(\mathcal{F}^{b \#}, u^{b \#}\right)=(\mathcal{F}, u)$, that is, our two operations are inverse to each other. To prove injectivity, we use Proposition 3.1 .28 to build a correspondence $\mathcal{O}_{\mathcal{F}, u}: \mathcal{O}_{J, \mathcal{E}} \leadsto \mathcal{O}_{I_{\mathcal{G}}, \mathcal{G}}$ from $(\mathcal{F}, u)$. This correspondence determines $(\mathcal{F}, u)$ : we can get back $\mathcal{F}$ as its degree-0 part because $\mathcal{O}_{I_{\mathcal{G}}, \mathcal{G}}=B \rtimes \mathcal{G}$, and because $u$ and the left $\mathcal{O}_{J, \mathcal{E}^{-}}^{0}$-module structure on $\mathcal{F}$ are both contained in the left $\mathcal{O}_{J, \mathcal{E}}$-module structure on $\mathcal{O}_{\mathcal{F}, u}$. An $\mathcal{O}_{J, \mathcal{E}}$-module structure on $\mathcal{O}_{\mathcal{F}, u}$ is already determined by a representation of $(A, \mathcal{E})$. Since $\mathcal{O}_{I_{\mathcal{G}}, \mathcal{G}}^{n}=\mathcal{O}_{I_{\mathcal{G}}, \mathcal{G}}^{0} \cdot \mathcal{O}_{I_{\mathcal{G}}, \mathcal{G}}^{n}$, this representation is determined by its restriction to $\mathcal{O}_{\mathcal{F}, u}^{0} \cong \mathcal{F}$. And $\left(\mathcal{F}^{b}, u^{b}\right)$ determines the representation of $(A, \mathcal{E})$ on $\mathcal{F}$. Thus $\left(\mathcal{F}^{b}, u^{b}\right)$ determines $(\mathcal{F}, u)$.

The constructions of $\left(\mathcal{F}^{\#}, u^{\#}\right)$ and $\left(\mathcal{F}^{b}, u^{b}\right)$ are clearly natural for isomorphisms of covariant correspondences. So they form an isomorphism of groupoids

$$
\mathfrak{C}_{\mathrm{pr}}^{\mathbb{N}}\left((A, \mathcal{E}, J),\left(B, \mathcal{G}, I_{\mathcal{G}}\right)\right) \cong \mathfrak{C}_{\mathrm{pr}, *}^{\mathbb{N}}\left(\left(\mathcal{O}_{J, \mathcal{E}}^{0}, \mathcal{O}_{J, \mathcal{E}}^{1}, I_{\mathcal{O}_{J, \mathcal{E}}^{1}}\right),(B, \mathcal{G})\right)
$$

One piece in this isomorphism is naturally equivalent to the functor that composes with $v_{(A, \mathcal{E}, J)}$. Hence this functor is an equivalence of groupoids, as asserted.

\subsection{The reflector from correspondences to Hilbert bimodules}

We now strengthen Proposition 3.2.4 using some general results on adjunctions of homomorphisms between bicategories. We first recall the related and better known results about ordinary categories and functors.

Let $\mathcal{C}$ and $\mathcal{B}$ be categories. Let $R: \mathcal{C} \rightarrow \mathcal{B}$ be a functor and $b \in$ ob $\mathcal{B}$. An object $c \in$ ob $\mathcal{C}$ with an arrow $v: b \rightarrow R(c)$ is called a universal arrow from $b$ to $R$ if, for each $x \in$ ob $\mathcal{C}$ and each $f \in \mathcal{B}(b, R(x))$, there is a unique $g \in \mathcal{C}(c, x)$ with $R(g) \circ v=f$. Equivalently, the maps

$$
\mathcal{C}(c, x) \rightarrow \mathcal{B}(b, R(x)), \quad f \mapsto R(f) \circ v
$$

are bijective for all $x \in \mathrm{ob} \mathcal{C}$. The functor $R$ has a left adjoint $L: \mathcal{B} \rightarrow \mathcal{C}$ if and only if such universal arrows exist for all $x \in \mathrm{ob} \mathcal{C}$. The left adjoint functor $L: \mathcal{B} \rightarrow \mathcal{C}$ is uniquely determined up to natural isomorphism. It maps $b \mapsto c$ on objects, and the isomorphisms (3.3.1) become natural in both $b$ and $x$ when we replace $c$ by $L(b)$. An adjunction between $L$ and $R$ may also be expressed through its unit and counit, that is, natural transformations $L \circ R \Rightarrow \operatorname{id}_{\mathcal{C}}$ and $\operatorname{id}_{\mathcal{B}} \Rightarrow R \circ L$ such that the induced 


\section{A BICATEGORICAL INTERPRETATION FOR RELATIVE CUNTZ-PIMSNER ALGEBRAS}

transformations $L \Rightarrow L \circ R \circ L \Rightarrow L$ and $R \Rightarrow R \circ L \circ R \Rightarrow R$ are unit transformations.

A subcategory $\mathcal{C} \subseteq \mathcal{B}$ is called reflective if the inclusion functor $R: \mathcal{C} \rightarrow \mathcal{B}$ has a left adjoint $L: \mathcal{B} \rightarrow \mathcal{C}$. The functor $L$ is called reflector. The case we care about is a bicategorical version of a full subcategory. If $\mathcal{C} \subseteq \mathcal{B}$ is a full reflective subcategory, then we may choose $L \circ R$ to be the identity functor on $\mathcal{C}$ and the counit $L \circ R \Rightarrow \operatorname{id}_{\mathcal{C}}$ to be the unit natural transformation.

Fiore 24] carries the story of adjoint functors over to homomorphisms between 2-categories (which he calls "pseudo functors"), that is, bicategories where the associators and unitors are identity 2-arrows. The bicategories we need are not 2-categories. But any bicategory is equivalent to a 2-category by MacLane's Coherence Theorem. Hence Fiore's definitions and results apply in bicategories as well. We shorten notation by speaking of "universal" arrows and "adjunctions" instead of "biuniversal" arrows and "biadjunctions." A 2-category is also a category with some extra structure. So leaving out the prefix "bi" may cause confusion in that setting. But it will always be clear whether we mean the categorical or bicategorical notions.

Definition 3.3.2 (24, Definition 9.4]). Let $\mathcal{B}$ and $\mathcal{C}$ be bicategories, $R: \mathcal{C} \rightarrow \mathcal{B}$ a homomorphism, and $b \in$ ob $\mathcal{B}$. Let $c \in \mathrm{ob} \mathcal{C}$ and let $g: b \rightarrow R(c)$ be an arrow in $\mathcal{B}$. The pair $(c, g)$ is a universal arrow from $b$ to $R$ if, for every $x \in$ ob $\mathcal{C}$, the following functor is an equivalence of categories:

$$
g^{*}: \mathcal{C}(c, x) \rightarrow \mathcal{B}(b, R(x)), \quad f \mapsto R(f) \cdot g, \quad w \mapsto R(w) \bullet 1_{g} .
$$

Universal arrows are called left biliftings by Street 57 .

We can now reformulate Proposition 3.2.4.

Proposition 3.3.3. Let $(A, \mathcal{E}, J) \in \mathrm{ob} \mathfrak{C}_{\mathrm{pr}}^{\mathbb{N}}$. The covariant correspondence $v_{(A, \mathcal{E}, J)}$ from $(A, \mathcal{E}, J)$ to $\left(\mathcal{O}_{J, \mathcal{E}}^{0}, \mathcal{O}_{J, \mathcal{E}}^{1}, I_{\mathcal{O}_{J, \mathcal{E}}^{1}}\right)$ is a universal arrow from $(A, \mathcal{E}, J)$ to the inclusion homomorphism $\mathfrak{C}_{\mathrm{pr}, *}^{\mathbb{N}} \rightarrow \mathfrak{C}_{\mathrm{pr}}^{\mathbb{N}}$.

There are two alternative definitions of adjunctions, based on equivalences between morphism categories or on units and counits. These are spelled out, respectively, by Fiore in 24. Definition 9.8] and by Gurski in [28, Definition 2.1]. We shall use Fiore's definition.

Definition 3.3.4 ([24, Definition 9.8]). Let $\mathcal{B}$ and $\mathcal{C}$ be bicategories. An adjunction between them consists of

- two homomorphisms $L: \mathcal{B} \rightarrow \mathcal{C}, R: \mathcal{C} \rightarrow \mathcal{B}$;

- equivalences of categories

$$
\varphi_{b, c}: \mathcal{C}(L(b), c) \simeq \mathcal{B}(b, R(c))
$$

for all $b \in \mathrm{ob} \mathcal{B}, c \in \mathrm{ob} \mathcal{C}$;

- natural equivalences of functors

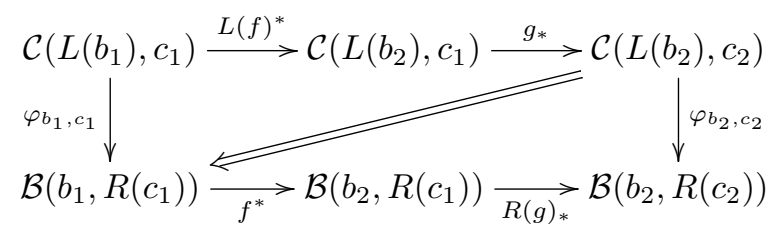

for all arrows $f: b_{2} \rightarrow b_{1}, g: c_{1} \rightarrow c_{2}$ in $\mathcal{B}$ and $\mathcal{C}$.

These are subject to a coherence condition. In brief, the functors $\varphi_{b, c}$ and the natural equivalences form a transformation between the homomorphisms

$$
\mathcal{B}^{\mathrm{op}} \times \mathcal{C} \rightrightarrows \text { Cat }, \quad(b, c) \mapsto \mathcal{C}(L(b), c), \mathcal{B}(b, R(c)) .
$$

Here Cat is the bicategory of categories, see Example B.1.2

Theorem 3.3.5 ([24, Theorem 9.17]). Let $\mathcal{B}$ and $\mathcal{C}$ be bicategories and let $R: \mathcal{C} \rightarrow \mathcal{B}$ be a homomorphism. It is part of an adjunction if and only if there are universal arrows from $c$ to $R$ for each object $c \in \mathrm{ob} \mathcal{C}$. 
More precisely, let $c_{b} \in \mathrm{ob} \mathcal{C}$ and $v_{b}: b \rightarrow R\left(c_{b}\right)$ for $b \in \mathrm{ob} \mathcal{C}$ be universal arrows from $b$ to $R$. Then there is an adjoint homomorphism $L: \mathcal{B} \rightarrow \mathcal{C}$ that maps $b \mapsto c_{b}$ on objects. In particular, this assignment is part of a homomorphism of bicategories.

Theorem 3.3.6 ([24, Theorem 9.20]). Two left adjoints $L, L^{\prime}: \mathcal{B} \rightrightarrows \mathcal{C}$ of $R: \mathcal{C} \rightarrow \mathcal{B}$ are equivalent, that is, there are transformations $L \Rightarrow L^{\prime}$ and $L^{\prime} \Rightarrow L$ that are inverse to each other up to invertible modifications.

Using these general theorems, we may strengthen Proposition 3.2 .4 (in the form of Proposition 3.3.3 to an adjunction theorem:

Corollary 3.3.7. The sub-bicategory $\mathfrak{C}_{\mathrm{pr}, *}^{\mathbb{N}} \subseteq \mathfrak{C}_{\mathrm{pr}}^{\mathbb{N}}$ is reflective, that is, the inclusion homomorphism $R: \mathfrak{C}_{\mathrm{pr}, *}^{\mathbb{N}} \rightarrow \mathfrak{C}_{\mathrm{pr}}^{\mathbb{N}}$ has a left adjoint (reflector) $L: \mathfrak{C}_{\mathrm{pr}}^{\mathbb{N}} \rightarrow \mathfrak{C}_{\mathrm{pr}, *}^{\mathbb{N}}$. On objects, this adjoint homomorphism maps

$$
(A, \mathcal{E}, J) \mapsto\left(\mathcal{O}_{J, \mathcal{E}}^{0}, \mathcal{O}_{J, \mathcal{E}}^{1}, I_{\mathcal{O}_{J, \mathcal{E}}^{1}}\right)
$$

The homomorphism $L$ is determined uniquely up to equivalence by Theorem 3.3 .6 . So we have characterised the construction of relative Cuntz-Pimsner algebras in bicategorical terms, as the reflector for the full sub-bicategory $\mathfrak{C}_{\mathrm{pr}, *}^{\mathbb{N}} \subseteq \mathfrak{C}_{\mathrm{pr}}^{\mathbb{N}}$. By Corollary 3.3.7, the relative Cuntz-Pimsner algebra construction is part of a homomorphism $L: \mathfrak{C}_{\mathrm{pr}}^{\mathbb{N}} \rightarrow \mathfrak{C}_{\mathrm{pr}, *}^{\mathbb{N}}$. For instance, this implies the following:

Corollary 3.3.8. The relative Cuntz-Pimsner algebras $\mathcal{O}_{J, \mathcal{E}}$ and $\mathcal{O}_{J_{1}, \mathcal{E}_{1}}$ are Morita equivalent if there is a Morita equivalence $\mathcal{F}$ between $\mathcal{E}$ and $\mathcal{E}_{1}$ as in 42 , Definition 2.1] with $J \cdot \mathcal{F}=\mathcal{F} \cdot J_{1}$.

Example 3.3.9. Let $\mathcal{E}: A \leadsto B$ be a correspondence. Let $\mathcal{F}: B \leadsto A$ be an imprimitivity $A, B$-bimodule. We let $\mathcal{G}_{A}=\mathcal{E} \otimes_{B} \mathcal{F}$ and $\mathcal{G}_{B}=\mathcal{F} \otimes_{A} \mathcal{E}$ be the associated correspondences over $A$ and $B$, respectively. Let $\mathcal{F}^{*}: A \leadsto B$ be the imprimitivity $B, A$-bimodule adjoint to $\mathcal{F}$. The canonical isomorphisms

$$
\mathcal{F}^{*} \otimes_{B} \mathcal{F} \cong A, \quad \mathcal{F} \otimes_{A} \mathcal{F}^{*} \cong B
$$

from Proposition 3.1 .6 yield a correspondence isomorphism

$$
\mathcal{G}_{A} \otimes_{A} \mathcal{F}^{*}=\mathcal{E} \otimes_{B} \mathcal{F} \otimes_{A} \mathcal{F}^{*} \cong \mathcal{E} \cong \mathcal{F}^{*} \otimes_{B} \mathcal{F} \otimes_{A} \mathcal{E}=\mathcal{F}^{*} \otimes_{B} \mathcal{G}_{B}
$$

It follows from Lemma 3.1 .7 that $b \in B$ is compact on $\mathcal{G}_{B}$ if and only if $b \mathcal{F} \subseteq \mathcal{F} \varphi^{-1}(\mathbb{K}(\mathcal{E}))$. So for each ideal $J_{A} \triangleleft A$ with $J_{A} \subseteq \varphi^{-1}(\mathbb{K}(\mathcal{E}))$, the ideal $J_{B}:=\left\langle\left\langle\mathcal{F} J_{A} \mid \mathcal{F}\right\rangle\right\rangle$ in $B$ acts by compact operators on $\mathcal{G}_{B}$. Similarly, for each ideal $J_{B}$ in $B$ acting by compact operators on $\mathcal{G}_{B}, J_{A}:=\left\langle J_{B} \mathcal{F} \mid \mathcal{F}\right\rangle$ is an ideal of $A$ that acts by compact operators on $\mathcal{E}$, and hence on $\mathcal{G}_{A}$. Therefore, the bijection between the lattices of ideals of $A$ and $B$, respectively, coming from the Rieffel correspondence (see Theorem A.2.11), provides a bijection between ideals in $A$ acting by compact operators on $\mathcal{G}_{A}$ and ideals of $B$ mapped to compact operators on $\mathcal{G}_{B}$. In particular, up to Morita equivalence, there is a bijection between relative Cuntz-Pimsner algebras associated to $\mathcal{G}_{A}$ and $\mathcal{G}_{B}$.

The proof of Theorem 3.3.5 also describes the adjoint functor. We now describe the reflector $L: \mathfrak{C}_{\mathrm{pr}}^{\mathbb{N}} \rightarrow \mathfrak{C}_{\mathrm{pr}, *}^{\mathbb{N}}$ explicitly, thereby explaining part of the proof of Theorem 3.3.5. Much of the work in this proof is needed to check that various diagrams of 2-arrows commute. We do not repeat these computations here.

The homomorphism $L$ maps $(A, \mathcal{E}, J) \mapsto\left(\mathcal{O}_{J, \mathcal{E}}^{0}, \mathcal{O}_{J, \mathcal{E}}^{1}, I_{\mathcal{O}_{J, \mathcal{E}}^{1}}\right)$ on objects. Let $(A, \mathcal{E}, J)$ and $\left(A_{1}, \mathcal{E}_{1}, J_{1}\right)$ be objects of $\mathfrak{C}_{\mathrm{pr}}^{\mathbb{N}}$ and let $(\mathcal{F}, u):(A, \mathcal{E}, J) \rightarrow\left(A_{1}, \mathcal{E}_{1}, J_{1}\right)$ be proper covariant correspondences. We use the notation of the proof of Proposition 3.2.4 and write $\bar{\iota}_{\mathcal{E}_{1}}$ for the canonical isomorphism $\mathcal{E}_{1} \otimes_{A_{1}} \mathcal{O}_{J_{1}, \mathcal{E}_{1}}^{0} \cong \mathcal{O}_{J_{1}, \mathcal{E}_{1}}^{1} \otimes_{\mathcal{O}_{J_{1}, \mathcal{E}_{1}}^{0}} \mathcal{O}_{J_{1}, \mathcal{E}_{1}}^{0}$ from Lemma 3.1.23, which is the covariance part of $v_{\left(A_{1}, \mathcal{E}_{1}, J_{1}\right)}$. Let

$$
\begin{aligned}
L(\mathcal{F}, u):\left(\mathcal{O}_{J, \mathcal{E}}^{0}, \mathcal{O}_{J, \mathcal{E}}^{1}, I_{\mathcal{O}_{J, \mathcal{E}}^{1}}\right) \rightarrow\left(\mathcal{O}_{J_{1}, \mathcal{E}_{1}}^{0}, \mathcal{O}_{J_{1}, \mathcal{E}_{1}}^{1}, I_{\mathcal{O}_{J_{1}, \mathcal{E}_{1}}^{1}}\right), \\
L(\mathcal{F}, u):=\left(\left(\mathcal{F} \otimes_{A_{1}} \mathcal{O}_{J_{1}, \mathcal{E}_{1}}^{0}\right)^{\#},\left(u \bullet \bar{\iota}_{\mathcal{E}_{1}}\right)^{\#}\right) .
\end{aligned}
$$

In other words, we first compose $(\mathcal{F}, u)$ with $v_{\left(A_{1}, \mathcal{E}_{1}, J_{1}\right)}$ to get a covariant correspondence $\left(\mathcal{F} \otimes_{A_{1}}\right.$ $\left.\mathcal{O}_{J_{1}, \mathcal{E}_{1}}^{0}, u \bullet \bar{\iota}_{\mathcal{E}_{1}}\right)$ from $(A, \mathcal{E}, J)$ to $\left(\mathcal{O}_{J_{1}, \mathcal{E}_{1}}^{0}, \mathcal{O}_{J_{1}, \mathcal{E}_{1}}^{1}, I_{\mathcal{O}_{J_{1}, \mathcal{E}_{1}}^{1}}^{1}\right)$ and then apply the equivalence in Proposition 3.2 .4 


\section{A BICATEGORICAL INTERPRETATION FOR RELATIVE CUNTZ-PIMSNER ALGEBRAS}

The construction on covariant correspondences above is clearly "natural", that is, functorial for isomorphisms. Explicitly, $L$ maps an isomorphism of covariant correspondences $w:(\mathcal{F}, u) \Rightarrow\left(\mathcal{F}^{\prime}, u^{\prime}\right)$ to

$$
L(w):=\left(w \otimes 1_{\mathcal{O}_{J_{1}, \mathcal{E}_{1}}^{0}}\right)^{\#}: L(\mathcal{F}, u) \Rightarrow L\left(\mathcal{F}^{\prime}, u^{\prime}\right)
$$

To make $L$ a homomorphism, we also need compatibility data for units and composition of arrows. The construction of $L$ above maps the identity covariant correspondence on $(A, \mathcal{E}, J)$ to $v_{(A, \mathcal{E}, J)}^{\#}:\left(\mathcal{O}_{J, \mathcal{E}}^{0}, \mathcal{O}_{J, \mathcal{E}}^{1}, I_{\mathcal{E}}\right) \rightarrow\left(\mathcal{O}_{J, \mathcal{E}}^{0}, \mathcal{O}_{J, \mathcal{E}}^{1}, I_{\mathcal{E}}\right)$. This is canonically isomorphic to the identity covariant correspondence on $\left(\mathcal{O}_{J, \mathcal{E}}^{0}, \mathcal{O}_{J, \mathcal{E}}^{1}, I_{\mathcal{E}}\right)$ because the equivalence in Proposition 3.2 .4 is by composition with $v_{(A, \mathcal{E}, J)}$. This is the unit part in our homomorphism $L$.

Let $(\mathcal{F}, u):(A, \mathcal{E}, J) \rightarrow\left(A_{1}, \mathcal{E}_{1}, J_{1}\right)$ and $\left(\mathcal{F}_{1}, u_{1}\right):\left(A_{1}, \mathcal{E}_{1}, J_{1}\right) \rightarrow\left(A_{2}, \mathcal{E}_{2}, J_{2}\right)$ be proper covariant correspondences. Then the homomorphism $L$ contains isomorphisms of covariant correspondences

$$
\lambda\left((\mathcal{F}, u),\left(\mathcal{F}_{1}, u_{1}\right)\right): L(\mathcal{F}, u) \circ L\left(\mathcal{F}_{1}, u_{1}\right) \Rightarrow L\left((\mathcal{F}, u) \circ\left(\mathcal{F}_{1}, u_{1}\right)\right),
$$

which are natural for isomorphisms of covariant correspondences and satisfy some coherence conditions when we compose three covariant correspondences or compose with identity covariant correspondences. We take $\lambda$ to be the isomorphism

$$
\left(\mathcal{F}_{0} \otimes_{A_{1}} \mathcal{O}_{J_{1}, \mathcal{E}_{1}}^{0}\right) \otimes_{\mathcal{O}_{J_{1}, \mathcal{E}_{1}}^{0}}\left(\mathcal{F}_{1} \otimes_{A_{2}} \mathcal{O}_{J_{2}, \mathcal{E}_{2}}^{0} \cong\left(\mathcal{F}_{0} \otimes_{A_{1}} \mathcal{F}_{1}\right) \otimes_{A_{2}} \mathcal{O}_{J_{2}, \mathcal{E}_{2}}^{0}\right.
$$

given by the left action of $\mathcal{O}_{J_{1}, \mathcal{E}_{1}}^{0}$ on $\mathcal{F}_{1} \otimes_{A_{2}} \mathcal{O}_{J_{2}, \mathcal{E}_{2}}^{0}$ that is constructed in the proof of Proposition 3.1 .28

The proof of Theorem 3.3 .5 builds $\lambda$ using only the universality of the arrows $v_{(A, \mathcal{E}, J)}$. By the equivalence of categories in Proposition 3.2.4 whiskering (horizontal composition) with $v_{(A, \mathcal{E}, J)}$ maps isomorphisms as in 3.3 .10 bijectively to isomorphisms

$$
v_{(A, \mathcal{E}, J)} \circ L(\mathcal{F}, u) \circ L\left(\mathcal{F}_{1}, u_{1}\right) \Rightarrow v_{(A, \mathcal{E}, J)} \circ L\left((\mathcal{F}, u) \circ\left(\mathcal{F}_{1}, u_{1}\right)\right) .
$$

The construction of $L$ implies $v_{(A, \mathcal{E}, J)} \circ L(\mathcal{F}, u) \circ L\left(\mathcal{F}_{1}, u_{1}\right) \cong(\mathcal{F}, u) \circ v_{\left(A_{1}, \mathcal{E}_{1}, J_{1}\right)} \circ L\left(\mathcal{F}_{1}, u_{1}\right) \cong(\mathcal{F}, u) \circ$ $\left(\mathcal{F}_{1}, u_{1}\right) \circ v_{\left(A_{2}, \mathcal{E}_{2}, J_{2}\right)}$ and $v_{(A, \mathcal{E}, J)} \circ L\left((\mathcal{F}, u) \circ\left(\mathcal{F}_{1}, u_{1}\right)\right) \cong\left((\mathcal{F}, u) \circ\left(\mathcal{F}_{1}, u_{1}\right)\right) \circ v_{\left(A_{2}, \mathcal{E}_{2}, J_{2}\right)}$, where we disregard associators. Hence there is a canonical isomorphism of covariant correspondences as in (3.3.11). This Ansatz produces the same isomorphisms $\lambda$ as above. We have now described the data of the homomorphism $L$. Fiore proves in [24] that it is indeed a homomorphism.

Proposition 3.3.12. The composite of $L$ and the crossed product homomorphism $\mathfrak{C}_{\mathrm{pr}, *}^{\mathbb{N}} \rightarrow \mathfrak{C}^{\mathbb{T}}$ is naturally isomorphic to the homomorphism $\mathfrak{C}_{\mathrm{pr}}^{\mathbb{N}} \rightarrow \mathfrak{C}^{\mathbb{T}}$ of Theorem 3.2 .2 .

Proof. Our homomorphisms agree on objects by Proposition 3.1.25. The proof of Proposition 3.2.4 constructed the covariant correspondence $\left(\mathcal{F}^{\#}, u^{\#}\right)$ by taking the degree-0 part in the correspondence constructed in the proof of Proposition 3.1.28 Thus we may build a natural isomorphism between the functors in question out of the nondegenerate left action of $\mathcal{O}_{J_{1}, \mathcal{E}_{1}}^{0}$ on $\mathcal{O}_{J_{1}, \mathcal{E}_{1}}$.

So the reflector $L$ lifts the Cuntz-Pimsner algebra homomorphism $\mathfrak{C}_{\mathrm{pr}}^{\mathbb{N}} \rightarrow \mathfrak{C}^{\mathbb{T}}$ to a homomorphism with values in $\mathfrak{C}_{\mathrm{pr}, *}^{\mathbb{N}}$. Such a lifting should exist because a Hilbert bimodule and its crossed product with the $\mathbb{T}$-action determine each other.

An adjunction also contains "natural" equivalences of categories $\varphi_{b, c}: \mathcal{C}(L(b), c) \simeq \mathcal{B}(b, R(c))$, where naturality is further data, see Definition 3.3.4 In the case at hand, these equivalences are exactly the equivalences of categories

$$
v_{(A, \mathcal{E}, J)}^{*}: \mathfrak{C}_{\mathrm{pr}}^{\mathbb{N}}\left((A, \mathcal{E}, J),\left(B, \mathcal{G}, I_{\mathcal{G}}\right)\right) \simeq \mathfrak{C}_{\mathrm{pr}, *}^{\mathbb{N}}\left(\left(\mathcal{O}_{J, \mathcal{E}}^{0}, \mathcal{O}_{J, \mathcal{E}}^{1}, I_{\mathcal{O}_{J, \mathcal{E}}^{1}}\right),\left(B, \mathcal{G}, I_{\mathcal{G}}\right)\right)
$$

in Proposition 3.2.4. Their naturality boils down to the canonical isomorphisms of correspondences $v_{(A, \mathcal{E}, J)} \circ L(\mathcal{F}, u) \cong(\mathcal{F}, u) \circ v_{(A, \mathcal{E}, J)}$, which we have already used above to describe the multiplicativity data $\lambda$ in the homomorphism $L$.

Finally, we relate our adjunction to the colimit description of Cuntz-Pimsner algebras in 6]. Let $\mathcal{C}$ and $\mathcal{D}$ be categories. Let $\mathcal{C}^{\mathcal{D}}$ be the category of functors $\mathcal{D} \rightarrow \mathcal{C}$, which are also called diagrams of shape $\mathcal{D}$ in $\mathcal{C}$. Identify $\mathcal{C}$ with the subcategory of "constant" diagrams in $\mathcal{C}^{\mathcal{D}}$. This subcategory is reflective if and only if all $\mathcal{D}$-shaped diagrams in $\mathcal{C}$ have a colimit, and the reflector maps a diagram to its colimit. 
This remains true for the bicategorical colimits in [6]: by definition, the colimit of a diagram is a universal arrow to a constant diagram. In our context, a constant diagram in $\mathfrak{C}_{\mathrm{pr}}^{\mathbb{N}}$ is an object of the form $(B, B, B)$ that is, the Hilbert $B$-bimodule is the identity bimodule and $J=B$ as always for objects of $\mathfrak{C}_{\mathrm{pr}, *}^{\mathbb{N}}$. Since the condition $J \cdot \mathcal{F} \subseteq \mathcal{F} \cdot B$ always holds, the ideal $J$ plays no role, compare Remark 3.1.31.

A proper covariant correspondence $(A, \mathcal{E}, J) \rightarrow(B, B, B)$ is equivalent to a proper correspondence $\mathcal{F}: A \leadsto B$ with an isomorphism $\mathcal{E} \otimes_{A} \mathcal{F} \Rightarrow \mathcal{F}$ because $\mathcal{F} \otimes_{B} B \cong \mathcal{F}$. As shown in [6], such a pair is equivalent to a representation $(\varphi, t)$ of the correspondence $\mathcal{E}$ on $\mathcal{F}$ that is nondegenerate in the sense that $t(\mathcal{E}) \cdot \mathcal{F}=\mathcal{F}$. The properness of $\mathcal{F}$ means that $\varphi(A) \subseteq \mathbb{K}(\mathcal{F})$, which implies $t(\mathcal{E}) \subseteq \mathbb{K}(\mathcal{F})$.

It is shown in [6] that all diagrams of proper correspondences of any shape have a colimit. This is probably false for diagrams of non-proper correspondences, such as the correspondence $\ell^{2}(\mathbb{N}): \mathbb{C} \sim \mathbb{C}$ that defines the Cuntz algebra $\mathcal{O}_{\infty}$. The way around this problem that we found here is to enlarge the sub-bicategory of constant diagrams, allowing diagrams of Hilbert bimodules. In addition, we added an ideal $J$ to have enough data to build relative Cuntz-Pimsner algebras.

Since the sub-bicategory $\mathfrak{C}^{\mathfrak{C}} \subseteq \mathfrak{C}_{\mathrm{pr}}^{\mathbb{N}}$ of constant diagrams is contained in $\mathfrak{C}_{\mathrm{pr}, *}^{\mathbb{N}}$, we may relate universal arrows to objects in $\mathfrak{C}^{\mathfrak{c}}$ and $\mathfrak{C}_{\mathrm{pr}, *}^{\mathbb{N}}$ as follows. Let $(A, \mathcal{E}, J)$ be an object of $\mathfrak{C}_{\mathrm{pr}}^{\mathbb{N}}$. Then $v_{(A, \mathcal{E}, J)}:(A, \mathcal{E}, J) \rightarrow\left(\mathcal{O}_{J, \mathcal{E}}^{0}, \mathcal{O}_{J, \mathcal{E}}^{1}, I_{\mathcal{O}_{J, \mathcal{E}}^{1}}\right)$ is a universal arrow to an object of $\mathfrak{C}_{\mathrm{pr}, *}^{\mathbb{N}}$ by Proposition 3.3 .3 The universality of $v_{(A, \mathcal{E}, J)}$ implies that a universal arrow from $(A, \mathcal{E}, J)$ to a constant diagram factors through $v_{(A, \mathcal{E}, J)}$, and that an arrow from $\left(\mathcal{O}_{J, \mathcal{E}}^{0}, \mathcal{O}_{J, \mathcal{E}}^{1}, I_{\mathcal{O}_{J, \mathcal{E}}^{1}}\right)$ to a constant diagram is universal if and only if its composite with $v_{(A, \mathcal{E}, J)}$ is universal. In other words, the diagram $(A, \mathcal{E}, J)$ has a colimit if and only if $\left(\mathcal{O}_{J, \mathcal{E}}^{0}, \mathcal{O}_{J, \mathcal{E}}^{1}, I_{\mathcal{O}_{J, \mathcal{E}}^{1}}\right)$ has one, and then the two colimits are the same. We are dealing with the same colimits as in 6 because the ideal $J$ in $(A, \mathcal{E}, J)$ plays no role for arrows to constant diagrams. 


\section{Chapter 4}

\section{Product systems and some $\mathrm{C}^{*}$-constructions}

In this chapter, we recall certain constructions of $\mathrm{C}^{*}$-algebras associated to product systems, such as Toeplitz algebras. We describe the spectral subspaces for the canonical coaction of a group containing the underlying semigroup. In Section 4.2 we restrict our attention to compactly aligned product systems over quasi-lattice ordered groups. We provide examples of $\mathrm{C}^{*}$-algebras associated to product systems coming from semigroups of endomorphisms. With the subsequent chapters in mind, we prove some facts concerning the canonical grading of relative Cuntz-Pimsner algebras. We finish this chapter by defining bicategories of compactly aligned product systems.

\subsection{Basic notions}

Our main reference for this section is the original paper by Fowler [26], in which he introduced the concept of product systems in this generality.

\subsubsection{Toeplitz algebras}

Let $P$ be a semigroup with identity $e$ and $A$ a $\mathrm{C}^{*}$-algebra. A product system over $P$ of $A$-correspondences consists of:

(i) a correspondence $\mathcal{E}_{p}: A \leadsto A$ for each $p \in P$;

(ii) correspondence isomorphisms $\mu_{p, q}: \mathcal{E}_{p} \otimes_{A} \mathcal{E}_{q} \cong \mathcal{E}_{p q}$, also called multiplication maps, for all $p, q \in P \backslash\{e\} ;$

We require that $\mathcal{E}_{e}=A$ be the canonical correspondence over $A$. Let $\varphi_{p}: A \rightarrow \mathbb{B}\left(\mathcal{E}_{p}\right)$ be the multiplication map $\mu_{e, p}$ and let $\mu_{p, e}$ implement the right action of $A$ on $\mathcal{E}_{p}$, respectively, so that $\mu_{e, p}(a \otimes$ $\left.\xi_{p}\right)=\varphi_{p}(a) \xi_{p}$ and $\mu_{p, e}\left(\xi_{p} \otimes a\right)=\xi_{p} a$ for all $a \in A$ and $\xi_{p} \in \mathcal{E}_{p}$.

This data must make the following diagram commute:

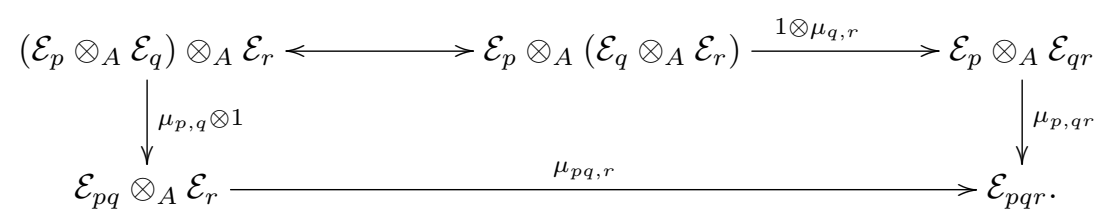

A product system $\mathcal{E}=\left(\mathcal{E}_{p}\right)_{p \in P}$ will be called faithful if $\varphi_{p}$ is injective for all $p \in P$. It is proper if $A$ acts by compact operators on $\mathcal{E}_{p}$ for all $p$ in $P$. If each $\mathcal{E}_{p}$ is a Hilbert $A$-bimodule, we will speak of a product system of Hilbert bimodules.

Example 4.1.1. Let $P$ be a semigroup. For each $p \in P$, set $\mathcal{E}_{p}:=\mathbb{C}$. This gives a product system over $P$ with multiplication and inner product inherited from the structure of $\mathrm{C}^{*}$-algebra of $\mathbb{C}$. 
Example 4.1.2. An endomorphism of a $\mathrm{C}^{*}$-algebra $A$ is said to be extendible if it extends to a strictly continuous endomorphism of the multiplier algebra $M(A)$ (see [4]). This happens if and only if there is a projection $Q \in M(A)$ so that $\alpha\left(u_{\lambda}\right)$ converges to $Q$ in the strict topology of $M(A)$, where $\left(u_{\lambda}\right)_{\lambda \in \Lambda}$ is an approximate unit for $A$. In particular, we have $Q a=\lim _{\lambda} \alpha\left(u_{\lambda}\right) a=\alpha(1) a$ for all $a \in A$. Let $\alpha: P \rightarrow$ $\operatorname{End}(A)$ be an action by extendible endomorphisms with $\alpha_{e}=\operatorname{id}_{A}{ }^{1}$ For each $p \in P$, let ${ }_{\alpha_{p}} A:=\alpha_{p}(1) A$ be equipped with the struture of right Hilbert $A$-module coming from the multiplication and involution operations on $A$. That is, $\alpha_{p}(1) a \cdot b:=\alpha_{p}(1) a b$ and $\left\langle\alpha_{p}(1) a \mid \alpha_{p}(1) b\right\rangle:=a^{*} \alpha_{p}(1) b$ for all $a, b \in A$. Here we still denote by $\alpha_{p}$ the corresponding endomorphism of $M(A)$. We let $\varphi_{p}: A \rightarrow \mathbb{B}\left({ }_{\alpha_{p}} A\right)$ be the ${ }^{*}$-homomorphism implemented by $\alpha_{p}$. So $\varphi_{p}(b)\left(\alpha_{p}(1) a\right)=\alpha_{p}(b) a$. This turns ${ }_{\alpha_{p}} A$ into a correspondence over $A$.

We let $\mu_{p, q}:{ }_{\alpha_{p}} A \otimes_{A}{ }_{\alpha_{q}} A \rightarrow{ }_{\alpha_{q p}} A$ be defined on elementary tensors by

$$
\alpha_{p}(1) a \otimes_{A} \alpha_{q}(1) b \mapsto \alpha_{q p}(1) \alpha_{q}(a) b .
$$

This intertwines the left and right actions of $A$ and preserves the $A$-valued inner product. It is surjective because

$$
\alpha_{q p}(1) a=\lim _{\lambda} \alpha_{q p}\left(u_{\lambda}\right) a=\lim _{\lambda} \alpha_{q}\left(\alpha_{p}\left(u_{\lambda}\right)\right) a=\lim _{\lambda} \alpha_{q}\left(\alpha_{p}(1) \alpha_{p}\left(u_{\lambda}\right)\right) a .
$$

Since $p \mapsto \alpha_{p}$ is an action by endomorphisms, the multiplication maps are associative. Thus, $\alpha: P \rightarrow$ $\operatorname{End}(A)$ gives rise to a product system ${ }_{\alpha} A=\left({ }_{\alpha_{p}} A\right)_{p \in P}$ over $P^{\text {op }}$, where $P^{\text {op }}$ is the opposite semigroup of $P$. Moreover, ${ }_{\alpha} A$ is proper, since $\mathbb{K}\left({ }_{\alpha_{p}} A\right) \cong \alpha_{p}(1) A \alpha_{p}(1)$ and $\alpha_{p}(a)=\alpha_{p}(1) \alpha_{p}(a) \alpha_{p}(1)$ for all $a \in A$ and $p \in P$.

Example 4.1.3. Let $\alpha: P \rightarrow \operatorname{End}(A)$ be an action by extendible endomorphisms as above with the additional property that, for all $p \in P, \alpha_{p}$ is an injective endomorphism with hereditary range. In this case, ${ }_{\alpha} A$ is faithful and may be enriched to a product system of Hilbert bimodules over $P^{\text {op }}$. The left $A$-valued inner product is given by

$$
\left\langle\left\langle\alpha_{p}(1) a \mid \alpha_{p}(1) b\right\rangle\right\rangle=\alpha_{p}^{-1}\left(\alpha_{p}(1) a b^{*} \alpha_{p}(1)\right)
$$

for all $a, b \in A$ and $p \in P$. In particular, this yields a product system $A_{\alpha}=\left(A_{\alpha_{p}}\right)_{p \in P}$ over $P$, where $A_{\alpha_{p}}=A \alpha_{p}(1)$ with the Hilbert $A$-bimodule structure obtained from ${ }_{\alpha_{p}} A^{*}$ through the identification $\widehat{\alpha_{p}(1) a} \mapsto a^{*} \alpha_{p}(1)$.

A representation of a product system $\mathcal{E}=\left(\mathcal{E}_{p}\right)_{p \in P}$ in a $\mathrm{C}^{*}$-algebra $B$ consists of linear maps $\psi_{p}: \mathcal{E}_{p} \rightarrow$ $B$, for all $p \in P \backslash\{e\}$, and a *-homomorphism $\psi_{e}: A \rightarrow B$, satisfying the following two axioms:

(T1) $\psi_{p}(\xi) \psi_{q}(\eta)=\psi_{p q}(\xi \eta)$ for all $p, q \in P, \xi \in \mathcal{E}_{p}$ and $\eta \in \mathcal{E}_{q}$;

(T2) $\psi_{p}(\xi)^{*} \psi_{p}(\eta)=\psi_{e}(\langle\xi \mid \eta\rangle)$ for all $p \in P$ and $\xi, \eta \in \mathcal{E}_{p}$.

If $\psi_{e}$ is faithful, we say that $\psi$ is injective. In this case, the relation (T2) implies that $\left\|\psi_{p}(\xi)\right\|=\|\xi\|$ for all $\xi \in \mathcal{E}_{p}$ and $p \in P$.

Proposition 4.1.4 ([26, Proposition 2.8]). Let $\mathcal{E}$ be a product system over $P$. There is a $\mathrm{C}^{*}$-algebra $\mathcal{T}_{\mathcal{E}}$ with a representation $t: \mathcal{E} \rightarrow \mathcal{T}_{\mathcal{E}}$, so that $\widetilde{t}(\mathcal{E})$ generates $\mathcal{T}_{\mathcal{E}}$ as a $\mathrm{C}^{*}$-algebra and, given a representation $\psi=\left\{\psi_{p}\right\}_{p \in P}$ of $\mathcal{E}$ in a $\mathrm{C}^{*}$-algebra $B$, there is a unique ${ }^{*}$-homomorphism $\widetilde{\psi}: \mathcal{T}_{\mathcal{E}} \rightarrow B$ with $\widetilde{\psi}_{\mathrm{o}} \widetilde{t}_{p}=\psi_{p}$ for all $p \in P$. Moreover, the pair $\left(\mathcal{T}_{\mathcal{E}}, \widetilde{t}\right)$ is unique up to canonical isomorphism.

We call $\mathcal{T}_{\mathcal{E}}$ the Toeplitz algebra of $\mathcal{E}$.

Proof. Let $C$ be the universal *-algebra generated by the set $\left\{(\xi, p) \mid \xi \in \mathcal{E}_{p}, p \in P\right\}$, subject to the relations

$$
(\xi, p) \cdot(\eta, q)=\left(\mu_{p, q}(\xi \otimes \eta), p q\right), \quad(\xi, p)^{*}(\zeta, p)=(\langle\xi \mid \eta\rangle, e)
$$

for all $\xi, \zeta \in \mathcal{E}_{p}, \eta \in \mathcal{E}_{q}$ and for all $p, q \in P$. Let $B$ be a $\mathrm{C}^{*}$-algebra and let $\psi: C \rightarrow B$ be a *-homomorphism. Then

$$
\|\psi((\xi, p))\|^{2}=\left\|\psi((\xi, p))^{*} \psi((\xi, p))\right\|=\|\psi(\langle\xi \mid \xi\rangle)\| \leq\|\langle\xi \mid \xi\rangle\|=\|\xi\|^{2} .
$$

\footnotetext{
${ }^{1}$ If $\alpha_{e}$ is injective, the equality $\alpha_{e}=\alpha_{e} \circ \alpha_{e}$ entails $\alpha_{e}=\mathrm{id}_{A}$.
} 
For $\eta \in C$, we set

$$
\rho_{\infty}(\eta):=\sup _{\rho} \rho(\eta)
$$

where $\rho$ ranges in the collection of all $\mathrm{C}^{*}$-seminorms on $C$. This provides $C$ with a well defined $\mathrm{C}^{*}$-seminorm since $\mathcal{E}$ generates $C$ as a ${ }^{*}$-algebra. We then let $\mathcal{T}_{\mathcal{E}}$ be the completion of $\left(C, \rho_{\infty}\right)$. For each $p \in P$, we obtain a linear map $\widetilde{t}_{p}: \mathcal{E}_{p} \rightarrow \mathcal{T}_{\mathcal{E}}$ by composing the canonical ${ }^{*}$-homomorphism $C \rightarrow \mathcal{T}_{\mathcal{E}}$ with the map $\xi \mapsto(\xi, p)$. Set $\widetilde{t}=\left\{\widetilde{t}_{p}\right\}_{p \in P}$. By construction, this is a representation of $\mathcal{E}$ in $\mathcal{T}_{\mathcal{E}}$ and the pair $\left(\mathcal{T}_{\mathcal{E}}, \widetilde{t}\right)$ satisfies all the required properties.

We will see that $\mathcal{E}$ has a canonical injective representation if $P$ is left-cancellative. In particular, $\rho_{\infty}(\xi)=\|\xi\|$ for all $\xi \in \mathcal{E}_{p}$ and for all $p \in P$.

\subsubsection{Fock representation}

Let $\mathcal{E}=\left(\mathcal{E}_{p}\right)_{p \in P}$ be a product system over a left-cancellative semigroup $P$. Let $\mathcal{E}^{+}$be the right Hilbert $A$-module given by the direct sum of all $\mathcal{E}_{p}$ 's. That is,

$$
\mathcal{E}^{+}=\bigoplus_{p \in P} \mathcal{E}_{p}
$$

Define a representation of $\mathcal{E}$ in $\mathbb{B}\left(\mathcal{E}^{+}\right)$as follows. Given $\xi \in \mathcal{E}_{p}$ and $\eta^{+}=\bigoplus_{s \in P} \eta_{s}$, set

$$
\psi_{p}^{+}(\xi)\left(\eta^{+}\right)_{s}= \begin{cases}\mu_{p, p^{-1} s}\left(\xi \otimes \eta_{s}\right) & \text { if } s \in p P \\ 0 & \text { otherwise }\end{cases}
$$

We view $\mathcal{E}_{p s}$ as the correspondence $\mathcal{E}_{p} \otimes_{A} \mathcal{E}_{s}$ through the correspondence isomorphism $\mu_{p, s}^{-1}$. In this way, $\psi_{p}^{+}(\xi)^{*}(\eta)_{s}$ is the image of $\eta_{p s}$ in $\mathcal{E}_{s}$ under the operator defined on elements of the form $\mu_{p, s}\left(\zeta_{p} \otimes \zeta_{s}\right)$ by the formula

$$
\psi_{p}^{+}(\xi)^{*}\left(\mu_{p, s}\left(\zeta_{p} \otimes \zeta_{s}\right)\right)=\varphi_{s}\left(\left\langle\xi \mid \zeta_{p}\right\rangle\right) \zeta_{s} .
$$

Since $P$ is left-cancellative, this is well defined. So $\psi_{p}^{+}(\xi)^{*}$ is the adjoint of $\psi_{p}^{+}(\xi)$ (see Subsection 3.1.1. This gives rise to a representation $\psi^{+}=\left\{\psi_{p}^{+}\right\}_{p \in P}$ of $\mathcal{E}$ in $\mathbb{B}\left(\mathcal{E}^{+}\right)$called the Fock representation of $\mathcal{E}$.

Corollary 4.1.5. Let $P$ be a left-cancellative semigroup and $\mathcal{E}$ a product system over $P$. Then the universal representation of $\mathcal{E}$ in $\mathcal{T}_{\mathcal{E}}$ is injective.

Proof. It suffices to show that the Fock representation is injective on $A$. This follows from the fact that $\psi_{e}^{+}$is the diagonal ${ }^{*}$-homomorphism from $A$ to $\mathbb{B}\left(\mathcal{E}^{+}\right)$. This is injective because $\mathcal{E}^{+}$contains a copy of $A$ as a direct summand.

\subsubsection{The coaction on the Toeplitz algebra}

The idea of considering coactions on Toeplitz algebras associated to product systems goes back to 26. Proposition 4.7] and also [15. for Nica-Toeplitz algebras. Given a product system $\mathcal{E}=\left(\mathcal{E}_{p}\right)_{p \in P}$ and a discrete group $G$ with $G \supseteq P$, we will need the topological $G$-grading coming from the canonical coaction of $G$ on the Toeplitz algebra $\mathcal{T}_{\mathcal{E}}$ in Chapter 6. Hence we begin with a description of such a grading.

Let $\mathcal{E}=\left(\mathcal{E}_{p}\right)_{p \in P}$ be a product system. Suppose that $P$ is a subsemigroup of a group $G$. There is a representation of $\mathcal{E}$ in $\mathcal{T}_{\mathcal{E}} \otimes C^{*}(G)$ which sends $\xi_{p} \in \mathcal{E}_{p}$ to $\tilde{t}\left(\xi_{p}\right) \otimes u_{p}$. By the universal property of $\mathcal{T}_{\mathcal{E}}$, this yields a ${ }^{*}$-homomorphism $\widetilde{\delta}: \mathcal{T}_{\mathcal{E}} \rightarrow \mathcal{T}_{\mathcal{E}} \otimes C^{*}(G)$. Such a ${ }^{*}$-homomorphism provides $\mathcal{T}_{\mathcal{E}}$ with a topological $G$-grading. This will be a crucial tool for the remainder of this thesis.

Lemma 4.1.6. The ${ }^{*}$-homomorphism $\widetilde{\delta}: \mathcal{T}_{\mathcal{E}} \rightarrow \mathcal{T}_{\mathcal{E}} \otimes C^{*}(G)$ provides a full coaction of $G$ on $\mathcal{T}_{\mathcal{E}}$. Moreover, the spectral subspace $\mathcal{T}_{\mathcal{E}}^{g}$ at $g \in G$ associated to $\widetilde{\delta}$ is the closure of sums of elements of the form

$$
\tilde{t}\left(\xi_{p_{1}}\right) \tilde{t}\left(\xi_{p_{2}}\right)^{*} \ldots \tilde{t}\left(\xi_{p_{n-1}}\right) \tilde{t}\left(\xi_{p_{n}}\right)^{*}
$$

where $n \in \mathbb{N}, p_{1} p_{2}^{-1} \ldots p_{n-1} p_{n}^{-1}=g$ and $\xi_{p_{i}} \in \mathcal{E}_{p_{i}}$ for all $i \in\{1,2, \ldots, n\}$. 
Proof. We begin by proving that $\widetilde{\delta}$ is nondegenerate. Let $\left(u_{\lambda}\right)_{\lambda \in \Lambda}$ be an approximate identity for $A$. For each $p \in P$, both the left and right actions of $A$ on $\mathcal{E}_{p}$ are nondegenerate. Consequently, $\left(\widetilde{t}_{e}\left(u_{\lambda}\right)\right)_{\lambda \in \Lambda}$ is an approximate unit for $\mathcal{T}_{\mathcal{E}}$. Hence its image under $\widetilde{\delta}$ satisfies, for all $b \in \mathcal{T}_{\mathcal{E}}$ and $g \in G$,

$$
\lim _{\lambda} \widetilde{\delta}\left(\widetilde{t}_{e}\left(u_{\lambda}\right)\right)\left(b \otimes u_{g}\right)=\lim _{\lambda}\left(\widetilde{t}_{e}\left(u_{\lambda}\right) \otimes u_{e}\right)\left(b \otimes u_{g}\right)=\lim _{\lambda} \widetilde{t}_{e}\left(u_{\lambda}\right) b \otimes u_{g}=b \otimes u_{g} .
$$

This guarantees that $\widetilde{\delta}$ is nondegenerate. In addition, for all $p \in P$, we have

$$
\left(\widetilde{\delta}_{p} \otimes \operatorname{id}_{C^{*}(G)}\right) \widetilde{\delta}_{p}=\left(\operatorname{id}_{\mathcal{T}_{\mathcal{E}}} \otimes \delta_{G}\right) \widetilde{\delta}_{p}
$$

on $\widetilde{t}\left(\mathcal{E}_{p}\right)$. Thus $\widetilde{\delta}$ satisfies the coaction identity on $\mathcal{T}_{\mathcal{E}}$ as well, because it is generated by $\widetilde{t}(\mathcal{E})$ as a $\mathrm{C}^{*}$-algebra.

It remains to prove that $\widetilde{\delta}$ is injective. Indeed, let $1_{G}: G \rightarrow \mathbb{C}, g \mapsto 1$, be the trivial group homomorphism. Then $\left(\operatorname{id}_{\mathcal{T}_{\mathcal{E}}} \otimes 1_{G}\right) \circ \widetilde{\delta}=\mathrm{id}_{\mathcal{T}_{\mathcal{E}}}$ if we identify $\mathcal{T}_{\mathcal{E}}$ with $\mathcal{T}_{\mathcal{E}} \otimes \mathbb{C}$ in the canonical way. So $\widetilde{\delta}$ is injective. Hence $\widetilde{\delta}$ is a full coaction of $G$ on the Toeplitz algebra of $\mathcal{E}$.

Now let $\mathcal{T}_{\mathcal{E}}^{g}$ be the spectral subspace at $g \in G$ for $\widetilde{\delta}$ and let $\widetilde{\delta}_{g}$ denote the projection of $\mathcal{T}_{\mathcal{E}}$ onto $\mathcal{T}_{\mathcal{E}}^{g}$ as in Proposition 2.2.10. Take $b$ in $\mathcal{T}_{\mathcal{E}}^{g}$. Since $\widetilde{\delta}_{g}$ is contractive and $\mathcal{T}_{\mathcal{E}}$ is generated by $\tilde{t}(\mathcal{E})$ as a $\mathrm{C}^{*}$-algebra, we may suppose that

$$
b=\sum_{j=1}^{m} \tilde{t}\left(\xi_{p_{1}^{j}}\right) \tilde{t}\left(\xi_{p_{2}^{j}}\right)^{*} \ldots \tilde{t}\left(\xi_{p_{k_{j}-1}^{j}}\right) \tilde{t}\left(\xi_{p_{k_{j}}^{j}}\right)^{*}
$$

where $m, k_{j} \in \mathbb{N}$ for all $j$ in $\{1,2, \ldots, m\}$ and $\xi_{p_{i}^{j}} \in \mathcal{E}_{p_{i}^{j}}$. The assertion then follows from the fact that $\widetilde{\delta}_{g}$ vanishes on any element of the form

$$
\tilde{t}\left(\xi_{p_{1}}\right) \tilde{t}\left(\xi_{p_{2}}\right)^{*} \ldots \tilde{t}\left(\xi_{p_{n-1}}\right) \tilde{t}\left(\xi_{p_{n}}\right)^{*}
$$

with $p_{1} p_{2}^{-1} \ldots p_{n-1} p_{n}^{-1} \neq g$.

We call the coaction obtained in the previous lemma as the generalised gauge coaction of $G$ on $\mathcal{T}_{\mathcal{E}}$.

\subsection{Compactly aligned product systems}

Nica-Toeplitz algebras are defined for a class of product systems over positive cones of quasi-lattice ordered groups. They are quotients of Toeplitz algebras and carry a grading as in Lemma 4.1.6. In this section, we look at the canonical topological grading of a Nica-Toeplitz algebra and, more generally, of a relative Cuntz-Pimsner algebra. This grading has special features, which led us to study the class of Fell bundles introduced in Chapter 5

\subsubsection{Nica-Toeplitz algebras}

Let us restrict our attention to semigroups arising from quasi-lattice orders in the sense of [47: let $G$ be a group and let $P$ be a subsemigroup of $G$ with $P \cap P^{-1}=\{e\}$. We say that $(G, P)$ is a quasi-lattice ordered group if any two elements $g_{1}, g_{2}$ of $G$ with a common upper bound in $P$ with respect to the partial order $g_{1} \leq g_{2} \Leftrightarrow g_{1}^{-1} g_{2} \in P$ also have a least upper bound $g_{1} \vee g_{2}$ in $P$. We write $g_{1} \vee g_{2}=\infty$ if $g_{1}$ and $g_{2}$ have no common upper bound in $P$. Following [17], we call $P$ the positive cone of $(G, P)$, observing that $P=\{g \in G \mid g \geq e\}$.

If $(G, P)$ is a quasi-lattice ordered group, any element $g$ in $G$ satisfying $g \vee e<\infty$ has a certain reduced form $p q^{-1}$ with $p, q \in P$, which will allow us in the subsequent chapter to extend a class of product systems of Hilbert bimodules over $P$ to Fell bundles over $G$ in a natural way.

Lemma 4.2.1. Let $(G, P)$ be a quasi-lattice ordered group and let $g \in G$ with $g \vee e<\infty$. Then $g^{-1} \vee e<\infty$ and $g=(g \vee e)\left(g^{-1} \vee e\right)^{-1}$.

Proof. Let $q \in P$ be such that $g^{-1}(g \vee e)=q$. Then $g \vee e=g q=\left(g^{-1}\right)^{-1} q$. This shows that $g^{-1} \vee e<\infty$ and $g^{-1} \vee e \leq q$. But $g\left(g^{-1} \vee e\right)$ belongs to $P$ and $g \leq g\left(g^{-1} \vee e\right)$. So $g \vee e \leq g\left(g^{-1} \vee e\right)$. 
Since the partial order $g_{1} \leq g_{2} \Leftrightarrow g_{1}^{-1} g_{2} \in P$ is invariant under left-translation by elements of $G$, it follows that $q=g^{-1}(g \vee e) \leq g^{-1} \vee e$. Therefore, $g=(g \vee e)\left(g^{-1} \vee e\right)^{-1}$.

Example 4.2.2. Let $k \in \mathbb{N}^{+}$. Then $\left(\mathbb{Z}^{k}, \mathbb{N}^{k}\right)$ is quasi-lattice ordered. For this example, $m \leq n$ if and only if $m_{i} \leq n_{i}$ for all $i \in\{1,2, \ldots, k\}$, where $m, n \in \mathbb{Z}^{k}$.

Example 4.2.3. Let $\mathbb{F}$ be the free group on the set of generators $S$. Let $\mathbb{F}^{+}$be the unital subsemigroup of $\mathbb{F}$ generated by $S$. Any element of $\mathbb{F}$ has a reduced form $a_{1} a_{2} \cdots a_{n}$ with $a_{i} \in S \cup S^{-1}$ for all $i \in\{1,2, \ldots, n\}$ and $a_{i} \neq a_{i+1}^{-1}$ for all $i \in\{1,2, \ldots, n-1\}$. Hence one can prove that $\left(\mathbb{F}, \mathbb{F}^{+}\right)$is a quasi-lattice ordered group (see [47]). For $g \in \mathbb{F}, g \vee e<\infty$ if and only if its reduced form is $p q^{-1}$ with $p$ and $q$ in $\mathbb{F}^{+}$. In this case, $p=g \vee e$ and $q=g^{-1} \vee e$.

Example 4.2.4. Let $c$ and $d$ be nonzero integers. The Baumslag-Solitar group BS $(c, d)$ is the universal group on two generators $a$ and $b$ subject to the relation $a b^{c}=b^{d} a$. If $c, d>0$, the unital subsemigroup of $\operatorname{BS}(c, d)$ generated by $a$ and $b$ provides it with a partial order so that $\left(\operatorname{BS}(c, d), \operatorname{BS}(c, d)^{+}\right)$is a quasi-lattice ordered group (see [56, Theorem 2.11]).

Let $(G, P)$ be a quasi-lattice ordered groups and let $\mathcal{E}=\left(\mathcal{E}_{p}\right)_{p \in P}$ be a product system over $P$. Let $\psi=\left\{\psi_{p}\right\}_{p \in P}$ be a representation of $\mathcal{E}$ in a $\mathrm{C}^{*}$-algebra $B$. For each $p \in P$, we will denote by $\psi^{(p)}$ the ${ }^{*}$-homomorphism from $\mathbb{K}\left(\mathcal{E}_{p}\right)$ to $B$ obtained as in [48]. This is defined on a generator $|\xi\rangle\langle\eta|$ by

$$
\psi^{(p)}(|\xi\rangle\langle\eta|):=\psi_{p}(\xi) \psi_{p}(\eta)^{*} .
$$

We may use the multiplication maps on $\mathcal{E}$ to define ${ }^{*}$-homomorphisms $\iota_{p}^{p q}: \mathbb{B}\left(\mathcal{E}_{p}\right) \rightarrow \mathbb{B}\left(\mathcal{E}_{p q}\right)$. Explicitly, $\iota_{p}^{p q}$ sends $T \in \mathbb{B}\left(\mathcal{E}_{p}\right)$ to $\mu_{p, q} \circ\left(T \otimes \mathrm{id}_{\mathcal{E}_{q}}\right) \circ \mu_{p, q}^{-1}$. We say that $\mathcal{E}=\left(\mathcal{E}_{p}\right)_{p \in P}$ is compactly aligned if, for all $p, q \in P$ with $p \vee q<\infty$, we have

$$
\iota_{p}^{p \vee q}(T) \iota_{q}^{p \vee q}(S) \in \mathbb{K}\left(\mathcal{E}_{p \vee q}\right), \quad \text { for all } T \in \mathbb{K}\left(\mathcal{E}_{p}\right) \text { and } S \in \mathbb{K}\left(\mathcal{E}_{q}\right) .
$$

If $\mathcal{E}$ is compactly aligned, a representation $\psi=\left\{\psi_{p}\right\}_{p \in P}$ of $\mathcal{E}$ in a $\mathrm{C}^{*}$-algebra $B$ is Nica covariant if, for all $p, q \in P, T \in \mathbb{K}\left(\mathcal{E}_{p}\right)$ and $S \in \mathbb{K}\left(\mathcal{E}_{q}\right)$, we have

$$
\psi^{(p)}(T) \psi^{(q)}(S)= \begin{cases}\psi^{(p \vee q)}\left(\iota_{p}^{p \vee q}(T) \iota_{q}^{p \vee q}(S)\right) & \text { if } p \vee q<\infty \\ 0 & \text { otherwise. }\end{cases}
$$

Proposition 4.2.5 ([26, Theorem 6.3]). Let $(G, P)$ be a quasi-lattice ordered group and let $\mathcal{E}$ be a compactly aligned product system over $P$. Then there is a $\mathrm{C}^{*}$-algebra $\mathcal{N} \mathcal{T}_{\mathcal{E}}$ and a Nica covariant representation $\bar{\pi}=\left\{\bar{\pi}_{p}\right\}_{p \in P}$ of $\mathcal{E}$ in $\mathcal{N} \mathcal{T}_{\mathcal{E}}$ so that $\bar{\pi}(\mathcal{E})$ generates $\mathcal{N} \mathcal{T}_{\mathcal{E}}$ as a $\mathrm{C}^{*}$-algebra and, given a Nica covariant representation $\psi=\left\{\psi_{p}\right\}_{p \in P}$ of $\mathcal{E}$ in a $\mathrm{C}^{*}$-algebra $B$, there is a unique ${ }^{*}$-homomorphism $\bar{\psi}: \mathcal{T}_{\mathcal{E}} \rightarrow B$ such that $\bar{\psi} \circ \bar{\pi}_{p}=\psi_{p}$ for all $p \in P$. Moreover, $\bar{\pi}$ is injective and the pair $\left(\mathcal{N} \mathcal{T}_{\mathcal{E}}, \bar{\pi}\right)$ is unique up to canonical isomorphism.

Proof. Let $I_{\mathcal{N}}$ be the ideal in $\mathcal{T}_{\mathcal{E}}$ generated by the union of

$$
\left\{\widetilde{t}^{(p)}(T) \widetilde{t}^{(q)}(S)-\widetilde{t}^{(p \vee q)}\left(\iota_{p}^{p \vee q}(T) \iota_{q}^{p \vee q}(S)\right) \mid p, q \in P, p \vee q<\infty, T \in \mathbb{K}\left(\mathcal{E}_{p}\right) \text { and } S \in \mathbb{K}\left(\mathcal{E}_{q}\right)\right\}
$$

with

$$
\left\{\widetilde{t}^{(p)}(T) \widetilde{t}^{(q)}(S) \mid p, q \in P, p \vee q=\infty, T \in \mathbb{K}\left(\mathcal{E}_{p}\right) \text { and } S \in \mathbb{K}\left(\mathcal{E}_{q}\right)\right\} .
$$

Put $\mathcal{N} \mathcal{T}_{\mathcal{E}}:=\mathcal{T}_{\mathcal{E}} / I_{\mathcal{N}}$ and let $\bar{\pi}=\left\{\bar{\pi}_{p}\right\}_{p \in P}$ be the representation of $\mathcal{E}$ in $\mathcal{N} \mathcal{T}_{\mathcal{E}}$ obtained from the composition of $\widetilde{t}$ with the quotient map. So $\bar{\pi}$ is Nica covariant. It is also injective because the Fock representation is Nica covariant. Hence the pair $\left(\mathcal{N} \mathcal{T}_{\mathcal{E}}, \bar{\pi}\right)$ satisfies all the required properties.

We call $\mathcal{N} \mathcal{T}_{\mathcal{E}}$ the $N i c a-T o e p l i t z$ algebra of $\mathcal{E}$.

\subsubsection{Relative Cuntz-Pimsner algebras}

Let $\mathcal{E}=\left(\mathcal{E}_{p}\right)_{p \in P}$ be a product system. For each $p \in P$, let $J_{p} \triangleleft A$ be an ideal that acts by compact operators on $\mathcal{E}_{p}$ and set $\mathcal{J}=\left\{J_{p}\right\}_{p \in P}$. We say that a representation $\psi=\left\{\psi_{p}\right\}_{p \in P}$ is Cuntz-Pimsner covariant on $\mathcal{J}$ if, for all $p \in P$ and all $a$ in $J_{p}$,

$$
\psi^{(p)}\left(\varphi_{p}(a)\right)=\psi_{e}(a)
$$


Repeating the argument employed in the proof of Proposition 4.2.5, we obtain the following:

Proposition 4.2.6. Let $(G, P)$ be a quasi-lattice ordered group and let $\mathcal{E}$ be a compactly aligned product system over $P$. Let $\mathcal{J}=\left\{J_{p}\right\}_{p \in P}$ be a family of ideals in $A$ with $\varphi_{p}\left(J_{p}\right) \subseteq \mathbb{K}\left(\mathcal{E}_{p}\right)$ for all $p \in P$. Then there is a $\mathrm{C}^{*}$-algebra $\mathcal{O}_{\mathcal{J}, \mathcal{E}}$ and a Nica covariant representation $j=\left\{j_{p}\right\}_{p \in P}$ of $\mathcal{E}$ in $\mathcal{O}_{\mathcal{J}, \mathcal{E}}$ that is also Cuntz-Pimsner covariant on $\mathcal{J}$ and such that

(i) $\mathcal{O}_{\mathcal{J}, \mathcal{E}}$ is generated by $j(\mathcal{E})$ as a $\mathrm{C}^{*}$-algebra;

(ii) given a Nica covariant representation $\psi=\left\{\psi_{p}\right\}_{p \in P}$ of $\mathcal{E}$ in a $\mathrm{C}^{*}$-algebra $B$ that is Cuntz-Pimsner covariant on $\mathcal{J}$, there is a unique ${ }^{*}$-homomorphism $\bar{\psi}_{\mathcal{J}}: \mathcal{O}_{\mathcal{J}, \mathcal{E}} \rightarrow B$ such that $\bar{\psi}_{\mathcal{J}} \circ j_{p}=\psi_{p}$ for all $p \in P$.

Moreover, the pair $\left(\mathcal{O}_{\mathcal{J}, \mathcal{E}}, j\right)$ is unique up to canonical isomorphism.

Definition 4.2.7. Given $\mathcal{E}$ and $\mathcal{J}$ as above, we call $\mathcal{O}_{\mathcal{J}, \mathcal{E}}$ the relative Cuntz-Pimsner algebra determined by $\mathcal{J}$.

We emphasize two particular cases. If $J_{p}=\{0\}$ for all $p \in P$, then $\mathcal{O}_{\mathcal{J}, \mathcal{E}}=\mathcal{N} \mathcal{T}_{\mathcal{E}}$. If $(G, P)=(\mathbb{Z}, \mathbb{N})$, $\mathcal{E}$ is a product system of Hilbert bimodules and $J_{p}=\left\langle\left\langle\mathcal{E}_{p} \mid \mathcal{E}_{p}\right\rangle\right\rangle$ for all $p$ in $P$, then $\mathcal{O}_{\mathcal{J}, \mathcal{E}}$ is the $\mathrm{C}^{*}$-algebra studied by Katsura in [29]. He proved that the canonical *-homomorphism from $A$ to $\mathcal{O}_{\mathcal{J}, \mathcal{E}}$ is an isomorphism onto the fixed-point algebra of $\mathcal{O}_{\mathcal{J}, \mathcal{E}}$. In this case, $\mathcal{E}$ extends to a semi-saturated Fell bundle over $\mathbb{Z}$ (see [1]). We will generalise this to a certain class of compactly aligned product systems of Hilbert bimodules over semigroups arising from quasi-lattice orders.

Remark 4.2.8. Fowler defined the Cuntz-Pimsner algebra of a product system $\mathcal{E}$ to be the universal $\mathrm{C}^{*}$-algebra for representations of $\mathcal{E}$ that are Cuntz-Pimsner covariant on $\mathcal{J}=\left\{J_{p}\right\}_{p \in P}$, where $J_{p}=\varphi_{p}^{-1}\left(\mathbb{K}\left(\mathcal{E}_{p}\right)\right)$ for all $p \in P$ (see [26]). Here we consider the class of compactly aligned product systems and define the relative Cuntz-Pimsner algebra with respect to a family of ideals as a quotient of the Nica-Toeplitz algebra of $\mathcal{E}$. This provides the construction of relative Cuntz-Pimsner algebras with a special feature and will allow us to generalise most of the results obtained in Chapter 3 to quasi-lattice ordered groups. Our approach applies to Fowler's Cuntz-Pimsner algebras of proper product systems $\mathcal{E}=\left(\mathcal{E}_{p}\right)_{p \in P}$ if $(G, P)$ is a quasi-lattice ordered group and $P$ is directed. This is so because, in this case, a Cuntz-Pimsner covariant representation of $\mathcal{E}$ in the sense of Fowler is also Nica covariant 26, Proposition 5.4].

A product system of Hilbert bimodules $\mathcal{E}=\left(\mathcal{E}_{p}\right)_{p \in P}$ gives rise to a product system $\mathcal{E}^{*}$ over $P^{\text {op }}$ by setting $\mathcal{E}^{*}:=\left(\mathcal{E}_{p}^{*}\right)_{p \in P}$, where $\mathcal{E}_{p}^{*}$ is the Hilbert bimodule adjoint to $\mathcal{E}_{p}$. We will identify $A$ with its

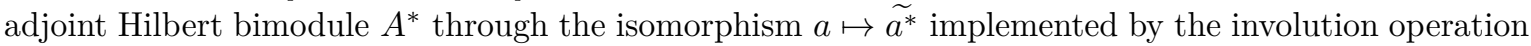
on $A$, where $\widetilde{a^{*}}$ is the image of $a^{*}$ in $A^{*}$ under the canonical conjugate-linear map. The multiplication map $\mathcal{E}_{p}^{*} \otimes_{A} \mathcal{E}_{q}^{*} \cong \mathcal{E}_{q p}^{*}$ is given by the isomorphism $\mathcal{E}_{p}^{*} \otimes_{A} \mathcal{E}_{q}^{*} \cong\left(\mathcal{E}_{q} \otimes_{A} \mathcal{E}_{p}\right)^{*}, \xi^{*} \otimes \eta^{*} \mapsto(\eta \otimes \xi)^{*}$, followed by the multiplication map $\mu_{q, p}$. In addition, $\mathcal{E}^{* *}=\mathcal{E}$. Before providing more concrete examples of relative Cuntz-Pimsner algebras, we need the following lemma.

Lemma 4.2.9. Let $\mathcal{E}=\left(\mathcal{E}_{p}\right)_{p \in P}$ be a product system of Hilbert bimodules. For each $p \in P$, let $I_{\mathcal{E}_{p}}:=$ $\left\langle\left\langle\mathcal{E}_{p} \mid \mathcal{E}_{p}\right\rangle\right\rangle$ and set $\mathcal{I}_{\mathcal{E}}=\left\{I_{\mathcal{E}_{p}}\right\}_{p \in P}$. A representation $\psi=\left\{\psi_{p}\right\}_{p \in P}$ of $\mathcal{E}$ in a $\mathrm{C}^{*}$-algebra $B$ that is Cuntz-Pimsner covariant on $\mathcal{I}_{\mathcal{E}}$ naturally induces a representation of $\mathcal{E}^{*}=\left(\mathcal{E}_{p}^{*}\right)_{p \in P}$ that is CuntzPimsner covariant on $\mathcal{I}_{\mathcal{E}^{*}}$, where $\mathcal{I}_{\mathcal{E}^{*}}=\left\{I_{\mathcal{E}_{p}^{*}}\right\}_{p \in P}$ and $I_{\mathcal{E}_{p}^{*}}=\left\langle\left\langle\mathcal{E}_{p}^{*} \mid \mathcal{E}_{p}^{*}\right\rangle\right\rangle=\left\langle\mathcal{E}_{p} \mid \mathcal{E}_{p}\right\rangle$. As a consequence, representations of $\mathcal{E}$ that are Cuntz-Pimsner covariant on $\mathcal{I}_{\mathcal{E}}$ are in one-to-one correspondence with representations of $\mathcal{E}^{*}$ that are Cuntz-Pimsner covariant on $\mathcal{I}_{\mathcal{E}^{*}}$.

Proof. For $p=e$, put $\psi_{e}^{*}:=\psi_{e}$. Given $p \in P \backslash\{e\}$, define $\psi_{p}^{*}: \mathcal{E}_{p}^{*} \rightarrow B$ by $\psi_{p}^{*}\left(\xi^{*}\right):=\psi_{p}(\xi)^{*}$ and set $\psi^{*}=\left\{\psi_{p}^{*}\right\}_{p \in P}$. Then, for all $\xi \in \mathcal{E}_{p}$ and $\eta \in \mathcal{E}_{q}$,

$$
\psi_{p}^{*}\left(\xi^{*}\right) \psi_{q}^{*}\left(\eta^{*}\right)=\psi_{p}(\xi)^{*} \psi_{q}(\eta)^{*}=\psi_{q p}\left(\mu_{q, p}(\eta \otimes \xi)\right)^{*}=\psi_{q p}^{*}\left(\mu_{q, p}(\eta \otimes \xi)^{*}\right) .
$$

Since $\psi$ is Cuntz-Pimsner covariant on $\mathcal{I}_{\mathcal{E}}$, it follows that

$$
\psi_{p}^{*}\left(\xi^{*}\right)^{*} \psi_{p}^{*}\left(\eta^{*}\right)=\psi_{p}(\xi) \psi_{p}(\eta)^{*}=\psi^{(p)}(|\xi\rangle\langle\eta|)=\psi_{e}(\langle\langle\xi \mid \eta\rangle\rangle)=\psi_{e}^{*}(\langle\langle\xi \mid \eta\rangle\rangle)
$$

for all $\xi, \eta \in \mathcal{E}_{p}$. That $\psi^{*}$ is Cuntz-Pimsner covariant on $\mathcal{I}_{\mathcal{E}^{*}}$ follows from the fact that $\psi$ is a representation of $\mathcal{E}$. So the last statement is obtained from the identity $\mathcal{E}=\mathcal{E}^{* *}$. 


\section{PRODUCT SYSTEMS AND SOME C*-CONSTRUCTIONS}

Example 4.2.10. Let $\alpha: P \rightarrow \operatorname{End}(A)$ be an action by injective extendible endomorphisms with hereditary range. Let $A_{\alpha}=\left(A_{\alpha_{p}}\right)_{p \in P}$ be the product system of Hilbert bimodules built out of $\alpha$ as in Example 4.1.3 Although it is not clear when $A_{\alpha}$ is compactly aligned, ${ }_{\alpha} A$ always is so. The ideal $I_{p} \triangleleft A$ given by the left inner product of $A_{\alpha_{p}}$ is precisely $\overline{A \alpha_{p}(1) A}$. Given a nondegenerate representation $\psi=$ $\left\{\psi_{p}\right\}_{p \in P}$ of $A_{\alpha}$ in a $\mathrm{C}^{*}$-algebra $B$, we obtain a strictly continuous unital *-homomorphism $\bar{\psi}_{e}: M(A) \rightarrow$ $M(B)$ by nondegeneracy of $\psi_{e}$. In addition, we define a semigroup homomorphism from $P$ to the semigroup of isometries in $M(B)$ by setting

$$
v_{p}:=\lim _{\lambda} \psi_{p}\left(u_{\lambda} \alpha_{p}(1)\right)
$$

Here the limit is taken in the strict topology of $M(B)$. It indeed exists because $\left\|\psi_{p}\left(u_{\lambda} \alpha_{p}(1)\right)\right\| \leq 1$ for each $\lambda$ and, for $a \in A$ and $b \in B$,

$$
\lim _{\lambda} \psi_{p}\left(u_{\lambda} \alpha_{p}(1)\right)\left(\psi_{e}(a) b\right)=\lim _{\lambda} \psi_{p}\left(u_{\lambda} \alpha_{p}(a)\right) b=\psi_{p}\left(\alpha_{p}(a)\right) b
$$

and

$$
\lim _{\lambda}\left(b \psi_{e}(a)\right) \psi_{p}\left(u_{\lambda} \alpha_{p}(1)\right)=\lim _{\lambda} b \psi_{p}\left(a u_{\lambda} \alpha_{p}(1)\right)=b \psi_{p}\left(a \alpha_{p}(1)\right) .
$$

To see that $v_{p}^{*} v_{p}=1$, observe that

$$
v_{p}^{*} v_{p}\left(\psi_{e}(a) b\right)=\lim _{\lambda} \psi_{p}\left(u_{\lambda} \alpha_{p}(1)\right)^{*} \psi_{p}\left(\alpha_{p}(a)\right) b=\lim _{\lambda} \psi_{e}\left(\alpha_{p}^{-1}\left(\alpha_{p}(1) u_{\lambda} \alpha_{p}(a)\right)\right) b=\psi_{e}(a) b .
$$

The semigroup of isometries $\left\{v_{p} \mid p \in P\right\}$ and the ${ }^{*}$-homomorphism $\bar{\psi}_{e}: M(A) \rightarrow M(B)$ satisfy the relation

$$
v_{p} \cdot \bar{\psi}_{e}(c)=\bar{\psi}_{e}\left(\alpha_{p}(c)\right) v_{p}
$$

for all $c \in M(A)$ and $p \in P$. Hence

$$
\bar{\psi}_{e}\left(\alpha_{p}(c)\right) v_{p} v_{p}^{*}=v_{p} \bar{\psi}_{e}(c) v_{p}^{*}
$$

In addition, $\psi_{p}\left(a \alpha_{p}(1)\right)=\psi_{e}(a) v_{p}$ for all $a \in A$ and $p \in P$. If $\psi$ is Cuntz-Pimsner covariant on $\mathcal{I}_{A_{\alpha}}=\left\{I_{p}\right\}_{p \in P}$, it follows that for all $c \in M(A)$ and $p \in P$,

$$
\bar{\psi}_{e}\left(\alpha_{p}(c)\right)=\bar{\psi}_{e}\left(\alpha_{p}(c)\right) v_{p} v_{p}^{*}
$$

Indeed, for $c$ in $A$ and $a \alpha_{p}(1)$ in $A_{\alpha_{p}}$, we compute

$$
\begin{aligned}
\alpha_{p}\left(c^{*} c\right) a \alpha_{p}(1) & =\alpha_{p}\left(c^{*}\right) \alpha_{p}\left(\alpha_{p^{-1}}\left(\alpha_{p}(c) a \alpha_{p}(1)\right)\right) \\
& =\alpha_{p}\left(c^{*}\right) \alpha_{p}\left(\left\langle\alpha_{p}\left(c^{*}\right) \alpha_{p}(1) \mid a \alpha_{p}(1)\right\rangle\right) \\
& =\alpha_{p}\left(c^{*}\right) \cdot\left\langle\alpha_{p}\left(c^{*}\right) \alpha_{p}(1) \mid a \alpha_{p}(1)\right\rangle \\
& =\left|\alpha_{p}\left(c^{*}\right)\right\rangle\left\langle\alpha_{p}\left(c^{*}\right)\right|\left(a \alpha_{p}(1)\right) .
\end{aligned}
$$

Hence Cuntz-Pimsner covariance gives us

$$
\begin{aligned}
\psi_{e}\left(\alpha_{p}\left(c^{*} c\right)\right) & =\psi_{p}\left(\alpha_{p}\left(c^{*}\right)\right) \psi_{p}\left(\alpha_{p}\left(c^{*}\right)\right)^{*}=\psi_{e}\left(\alpha_{p}\left(c^{*}\right)\right) v_{p} v_{p}^{*} \psi_{e}\left(\alpha_{p}(c)\right) \\
& =\psi_{e}\left(\alpha_{p}\left(c^{*}\right)\right) v_{p} \psi_{e}(c) v_{p}^{*}=\psi_{e}\left(\alpha_{p}\left(c^{*} c\right)\right) v_{p} v_{p}^{*}
\end{aligned}
$$

Since $A$ is spanned by positive elements, the same relation holds for all $c \in A$ and thus for all $c \in M(A)$ if we replace $\psi_{e}$ by its extension $\bar{\psi}_{e}$. So combining this with 4.2 .11 , we deduce the relation

$$
\psi_{e}\left(\alpha_{p}(c)\right)=v_{p} \psi_{e}(c) v_{p}^{*}
$$

for all $c \in A$. The same holds for $\bar{\psi}_{e}$ and $c$ in $M(A)$.

Conversely, we claim that a nondegenerate ${ }^{*}$-homomorphism $\pi: A \rightarrow B$ together with a semigroup of isometries $\left\{v_{p} \mid p \in P\right\}$ satisfying the relation

$$
\pi\left(\alpha_{p}(a)\right)=v_{p} \pi(a) v_{p}^{*}
$$


yields a representation of $A_{\alpha}$ that is Cuntz-Pimsner covariant on $\mathcal{I}_{A_{\alpha}}$. First, notice that the projection $v_{p} v_{p}^{*}$ coincides with $\bar{\pi}\left(\alpha_{p}(1)\right)$, where $\bar{\pi}$ is the strictly continuous ${ }^{*}$-homomorphism $M(A) \rightarrow M(B)$ extending $\pi$. For each $p \in P$ and $a \in A$, we set $\psi_{p}\left(a \alpha_{p}(1)\right):=\pi(a) v_{p}$. Put $\psi=\left\{\psi_{p}\right\}_{p \in P}$. Then $\bar{\pi}\left(\alpha_{p}(1)\right)=v_{p} v_{p}^{*}$ implies that $\psi$ is Cuntz-Pimsner covariant on $I_{p}=\overline{A \alpha_{p}(1) A}$ for all $p \in P$, since

$$
\begin{aligned}
\psi_{e}\left(a \alpha_{p}(1) b\right) & =\pi\left(a \alpha_{p}(1) b\right)=\pi(a) \bar{\pi}(1) \pi(b)=\pi(a) v_{p} v_{p}^{*} \pi(b)=\pi(a) v_{p}\left(\pi\left(b^{*}\right) v_{p}\right)^{*} \\
& =\psi_{p}\left(a \alpha_{p}(1)\right) \psi_{p}\left(b^{*} \alpha_{p}(1)\right)^{*}=\psi^{(p)}\left(\left|a \alpha_{p}(1)\right\rangle\left\langle b^{*} \alpha_{p}(1)\right|\right)
\end{aligned}
$$

for all $a$ and $b$ in $A$. Moreover, 4.2.12 tells us that $\psi_{e}\left(\alpha_{p}(a)\right) v_{p}=v_{p} \psi_{e}(a)$ for all $a \in A$ and $p \in P$. This also gives

$$
\psi_{p}\left(a \alpha_{p}(1)\right) \psi_{q}\left(b \alpha_{q}(1)\right)=\psi_{p q}\left(a \alpha_{p}(b) \alpha_{p q}(1)\right)=\psi_{p q}\left(\mu_{p, q}\left(a \alpha_{p}(1) \otimes b \alpha_{q}(1)\right)\right) .
$$

Again by 4.2.12,

$$
\begin{aligned}
\psi_{e}\left(\alpha_{p^{-1}}\left(\alpha_{p}(1) a^{*} b \alpha_{p}(1)\right)\right) & =v_{p}^{*} v_{p} \psi_{e}\left(\alpha_{p^{-1}}\left(\alpha_{p}(1) a^{*} b \alpha_{p}(1)\right)\right) v_{p}^{*} v_{p} \\
& =v_{p}^{*} \psi_{e}\left(\alpha_{p}(1) a^{*} b \alpha_{p}(1)\right) v_{p} \\
& =v_{p}^{*} \psi_{e}\left(a^{*} b\right) v_{p} .
\end{aligned}
$$

This shows that $\psi$ is a representation of $A_{\alpha}$ that is Cuntz-Pimsner covariant on $\mathcal{I}_{A_{\alpha}}$.

As a result, the crossed product $A \rtimes_{\alpha} P$ of $A$ by the semigroup of endomorphisms provided by $\alpha$ has a description as the universal $\mathrm{C}^{*}$-algebra of representations of $A_{\alpha}$ that are Cuntz-Pimsner covariant on $\mathcal{I}_{A_{\alpha}}$. By Lemma 4.2.9 $A \rtimes_{\alpha} P$ may also be described as the universal $\mathrm{C}^{*}$-algebra for representations of ${ }_{\alpha} A$ that are Cuntz-Pimsner covariant on $\mathcal{I}_{\alpha} A$. If $P^{\text {op }}$ is the positive cone of a quasi-lattice order and is also directed, a representation of ${ }_{\alpha} A$ that is Cuntz-Pimsner covariant on $\mathcal{I}_{\alpha} A$ is also Nica covariant by 26, Proposition 5.4]. In this case $\mathcal{O}_{\mathcal{I}_{\alpha} A,{ }_{\alpha} A} \cong A \rtimes_{\alpha} P$. In general, $A \rtimes_{\alpha} \stackrel{\alpha}{P}$ is the Cuntz-Pimsner algebra of ${ }_{\alpha} A$ as defined by Fowler [26]. See, for instance, [35] and [37] for constructions of crossed products by semigroups of endomorphisms. We also refer the reader to [38] for this and further constructions of crossed products out of product systems.

Example 4.2.13. We may attach a $\mathrm{C}^{*}$-algebra to a quasi-lattice ordered group $(G, P)$ by considering the trivial product system over $P$. The Toeplitz algebra of $(G, P)$ as introduced by Nica [47], denoted by $\mathrm{C}^{*}(G, P)$, is the Nica-Toeplitz algebra of the trivial product system over $P$. This is the relative Cuntz-Pimsner algebra with respect to the trivial family of ideals $J_{p}=\{0\}$ for all $p \in P$. In fact, there is also a description of $\mathrm{C}^{*}(G, P)$ as a semigroup crossed product as in the previous example (see [35] and also Subsection 6.3.3. This is the universal $\mathrm{C}^{*}$-algebra generated by a family of isometries $\left\{v_{p}\right\}_{p \in P}$ subject to the relation

$$
v_{p} v_{p}^{*} v_{q} v_{q}^{*}= \begin{cases}v_{p \vee q} v_{p \vee q}^{*} & \text { if } p \vee q<\infty, \\ 0 & \text { otherwise. }\end{cases}
$$

The Fock representation in this case is the canonical representation of $P$ by isometries on $\ell_{2}(P)$. The image of $\mathrm{C}^{*}(G, P)$ in $\mathbb{B}\left(\ell_{2}(P)\right)$ under the Fock representation is called Wiener-Hopf algebra [47].

\subsubsection{Coaction on relative Cuntz-Pimsner algebras}

Let $(G, P)$ be a quasi-lattice order and let $\mathcal{E}$ be a compactly aligned product system over $P$. The representation of $\mathcal{E}$ in $\mathcal{O}_{\mathcal{J}, \mathcal{E}} \otimes C^{*}(G)$ which sends $\xi \in \mathcal{E}_{p}$ to $\xi \otimes u_{p}$ is Nica covariant and also CuntzPimsner covariant on $\mathcal{J}$. So this yields a ${ }^{*}$-homomorphism $\delta: \mathcal{O}_{\mathcal{J}, \mathcal{E}} \rightarrow \mathcal{O}_{\mathcal{J}, \mathcal{E}} \otimes C^{*}(G)$.

Proposition 4.2.14. The ${ }^{*}$-homomorphism $\delta: \mathcal{O}_{\mathcal{J}, \mathcal{E}} \rightarrow \mathcal{O}_{\mathcal{J}, \mathcal{E}} \otimes C^{*}(G)$ gives a full coaction of $G$ on $\mathcal{O}_{\mathcal{J}, \mathcal{E}}$. Moreover, the spectral subspace $\mathcal{O}_{\mathcal{J}, \mathcal{E}}^{g}$ for $\delta$ at $g \in G$ with $g \vee e<\infty$ is the closure of sums of elements of the form

$$
j_{p}(\xi) j_{q}(\eta)^{*}
$$

with $\xi \in \mathcal{E}_{p}$ and $\eta \in \mathcal{E}_{q}$, where $p q^{-1}=g$ and $p, q \in P$. If $g \vee e=\infty$, then $\mathcal{O}_{\mathcal{J}, \mathcal{E}}^{g}$ is the trivial subspace.

Proof. The first part of the proof is essentially identical to that of Lemma 4.1.6. For the last part, notice that the Nica covariance condition entails $j_{p}\left(\mathcal{E}_{p}\right)^{*} j_{q}\left(\mathcal{E}_{q}\right)=\{0\}$ whenever $p \vee q=\infty$ because 
$j_{p}\left(\mathcal{E}_{p}\right)=j_{p}\left(\mathcal{E}_{p}\right) j_{p}\left(\mathcal{E}_{p}\right)^{*} j_{p}\left(\mathcal{E}_{p}\right)$ for all $p \in P$. In case $p \vee q<\infty$, we have

$$
j_{p}\left(\mathcal{E}_{p}\right)^{*} j_{q}\left(\mathcal{E}_{q}\right) \subseteq \overline{\operatorname{span}}\left\{j_{p^{-1}(p \vee q)}(\xi) j_{q^{-1}(p \vee q)}(\eta)^{*} \mid \xi \in \mathcal{E}_{p^{-1}(p \vee q)}, \eta \in \mathcal{E}_{q^{-1}(p \vee q)}\right\}
$$

So take $g \in G$ with $g \vee e=\infty$. In particular, $g$ has no presentation of the form $p q^{-1}$ with $p, q$ in $P$. Thus, by successive applications of the above simplification for elements of the form $j_{p}\left(\xi_{p}\right)^{*} j_{q}\left(\xi_{q}\right)$, it follows that

$$
j_{p_{1}}\left(\xi_{p_{1}}\right) j_{p_{2}}\left(\xi_{p_{2}}\right)^{*} \ldots j_{p_{2 n-1}}\left(\xi_{p_{2 n-1}}\right) j_{p_{2 n}}\left(\xi_{p_{2 n}}\right)^{*}=0
$$

whenever $p_{1} p_{2}^{-1} \ldots p_{2 n-1} p_{2 n}^{-1}=g$ and $\xi_{p_{i}} \in \mathcal{E}_{p_{i}}$ for all $i \in\{1,2, \ldots, 2 n\}$. As a consequence, $\mathcal{O}_{\mathcal{J}, \mathcal{E}}^{g}=\{0\}$. Now a similar reasoning shows that if $g \in G$ satisfies $g \vee e<\infty$ and $p_{1} p_{2}^{-1} \ldots p_{2 n-1} p_{2 n}^{-1}=g$, then $j_{p_{1}}\left(\xi_{p_{1}}\right) j_{p_{2}}\left(\xi_{p_{2}}\right)^{*} \ldots j_{p_{2 n-1}}\left(\xi_{p_{2 n-1}}\right) j_{p_{2 n}}\left(\xi_{p_{2 n}}\right)^{*}$ lies in the closed linear span of

$$
\left\{j_{p}(\xi) j_{q}(\eta)^{*} \mid p q^{-1}=g, \xi \in \mathcal{E}_{p} \text { and } \eta \in \mathcal{E}_{q}\right\}
$$

This completes the proof.

Corollary 4.2.15. Let $\mathcal{E}=\left(\mathcal{E}_{p}\right)_{p \in P}$ be a compactly aligned product system and $\mathcal{J}$ as above. Then, for all $p \in P$, we have an isomorphism

$$
\mathcal{O}_{\mathcal{J}, \mathcal{E}}^{p} \cong \mathcal{E}_{p} \otimes_{A} \mathcal{O}_{\mathcal{J}, \mathcal{E}}^{e}
$$

of correspondences $A \leadsto \mathcal{O}_{\mathcal{J}, \mathcal{E}}^{e}$. Moreover, $\left(\mathcal{O}_{\mathcal{J}, \mathcal{E}}^{p}\right)_{p \in P}$ is a product system of Hilbert $\mathcal{O}_{\mathcal{J}, \mathcal{E}^{-b i m o d u l e s .}}^{e}$.

Proof. By Proposition 4.2.14 $\mathcal{O}_{\mathcal{J}, \mathcal{E}}^{p}$ is generated by elements of the form $j_{r}(\xi) j_{s}(\eta)^{*}$, with $\xi \in \mathcal{E}_{r}, \eta \in \mathcal{E}_{s}$ and $r s^{-1}=p$. In particular, $r=p s$ and we can use the isomorphism $\mu_{p, s}^{-1}$ to show that $j_{r}(\xi) j_{s}(\eta)^{*}$ lies in $j_{p}\left(\mathcal{E}_{p}\right) j_{s}\left(\mathcal{E}_{s}\right) j_{s}\left(\mathcal{E}_{s}\right)^{*}$, which in turn is contained in $j_{p}\left(\mathcal{E}_{p}\right) \mathcal{O}_{\mathcal{J}, \mathcal{E}}^{e}$. The inclusion $j_{p}\left(\mathcal{E}_{p}\right) \mathcal{O}_{\mathcal{J}, \mathcal{E}}^{e} \subseteq \mathcal{O}_{\mathcal{J}, \mathcal{E}}^{p}$ is trivial. So $\mathcal{O}_{\mathcal{J}, \mathcal{E}}^{p}=j_{p}\left(\mathcal{E}_{p}\right) \mathcal{O}_{\mathcal{J}, \mathcal{E}}^{e}$. Hence $\mathcal{E}_{p} \otimes_{A} \mathcal{O}_{\mathcal{J}, \mathcal{E}}^{e} \rightarrow \mathcal{O}_{\mathcal{J}, \mathcal{E}}^{p}, \xi \otimes \eta \mapsto j_{p}(\xi) \eta$ gives an isomorphism of correspondences $A \leadsto \mathcal{O}_{\mathcal{J}, \mathcal{E}}^{e}$.

For each $p \in P, \mathcal{O}_{\mathcal{J}, \mathcal{E}}^{p}$ is a Hilbert $\mathcal{O}_{\mathcal{J}, \mathcal{E}^{-}}^{e}$-bimodule with the structure obtained from the multiplication and involution operations on $\mathcal{O}_{\mathcal{J}, \mathcal{E}}$. In particular, $\mathcal{O}_{\mathcal{J}, \mathcal{E}}^{e} \mathcal{O}_{\mathcal{J}, \mathcal{E}}^{p}=\mathcal{O}_{\mathcal{J}, \mathcal{E}}^{p}$. Hence, if $p, q \in P$, we have a correspondence isomorphism

$$
\begin{aligned}
\mathcal{O}_{\mathcal{J}, \mathcal{E}}^{p} \otimes_{\mathcal{O}_{\mathcal{J}, \mathcal{E}}^{e}} \mathcal{O}_{\mathcal{J}, \mathcal{E}}^{q} & \cong \mathcal{O}_{\mathcal{J}, \mathcal{E}}^{p} \mathcal{O}_{\mathcal{J}, \mathcal{E}}^{q}=\left(j_{p}\left(\mathcal{E}_{p}\right) \mathcal{O}_{\mathcal{J}, \mathcal{E}}^{e}\right) \mathcal{O}_{\mathcal{J}, \mathcal{E}}^{q}=j_{p}\left(\mathcal{E}_{p}\right)\left(\mathcal{O}_{\mathcal{J}, \mathcal{E}}^{e} \mathcal{O}_{\mathcal{J}, \mathcal{E}}^{q}\right) \\
& =j_{p}\left(\mathcal{E}_{p}\right) \mathcal{O}_{\mathcal{J}, \mathcal{E}}^{q}=j_{p}\left(\mathcal{E}_{p}\right) j_{q}\left(\mathcal{E}_{q}\right) \mathcal{O}_{\mathcal{J}, \mathcal{E}}^{e}=j_{p q}\left(\mathcal{E}_{p q}\right) \mathcal{O}_{\mathcal{J}, \mathcal{E}}^{e}=\mathcal{O}_{\mathcal{E}, \mathcal{J}}^{p q} .
\end{aligned}
$$

These multiplication maps are associative because they coincide with the multiplication on $\mathcal{O}_{\mathcal{J}, \mathcal{E}}$.

\subsection{Bicategories of compactly aligned product systems}

We define covariant correspondences between compactly aligned product systems as in Chapter 3 . following the ideas of Schweizer [54]. Let $(G, P)$ be a quasi-lattice ordered group. Let $\mathcal{E}=\left(\mathcal{E}_{p}\right)_{p \in P}$ and $\mathcal{G}=\left(\mathcal{G}_{p}\right)_{p \in P}$ be compactly aligned product systems of correspondences over $\mathrm{C}^{*}$-algebras $A$ and $B$, respectively. Let $\mathcal{J}_{A}=\left\{J_{p}^{A}\right\}_{p \in P}$ and $\mathcal{J}_{B}=\left\{J_{p}^{B}\right\}_{p \in P}$ be families of ideals in $A$ and $B$, with $\varphi_{p}^{A}\left(J_{p}^{A}\right) \subseteq \mathbb{K}\left(\mathcal{E}_{p}\right)$ and $\varphi_{p}^{B}\left(J_{p}^{B}\right) \subseteq \mathbb{K}\left(\mathcal{G}_{p}\right)$ for all $p \in P$.

Definition 4.3.1. A covariant correspondence from $\left(A, \mathcal{E}, \mathcal{J}_{A}\right)$ to $\left(B, \mathcal{G}, \mathcal{J}_{B}\right)$ is a pair $(\mathcal{F}, V)$, where $\mathcal{F}: A \leadsto B$ is a correspondence such that $J_{p}^{A} \mathcal{F} \subseteq \mathcal{F} J_{p}^{B}$ for all $p \in P$ and $V=\left\{V_{p}\right\}_{p \in P}$ is a family of correspondence isomorphisms $V_{p}: \mathcal{E}_{p} \otimes_{A} \mathcal{F} \cong \mathcal{F} \otimes_{B} \mathcal{G}_{p}$, where $V_{e}: A \otimes_{A} \mathcal{F} \cong \mathcal{F} \otimes_{B} B$ is the isomorphism 
which sends $a \otimes(\xi b)$ to $\psi(a) \xi \otimes b$. These must make the following diagrams commute for all $p, q \in P$ :

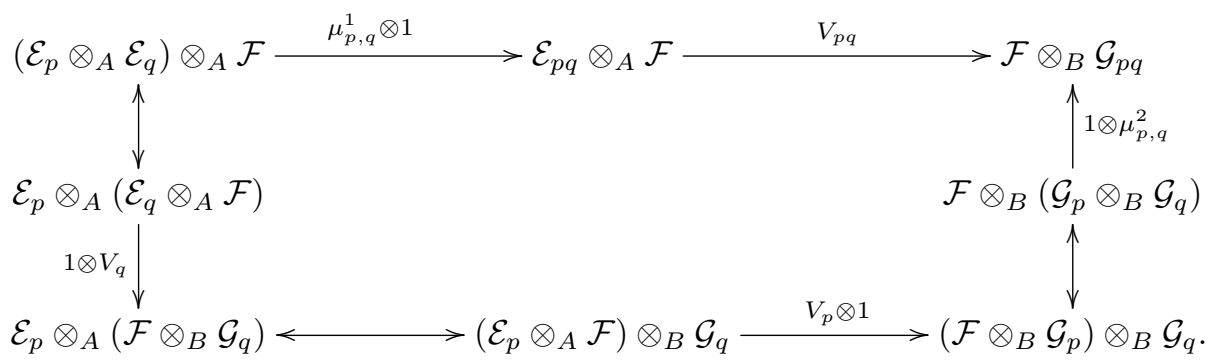

A covariant correspondence $(\mathcal{F}, V)$ is called proper if $\mathcal{F}$ is a proper correspondence.

Definition 4.3.3. The bicategory $\mathfrak{C}^{P}$ has the following data:

- Objects are triples $(A, \mathcal{E}, \mathcal{J})$, where $A$ is a $\mathrm{C}^{*}$-algebra, $\mathcal{E}=\left(\mathcal{E}_{p}\right)_{p \in P}$ is a compactly aligned product system over $P$ of $A$-correspondences, and $\mathcal{J}=\left\{J_{p}\right\}_{p \in P}$ is a family of ideals in $A$ with $J_{p} \subseteq \varphi_{p}^{-1}\left(\mathbb{K}\left(\mathcal{E}_{p}\right)\right)$ for all $p \in P$.

- Arrows $(A, \mathcal{E}, \mathcal{J}) \rightarrow\left(A_{1}, \mathcal{E}_{1}, \mathcal{J}_{1}\right)$ are covariant correspondences $(\mathcal{F}, V)$ from $(A, \mathcal{E}, \mathcal{J})$ to $\left(A_{1}, \mathcal{E}_{1}, \mathcal{J}_{1}\right)$.

- 2-Arrows $\left(\mathcal{F}_{0}, V_{0}\right) \Rightarrow\left(\mathcal{F}_{1}, V_{1}\right)$ are isomorphisms of covariant correspondences, that is, correspondence isomorphisms $w: \mathcal{F}_{0} \rightarrow \mathcal{F}_{1}$ for which the following diagram commutes for all $p \in P$ :

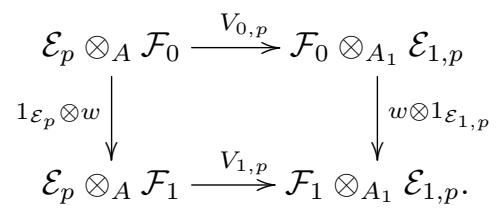

- The vertical product of 2-arrows

$$
w_{0}:\left(\mathcal{F}_{0}, V_{0}\right) \Rightarrow\left(\mathcal{F}_{1}, V_{1}\right), \quad w_{1}:\left(\mathcal{F}_{1}, V_{1}\right) \Rightarrow\left(\mathcal{F}_{2}, V_{2}\right)
$$

is the usual product $w_{1} \cdot w_{0}: \mathcal{F}_{0} \rightarrow \mathcal{F}_{2}$. The arrows $(A, \mathcal{E}, \mathcal{J}) \rightarrow\left(A_{1}, \mathcal{E}_{1}, \mathcal{J}_{1}\right)$ and the 2-arrows between them form a groupoid $\mathfrak{C}^{P}\left((A, \mathcal{E}, \mathcal{J}),\left(A_{1}, \mathcal{E}_{1}, \mathcal{J}_{1}\right)\right)$.

- Let $(\mathcal{F}, V):(A, \mathcal{E}, \mathcal{J}) \rightarrow\left(A_{1}, \mathcal{E}_{1}, \mathcal{J}_{1}\right)$ and $\left(\mathcal{F}_{1}, V_{1}\right):\left(A_{1}, \mathcal{E}_{1}, \mathcal{J}_{1}\right) \rightarrow\left(A_{2}, \mathcal{E}_{2}, \mathcal{J}_{2}\right)$ be arrows. For each $p \in P$, let $V_{p} \bullet V_{1, p}$ be the composite correspondence isomorphism

$$
\mathcal{E}_{p} \otimes_{A} \mathcal{F} \otimes_{A_{1}} \mathcal{F}_{1} \stackrel{V_{p} \otimes 1_{\mathcal{F}_{1}}}{\longrightarrow} \mathcal{F} \otimes_{A_{1}} \mathcal{E}_{1, p} \otimes_{A_{1}} \mathcal{F}_{1} \stackrel{1_{\mathcal{F}} \otimes V_{1, p}}{\longrightarrow} \mathcal{F} \otimes_{A_{1}} \mathcal{F}_{1} \otimes_{A_{2}} \mathcal{E}_{2, p}
$$

We define the product $\left(\mathcal{F}_{1}, V_{1}\right) \circ(\mathcal{F}, V)$ by

$$
\left(\mathcal{F}_{1}, V_{1}\right) \circ(\mathcal{F}, V):=\left(\mathcal{F} \otimes_{A_{1}} \mathcal{F}_{1}, V \bullet V_{1}\right),
$$

where $V \bullet V_{1}=\left\{V_{p} \bullet V_{1, p}\right\}_{p \in P}$.

- The horizontal product for a diagram of arrows and 2-arrows

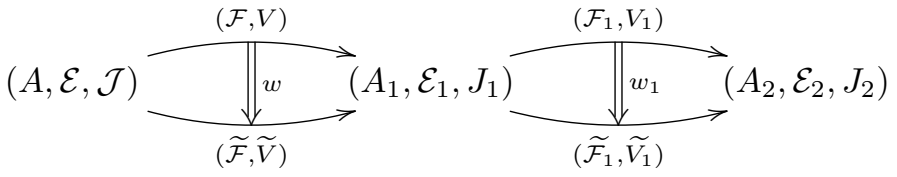

is the 2 -arrow

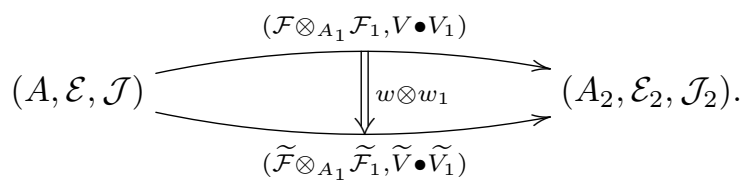




\section{PRODUCT SYSTEMS AND SOME C*-CONSTRUCTIONS}

This horizontal product and the product of arrows produce composition bifunctors

$$
\mathfrak{C}^{P}\left((A, \mathcal{E}, \mathcal{J}),\left(A_{1}, \mathcal{E}_{1}, \mathcal{J}_{1}\right)\right) \times \mathfrak{C}^{P}\left(\left(A_{1}, \mathcal{E}_{1}, \mathcal{J}_{1}\right),\left(A_{2}, \mathcal{E}_{2}, \mathcal{J}_{2}\right)\right) \rightarrow \mathfrak{C}^{P}\left((A, \mathcal{E}, \mathcal{J}),\left(A_{2}, \mathcal{E}_{2}, \mathcal{J}_{2}\right)\right) .
$$

- The unit arrow on the object $(A, \mathcal{E}, \mathcal{J})$ is the proper covariant correspondence $\left(A, \iota_{\mathcal{E}}\right)$, where $A$ is the identity correspondence and $\iota_{\mathcal{E}}=\left\{\iota_{\mathcal{E}}\right\}_{p \in P}$ is the family of canonical isomorphisms

$$
\mathcal{E}_{p} \otimes_{A} A \cong \mathcal{E}_{p} \cong A \otimes_{A} \mathcal{E}_{p}
$$

obtained from the right and left actions of $A$ on $\mathcal{E}$.

- The associators and unitors are the same as in the correspondence bicategory.

We will denote by $\mathfrak{C}_{\mathrm{pr}}^{P}$ the sub-bicategory of $\mathfrak{C}^{P}$ whose arrows are proper covariant correspondences. Example 4.3.4. View $\mathcal{O}_{\mathcal{J}, \mathcal{E}}^{e}$ as a correspondence $A \sim \mathcal{O}_{\mathcal{J}, \mathcal{E}}^{e}$. For each $p \in P$, let $\hat{\iota}_{\mathcal{E}_{p}}$ be the isomorphism

$$
\mathcal{E}_{p} \otimes_{A} \mathcal{O}_{\mathcal{J}, \mathcal{E}}^{e} \cong \mathcal{O}_{\mathcal{J}, \mathcal{E}}^{p} \cong \mathcal{O}_{\mathcal{J}, \mathcal{E}}^{e} \otimes_{\mathcal{O}_{\mathcal{J}, \mathcal{E}}^{e}} \mathcal{O}_{\mathcal{J}, \mathcal{E}}^{p}
$$

where the first isomorphism is that of Corollary 4.2.15. Cuntz-Pimsner covariance on $\mathcal{J}=\left\{J_{p}\right\}_{p \in P}$ implies that

$$
j_{e}\left(J_{p}\right) \subseteq j_{p}\left(\mathcal{E}_{p}\right) j_{p}\left(\mathcal{E}_{p}\right)^{*} \subseteq \mathcal{O}_{\mathcal{J}, \mathcal{E}}^{p} \mathcal{O}_{\mathcal{J}, \mathcal{E}}^{p *}
$$

for all $p \in P$. So $\left(\mathcal{O}_{\mathcal{J}, \mathcal{E}}^{e}, \hat{\iota}_{\mathcal{E}}\right)$ is a proper covariant correspondence

$$
(A, \mathcal{E}, \mathcal{J}) \rightarrow\left(\mathcal{O}_{\mathcal{J}, \mathcal{E}}^{e},\left(\mathcal{O}_{\mathcal{J}, \mathcal{E}}^{p}\right)_{p \in P}, \mathcal{I}_{\mathcal{O}_{\mathcal{J}, \mathcal{E}}}\right)
$$

where $\hat{\iota}_{\mathcal{E}}=\left\{\hat{\iota}_{\mathcal{E}_{p}}\right\}_{p \in P}$ and $\mathcal{I}_{\mathcal{O}_{\mathcal{J}, \mathcal{E}}}=\left\{I_{p}^{\mathcal{O}_{\mathcal{J}, \mathcal{E}}}\right\}_{p \in P}$ with $I_{p}^{\mathcal{O}_{\mathcal{J}, \mathcal{E}}}=\mathcal{O}_{\mathcal{J}, \mathcal{E}}^{p} \mathcal{O}_{\mathcal{J}, \mathcal{E}}^{p *}=\left\langle\left\langle\mathcal{O}_{\mathcal{J}, \mathcal{E}}^{p} \mid \mathcal{O}_{\mathcal{I} \mathcal{E}}^{p}\right\rangle\right\rangle$

We will see in the sequel that $\left(\mathcal{O}_{\mathcal{J}, \mathcal{E}}^{e}, \hat{\iota}_{\mathcal{E}}\right)$ is a universal arrow as in Definition 3.3.2. So the triple $\left(\mathcal{O}_{\mathcal{J}, \mathcal{E}}^{e},\left(\mathcal{O}_{\mathcal{J}, \mathcal{E}}^{p}\right)_{p \in P}, \mathcal{I}_{\mathcal{O}_{\mathcal{J}, \mathcal{E}}}\right)$ is the best approximation of $(A, \mathcal{E}, \mathcal{J})$ by an object of a certain sub-bicategory of $\mathfrak{C}_{\mathrm{pr}}^{P}$ of product systems of Hilbert bimodules. 


\section{Chapter 5}

\section{Fell bundles over quasi-lattice ordered groups}

In this chapter we introduce a class of Fell bundles over quasi-lattice ordered groups, inspired by properties of the topological grading of relative Cuntz-Pimsner algebras. We show that such Fell bundles arise naturally from a certain class of product systems of Hilbert bimodules. This correspondence turns out to be an equivalence between a sub-bicategory of $\mathfrak{C}^{P}$ and a bicategory of Fell bundles. In Section 5.3 we relate amenability for this class of Fell bundles to amenability of quasi-lattice ordered groups by means of examples.

With the above equivalence at hand, we generalise the results from Chapter 3 for relative CuntzPimsner algebras of single correspondences to those of compactly aligned product systems. This will be done in Section 5.4 We apply this to study Morita equivalence of relative Cuntz-Pimsner algebras.

\subsection{From product systems of Hilbert bimodules to Fell bun- dles}

Definition 5.1.1. Let $(G, P)$ be a quasi-lattice ordered group and let $\left(B_{g}\right)_{g \in G}$ be a Fell bundle over $G$. We will say that $\left(B_{g}\right)_{g \in G}$ is semi-saturated with respect to the quasi-lattice ordered group structure of $(G, P)$ if it satisfies the following conditions:

(S1) $B_{p} B_{q}=B_{p q}$ for all $p, q \in P$;

(S2) $B_{g}=B_{(g \vee e)} B_{\left(g^{-1} \vee e\right)}^{*}$ for all $g \in G$ with $g \vee e<\infty$;

Definition 5.1.2. A Fell bundle over $G$ will be called orthogonal with respect to $(G, P)$ if $B_{g}=\{0\}$ whenever $g \vee e=\infty$.

Let $\mathbb{F}$ be the free group on a set of generators $S$. A Fell bundle over $\mathbb{F}$ is semi-saturated in the sense of Exel if $B_{g} B_{h}=B_{g h}$ for all $g, h \in \mathbb{F}$ such that the multiplication $g \cdot h$ involves no cancellation. It is called orthogonal if $B_{s}^{*} B_{t}=\{0\}$ whenever $s$ and $t$ are distinct generators of $\mathbb{F}$ (see $[19$ for further details). Let $\mathbb{F}^{+}$be the unital subsemigroup of $\mathbb{F}$ generated by $S$. Recall from Example 4.2 .3 that $\left(\mathbb{F}, \mathbb{F}^{+}\right)$is a quasi-lattice ordered group and that an element $g \in \mathbb{F}$ satisfies $g \vee e<\infty$ if and only if its reduced form is $p q^{-1}$, with $p, q$ in $P$. In this case, $g \vee e=p$ and $g^{-1} \vee e=q$. The following result compares our definitions of semi-saturatedness and orthogonality for Fell bundles over $\mathbb{F}$ with those introduced by Exel.

Proposition 5.1.3. A Fell bundle $\left(B_{g}\right)_{g \in \mathbb{F}}$ is semi-saturated and orthogonal with respect to $\left(\mathbb{F}, \mathbb{F}^{+}\right)$if and only if it is both semi-saturated and orthogonal as defined in [19].

Proof. Suppose that $\left(B_{g}\right)_{g \in \mathbb{F}}$ is semi-saturated and orthogonal with respect to $\left(\mathbb{F}, \mathbb{F}^{+}\right)$. Then orthogonality implies that $\left(B_{g}\right)_{g \in \mathbb{F}}$ is orthogonal as defined by Exel, since $\left(p^{-1} q\right) \vee e=\infty$ if $p$ and $q$ are distinct generators of $\mathbb{F}$. In order to prove that $\left(B_{g}\right)_{g \in \mathbb{F}}$ is also semi-saturated according to $\sqrt{19}$, let $g, h \in \mathbb{F}$ be such that the product $g \cdot h$ involves no cancellation. If $g h \vee e=\infty$, then $B_{g h}=\{0\}=B_{g} B_{h}$. Assume that $(g h) \vee e<\infty$. First, this implies that either $g$ belongs to $\mathbb{F}^{+}$and $h \vee e<\infty$ or $g \vee e<\infty$ 


\section{FELL BUNDLES OVER QUASI-LATTICE ORDERED GROUPS}

and $h \in\left(\mathbb{F}^{+}\right)^{-1}$ because $g h$ has reduced form $p q^{-1}$ with $p, q \in P$ and the product $g \cdot h$ involves no cancellation. In case $g \in \mathbb{F}^{+}$, we then have $g(h \vee e)=g h \vee e$ and $(g h)^{-1} \vee e=\left(h^{-1} g^{-1}\right) \vee e=h^{-1} \vee e$. So axioms (S1) and (S2) give us

$$
B_{g} B_{h}=B_{g} B_{h \vee e} B_{h^{-1} \vee e}^{*}=B_{g(h \vee e)} B_{h^{-1} \vee e}^{*}=B_{(g h) \vee e} B_{(g h)^{-1} \vee e}^{*}=B_{g h} .
$$

Now if $h \in\left(\mathbb{F}^{+}\right)^{-1}$, it follows from the previous case that

$$
B_{g} B_{h}=\left(B_{h^{-1}} B_{g^{-1}}\right)^{*}=B_{h^{-1} g^{-1}}^{*}=B_{g h} .
$$

This shows that $\left(B_{g}\right)_{g \in \mathbb{F}}$ is semi-saturated as defined in $[19$.

Now suppose that $\left(B_{g}\right)_{g \in \mathbb{F}}$ is a Fell bundle that is semi-saturated and orthogonal according to 19. Clearly, $\left(B_{g}\right)_{g \in \mathbb{F}}$ satisfies (S1). Any element of $\mathbb{F}$ has a reduced form, so that orthogonality as in Definition 5.1 .2 follows by combining semi-saturatedness and orthogonality of $\left(B_{g}\right)_{g \in \mathbb{F}}$. Given $g \in \mathbb{F}$ with $g \vee e<\infty$, the product $(g \vee e)\left(g^{-1} \vee e\right)^{-1}$ involves no cancellation. Therefore, semi-saturatedness gives us

$$
B_{g}=B_{g \vee e} B_{\left(g^{-1} \vee e\right)^{-1}}=B_{g \vee e} B_{\left(g^{-1} \vee e\right)}^{*} .
$$

This completes the proof of the statement.

Our main examples of Fell bundles that are semi-saturated and orthogonal come from the grading of relative Cuntz-Pimsner algebras associated to compactly aligned product systems obtained in Proposition 4.2.14 In fact, we will prove that any Fell bundle that is semi-saturated and orthogonal is isomorphic to one of this form.

Example 5.1.4. Let $\mathcal{E}=\left(\mathcal{E}_{p}\right)_{p \in P}$ be a compactly aligned product system and let $\mathcal{J}=\left\{J_{p}\right\}_{p \in P}$ be a family of ideals in $A$ with $J_{p} \subseteq \varphi_{p}^{-1}\left(\mathbb{K}\left(\mathcal{E}_{p}\right)\right)$ for all $p \in P$. Then $\left(\mathcal{O}_{\mathcal{J}, \mathcal{E}}^{g}\right)_{g \in G}$ is orthogonal because $\mathcal{O}_{\mathcal{J}, \mathcal{E}}^{g}=\{0\}$ whenever $g \vee e=\infty$. To see that it is also semi-saturated, observe that if $p, q \in P$ satisfy $p q^{-1}=g$, then there is $r \in P$ with $p=(g \vee e) r$ and $q=\left(g^{-1} \vee e\right) r$. Indeed, since $g \vee e$ and $g^{-1} \vee e$ are the least upper bounds for $g$ and $g^{-1}$ in $P$, respectively, there are $r, s \in P$ such that $p=(g \vee e) r$ and $q=\left(g^{-1} \vee e\right) s$. The equality $g=(g \vee e)\left(g^{-1} \vee e\right)^{-1}=(g \vee e) r s^{-1}\left(g^{-1} \vee e\right)^{-1}$ entails $r=s$.

Thus, given $g$ in $G$ with $g \vee e<\infty$, write $g=(g \vee e)\left(g^{-1} \vee e\right)^{-1}$. By Proposition 4.2.14, $\mathcal{O}_{\mathcal{J}, \mathcal{E}}^{g}$ is spanned by the elements of the form $j_{p}(\xi) j_{q}(\eta)^{*}$, with $\xi \in \mathcal{E}_{p}, \eta \in \mathcal{E}_{q}$ and $p q^{-1}=g$. Given such an element $j_{p}(\xi) j_{q}(\eta)^{*}$, let $r \in P$ be such that $p=(g \vee e) r$ and $q=\left(g^{-1} \vee e\right) r$. We then employ the isomorphisms $\mu_{g \vee e, r}^{-1}$ and $\mu_{g^{-1} \vee e, r}^{-1}$ to conclude that

$$
j_{p}(\xi) j_{q}(\eta)^{*} \in j_{g \vee e}\left(\mathcal{E}_{g \vee e}\right) j_{r}\left(\mathcal{E}_{r}\right) j_{r}\left(\mathcal{E}_{r}\right)^{*} j_{g^{-1} \vee e}\left(\mathcal{E}_{g^{-1} \vee e}\right)^{*} \subseteq \mathcal{O}_{\mathcal{J}, \mathcal{E}}^{g \vee e}\left(\mathcal{O}_{\mathcal{J}, \mathcal{E}}^{g^{-1} \vee e}\right)^{*}
$$

Therefore, $\left(\mathcal{O}_{\mathcal{J}, \mathcal{E}}^{g}\right)_{g \in G}$ satisfies $(\mathrm{S} 2)$. Now axiom (S1) follows from Corollary 4.2.15 Thus $\left(\mathcal{O}_{\mathcal{J}, \mathcal{E}}^{g}\right)_{g \in G}$ is also semi-saturated.

Definition 5.1.5. A product system of Hilbert bimodules $\mathcal{E}=\left(\mathcal{E}_{p}\right)_{p \in P}$ will be called simplifiable if for all $p, q \in P$ one has

(i) $\left\langle\left\langle\mathcal{E}_{p} \mid \mathcal{E}_{p}\right\rangle\right\rangle\left\langle\left\langle\mathcal{E}_{q} \mid \mathcal{E}_{q}\right\rangle\right\rangle \subseteq\left\langle\left\langle\mathcal{E}_{p \vee q} \mid \mathcal{E}_{p \vee q}\right\rangle\right\rangle \quad$ if $p \vee q<\infty$;

(ii) $\left\langle\left\langle\mathcal{E}_{p} \mid \mathcal{E}_{p}\right\rangle\right\rangle\left\langle\left\langle\mathcal{E}_{q} \mid \mathcal{E}_{q}\right\rangle\right\rangle=\{0\} \quad$ if $p \vee q=\infty$;

here $\langle\langle\cdot \mid \cdot\rangle\rangle$ denotes the left $A$-valued inner product.

Remark 5.1.6. A simplifiable product system of Hilbert bimodules is compactly aligned. The converse is not true in general. For instance, take a nontrivial Hilbert bimodule $\mathcal{E}$ over a $\mathrm{C}^{*}$-algebra $A$ satisfying $\mathcal{E} \otimes_{A} \mathcal{E}=\{0\}$. This produces a product system over $\mathbb{N} \times \mathbb{N}$ such that $\mathcal{E}_{(1,0)}=\mathcal{E}_{(0,1)}=\mathcal{E}$. It is compactly aligned because $\mathcal{E}_{(1,1)}=\{0\}$, but $\left\langle\left\langle\mathcal{E}_{(1,0)} \mid \mathcal{E}_{(1,0)}\right\rangle\right\rangle=\left\langle\left\langle\mathcal{E}_{(0,1)} \mid \mathcal{E}_{(0,1)}\right\rangle\right\rangle \neq\{0\}$.

Proposition 5.1.7. Let $\mathcal{E}=\left(\mathcal{E}_{p}\right)_{p \in P}$ be a simplifiable product system of Hilbert bimodules. For each $p \in P$, let $I_{p}:=\left\langle\left\langle\mathcal{E}_{p} \mid \mathcal{E}_{p}\right\rangle\right\rangle$ and set $\mathcal{I}=\left\{I_{p}\right\}_{p \in P}$. If $\psi=\left\{\psi_{p}\right\}_{p \in P}$ is a representation of $\mathcal{E}$ in a $\mathrm{C}^{*}$-algebra $B$ that is Cuntz-Pimsner covariant on $\mathcal{I}$, then it is also Nica covariant. 
Proof. Let $p, q \in P, T \in \mathbb{K}\left(\mathcal{E}_{p}\right)$ and $S \in \mathbb{K}\left(\mathcal{E}_{q}\right)$. Let $a \in I_{p}$ and $b \in I_{q}$ be such that $\varphi_{p}(a)=T$ and $\varphi_{q}(b)=S$. Cuntz-Pimsner covariance on $\mathcal{I}$ gives us

$$
\psi^{(p)}(T) \psi^{(q)}(S)=\psi_{e}(a) \psi_{e}(b)=\psi_{e}(a b)
$$

So by condition (ii) of Definition 5.1.5, $\psi^{(p)}(T) \psi^{(q)}(S)=0$ if $p \vee q=\infty$. In case $p \vee q<\infty$, it follows that $\iota_{p}^{p \vee q}(T) \iota_{q}^{p \vee q}(S)=\varphi_{p \vee q}(a b)$. Applying the Cuntz-Pimsner covariance condition to $a b \in I_{p \vee q}$, we obtain

$$
\psi^{(p)}(T) \psi^{(q)}(S)=\psi_{e}(a b)=\psi^{(p \vee q)}\left(\varphi_{p \vee q}(a b)\right)=\psi^{(p \vee q)}\left(\iota_{p}^{p \vee q}(T) \iota_{q}^{p \vee q}(S)\right) .
$$

Therefore, $\psi$ is Nica covariant.

Theorem 5.1.8. Let $(G, P)$ be a quasi-lattice ordered group and let $\mathcal{E}=\left(\mathcal{E}_{p}\right)_{p \in P}$ be a simplifiable product system of Hilbert bimodules. There is a semi-saturated and orthogonal Fell bundle $\hat{\mathcal{E}}=\left(\hat{\mathcal{E}}_{g}\right)_{g \in G}$ extending the structure of product system of $\mathcal{E}$, in the sense that

(i) there are isomorphisms $j_{p}: \mathcal{E}_{p} \cong \hat{\mathcal{E}}_{p}$ of complex vector spaces such that $j_{e}: A \rightarrow \hat{\mathcal{E}}_{e}$ is a ${ }^{*}$-isomorphism and $j_{p}(\xi) j_{q}(\eta)=j_{p q}\left(\mu_{p, q}(\xi \otimes \eta)\right)$ for all $p, q \in P$;

(ii) $j_{p}(\xi)^{*} j_{p}(\eta)=j_{e}(\langle\xi \mid \eta\rangle)$ for all $\xi, \eta \in \mathcal{E}_{p}$ and $p \in P$, where ${ }^{*}: \hat{\mathcal{E}}_{p} \rightarrow \hat{\mathcal{E}}_{p^{-1}}$ is the involution operation on $\hat{\mathcal{E}}$.

Moreover, $\hat{\mathcal{E}}$ is unique up to canonical isomorphism of Fell bundles.

Proof. We begin by building the fibres of such a Fell bundle. For each $g \in G$, we set

$$
\hat{\mathcal{E}}_{g}:= \begin{cases}\mathcal{E}_{g \vee e} \otimes_{A} \mathcal{E}_{g^{-1} \vee e}^{*} & \text { if } g \vee e<\infty, \\ \{0\} & \text { otherwise }\end{cases}
$$

Here $\mathcal{E}_{g^{-1} \vee e}$ is the adjoint Hilbert bimodule of $\mathcal{E}_{g^{-1} \vee e}$ (see Proposition A.2.7). We may identify $\hat{\mathcal{E}}_{p}$ with $\mathcal{E}_{p}$ and $\hat{\mathcal{E}}_{p^{-1}}$ with $\mathcal{E}_{p}^{*}$ by using the canonical isomorphisms $A \otimes_{A} \mathcal{E}_{p}^{*} \cong \mathcal{E}_{p}^{*}$ and $\mathcal{E}_{p} \otimes_{A} A^{*} \cong \mathcal{E}_{p}$, where $A$ is identified with $A^{*}$ through $a \mapsto \widetilde{a^{*}}$. So we will omit the map $j_{p}: \mathcal{E}_{p} \rightarrow \hat{\mathcal{E}}_{p}$ mentioned in the statement.

If $p, q \in P$ satisfy $p q^{-1}=g$, then there is $r \in P$ with $p=(g \vee e) r$ and $q=\left(g^{-1} \vee e\right) r$ (see this computation in Example 5.1.4. Hence we may view $\mathcal{E}_{p} \otimes_{A} \mathcal{E}_{q}^{*}$ as a sub-bimodule of $\mathcal{E}_{g \vee e} \otimes_{A} \mathcal{E}_{g^{-1} \vee e}^{*}$ through the embedding

$$
\begin{aligned}
\mathcal{E}_{p} \otimes_{A} \mathcal{E}_{q}^{*} & \cong \mathcal{E}_{g \vee e} \otimes_{A} \mathcal{E}_{r} \otimes_{A} \mathcal{E}_{r}^{*} \otimes_{A} \mathcal{E}_{g^{-1} \vee e}^{*} \\
& \cong \mathcal{E}_{g \vee e}\left\langle\left\langle\mathcal{E}_{r} \mid \mathcal{E}_{r}\right\rangle\right\rangle \otimes_{A} \mathcal{E}_{g^{-1} \vee e} \\
& \hookrightarrow \mathcal{E}_{g \vee e} \otimes_{A} \mathcal{E}_{g^{-1} \vee e}^{*}
\end{aligned}
$$

We will use this inclusion and the fact that $\mathcal{E}$ is simplifiable to define the multiplication maps $\hat{\mu}_{g, h}: \hat{\mathcal{E}}_{g} \times$ $\hat{\mathcal{E}}_{h} \rightarrow \hat{\mathcal{E}}_{g h}$. Let $p q^{-1}$ and $r s^{-1}$ be the reduced forms for $g$ and $h$, respectively. The canonical isomorphisms

$$
\begin{aligned}
& \mathcal{E}_{q}^{*} \otimes_{A} \mathcal{E}_{q} \cong\left\langle\mathcal{E}_{q} \mid \mathcal{E}_{q}\right\rangle, \\
& \mathcal{E}_{r}^{*} \otimes_{A} \mathcal{E}_{r} \cong\left\langle\mathcal{E}_{r} \mid \mathcal{E}_{r}\right\rangle, \\
& \mathcal{E}_{q} \otimes_{A} \mathcal{E}_{q}^{*} \cong\left\langle\left\langle\mathcal{E}_{q} \mid \mathcal{E}_{q}\right\rangle\right\rangle, \\
& \mathcal{E}_{r} \otimes_{A} \mathcal{E}_{r}^{*} \cong\left\langle\left\langle\mathcal{E}_{r} \mid \mathcal{E}_{r}\right\rangle\right\rangle,
\end{aligned}
$$

from Proposition 3.1.6, imply

$$
\begin{aligned}
\mathcal{E}_{p} \otimes_{A} \mathcal{E}_{q}^{*} \otimes_{A} \mathcal{E}_{r} \otimes_{A} \mathcal{E}_{s}^{*} & \cong \mathcal{E}_{p} \otimes_{A} \mathcal{E}_{q}^{*} \otimes_{A}\left(\mathcal{E}_{q} \otimes_{A} \mathcal{E}_{q}^{*} \otimes_{A} \mathcal{E}_{r} \otimes_{A} \mathcal{E}_{r}^{*}\right) \otimes_{A} \mathcal{E}_{r} \otimes_{A} \mathcal{E}_{s}^{*} \\
& \hookrightarrow \mathcal{E}_{p} \otimes_{A} \mathcal{E}_{q}^{*} \otimes_{A}\left(\mathcal{E}_{q \vee r} \otimes_{A} \mathcal{E}_{q \vee r}^{*}\right) \otimes_{A} \mathcal{E}_{r} \otimes_{A} \mathcal{E}_{s}^{*} \\
& \cong \mathcal{E}_{p} \otimes_{A}\left\langle\mathcal{E}_{q} \mid \mathcal{E}_{q}\right\rangle \mathcal{E}_{q^{-1}(q \vee r)} \otimes_{A} \mathcal{E}_{r^{-1}(q \vee r)}^{*}\left\langle\mathcal{E}_{r} \mid \mathcal{E}_{r}\right\rangle \otimes_{A} \mathcal{E}_{s}^{*} \\
& \hookrightarrow \mathcal{E}_{p q^{-1}(q \vee r)} \mathcal{E}_{s r^{-1}(q \vee r)}^{*} \hookrightarrow \mathcal{E}_{(g h) \vee e} \otimes_{A} \mathcal{E}_{(g h)^{-1} \vee e}^{*}
\end{aligned}
$$

This yields an isometry $\hat{\mu}_{g, h}: \hat{\mathcal{E}}_{g} \otimes_{A} \hat{\mathcal{E}}_{h} \rightarrow \hat{\mathcal{E}}_{g h}$. We then define the product $\xi_{g} \xi_{h}$ for $\xi_{g} \in \hat{\mathcal{E}}_{g}$ and $\xi_{h} \in \hat{\mathcal{E}}_{h}$ to be the image of $\xi_{g} \otimes_{A} \xi_{h}$ in $\hat{\mathcal{E}}_{g h}$ under $\hat{\mu}_{g, h}$. This satisfies $\hat{\mu}_{p, q}=\mu_{p, q}$ for all $p, q \in P$. The involution 


\section{FELL BUNDLES OVER QUASI-LATTICE ORDERED GROUPS}

${ }^{*}: \hat{\mathcal{E}}_{g} \rightarrow \hat{\mathcal{E}}_{g^{-1}}$ sends an elementary tensor $\xi_{p} \otimes_{A} \eta_{q}^{*}$ to $\eta_{q} \otimes_{A} \xi_{p}^{*}$, where $\xi_{p} \in \mathcal{E}_{p}, \eta_{q} \in \mathcal{E}_{q}$ and $\eta_{q}^{*}$ is the image of $\eta_{q}$ under the canonical conjugate-linear map $\mathcal{E}_{q} \rightarrow \mathcal{E}_{q}^{*}$. Since this latter map is isometric by Corollary A.2.5 the involution is isometric too. Given $\xi \in \mathcal{E}_{p} \otimes \mathcal{E}_{q}^{*}$, the product $\xi^{*} \xi$ coincides with the inner product $\langle\xi \mid \xi\rangle$. Hence it is a positive element in $\hat{\mathcal{E}}_{e}=A$ and $\left\|\xi^{*} \xi\right\|=\|\xi\|^{2}$.

Let us verify the equality $(\xi \eta)^{*}=\eta^{*} \xi^{*}$. To do so, let $p, q, r, s \in P$. First, notice that

$$
\left(\left\langle\left\langle\xi_{q} \mid \xi_{q}^{\prime}\right\rangle\right\rangle\left\langle\left\langle\eta_{r} \mid \eta_{r}^{\prime}\right\rangle\right\rangle\right)^{*}=\left\langle\left\langle\eta_{r} \mid \eta_{r}^{\prime}\right\rangle\right\rangle^{*}\left\langle\left\langle\xi_{q} \mid \xi_{q}^{\prime}\right\rangle\right\rangle^{*}=\left\langle\left\langle\eta_{r}^{\prime} \mid \eta_{r}\right\rangle\right\rangle\left\langle\left\langle\xi_{q}^{\prime} \mid \xi_{q}\right\rangle\right\rangle
$$

For all $p^{\prime}, q^{\prime} \in P, \mu_{p^{\prime}, q^{\prime}}(\xi \otimes \eta)$ is mapped to $\mu_{p^{\prime}, q^{\prime}}(\xi \otimes \eta)^{*}$ through the conjugate-linear map $\mathcal{E}_{p^{\prime} q^{\prime}} \rightarrow \mathcal{E}_{p^{\prime} q^{\prime}}^{*}$. Hence the diagram

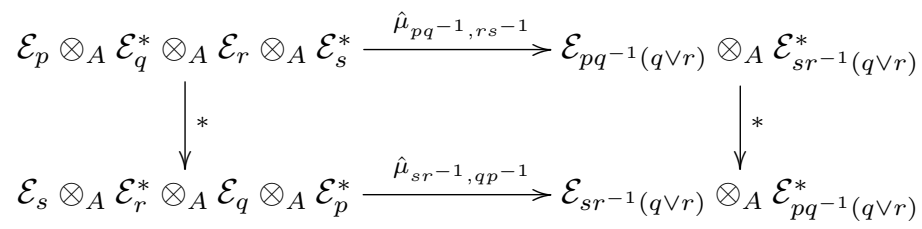

commutes. This shows that $(\xi \eta)^{*}=\eta^{*} \xi^{*}$ for all $\xi \in B_{p q^{-1}}$ and $\eta \in B_{r s^{-1}}$. Now let $\xi \in \mathcal{E}_{p} \otimes_{A} \mathcal{E}_{q}^{*}$ and $\eta \in \mathcal{E}_{r} \otimes_{A} \mathcal{E}_{s}^{*}$. Then

$$
\|\xi \cdot \eta\|^{2}=\|\langle\xi \otimes \eta \mid \xi \otimes \eta\rangle\|=\|\langle\xi \mid\langle\eta \mid \eta\rangle \xi\rangle\| \leq\|\eta\|^{2}\|\xi\|^{2}
$$

and hence $\|\xi \cdot \eta\| \leq\|\xi\|\|\eta\|$.

It remains to check that the multiplication maps are associative. These are associative when restricted to the positive fibres. Hence it suffices to prove that

$$
\hat{\mu}_{g h, k} \circ\left(\hat{\mu}_{g, h} \otimes 1\right)=\hat{\mu}_{g, h k} \circ\left(1 \otimes \hat{\mu}_{h, k}\right)
$$

for $g \in P^{-1}$ and $k \in P$. In addition, for all $p, q, r, s \in P$ with $p \vee q, r \vee s<\infty$, we have that

$$
\begin{aligned}
q r^{-1}\left(\left(r q^{-1}(p \vee q)\right) \vee s\right) & =q r^{-1}\left(\left(r q^{-1}(p \vee q)\right) \vee(r \vee s)\right) \\
& =q\left(q^{-1}(p \vee q) \vee r^{-1}(r \vee s)\right) \\
& =p \vee\left(q r^{-1}(r \vee s)\right) .
\end{aligned}
$$

Thus all we need to show is that the diagram

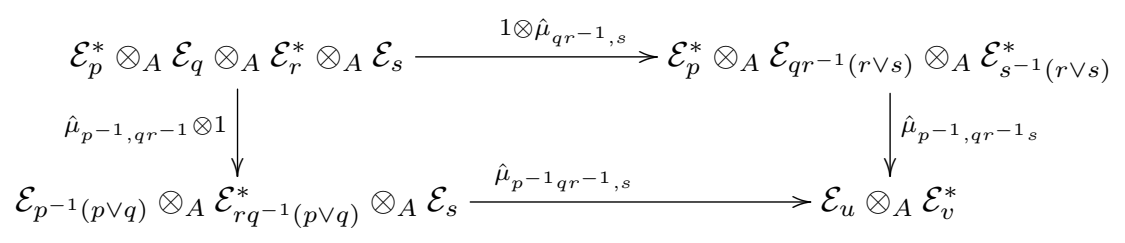

commutes, where

$$
u=p^{-1}\left(q r^{-1}\left(r q^{-1}(p \vee q)\right) \vee s\right)=p^{-1}\left(p \vee\left(q r^{-1}(r \vee s)\right)\right.
$$

and

$$
v=s^{-1}\left(r q^{-1}(p \vee q) \vee s\right)=s^{-1} r q^{-1}\left(p \vee\left(q r^{-1}(r \vee s)\right)\right) .
$$

In what follows, we will identify the ideal $\left\langle\mathcal{E}_{p^{\prime} q^{\prime}} \mid \mathcal{E}_{p^{\prime} q^{\prime}}\right\rangle$ with $\left\langle\mathcal{E}_{q^{\prime}} \mid\left\langle\mathcal{E}_{p^{\prime}} \mid \mathcal{E}_{p^{\prime}}\right\rangle \mathcal{E}_{q^{\prime}}\right\rangle$ via $\mu_{p^{\prime}, q^{\prime}}^{-1}$. We also identify $\left\langle\left\langle\mathcal{E}_{p^{\prime} q^{\prime}} \mid \mathcal{E}_{p^{\prime} q^{\prime}}\right\rangle\right\rangle$ with $\left\langle\left\langle\mathcal{E}_{p^{\prime}}\left\langle\left\langle\mathcal{E}_{q^{\prime}} \mid \mathcal{E}_{q^{\prime}}\right\rangle\right\rangle \mid \mathcal{E}_{p^{\prime}}\right\rangle\right\rangle$ in the same way. Since $\mathcal{E}$ is simplifiable, it follows that $\left\langle\left\langle\mathcal{E}_{p} \mid \mathcal{E}_{p}\right\rangle\right\rangle$ maps $\mathcal{E}_{q}$ into $\mathcal{E}_{q}\left\langle\left\langle\mathcal{E}_{q^{-1}(p \vee q)} \mid \mathcal{E}_{q^{-1}(p \vee q)}\right\rangle\right\rangle$ because $\left\langle\left\langle\mathcal{E}_{p} \mid \mathcal{E}_{p}\right\rangle\right\rangle\left\langle\left\langle\mathcal{E}_{q} \mid \mathcal{E}_{q}\right\rangle\right\rangle \subseteq\left\langle\left\langle\mathcal{E}_{p \vee q} \mid \mathcal{E}_{p \vee q}\right\rangle\right\rangle$ and $\mathcal{E}_{q}=\left\langle\left\langle\mathcal{E}_{q} \mid \mathcal{E}_{q}\right\rangle\right\rangle \mathcal{E}_{q}$ (see also Lemma 3.1.7). Similarly,

$$
\mathcal{E}_{r}^{*}\left\langle\left\langle\mathcal{E}_{s} \mid \mathcal{E}_{s}\right\rangle\right\rangle=\left(\left\langle\left\langle\mathcal{E}_{s} \mid \mathcal{E}_{s}\right\rangle\right\rangle \mathcal{E}_{r}\right)^{*} \subseteq\left(\mathcal{E}_{r}\left\langle\left\langle\mathcal{E}_{r^{-1}}(r \vee s) \mid \mathcal{E}_{r^{-1}(r \vee s)}\right\rangle\right)^{*}=\left\langle\left\langle\mathcal{E}_{r^{-1}(r \vee s)} \mid \mathcal{E}_{r^{-1}(r \vee s)}\right\rangle \mathcal{E}_{r}^{*}\right.\right.
$$

Applying again the fact that $\mathcal{E}$ is simplifiable to the ideals $\left\langle\left\langle\mathcal{E}_{q^{-1}(p \vee q)} \mid \mathcal{E}_{q^{-1}(p \vee q)}\right\rangle\right\rangle$ and $\left\langle\left\langle\mathcal{E}_{r^{-1}(r \vee s)} \mid \mathcal{E}_{r^{-1}(r \vee s)}\right\rangle\right\rangle$, we deduce that

$$
\mathcal{E}_{p}^{*} \otimes_{A} \mathcal{E}_{q} \otimes_{A} \mathcal{E}_{r}^{*} \otimes_{A} \mathcal{E}_{s} \hookrightarrow \mathcal{E}_{p}^{*} \otimes_{A} \mathcal{E}_{q} \cdot I \otimes_{A} I \cdot \mathcal{E}_{r}^{*} \otimes_{A} \mathcal{E}_{s}
$$


where $I=\left\langle\left\langle\mathcal{E}_{\left(r^{-1}(r \vee s)\right) \vee\left(q^{-1}(p \vee q)\right)} \mid \mathcal{E}_{\left(r^{-1}(r \vee s)\right) \vee\left(q^{-1}(p \vee q)\right)}\right\rangle\right\rangle$. By Theorem A.2.11 (see also 52, Proposition $3.24]), \mathcal{E}_{q} I=\left\langle\left\langle\mathcal{E}_{q} I \mid \mathcal{E}_{q} I\right\rangle\right\rangle \mathcal{E}_{q} I$ and $I \mathcal{E}_{r}^{*}=I \mathcal{E}_{r}^{*}\left\langle\left\langle\mathcal{E}_{r} I \mid \mathcal{E}_{r} I\right\rangle\right\rangle$. From this we obtain the inclusion

$$
\mathcal{E}_{p}^{*} \otimes_{A} \mathcal{E}_{q} \otimes_{A} \mathcal{E}_{r}^{*} \otimes_{A} \mathcal{E}_{s} \hookrightarrow \mathcal{E}_{p}^{*} \otimes_{A}\left\langle\left\langle\mathcal{E}_{p u} \mid \mathcal{E}_{p u}\right\rangle\right\rangle \otimes_{A} \mathcal{E}_{q} \otimes_{A} \mathcal{E}_{r}^{*} \otimes_{A}\left\langle\left\langle\mathcal{E}_{s v} \mid \mathcal{E}_{s v}\right\rangle\right\rangle_{A_{A}} \mathcal{E}_{s},
$$

because

$$
q\left(r^{-1}(r \vee s) \vee q^{-1}(p \vee q)\right)=q r^{-1}(r \vee s) \vee(p \vee q)=q r^{-1}(r \vee s) \vee p=p u
$$

and

$$
r\left(r^{-1}(r \vee s) \vee q^{-1}(p \vee q)\right)=(r \vee s) \vee r q^{-1}(p \vee q)=s \vee r q^{-1}(p \vee q)=s v
$$

Now the associativity of the multiplication on $\mathcal{E}$ implies that

$$
\left(\mu_{s, s^{-1}(r \vee s)}^{-1} \otimes 1\right) \mu_{r \vee s,(r \vee s)^{-1} s v}^{-1}=\left(1 \otimes \mu_{s^{-1}(r \vee s),(r \vee s)^{-1} s v}^{-1}\right) \mu_{s, v}^{-1}
$$

and

$$
\left(\mu_{r, r^{-1}(r \vee s)}^{-1} \otimes 1\right) \mu_{r \vee s,(r \vee s)^{-1} s v}^{-1}=\left(1 \otimes \mu_{r^{-1}(r \vee s),(r \vee s)^{-1} s v}^{-1}\right) \mu_{r, r^{-1} s v}^{-1} .
$$

These are two commutative diagrams:

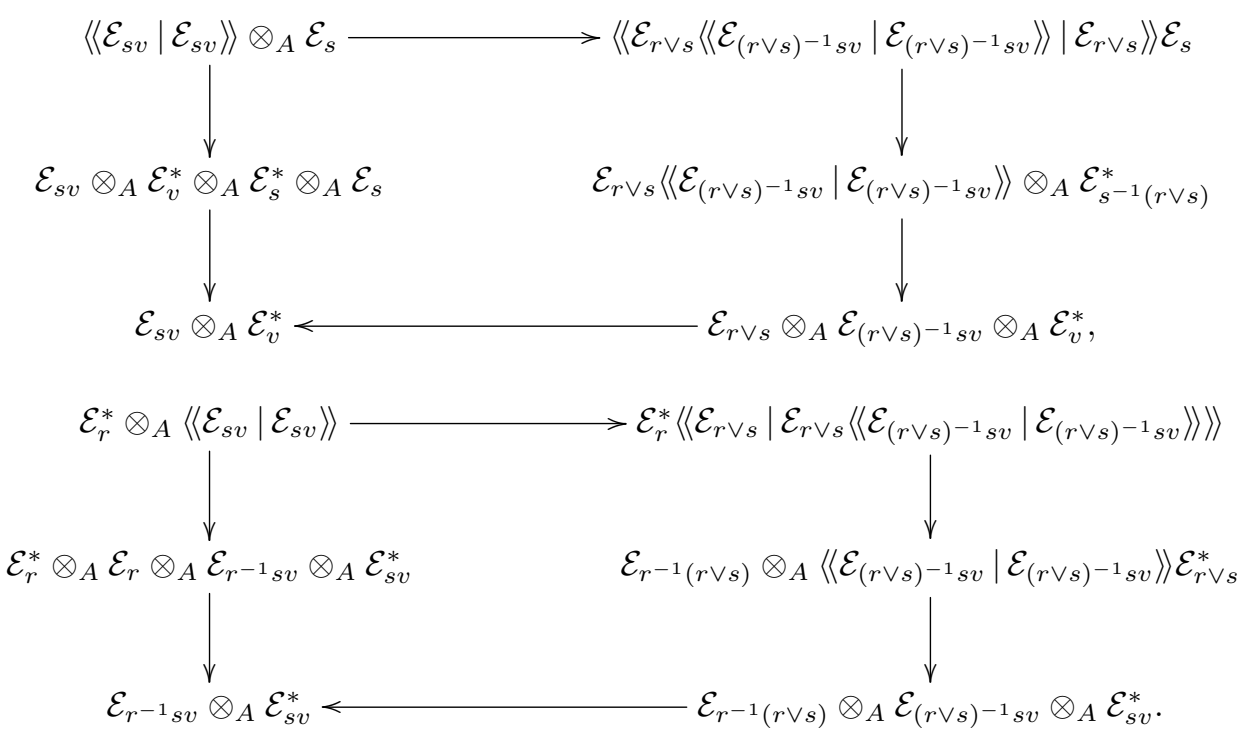

The same arguments show that the following diagrams commute:

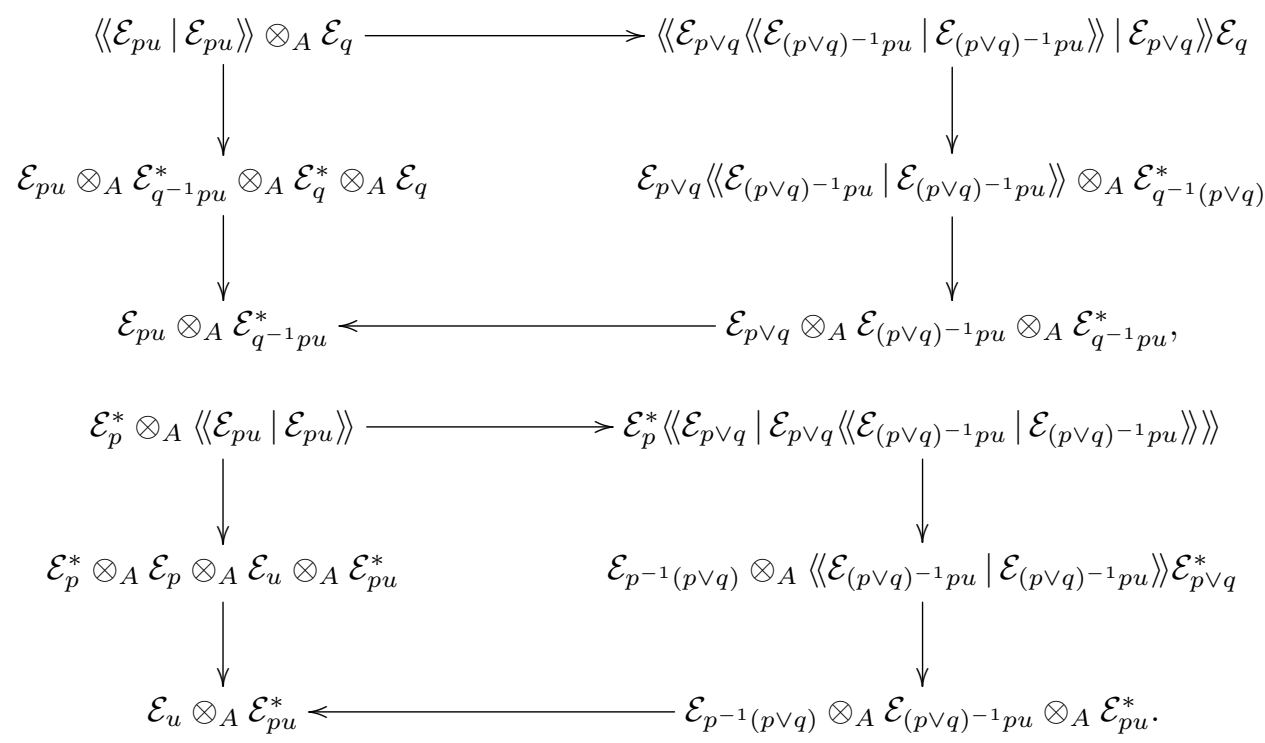




\section{FELL BUNDLES OVER QUASI-LATTICE ORDERED GROUPS}

Using again that the multiplication is associative on $\mathcal{E}$, we deduce the identity

$$
\mu_{r q^{-1}(p \vee q),(p \vee q)^{-1} p u}\left(\mu_{r, q^{-1}(p \vee q)} \otimes 1\right)=\mu_{r, q^{-1} p u}\left(1 \otimes \mu_{q^{-1}(p \vee q),(p \vee q)^{-1} p u}\right) .
$$

This produces the commutative diagram

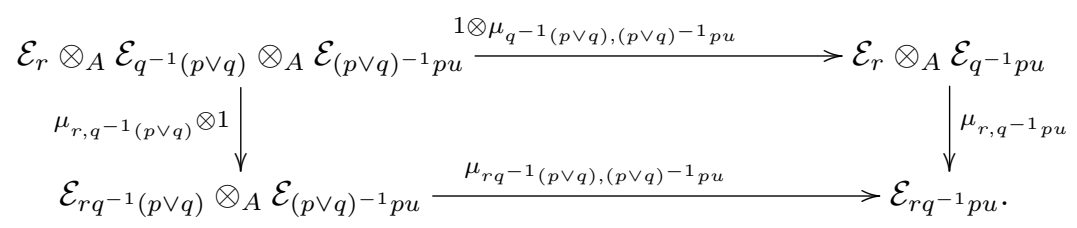

We have a similar commutative diagram for $q, r^{-1}(r \vee s)$ and $(r \vee s)^{-1} s v$. In addition, notice that $(p \vee q)^{-1} p u=\left(r q^{-1}(p \vee q)\right)^{-1} s v,(r \vee s)^{-1} s v=\left(q r^{-1}(r \vee s)\right)^{-1} p u$ and $q^{-1} p u=r^{-1} s v$. It follows from the above commutative diagrams that the following two inclusions coincide:

$$
\begin{aligned}
& \mathcal{E}_{p u}^{*} \otimes_{A} \mathcal{E}_{q} \otimes_{A} \mathcal{E}_{r}^{*} \otimes_{A} \mathcal{E}_{s v} \cong \mathcal{E}_{p u}^{*} \otimes_{A} \mathcal{E}_{q} \otimes_{A} \mathcal{E}_{r}^{*} \otimes_{A} \mathcal{E}_{r \vee s} \otimes_{A} \mathcal{E}_{(r \vee s)^{-1} s v} \\
& \cong \mathcal{E}_{p u}^{*} \otimes_{A} \mathcal{E}_{q} \otimes_{A}\left(\mathcal{E}_{r}^{*} \otimes_{A} \mathcal{E}_{r}\right) \otimes_{A} \mathcal{E}_{r^{-1}(r \vee s)} \otimes_{A} \mathcal{E}_{(r \vee s)^{-1} s v} \\
& \cong \mathcal{E}_{p u}^{*} \otimes_{A} \mathcal{E}_{q} \otimes_{A}\left\langle\mathcal{E}_{r} \mid \mathcal{E}_{r}\right\rangle \mathcal{E}_{r^{-1}(r \vee s)} \otimes_{A} \mathcal{E}_{(r \vee s)^{-1} s v} \\
& \hookrightarrow \mathcal{E}_{p u}^{*} \otimes_{A} \mathcal{E}_{q r^{-1}(r \vee s)} \otimes_{A} \mathcal{E}_{(r \vee s)^{-1} s v} \\
& \cong \mathcal{E}_{\left(q r^{-1}(r \vee s)\right)^{-1} p u}^{*} \otimes_{A} \mathcal{E}_{q r^{-1}(r \vee s)}^{*} \otimes_{A} \mathcal{E}_{q r^{-1}(r \vee s)} \otimes_{A} \mathcal{E}_{(r \vee s)^{-1} s v} \\
& \cong\left\langle\mathcal{E}_{\left(q r^{-1}(r \vee s)\right)^{-1} p u} \mid\left\langle\mathcal{E}_{q r^{-1}(r \vee s)} \mid \mathcal{E}_{q r^{-1}(r \vee s)}\right\rangle \mathcal{E}_{(r \vee s)^{-1} s v}\right\rangle \\
& =\left\langle\mathcal{E}_{\left(q r^{-1}(r \vee s)\right)^{-1} p u} \mid\left\langle\mathcal{E}_{r^{-1}(r \vee s)} \mid\left\langle\mathcal{E}_{q} \mid \mathcal{E}_{q}\right\rangle \mathcal{E}_{r^{-1}(r \vee s)}\right\rangle \mathcal{E}_{(r \vee s)^{-1} s v}\right\rangle \\
& \subseteq\left\langle\mathcal{E}_{q^{-1} p u} \mid \mathcal{E}_{r^{-1} s v}\right\rangle \text {; } \\
& \mathcal{E}_{p u}^{*} \otimes_{A} \mathcal{E}_{q} \otimes_{A} \mathcal{E}_{r}^{*} \otimes_{A} \mathcal{E}_{s v} \cong \mathcal{E}_{(p \vee q)^{-1} p u}^{*} \otimes_{A} \mathcal{E}_{p \vee q}^{*} \otimes_{A} \mathcal{E}_{q} \otimes_{A} \mathcal{E}_{r}^{*} \otimes_{A} \mathcal{E}_{s v} \\
& \cong \mathcal{E}_{(p \vee q)^{-1} p u}^{*} \otimes_{A} \mathcal{E}_{q^{-1}(p \vee q)}^{*} \otimes_{A}\left(\mathcal{E}_{q}^{*} \otimes_{A} \mathcal{E}_{q}\right) \otimes_{A} \mathcal{E}_{r}^{*} \otimes_{A} \mathcal{E}_{s v} \\
& \cong \mathcal{E}_{(p \vee q)^{-1} p u}^{*} \otimes_{A} \mathcal{E}_{q^{-1}(p \vee q)}^{*}\left\langle\mathcal{E}_{q} \mid \mathcal{E}_{q}\right\rangle \otimes_{A} \mathcal{E}_{r}^{*} \otimes_{A} \mathcal{E}_{s v} \\
& \hookrightarrow \mathcal{E}_{(p \vee q)^{-1} p u}^{*} \otimes_{A} \mathcal{E}_{r q^{-1}(p \vee q)}^{*} \otimes_{A} \mathcal{E}_{s v} \\
& \cong \mathcal{E}_{(p \vee q)^{-1} p u}^{*} \otimes_{A} \mathcal{E}_{r q^{-1}(p \vee q)}^{*} \otimes_{A} \mathcal{E}_{r q^{-1}(p \vee q)} \otimes_{A} \mathcal{E}_{\left(r q^{-1}(p \vee q)\right)^{-1} s v} \\
& \cong\left\langle\mathcal{E}_{(p \vee q)^{-1} p u} \mid\left\langle\mathcal{E}_{r q^{-1}(p \vee q)} \mid \mathcal{E}_{r q^{-1}(p \vee q)}\right\rangle \mathcal{E}_{\left(r q^{-1}(p \vee q)\right)^{-1} s v}\right\rangle \\
& =\left\langle\mathcal{E}_{(p \vee q)^{-1} p u} \mid\left\langle\mathcal{E}_{q^{-1}(p \vee q)} \mid\left\langle\mathcal{E}_{r} \mid \mathcal{E}_{r}\right\rangle \mathcal{E}_{q^{-1}(p \vee q)}\right\rangle \mathcal{E}_{\left(r q^{-1}(p \vee q)\right)^{-1} s v}\right\rangle \\
& \subseteq\left\langle\mathcal{E}_{q^{-1} p u} \mid \mathcal{E}_{r^{-1} s v}\right\rangle \text {. }
\end{aligned}
$$

Now let us go back to the diagram

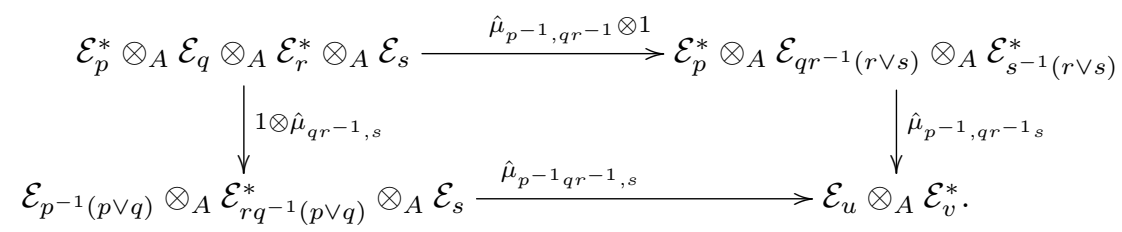

It follows from 5 that its top-right composite combines the top-right composites of 5 (5.1.11) and $(5.1 .12$ with $(5.1 .15)$ and the left arrow of (5.1.14). The left-bottom composite of $(5.1 .17)$ involves the top-right composites of $(5.1 .13)$ and $(5.1 .14)$, the inclusion $(5.1 .16)$ as well as the left arrows of (5.1.11). So applying the relations

$$
\begin{aligned}
& \mathcal{E}_{\left(q r^{-1}(r \vee s)\right)^{-1} p u}^{*}\left\langle\mathcal{E}_{r^{-1}(r \vee s)} \mid\left\langle\mathcal{E}_{q} \mid \mathcal{E}_{q}\right\rangle \mathcal{E}_{r^{-1}(r \vee s)}\right\rangle\left\langle\left\langle\mathcal{E}_{(r \vee s)^{-1} s v} \mid \mathcal{E}_{(r \vee s)^{-1} v}\right\rangle\right\rangle= \\
& \left\langle\mathcal{E}_{\left(q r^{-1}(r \vee s)\right)^{-1} p u} \mid\left\langle\mathcal{E}_{r^{-1}(r \vee s)} \mid\left\langle\mathcal{E}_{q} \mid \mathcal{E}_{q}\right\rangle \mathcal{E}_{r^{-1}(r \vee s)}\right\rangle \mathcal{E}_{(r \vee s)^{-1} s v}\right\rangle \mathcal{E}_{(r \vee s)^{-1} s v}^{*}
\end{aligned}
$$


and

$$
\begin{gathered}
\left\langle\left\langle\mathcal{E}_{(p \vee q)^{-1} p u} \mid \mathcal{E}_{(p \vee q)^{-1} p u}\right\rangle\right\rangle\left\langle\mathcal{E}_{q^{-1}(p \vee q)} \mid\left\langle\mathcal{E}_{r} \mid \mathcal{E}_{r}\right\rangle \mathcal{E}_{q^{-1}(p \vee q)}\right\rangle \mathcal{E}_{\left(r q^{-1}(p \vee q)\right)^{-1} s v}= \\
\mathcal{E}_{(p \vee q)^{-1} p u}\left\langle\mathcal{E}_{(p \vee q)^{-1} p u} \mid\left\langle\mathcal{E}_{q^{-1}(p \vee q)} \mid\left\langle\mathcal{E}_{r} \mid \mathcal{E}_{r}\right\rangle \mathcal{E}_{q^{-1}(p \vee q)}\right\rangle \mathcal{E}_{\left(r q^{-1}(p \vee q)\right)^{-1} s v}\right\rangle
\end{gathered}
$$

and using the equality of 5.1 .15 and $(5.1 .16)$, we deduce from the commutativity of the diagrams described above that the diagram (5.1.17) also commutes, as desired.

In order to prove the uniqueness property, let $\hat{\mathcal{E}}^{\prime}=\left(\hat{\mathcal{E}}_{g}^{\prime}\right)_{g \in G}$ be another Fell bundle that is semisaturated and orthogonal and extends the structure of product system of $\mathcal{E}$. Let $j^{\prime}=\left\{j_{p}^{\prime}\right\}_{p \in P}$ be the family of isomorphisms $\mathcal{E}_{p} \cong \hat{\mathcal{E}}_{p}^{\prime}$. We obtain an isomorphism of Hilbert bimodules $j_{p^{-1}}^{\prime}: \mathcal{E}_{p}^{*} \rightarrow \hat{\mathcal{E}}_{p^{-1}}^{\prime}$ through the composite

$$
\mathcal{E}_{p}^{*} \stackrel{*}{\rightarrow} \mathcal{E}_{p} \stackrel{j_{p}^{\prime}}{\rightarrow} \hat{\mathcal{E}}_{p}^{\prime} \stackrel{*}{\rightarrow} \hat{\mathcal{E}}_{p^{-1}}^{\prime},
$$

where the arrow involved in the left-hand side is the canonical conjugate-linear map and "** in the right-hand side is the involution in $\left(\hat{\mathcal{E}}_{g}^{\prime}\right)_{g \in G}$. This is indeed an isomorphism of complex vector spaces since the involution operation on $\hat{\mathcal{E}}^{\prime}$ is also a conjugate-linear map. Furthermore, the ideal in $A$ determined by $j_{p}^{\prime}\left(\mathcal{E}_{p}\right) j_{p}^{\prime}\left(\mathcal{E}_{p}\right)^{*}$ is contained in $j_{e}^{\prime}\left(\left\langle\left\langle\mathcal{E}_{p} \mid \mathcal{E}_{p}\right\rangle\right\rangle\right)$ because $\mathcal{E}_{p}=\left\langle\left\langle\mathcal{E}_{p} \mid \mathcal{E}_{p}\right\rangle\right\rangle \mathcal{E}_{p}$ and $j^{\prime}$ preserves the multiplication on $\mathcal{E}$. And for all $\xi, \eta$ and $\zeta \in \mathcal{E}_{p}$, we have that

$$
j_{e}^{\prime}(\langle\langle\xi \mid \eta\rangle\rangle) j_{p}^{\prime}(\zeta)=j_{p}^{\prime}(\xi\langle\eta \mid \zeta\rangle)=j_{p}^{\prime}(\xi) j_{e}^{\prime}(\langle\eta \mid \zeta\rangle)=j_{p}^{\prime}(\xi) j_{p}^{\prime}(\eta)^{*} j_{p}^{\prime}(\zeta) .
$$

This implies that $j_{e}^{\prime}(\langle\langle\xi \mid \eta\rangle\rangle)=j_{p}^{\prime}(\xi) j_{p}^{\prime}(\eta)^{*}=j_{p^{-1}}^{\prime}\left(\xi^{*}\right)^{*} j_{p^{-1}}^{\prime}\left(\eta^{*}\right)$ because $\left\langle\left\langle\mathcal{E}_{p} \mid \mathcal{E}_{p}\right\rangle\right\rangle$ acts faithfully on $\mathcal{E}_{p}$ and $j_{e}^{\prime}$ is also injective. So we let $j_{g}^{\prime}$ be the zero map if $g \vee e=\infty$ and for $g \in G$ with $g \vee e<\infty$, we let $j_{g}^{\prime}: \mathcal{E}_{g \vee e} \otimes_{A} \mathcal{E}_{g^{-1} \vee e}^{*} \rightarrow \hat{\mathcal{E}}_{g}^{\prime}$ be defined on elementary tensors by $\xi \otimes \eta^{*} \mapsto j_{g \vee e}^{\prime}(\xi) j_{\left(g^{-1} \vee e\right)^{-1}}^{\prime}\left(\eta^{*}\right)$. This is isometric because $j_{g \vee e}^{\prime}$ and $j_{\left(g^{-1} \vee e\right)^{-1}}^{\prime}$ preserve inner products. These maps are surjective because $\hat{\mathcal{E}}^{\prime}$ is semi-saturated and orthogonal. Since it extends $\mathcal{E}$ and $j_{p^{-1}}^{\prime}$ preserves the structure of Hilbert bimodule of $\mathcal{E}_{p}^{*}$, it follows that $\left\{j_{g}^{\prime}\right\}_{g \in G}$ is an isomorphism between Fell bundles. This completes the proof of the theorem.

Corollary 5.1.18. Let $\mathcal{E}=\left(\mathcal{E}_{p}\right)_{p \in P}$ be a simplifiable product system of Hilbert bimodules. For each $p \in P$, set $I_{p}:=\left\langle\left\langle\mathcal{E}_{p} \mid \mathcal{E}_{p}\right\rangle\right\rangle$ and $\mathcal{I}=\left\{I_{p}\right\}_{p \in P}$. Then the canonical ${ }^{*}$-homomorphism from $A$ to the relative Cuntz-Pimsner algebra $\mathcal{O}_{\mathcal{I}, \mathcal{E}}$ is an isomorphism onto the gauge-fixed point algebra $\mathcal{O}_{\mathcal{I}, \mathcal{E}}^{e}$. Moreover, $\mathcal{O}_{\mathcal{I}, \mathcal{E}}^{p} \cong \mathcal{E}_{p}$ for all $p \in P$.

Proof. Let $\left(\hat{\mathcal{E}}_{g}\right)_{g \in G}$ be the Fell bundle associated to $\mathcal{E}$ as in the previous theorem and let $\mathrm{C}^{*}\left(\left(\hat{\mathcal{E}}_{g}\right)_{g \in G}\right)$ be its full cross sectional $\mathrm{C}^{*}$-algebra. Let $\hat{j}=\left\{\hat{j}_{g}\right\}_{g \in G}$ denote the canonical representation of $\left(\hat{\mathcal{E}}_{g}\right)_{g \in G}$ in $\mathrm{C}^{*}\left(\left(\hat{\mathcal{E}}_{g}\right)_{g \in G}\right)$. Since $\left(\hat{\mathcal{E}}_{g}\right)_{g \in G}$ extends the structure of $\mathcal{E}$, there is a canonical representation of $\mathcal{E}$ in $\mathrm{C}^{*}\left(\left(\hat{\mathcal{E}}_{g}\right)_{g \in G}\right)$ obtained by

$$
\mathcal{E}_{p} \ni \xi \mapsto \hat{j}_{p}(\xi)
$$

Given $p \in P$ and $\xi, \eta \in \mathcal{E}_{p}$, the multiplication $\xi \cdot \eta^{*}$ in $\left(\hat{\mathcal{E}}_{g}\right)_{g \in G}$ is precisely the left inner product $\langle\langle\xi \mid \eta\rangle\rangle$, so that the representation of $\mathcal{E}$ in $\mathrm{C}^{*}\left(\left(\hat{\mathcal{E}}_{g}\right)_{g \in G}\right)$ is Cuntz-Pimsner covariant on $\mathcal{I}$. As a result, the canonical map from $A$ to $\mathcal{O}_{\mathcal{I}, \mathcal{E}}$ is injective. Since $\mathcal{E}$ is simplifiable, its representation in $\mathcal{O}_{\mathcal{I}, \mathcal{E}}$ is Nica covariant by Proposition 5.1.7. Thus, $\mathcal{O}_{\mathcal{I}, \mathcal{E}}^{e}$ is the closed linear span of the set

$$
\left\{j_{p}(\xi) j_{p}(\eta)^{*} \mid \xi, \eta \in \mathcal{E}_{p}, p \in P\right\}
$$

So the Cuntz-Pimsner covariance condition implies that $j_{e}: A \rightarrow \mathcal{O}_{\mathcal{I}, \mathcal{E}}^{e}$ is an isomorphism.

It follows that $j_{p}: \mathcal{E}_{p} \rightarrow \mathcal{O}_{\mathcal{I}, \mathcal{E}}^{p}$ is injective for all $p \in P$. Again because the representation of $\mathcal{E}$ in $\mathcal{O}_{\mathcal{I}, \mathcal{E}}$ is Nica covariant, $\mathcal{O}_{\mathcal{I}, \mathcal{E}}^{p}$ is generated by elements of the form $j_{q}(\xi) j_{r}(\eta)^{*}$ with $q r^{-1}=p$. Using that $\mu_{p, r}$ is a correspondence isomorphism, we deduce from Cuntz-Pimsner covariance that $j_{p}$ is also surjective, as asserted.

Remark 5.1.19. In order to build a Fell bundle out of a simplifiable product system in the proof of Theorem 5.1.8, we defined the fibres as well as the operations of a Fell bundle explicitly and established the required axioms for Fell bundles, such as associativity of the multiplication maps. From this we derived Corollary 5.1.18. One could also prove Corollary 5.1.18 by using Theorem 6.2.5 


\section{FELL BUNDLES OVER QUASI-LATTICE ORDERED GROUPS}

and Proposition 6.3.8 Thus Theorem 5.1.8 would follow as a consequence. In order to obtain a self-contained theory, we have chosen to construct the Fell bundle out of a simplifiable product system of Hilbert bimodules explicitly. We will build an equivalence between the corresponding bicategories in a similar fashion.

\subsection{The equivalence of bicategories}

Let $\left(B_{g}\right)_{g \in G}$ be a semi-saturated Fell bundle with respect to $(G, P)$. There is a canonical product system associated to $\left(B_{g}\right)_{g \in G}$. Indeed, for each $p \in P$, view $B_{p}$ as a Hilbert $B_{e}$-bimodule with left and right actions inherited from the multiplication in $\left(B_{g}\right)_{g \in G}$. The left inner product is given by $\langle\langle\xi \mid \eta\rangle\rangle:=\xi \eta^{*}$, while the right inner product is $\langle\xi \mid \eta\rangle:=\xi^{*} \eta$. The property (S1) of Definition 5.1.1 says that $\mathcal{B}=\left(B_{p}\right)_{p \in P}$ is a product system with isomorphisms $B_{p} \otimes_{B_{e}} B_{q} \cong B_{p q}$ coming from the multiplication in $\left(B_{g}\right)_{g \in G}$. If $\left(B_{g}\right)_{g \in G}$ is also orthogonal, the next result states that the cross sectional $\mathrm{C}^{*}$-algebra of $\left(B_{g}\right)_{g \in G}$ can be recovered from $\mathcal{B}$.

Proposition 5.2.1. Let $\left(B_{g}\right)_{g \in G}$ be a Fell bundle that is semi-saturated and orthogonal with respect to $(G, P)$. Then $\mathcal{B}=\left(B_{p}\right)_{p \in P}$ is a simplifiable product system of Hilbert bimodules. Its relative Cuntz-Pimsner algebra $\mathcal{O}_{\mathcal{I}, \mathcal{B}}$ is naturally isomorphic to the cross sectional $\mathrm{C}^{*}$-algebra of $\left(B_{g}\right)_{g \in G}$.

Proof. Let $p, q \in P$ and set $g=p^{-1} q$. Notice that $p \vee q=\infty$ if and only if $g \vee e=\infty$ and hence $\left\langle\left\langle B_{p} \mid B_{p}\right\rangle\right\rangle\left\langle\left\langle B_{q} \mid B_{q}\right\rangle\right\rangle=B_{p} B_{p}^{*} B_{q} B_{q}^{*}=\{0\}$ provided $p \vee q=\infty$. Suppose that $p \vee q<\infty$. Then $g \vee e=p^{-1}(p \vee q)$ and $g^{-1} \vee e=q^{-1}(p \vee q)$ so that

$$
\begin{aligned}
\left\langle\left\langle B_{p} \mid B_{p}\right\rangle\right\rangle\left\langle\left\langle B_{q} \mid B_{q}\right\rangle\right\rangle & \subseteq B_{p} B_{p^{-1} q} B_{q}^{*} \\
& =B_{p} B_{p^{-1}(p \vee q)} B_{q^{-1}(p \vee q)}^{*} B_{q}^{*} \\
& =B_{p \vee q} B_{p \vee q}^{*}=\left\langle\left\langle B_{p \vee q} \mid B_{p \vee q}\right\rangle\right\rangle .
\end{aligned}
$$

The representation of $\left(B_{g}\right)_{g \in G}$ in $\mathrm{C}^{*}\left(\left(B_{g}\right)_{g \in G}\right)$ restricted to the fibres over $P$ is Cuntz-Pimsner covariant on $\mathcal{I}$. This gives us a ${ }^{*}$-homomorphism $\psi: \mathcal{O}_{\mathcal{I}, \mathcal{B}} \rightarrow \mathrm{C}^{*}\left(\left(B_{g}\right)_{g \in G}\right)$. In order to build the inverse of $\psi$, let us define a representation $\phi=\left\{\phi_{g}\right\}_{g \in G}$ of $\left(B_{g}\right)_{g \in G}$ in $\mathcal{O}_{\mathcal{I}, \mathcal{B}}$.

Let $g \in G$. If $g \vee e=\infty$, then $\mathcal{O}_{\mathcal{B}, \mathcal{I}}^{g}=\{0\}=B_{g}$ and $\phi_{g}$ is the zero map. For $g=p \in P$, we set $\phi_{p}:=j_{p}$. Now let $g \in G$ with $g \vee e<\infty$ and let $\xi \in B_{g}$ be of the form $\eta \zeta^{*}$ with $\eta \in B_{g \vee e}$ and $\zeta \in B_{g^{-1} \vee e}$. Set $\phi_{g}(\xi):=j_{g \vee e}(\eta) j_{g^{-1} \vee e}(\zeta)^{*}$. Given $\eta, \eta^{\prime} \in B_{g \vee e}$ and $\zeta, \zeta^{\prime} \in B_{g^{-1} \vee e}$, the CuntzPimsner covariance condition entails

$$
\left.j_{g^{-1} \vee e}(\zeta) j_{g \vee e}(\eta)^{*} j_{g \vee e}\left(\eta^{\prime}\right) j_{g^{-1} \vee e}\left(\zeta^{\prime}\right)^{*}=j_{e}\left(\left\langle\zeta \zeta \mid\left\langle\eta \mid \eta^{\prime}\right\rangle \zeta^{\prime}\right\rangle\right\rangle\right)=\phi_{e}\left(\left(\eta \zeta^{*}\right)^{*}\left(\eta^{\prime} \zeta^{\prime *}\right)\right)
$$

Hence the axiom $\|b\|^{2}=\left\|b^{*} b\right\|$ applied to $b \in B_{g}$ ensures that $\phi_{g}$ extends to a continuous linear map from $B_{g}=B_{g \vee e} B_{g^{-1} \vee e}$ to $\mathcal{O}_{\mathcal{I}, \mathcal{B}}$, which we still denote by $\phi_{g}$. By definition, $\phi_{g}(\xi)^{*}=\phi_{g^{-1}}\left(\xi^{*}\right)$ for all $\xi \in B_{g}$ and $g \in G$. So it remains to prove that $\phi_{g h}(\xi \eta)=\phi_{g}(\xi) \phi_{h}(\eta)$ for all $\xi \in B_{g}, \eta \in B_{h}$ and $g, h \in G$. This clearly holds if $g, h \in P$ because the representation of $\mathcal{B}$ in $\mathcal{O}_{\mathcal{I}, \mathcal{B}}$ preserves multiplication. In addition, since the representation of $\mathcal{B}$ in $\mathcal{O}_{\mathcal{I}, \mathcal{B}}$ is Nica covariant, given $p, q \in P$, $\xi \in B_{p}$ and $\eta \in B_{q}$, it follows that $j_{p}(\xi)^{*} j_{q}(\eta)=0$ whenever $p \vee q=\infty$. In case $p \vee q<\infty$, it follows that

$$
B_{p}^{*} B_{q} \subseteq B_{p}^{*} B_{p \vee q} B_{p \vee q}^{*} B_{q} .
$$

We deduce from the Cuntz-Pimsner covariance condition that, for all $\zeta, \zeta^{\prime} \in \mathcal{E}_{p \vee q}$,

$$
\left.j_{p}(\xi)^{*} j_{e}\left(\left\langle\zeta \zeta \mid \zeta^{\prime}\right\rangle\right\rangle\right) j_{q}(\eta)=j_{p}(\xi)^{*} j_{p \vee q}(\zeta) j_{p \vee q}\left(\zeta^{\prime}\right)^{*} j_{q}(\eta)
$$

Combining this with the fact that $j=\left\{j_{p}\right\}_{p \in P}$ is a representation of $\mathcal{B}$, we conclude that $\phi_{g h}(\xi \eta)=$ $\phi_{g}(\xi) \phi_{h}(\eta)$ for all $\xi \in B_{g}, \eta \in B_{h}$ and $g, h \in G$. Therefore, this induces a *-homomorphism $\hat{\phi}: \mathrm{C}^{*}\left(\left(B_{g}\right)_{g \in G}\right) \rightarrow \mathcal{O}_{\mathcal{I}, \mathcal{B}}$, which is the inverse of $\psi$.

Combining Example 5.1.4 with the previous proposition, we obtain the following:

Corollary 5.2.2. Let $\mathcal{E}=\left(\mathcal{E}_{p}\right)_{p \in P}$ be a compactly aligned product system and $\mathcal{O}_{\mathcal{J}, \mathcal{E}}$ a relative CuntzPimsner algebra associated to $\mathcal{E}$. Then $\left(\mathcal{O}_{\mathcal{J}, \mathcal{E}}^{p}\right)_{p \in P}$ is simplifiable. 
Definition 5.2.3. Let $(G, P)$ be a quasi-lattice order. A Fell bundle over $G$ is said to be extended from $P$ if it is semi-saturated and orthogonal with respect to the quasi-lattice ordered group structure of $(G, P)$.

Definition 5.2.4. Let $\left(B_{g}\right)_{g \in G}$ and $\left(C_{g}\right)_{g \in G}$ be Fell bundles extended from $P$. A correspondence $(\mathcal{F}, U):\left(B_{g}\right)_{g \in G} \rightarrow\left(C_{g}\right)_{g \in G}$ consists of a $\mathrm{C}^{*}$-correspondence $\mathcal{F}: B_{e} \leadsto C_{e}$ and a family of isometries $U=\left\{U_{g}\right\}_{g \in G}$, where $U_{g}: B_{g} \otimes_{B_{e}} \mathcal{F} \rightarrow \mathcal{F} \otimes_{C_{e}} C_{g}$, such that $U_{e}: B_{e} \otimes_{B_{e}} \mathcal{F} \cong \mathcal{F} \otimes_{C_{e}} C_{e}$ is the isomorphism which sends $b \otimes(\xi c)$ to $\psi(b) \xi \otimes c$ and, for all $p \in P, U_{p}$ is unitary. Here we are regarding the $B_{g}$ 's as correspondences over $B_{e}$. We also require the following diagram to commute for all $g, h \in G$ :

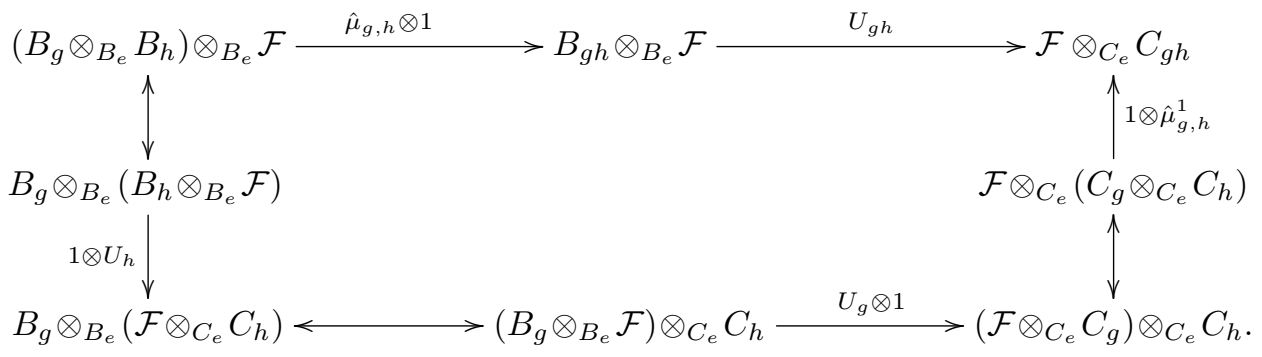

A correspondence $(\mathcal{F}, U)$ is proper if $\mathcal{F}$ is a proper correspondence.

It is unclear to us whether or not all of the $U_{g}$ 's in the above definition are unitary whenever the $U_{p}$ 's are so.

Definition 5.2.6. We will denote by $\mathfrak{C}^{(G, P)}$ the bicategory whose objects are Fell bundles over $G$ extended from $P$ and arrows $\left(B_{g}\right)_{g \in G} \rightarrow\left(C_{g}\right)_{g \in G}$ are correspondences as above. A 2-morphism $w:\left(\mathcal{F}_{0}, U_{0}\right) \Rightarrow\left(\mathcal{F}_{1}, U_{1}\right)$ is a correspondence isomorphism $w: \mathcal{F}_{0} \rightarrow \mathcal{F}_{1}$ making the following diagram commute for all $g \in G$ :

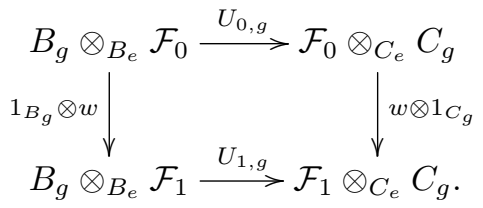

The unit arrow on an object $\left(B_{g}\right)_{g \in G}$ is the identity correspondence $B_{e}: B_{e} \rightarrow B_{e}$ with the family of isomorphisms $\hat{\iota}_{G}=\left\{\iota_{B_{g}}\right\}_{g \in G}$, where $\iota_{B_{g}}$ is the isomorphism $B_{e} \otimes_{B_{e}} B_{g} \cong B_{g} \otimes_{B_{e}} B_{e}$ obtained as in Definition 4.3.3. The further data needed for a bicategory is also defined as in Definition 4.3.3 We let $\mathfrak{C}_{\mathrm{pr}}^{(G, P)}$ be the sub-bicategory of $\mathfrak{C}^{(G, P)}$ whose arrows are proper correspondences.

Lemma 5.2.7. Let $(\mathcal{F}, U):\left(B_{g}\right)_{g \in G} \rightarrow\left(C_{g}\right)_{g \in G}$ be a morphism in $\mathfrak{C}^{(G, P)}$. Then its restriction to the positive fibres is a covariant correspondence

$$
\left(B_{e}, \mathcal{B}, \mathcal{I}_{\mathcal{B}}\right) \rightarrow\left(C_{e}, \mathcal{C}, \mathcal{I}_{\mathcal{C}}\right)
$$

where $\mathcal{I}_{\mathcal{B}}$ and $\mathcal{I}_{\mathcal{C}}$ denote the families of Katsura's ideals for $\mathcal{B}$ and $\mathcal{C}$, respectively.

Proof. Let $(\mathcal{F}, U)$ be a correspondence from $\left(B_{g}\right)_{g \in G}$ to $\left(C_{g}\right)_{g \in G}$. By definition, $U_{p}: B_{p} \otimes_{B_{e}} \mathcal{F} \rightarrow$ $\mathcal{F} \otimes{ }_{C_{e}} C_{p}$ is unitary whenever $p$ belongs to the positive cone $P$. Thus, all we need to prove is that the ideal $\left\langle\left\langle B_{p} \mid B_{p}\right\rangle\right\rangle$ maps $\mathcal{F}$ into $\mathcal{F}\left\langle\left\langle C_{p} \mid C_{p}\right\rangle\right\rangle$. This follows from 5.2 .5 . We let $p^{-1}$ play the role of $q$ and obtain the commutative diagram

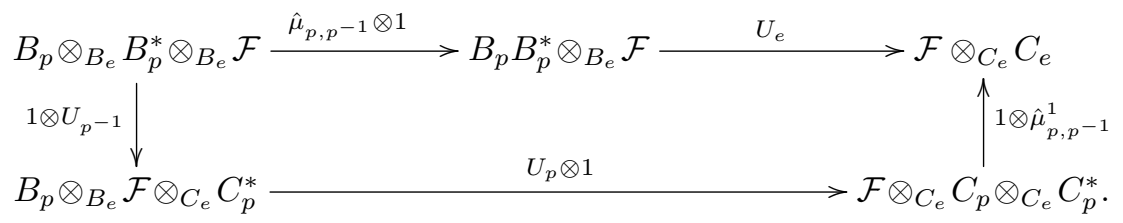

The image of the top map is $\left\langle\left\langle B_{p} \mid B_{p}\right\rangle\right\rangle \mathcal{F}$ and the image of the right map is $\mathcal{F}\left\langle\left\langle C_{p} \mid C_{p}\right\rangle\right\rangle$. Hence $\left\langle\left\langle B_{p} \mid B_{p}\right\rangle\right\rangle \mathcal{F} \subseteq \mathcal{F}\left\langle\left\langle C_{p} \mid C_{p}\right\rangle\right\rangle$. 


\section{FELL BUNDLES OVER QUASI-LATTICE ORDERED GROUPS}

Lemma 5.2.8. Let $\mathcal{E}=\left(\mathcal{E}_{p}\right)_{p \in P}$ and $\mathcal{G}=\left(\mathcal{G}_{p}\right)_{p \in P}$ be simplifiable product systems of Hilbert bimodules and let $\left(\hat{\mathcal{E}}_{g}\right)_{g \in G}$ and $\left(\hat{\mathcal{G}}_{g}\right)_{g \in G}$ be the associated Fell bundles extended from P. Let $(\mathcal{F}, V):\left(A, \mathcal{E}, \mathcal{I}_{\mathcal{E}}\right) \rightarrow$ $\left(B, \mathcal{G}, \mathcal{I}_{\mathcal{G}}\right)$ be a covariant correspondence. There is a correspondence $\left(\mathcal{F}^{\sharp}, U\right):\left(\hat{\mathcal{E}}_{g}\right)_{g \in G} \rightarrow\left(\hat{\mathcal{G}}_{g}\right)_{g \in G}$ such that $\mathcal{F}^{\sharp}=\mathcal{F}$ as a $\mathrm{C}^{*}$-correspondence and $U_{p}=V_{p}$ for all $p \in P$. Moreover, if $U^{\prime}=\left\{U_{g}^{\prime}\right\}_{g \in G}$ is another family of isometries turning $\mathcal{F}$ into a correspondence from $\left(\hat{\mathcal{E}}_{g}\right)_{g \in G}$ to $\left(\hat{\mathcal{G}}_{g}\right)_{g \in G}$ and such that $U_{p}^{\prime}=V_{p}$ for all $p \in P$, then $U_{g}^{\prime}=U_{g}$ for all $g \in G$.

Proof. We begin by defining the family of isometries $U=\left\{U_{g}\right\}_{g \in G}$. For $g \in G$ satisfying $g \vee e=\infty$, we let $U_{g}$ be the zero map. If $p \in P$, we put $U_{p}=V_{p}$. Using the inclusion $\left\langle\left\langle\mathcal{E}_{p} \mid \mathcal{E}_{p}\right\rangle\right\rangle \mathcal{F} \subseteq \mathcal{F}\left\langle\left\langle\mathcal{G}_{p} \mid \mathcal{G}_{p}\right\rangle\right\rangle$, we obtain an isometry $U_{p^{-1}}: \mathcal{E}_{p}^{*} \otimes_{A} \mathcal{F} \hookrightarrow \mathcal{F} \otimes_{B} \mathcal{G}_{p}^{*}$ for each $p \in P$ through the embedding

$$
\begin{array}{rlr}
\mathcal{E}_{p}^{*} \otimes_{A} \mathcal{F} & \cong \mathcal{E}_{p}^{*} \otimes_{A}\left\langle\left\langle\mathcal{E}_{p} \mid \mathcal{E}_{p}\right\rangle\right\rangle \otimes_{A} \mathcal{F} & \left(1_{\mathcal{E}_{p}^{*}} \otimes V_{e}\right) \\
& \hookrightarrow \mathcal{E}_{p}^{*} \otimes_{A} \mathcal{F} \otimes_{B}\left\langle\left\langle\mathcal{G}_{p} \mid \mathcal{G}_{p}\right\rangle\right\rangle & \left(1_{\mathcal{E}_{p}^{*}} \otimes U_{p}^{-1} \otimes 1_{\mathcal{G}_{p}^{*}}\right) \\
& \cong \mathcal{E}_{p}^{*} \otimes_{A} \mathcal{F} \otimes_{B} \mathcal{G}_{p} \otimes_{B} \mathcal{G}_{p}^{*} \\
& \cong \mathcal{E}_{p}^{*} \otimes_{A} \mathcal{E}_{p} \otimes_{A} \mathcal{F} \otimes_{B} \otimes_{B} \mathcal{G}_{p}^{*} \\
& \cong\left\langle\mathcal{E}_{p} \mid \mathcal{E}_{p}\right\rangle \otimes_{A} \mathcal{F} \otimes_{B} \mathcal{G}_{p}^{*} \\
& \hookrightarrow \mathcal{F} \otimes_{B} \mathcal{G}_{p}^{*}
\end{array}
$$

Finally, given $g \in G \backslash\left(P \cup P^{-1}\right)$ with $g \vee e<\infty$, we let $U_{g}$ be the composite

$$
\mathcal{E}_{g \vee e} \otimes_{A} \mathcal{E}_{g^{-1} \vee e}^{*} \otimes_{A} \mathcal{F} \stackrel{1 \otimes U_{\left(g^{-1} \vee e\right)^{-1}}}{\longrightarrow} \mathcal{E}_{g \vee e} \otimes_{A} \mathcal{F} \otimes_{B} \mathcal{G}_{g^{-1} \vee e} \stackrel{V_{g \vee e} \otimes 1}{\cong} \mathcal{F} \otimes_{B} \mathcal{G}_{g \vee e} \otimes_{B} \mathcal{G}_{g^{-1} \vee e}^{*}
$$

We set $U=\left\{U_{g}\right\}_{g \in G}$ and $\mathcal{F}^{\sharp}:=\mathcal{F}$. In order to prove that $\left(\mathcal{F}^{\sharp}, U\right)$ is a correspondence $\left(\hat{\mathcal{E}}_{g}\right)_{g \in G} \rightarrow$ $\left(\hat{\mathcal{G}}_{g}\right)_{g \in G}$, let us first establish the commutativity of the diagram

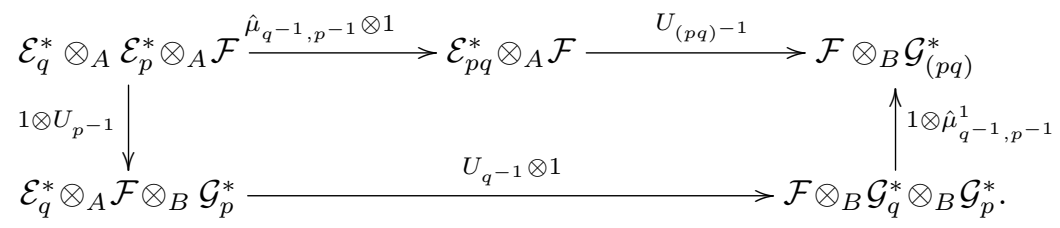

Observe that

$$
\begin{aligned}
\mathcal{E}_{q}^{*} \otimes_{A} \mathcal{E}_{p}^{*} \otimes_{A} \mathcal{F} & \cong \mathcal{E}_{q}^{*} \otimes_{A} \mathcal{E}_{p}^{*} \otimes_{A}\left\langle\left\langle\mathcal{E}_{p q} \mid \mathcal{E}_{p q}\right\rangle\right\rangle \otimes_{A} \mathcal{F} \\
& \hookrightarrow \mathcal{E}_{q}^{*} \otimes_{A} \mathcal{E}_{p}^{*} \otimes_{A} \mathcal{F} \otimes_{B}\left\langle\left\langle\mathcal{G}_{p q} \mid \mathcal{G}_{p q}\right\rangle\right\rangle \\
& \cong \mathcal{E}_{q}^{*} \otimes_{A} \mathcal{E}_{p}^{*} \otimes_{A} \mathcal{F} \otimes_{B}\left\langle\left\langle\mathcal{G}_{p}\left\langle\left\langle\mathcal{G}_{q} \mid \mathcal{G}_{q}\right\rangle\right\rangle \mid \mathcal{G}_{p}\right\rangle\right\rangle .
\end{aligned}
$$

So using that $V_{p}$ and $V_{q}$ intertwine the actions of $A$ and $B$ and the coherence axiom 4.3.2), we see that the above diagram commutes. Now since we have proven that the pair $\left(\mathcal{F}^{\sharp}, U\right)$ makes $(5.2 .5)$ commute if $g, h \in P^{-1}$ and the same is true for $g, h$ in $P$, it suffices to show that 5.2 .5 is commutative for $g \in P^{-1}$ and $h \in P$ with $g^{-1} \vee h<\infty$. This corresponds to the diagram

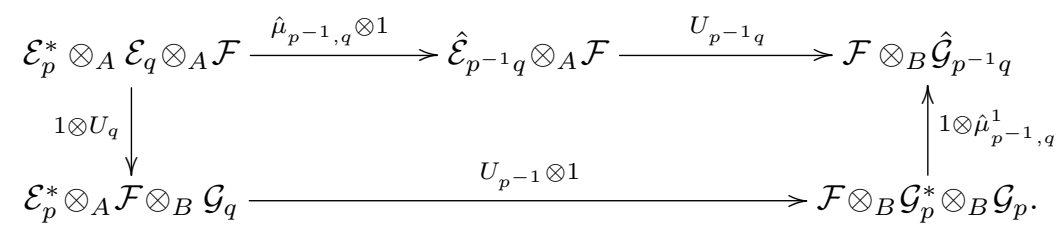

As a first step, we claim that 5.2.9 commutes when one replaces either $p$ or $q$ by $p \vee q$. Indeed, notice that

$$
\mathcal{E}_{p \vee q}^{*} \otimes_{A} \mathcal{E}_{q} \otimes_{A} \mathcal{F}=\mathcal{E}_{p \vee q}^{*} \otimes_{A} \mathcal{E}_{q} \otimes_{A} \mathcal{F}\left\langle\left\langle\mathcal{G}_{q^{-1}(p \vee q)} \mid \mathcal{G}_{q^{-1}(p \vee q)}\right\rangle\right\rangle\left\langle\mathcal{G}_{q} \mid \mathcal{G}_{q}\right\rangle
$$

because $\left\langle\left\langle\mathcal{E}_{p \vee q} \mid \mathcal{E}_{p \vee q}\right\rangle\right\rangle \operatorname{maps} \mathcal{E}_{q}$ into $\left\langle\left\langle\mathcal{E}_{q^{-1}(p \vee q)} \mid \mathcal{E}_{q^{-1}(p \vee q)}\right\rangle\right\rangle$ and $\mathcal{E}_{q} \otimes_{A} \mathcal{F} \cong \mathcal{F} \otimes_{B} \mathcal{G}_{q}$. Then an elementary tensor of $\mathcal{E}_{p \vee q}^{*} \otimes_{A} \mathcal{E}_{q} \otimes_{A} \mathcal{F}$ may be written as $\eta^{*} \otimes \xi \otimes_{A} \zeta b$, where $\eta \in \mathcal{E}_{p \vee q}, \xi \in \mathcal{E}_{q}, \zeta \in \mathcal{F}$ and $b$ lies in the ideal $\left\langle\left\langle\mathcal{G}_{q^{-1}(p \vee q)} \mid \mathcal{G}_{q^{-1}(p \vee q)}\right\rangle\left\langle\left\langle\mathcal{G}_{q} \mid \mathcal{G}_{q}\right\rangle\right.\right.$. In addition, for all $\xi_{1}, \xi_{2}, \zeta$ in $\mathcal{G}_{q}$ and $\eta_{1}, \eta_{2}$ in $\mathcal{G}_{q^{-1}(p \vee q)}$, we 
have that

$$
\begin{aligned}
\xi_{1}\left\langle\left\langle\eta_{1} \mid \eta_{2}\right\rangle\right\rangle\left\langle\xi_{2} \mid \zeta\right\rangle & =\left\langle\left\langle\xi_{1}\left\langle\left\langle\eta_{1} \mid \eta_{2}\right\rangle\right\rangle \mid \xi_{2}\right\rangle\right\rangle \zeta \\
& =\left\langle\left\langle\mu_{q, q^{-1}(p \vee q)}^{1}\left(\xi_{1} \otimes \eta_{1}\right) \mid \mu_{q, q^{-1}(p \vee q)}^{1}\left(\xi_{2} \otimes \eta_{2}\right)\right\rangle\right\rangle \zeta .
\end{aligned}
$$

Now combining this fact with the commutativity of the diagram

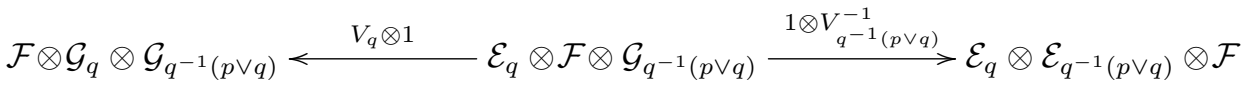

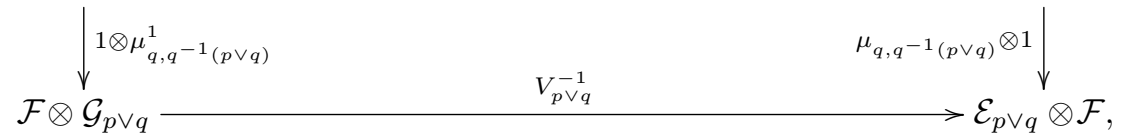

we deduce from the definition of the $U_{g}$ 's for $g \in P^{-1}$ that 5.2 .9 commutes if we let $p \vee q$ play the role of $p$.

Let us prove that $(5.2 .9)$ is also commutative when one replaces $q$ by $p \vee q$. In this case, we have that

$$
\mathcal{E}_{p}^{*} \otimes_{A} \mathcal{E}_{p \vee q} \otimes_{A} \mathcal{F}=\mathcal{E}_{p}^{*} \otimes_{A} \mathcal{E}_{p \vee q} \otimes_{A} \mathcal{F}\left\langle\mathcal{G}_{p \vee q} \mid \mathcal{G}_{p \vee q}\right\rangle
$$

and notice that, for $\xi_{1}, \xi_{2} \in \mathcal{G}_{p}$ and $\eta_{1}, \eta_{2} \in \mathcal{G}_{p^{-1}(p \vee q)}$, one has

$$
\left\langle\left\langle\mu_{p, p^{-1}(p \vee q)}^{1}\left(\xi_{1} \otimes \eta_{1}\right) \mid \mu_{p, p^{-1}(p \vee q)}^{1}\left(\xi_{2} \otimes \eta_{2}\right)\right\rangle\right\rangle=\left\langle\left\langle\xi_{1}\left\langle\left\langle\eta_{1} \mid \eta_{2}\right\rangle\right\rangle \mid \xi_{2}\right\rangle\right\rangle .
$$

We then establish the proof of our claim by applying the commutativity of the diagram

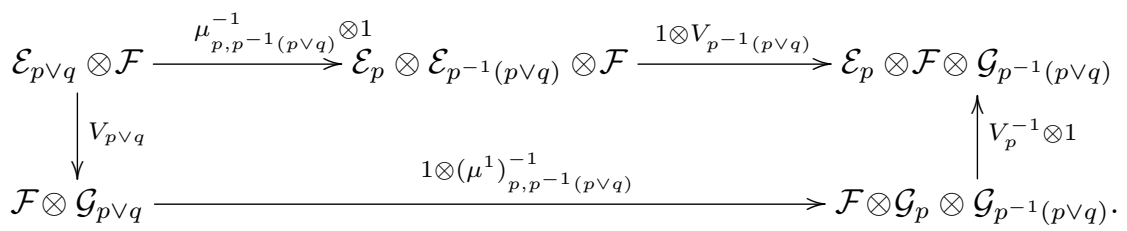

In order to prove the general case, we use the equality

$$
\langle\langle\xi \mid \eta\rangle\rangle \zeta=\xi\langle\eta \mid \zeta\rangle
$$

where $\xi, \eta, \zeta \in \mathcal{E}_{p \vee q}$. This implies the commutativity of the diagram

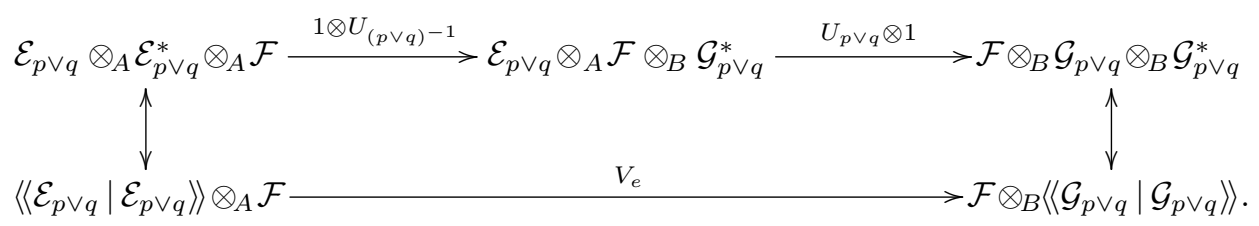

Then the commutativity of this diagram and of $(5.2 .9)$ for the above particular cases establish the commutativity of $(5.2 .9)$ for all $p, q \in P$.

We are left with the task of proving uniqueness of $U=\left\{U_{g}\right\}_{g \in G}$. This will follow from successive applications of the coherence axiom 5.2.5. Let $U^{\prime}=\left\{U_{g}^{\prime}\right\}_{g \in G}$ be a family of isometries making $\mathcal{F}$ into a correspondence

$$
\left(\hat{\mathcal{E}}_{g}\right)_{g \in G} \rightarrow\left(\hat{\mathcal{G}}_{g}\right)_{g \in G}
$$

and such that $U_{p}^{\prime}=V_{p}$ for all $p \in P$. By (5.2.5),

$$
U_{g}^{\prime}=\left(U_{g \vee e}^{\prime} \otimes 1\right)\left(1 \otimes U_{\left(g^{-1} \vee e\right)^{-1}}^{\prime}\right)=\left(V_{g \vee e} \otimes 1\right)\left(1 \otimes U_{\left(g^{-1} \vee e\right)^{-1}}^{\prime}\right) .
$$

Hence it suffices to show that $U_{p^{-1}}^{\prime}=U_{p^{-1}}$ for all $p \in P$. To do so, we use again 5.2 .5 to obtain the 


\section{FELL BUNDLES OVER QUASI-LATTICE ORDERED GROUPS}

commutative diagram

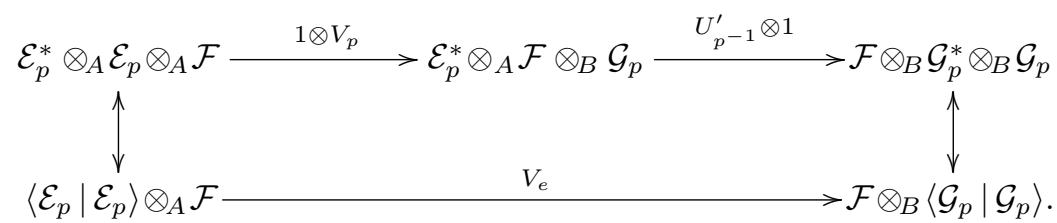

With the canonical identification can : $\mathcal{E}_{p}^{*} \otimes_{A} \mathcal{F} \cong \mathcal{E}_{p}^{*} \otimes_{A}\left\langle\left\langle\mathcal{E}_{p} \mid \mathcal{E}_{p}\right\rangle\right\rangle \otimes_{A} \mathcal{F}$, a commutative diagram as above for $\mathcal{E}_{p}^{*}$ and $\left\langle\left\langle\mathcal{E}_{p} \mid \mathcal{E}_{p}\right\rangle\right\rangle$ shows that

$$
U_{p^{-1}}^{\prime}=\left(U_{p^{-1}}^{\prime} \otimes 1_{B}\right)\left(1_{\mathcal{E}_{p}^{*}} \otimes V_{e}\right) \circ \text { can. }
$$

Since $V_{p}$ is unitary, we may replace the right-hand side above by

$$
\left(U_{p^{-1}}^{\prime} \otimes 1_{B}\right)\left(1_{\mathcal{E}_{p}^{*}} \otimes\left(V_{p} \otimes 1_{\mathcal{G}_{p}^{*}}\right)\left(V_{p}^{-1} \otimes 1_{\mathcal{G}_{p}^{*}}\right)\right)\left(1_{\mathcal{E}_{p}^{*}} \otimes V_{e}\right) \circ \text { can }
$$

Now we apply 5.2 .10 to the composite $\left(U_{p^{-1}}^{\prime} \otimes 1_{\mathcal{G}_{p}}\right)\left(1_{\mathcal{E}_{p}^{*}} \otimes V_{p}\right)$ and arrive at a description of $U_{p^{-1}}^{\prime}$ which is precisely the definition of $U_{p^{-1}}$.

We let $\mathfrak{C}_{*}^{P}$ be the full sub-bicategory of $\mathfrak{C}^{P}$ whose objects are triples $(A, \mathcal{E}, \mathcal{I})$, where $\mathcal{E}$ is a simplifiable product system of Hilbert bimodules and $\mathcal{I}$ is the family of Katsura's ideals for $\mathcal{E}$ as in the previous lemma. We will denote by $\mathfrak{C}_{\mathrm{pr}, *}^{P}$ the sub-bicategory of $\mathfrak{C}_{*}^{P}$ in which the arrows are proper covariant correspondences.

Theorem 5.2.11. There is an equivalence of bicategories $\mathfrak{C}_{*}^{P} \rightarrow \mathfrak{C}^{(G, P)}$ which sends an object $(A, \mathcal{E}, \mathcal{I})$ to the associated Fell bundle $\left(\hat{\mathcal{E}}_{g}\right)_{g \in G}$ extended from $P$. This restricts to an equivalence $\mathfrak{C}_{\mathrm{pr}, *}^{P} \rightarrow \mathfrak{C}_{\mathrm{pr}}^{(G, P)}$.

Proof. In order to describe a homomorphism of bicategories $\mathfrak{C}_{*}^{P} \rightarrow \mathfrak{C}^{(G, P)}$, let us first prove that a 2-morphism $w:\left(\mathcal{F}_{0}, V_{0}\right) \Rightarrow\left(\mathcal{F}_{1}, V_{1}\right)$ produces a 2-arrow $w^{\sharp}:\left(\mathcal{F}_{0}^{\sharp}, U_{0}\right) \Rightarrow\left(\mathcal{F}_{1}^{\sharp}, U_{1}\right)$ such that $w^{\sharp}=w$ as a correspondence isomorphism $\mathcal{F}_{0} \cong \mathcal{F}_{1}$. To do so, we need to show that $w$ makes the diagram

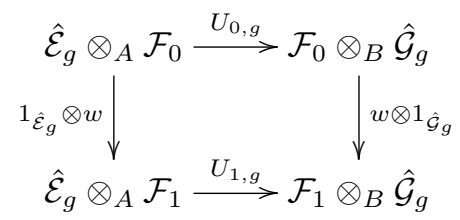

commute. By construction, this commutes for all $p \in P$. Hence it suffices to establish its commutativity for $g, h \in P^{-1}$, since $\left(\hat{\mathcal{E}}_{g}\right)_{g \in G}$ and $\left(\hat{\mathcal{G}}_{g}\right)_{g \in G}$ are extended from $P$. This follows from the commutativity of the diagram

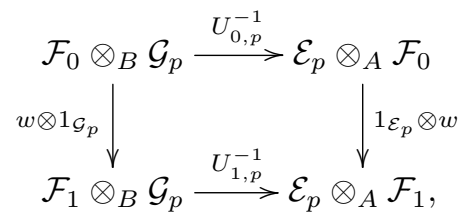

once $w$ intertwines the actions of $A$ and $B$. So we define a homomorphism of bicategories $L^{*}: \mathfrak{C}_{*}^{P} \rightarrow$ $\mathfrak{C}^{(G, P)}$ by sending a simplifiable product system $\mathcal{E}=\left(\mathcal{E}_{p}\right)_{p \in P}$ to its associated Fell bundle $\left(\hat{\mathcal{E}}_{g}\right)_{g \in G}$ and a morphism $(\mathcal{F}, V):\left(A, \mathcal{E}, \mathcal{I}_{\mathcal{E}}\right) \rightarrow\left(B, \mathcal{G}, \mathcal{I}_{\mathcal{G}}\right)$ to the arrow $\left(\mathcal{F}^{\sharp}, U\right):\left(\hat{\mathcal{E}}_{g}\right)_{g \in G} \rightarrow\left(\hat{\mathcal{G}}_{g}\right)_{g \in G}$ built out of $(\mathcal{F}, V)$ as in Lemma 5.2.8 A 2-arrow $w$ is mapped to $w^{\sharp}$ as above. Clearly, this data yields a functor

$$
\mathfrak{C}_{*}^{P}\left(\left(A, \mathcal{E}, \mathcal{I}_{\mathcal{E}}\right),\left(B, \mathcal{G}, \mathcal{I}_{\mathcal{G}}\right)\right) \rightarrow \mathfrak{C}^{(G, P)}\left(\left(\hat{\mathcal{E}}_{g}\right)_{g \in G},\left(\hat{\mathcal{G}}_{g}\right)_{g \in G}\right)
$$

between the groupoids of arrows associated to the objects $\left(A, \mathcal{E}, \mathcal{I}_{\mathcal{E}}\right)$ and $\left(B, \mathcal{G}, \mathcal{I}_{\mathcal{G}}\right)$. Furthermore, it follows from Lemmas 5.2.7 and 5.2.8 that such a functor is an equivalence. Given arrows

$$
(\mathcal{F}, V):\left(A, \mathcal{E}, \mathcal{I}_{\mathcal{E}}\right) \rightarrow\left(A_{1}, \mathcal{E}_{1}, \mathcal{I}_{\mathcal{E}_{1}}\right), \quad\left(\mathcal{F}_{1}, V_{1}\right):\left(A_{1}, \mathcal{E}_{1}, \mathcal{I}_{\mathcal{E}_{1}}\right) \rightarrow\left(A_{2}, \mathcal{E}_{2}, \mathcal{I}_{\mathcal{E}_{2}}\right)
$$


we have that

$$
\left(\mathcal{F} \otimes_{A_{1}} \mathcal{F}_{1}\right)^{\sharp}=\mathcal{F}^{\sharp} \otimes_{A_{1}} \mathcal{F}_{1}^{\sharp}=\mathcal{F} \otimes_{A_{1}} \mathcal{F}_{1}
$$

as correspondences $A \leadsto A_{2}$. Moreover, the product of arrows in $\mathfrak{C}^{(G, P)}$ is defined as in $\mathfrak{C}_{*}^{P}$ and Lemma 5.2.8 tells us that $\left(\mathcal{F} \otimes_{A_{1}} \mathcal{F}_{1}, V \bullet V_{1}\right)$ extends uniquely to a correspondence $\left(\hat{\mathcal{E}}_{g}\right)_{g \in G} \rightarrow\left(\hat{\mathcal{E}}_{2, g}\right)_{g \in G}$. This guarantees that $L^{*}$ preserves the product of arrows. Thus, this is indeed a homomorphism of bicategories.

As mentioned above, $L^{*}$ is locally an equivalence. So to see that $L^{*}$ is an equivalence, it remains to show that it is biessentially surjective by 28 , Lemma 3.1]. That is, for each $\left(B_{g}\right)_{g \in G} \in$ ob $\mathfrak{C}^{(G, P)}$, we must find $b \in \mathrm{ob}^{P}$ and arrows

$$
(\mathcal{F}, U): L^{*}(b) \rightarrow\left(B_{g}\right)_{g \in G}, \quad\left(\mathcal{F}^{\prime}, U^{\prime}\right):\left(B_{g}\right)_{g \in G} \rightarrow L^{*}(b)
$$

for which there are coherent isomorphisms

$$
(\mathcal{F}, U) \circ\left(\mathcal{F}^{\prime}, U^{\prime}\right) \cong\left(B_{e}, \hat{\iota}_{G}\right), \quad\left(\mathcal{F}^{\prime}, U^{\prime}\right) \circ(\mathcal{F}, U) \cong 1_{L^{*}(b)}
$$

Our natural choice of $b$ is the triple $\left(B_{e}, \mathcal{B}, \mathcal{I}_{\mathcal{B}}\right)$, where $\mathcal{B}$ is the product system of Hilbert bimodules associated to $\left(B_{g}\right)_{g \in G}$. This is simplifiable by Proposition 5.2.1 and hence it indeed gives rise to an object of $\mathfrak{C}_{*}^{P}$. So we let $\left(\hat{\mathcal{B}}_{g}\right)_{g \in G}$ be its image under $L^{*}$. Since the structure of product system of $\mathcal{B}$ is inherited from $\left(B_{g}\right)_{g \in G}$, Theorem 5.1 .8 implies that $\left(\hat{\mathcal{B}}_{g}\right)_{g \in G}$ is isomorphic to $\left(B_{g}\right)_{g \in G}$ in an obvious way. Such an isomorphism and the unit arrow $\left(B_{e}, \hat{\iota}_{G}\right)$, once put together, produce an adjoint equivalence between $\left(\hat{\mathcal{B}}_{g}\right)_{g \in G}$ and $\left(B_{g}\right)_{g \in G}$ (see 28 , Definition 1.1] for the required coherence axioms). The last assertion in the statement follows from the fact that $\mathcal{F}^{\sharp}=\mathcal{F}$ as correspondences over $A$.

\subsection{Amenability for Fell bundles extended from free semi- groups}

A quasi-lattice ordered group $(G, P)$ is called amenable if the Fock representation $\psi^{+}: \mathrm{C}^{*}(G, P) \rightarrow$ $\mathbb{B}\left(\ell_{2}(P)\right)$ is injective (see [47, Section 4.2] and also Example 4.2.13). Examples of amenable quasi-lattice orders are free groups 19 47, Baumslag-Solitar groups BS(c,d) with $c, d$ positive integers [16] and, of course, $(G, P)$ for an amenable group $G$. Counterexamples are, for instance, nonabelian Artin groups of finite type [17]. In [19], Exel proved that Fell bundles extended from a free semigroup $\mathbb{F}^{+}$are amenable, under a separability hypothesis. In this section, we follow the ideas of $[16$ to show that any Fell bundle extended from $\mathbb{F}^{+}$is amenable, with no extra assumptions. But here we deduce faithfulness of the regular representation from gauge-invariant uniqueness theorems for relative Cuntz-Pimsner algebras. The same techniques are employed to show that a Fell bundle extended from $\operatorname{BS}(c, d)^{+}$is always amenable. This suggests that amenability for Fell bundles extended from a positive cone is connected with amenability of the underlying quasi-lattice ordered group.

Proposition 5.3.1. A Fell bundle $\left(B_{g}\right)_{g \in \mathbb{F}}$ extended from $\mathbb{F}^{+}$is amenable, where $\mathbb{F}$ denotes the free group on a set of generators $S$.

Proof. Let $\theta: \mathbb{F} \rightarrow \mathbb{Z}$ be the group homomorphism defined on the generators by $a \mapsto 1$, for all $a \in S$. So for $b \in \mathbb{F}^{+}, \theta(b)=|b|$ is the length of $b$ in its reduced form. This induces a coaction of $\mathbb{Z}$ on $\left(B_{g}\right)_{g \in G}$ by [18, Example A.28]. Hence it provides $\mathrm{C}^{*}\left(\left(B_{g}\right)_{g \in \mathbb{F}}\right)$ with a topological $\mathbb{Z}$-grading, for which the corresponding spectral subspace at $m \in \mathbb{Z}$ is the closure of

$$
\operatorname{span}\left\{\xi_{p} \cdot \eta_{q}^{*} \mid p, q \in \mathbb{F}^{+} \text {and } \theta(p)-\theta(q)=m\right\} .
$$

Now let $\mathcal{G}$ be the direct sum $\bigoplus_{a \in S} B_{a}$ viewed as a correspondence over $B_{e}$ in the usual way. Let $I_{\mathcal{G}}$ be Katsura's ideal for $\mathcal{G}$. That is,

$$
I_{\mathcal{G}}=\varphi_{\mathcal{G}}^{-1}(\mathbb{K}(\mathcal{G})) \cap\left(\operatorname{ker} \varphi_{\mathcal{G}}\right)^{\perp}=\bigoplus_{a \in S} B_{a} B_{a}^{*}
$$




\section{FELL BUNDLES OVER QUASI-LATTICE ORDERED GROUPS}

This sum is indeed orthogonal because $B_{a} B_{a}^{*} B_{b} B_{b}^{*}=\{0\}$ for $a \neq b$. It follows that

$$
\left(\bigoplus_{a \in S} \xi_{a}\right)^{*}\left(\bigoplus_{a \in S} \eta_{a}\right)=\bigoplus_{a \in S} \xi_{a}^{*} \eta_{a}
$$

in $\mathrm{C}^{*}\left(\left(B_{g}\right)_{g \in \mathbb{F}}\right)$, where $\xi_{a}, \eta_{a} \in B_{a}$ for all $a \in S$. Thus we get a representation of $\mathcal{G}$ in $\mathrm{C}^{*}\left(\left(B_{g}\right)_{g \in \mathbb{F}}\right)$ obtained by restricting the representation of $\left(B_{g}\right)_{g \in G}$ to the $B_{a}$ 's. This is a gauge-compatible injective representation of $\mathcal{G}$ that is covariant on $I_{\mathcal{G}}$. Hence it induces an isomorphism $\mathcal{O}_{I_{\mathcal{G}}, \mathcal{G}} \rightarrow \mathrm{C}^{*}\left(\left(B_{g}\right)_{g \in \mathbb{F}}\right)$ by 29 , Theorem 6.4$]$.

We claim that $\mathrm{C}_{r}^{*}\left(\left(B_{g}\right)_{g \in \mathbb{F}}\right)$ also carries a topological $\mathbb{Z}$-grading, for which the regular representation $\Lambda: \mathrm{C}^{*}\left(\left(B_{g}\right)_{g \in \mathbb{F}}\right) \rightarrow \mathrm{C}_{r}^{*}\left(\left(B_{g}\right)_{g \in \mathbb{F}}\right)$ is a grading-preserving ${ }^{*}$-homomorphism. Indeed, for each $z \in \mathbb{T}$, define a unitary $U_{z} \in \mathbb{B}\left(\ell_{2}\left(\left(B_{g}\right)_{g \in \mathbb{F}}\right)\right)$ by setting

$$
\eta^{+}=\bigoplus_{g \in \mathbb{F}} \eta_{g} \mapsto U_{z}\left(\eta^{+}\right)=\bigoplus_{g \in \mathbb{F}} z^{\theta(g)} \eta_{g}
$$

Then $\Lambda(b) \mapsto U_{z} \Lambda(b) U_{z}^{*}$ is a continuous action of $\mathbb{T}$ on the reduced cross sectional $\mathrm{C}^{*}$-algebra of $\left(B_{g}\right)_{g \in \mathbb{F}}$. Hence $\mathrm{C}_{r}^{*}\left(\left(B_{g}\right)_{g \in \mathbb{F}}\right)$ is a topologically $\mathbb{Z}$-graded $\mathrm{C}^{*}$-algebra (see Example 2.2.8).

Thus the composition of the regular representation $\Lambda$ with the isomorphism $\mathcal{O}_{I_{\mathcal{G}}, \mathcal{G}} \cong \mathrm{C}^{*}\left(\left(B_{g}\right)_{g \in \mathbb{F}}\right)$ gives a gauge-compatible injective representation of $\mathcal{G}$ that is covariant on $I_{\mathcal{G}}$. So we invoke again the gauge-invariant uniqueness theorem for Katsura's relative Cuntz-Pimsner algebra of a single correspondence, namely $\left[29\right.$, Theorem 6.4], to derive faithfulness of $\Lambda$. This shows that $\left(B_{g}\right)_{g \in \mathbb{F}}$ is amenable.

Let $c$ and $d$ be positive integers. Recall from Example 4.2.4 that the Baumslag-Solitar group BS $(c, d)$ is the universal group on two generators $a$ and $b$ subject to the relation $a b^{c}=b^{d} a$ and $\left(\operatorname{BS}(c, d), \operatorname{BS}(c, d)^{+}\right)$ is a quasi-lattice ordered group, where $\mathrm{BS}(c, d)^{+}$is the unital subsemigroup generated by $a$ and $b$. As for free groups, there is a group homomorphism $\theta: \operatorname{BS}(c, d) \rightarrow \mathbb{Z}$ which is given on generators by $a \mapsto 1$ and $b \mapsto 0$. We follow $[16$ and $[56$ and call $\theta(g)$ for $g \in \operatorname{BS}(c, d)$ the height of $g$.

Each $p \in \operatorname{BS}(c, d)^{+}$has a reduced form

$$
p=b^{s_{0}} a b^{s_{1}} \ldots b^{s_{k-1}} a b^{s_{k}},
$$

with $0 \leq s_{i}<d$ for all $i \in\{1, \ldots, k-1\}$ and $\theta(p)=k$. As in [16], we set

$$
\operatorname{stem}(p):=b^{s_{0}} a b^{s_{1}} \ldots b^{s_{k-1}} a .
$$

Given a Fell bundle extended from $\operatorname{BS}(c, d)^{+}$, we will again construct a correspondence $\mathcal{G}$ over a $\mathrm{C}^{*}$-algebra $B$ so that $\mathcal{O}_{\mathcal{I}_{\mathcal{G}}, \mathcal{G}}$ is $\mathbb{Z}$-equivariantly isomorphic to $\mathrm{C}^{*}\left(\left(C_{g}\right)_{g \in B S(c, d)}\right)$.

We need the following lemma:

Lemma 5.3.2 ([16, Lemma 3.4]). Let $p, q \in \mathrm{BS}(c, d)^{+}$be such that $p \vee q<\infty$. Then,

(i) if $\theta(p)>\theta(q)$, there is $m \in \mathbb{N}$ with $p \vee q=p b^{m}$;

(ii) if $\theta(p)=\theta(q)$, there is $m \in \mathbb{N}$ with either

$$
p \vee q=p b^{m}=q, \quad \text { or } \quad p \vee q=q b^{m}=p .
$$

In particular, by the previous lemma, $p \vee q=\infty$ and hence $C_{p}^{*} C_{q}=\{0\}$ whenever $p$ and $q$ have reduced forms $b^{s_{0}} a$ and $b^{t_{0}} a$ with $s_{0} \neq t_{0}$.

Proposition 5.3.3. A Fell bundle $\left(C_{g}\right)_{g \in G}$ extended from $\mathrm{BS}(c, d)^{+}$is amenable.

Proof. Consider the $\mathrm{C}^{*}$-subalgebra $B$ of $\mathrm{C}^{*}\left(\left(C_{g}\right)_{g \in \mathrm{BS}(c, d)}\right)$ generated by the fibre $C_{b}$ and the unit fibre $C_{e}$. This is a topologically $\mathbb{Z}$-graded $\mathrm{C}^{*}$-algebra for which the conditional expectation onto $C_{e}$ coincides with that of $\mathrm{C}^{*}\left(\left(C_{g}\right)_{g \in \mathrm{BS}(c, d)}\right)$. The corresponding spectral subspace at $m \in \mathbb{Z}$ is $C_{b^{m}}$. We define a correspondence over $B$ as follows. For each $0 \leq i<d$, we let $\mathcal{G}_{i}=C_{b^{i} a} \otimes_{C_{e}} B$. We set

$$
\mathcal{G}=\bigoplus_{i=0}^{d-1} \mathcal{G}_{i}
$$


as a correspondence $C_{e} \leadsto B$. We extend the left action of $C_{e}$ to $B$ by using the multiplication in $B$. To do so, it suffices to find a representation of the Hilbert $C_{e}$-bimodule $C_{b}$ in $\mathbb{B}(\mathcal{G})$ that is Cuntz-Pimsner covariant on $C_{b} C_{b}^{*}$ by Proposition 3.1.26. Thus for $\xi \in C_{b}$ and $i+1<d$, take an elementary tensor $\eta \otimes \zeta \in \mathcal{G}_{i}$. We define

$$
\varphi_{\mathcal{G}_{i}}(\xi)(\eta \otimes \zeta):=(\xi \cdot \eta) \otimes \zeta \in \mathcal{G}_{i+1}
$$

If $i+1=d$, we use the relation $b^{d} a=a b^{c}$ and that $\left(C_{g}\right)_{g \in \operatorname{BS}(c, d)}$ is extended from $\operatorname{BS}(c, d)^{+}$to identify the multiplication $\xi \cdot \eta \otimes c$ with an element of $\mathcal{G}_{0}$. Notice that $a \vee b=b^{d} a=a b^{c}$ and hence $C_{b}^{*} C_{a} \subseteq C_{b^{d-1} a} C_{b^{c}}^{*}$. This guarantees that $\varphi_{\mathcal{G}}(\xi)$ is adjointable for all $\xi \in C_{b}$ and $\varphi_{\mathcal{G}}(\xi)^{*}$ is given in a similar way by multiplication with $\xi^{*}$. This produces a ${ }^{*}$-homomorphism $\varphi_{\mathcal{G}}: B \rightarrow \mathbb{B}(\mathcal{G})$, which turns $\mathcal{G}$ into a correspondence over $B$. Using the relation $b^{d} a=a b^{c}$ and also $a b^{-c}=b^{-d} a$, we deduce that $C_{b^{m}} C_{a} C_{a}^{*} C_{b^{n}}$ is contained in $C_{b^{i} a} \cdot B \cdot C_{b^{j} a}^{*}$ in $\mathrm{C}^{*}\left(\left(C_{g}\right)_{g \in F}\right)$, where $0 \leq i, j<d$ are uniquely determined by $m$ and $n$, respectively, and $m, n \in \mathbb{Z}$. From this we see that Katsura's ideal for $\mathcal{G}$ is

$$
I_{\mathcal{G}}=\overline{\operatorname{span}}\left\{C_{b^{m}} C_{a} C_{a}^{*} C_{b^{n}} \mid m, n \in \mathbb{Z}\right\} \triangleleft B,
$$

since the left action of $B$ on $\mathcal{G}$ involves the multiplication on $\left(C_{g}\right)_{g \in \mathrm{BS}(c, d)}$.

Because $C_{p}^{*} C_{q}=\{0\}$ whenever $p$ and $q$ have reduced forms $b^{s_{0}} a$ and $b^{t_{0}} a$ with $s_{0} \neq t_{0}$, we have a canonical representation of $\mathcal{G}$ in $\mathrm{C}^{*}\left(\left(C_{g}\right)_{g \in \mathrm{BS}(c, d)}\right)$ coming from the identification $\mathcal{G}_{i} \cong C_{b^{i} a} B$. Such a representation is injective, gauge-compatible and Cuntz-Pimsner covariant on $I_{\mathcal{G}}$. This gives a surjective *-homomorphism $\phi: \mathcal{O}_{I_{\mathcal{G}}, \mathcal{G}} \rightarrow \mathrm{C}^{*}\left(\left(C_{g}\right)_{g \in \mathrm{BS}(c, d)}\right)$ because $\left(C_{g}\right)_{g \in \mathrm{BS}(c, d)}$ is extended from the positive cone $\mathrm{BS}(c, d)^{+}$. Now $[29$, Theorem 6.4] shows that $\phi$ is an isomorphism. Employing the same argument used in Proposition 5.3.1. we conclude that $\mathrm{C}_{r}^{*}\left(\left(C_{g}\right)_{g \in \mathrm{BS}(c, d)}\right)$ also carries a topological $\mathbb{Z}$-grading, for which the regular representation is compatible. Thus $\Lambda: \mathrm{C}^{*}\left(\left(C_{g}\right)_{g \in \mathrm{BS}(c, d)}\right) \rightarrow \mathrm{C}_{r}^{*}\left(\left(C_{g}\right)_{g \in \mathrm{BS}(c, d)}\right)$ produces a gauge-compatible representation of $\mathcal{O}_{I_{\mathcal{G}}, \mathcal{G}}$ that is faithful on $B$, so that the gauge-invariant uniqueness theorem for $\mathcal{O}_{I_{\mathcal{G}}, \mathcal{G}}$ implies the desired isomorphism.

\subsection{Functoriality for relative Cuntz-Pimsner algebras}

Although a covariant correspondence between two objects of $\mathfrak{C}_{*}^{P}$ always produces a correspondence between the associated Fell bundles and vice-versa, we need properness to ensure that it will also induce a $\mathrm{C}^{*}$-correspondence between their $\mathrm{C}^{*}$-algebras. In this section, we will restrict our attention to proper covariant correspondences. The fact that a proper covariant correspondence between objects of $\mathfrak{C}_{\mathrm{pr}}^{P}$ yields a correspondence between their relative Cuntz-Pimsner algebras together with results from the previous section will imply that $\mathfrak{C}_{\mathrm{pr}, *}^{P}$ is a reflective sub-bicategory of $\mathfrak{C}_{\mathrm{pr}}^{P}$. We will then use functoriality for relative Cuntz-Pimsner algebras to study Morita equivalence between these $\mathrm{C}^{*}$-algebras, arising from equivalences in $\mathfrak{C}_{\mathrm{pr}}^{P}$.

\subsubsection{Relative Cuntz-Pimsner algebras as universal arrows}

We begin by constructing correspondences out of morphisms in $\mathfrak{C}_{\mathrm{pr}}^{P}$.

Proposition 5.4.1. Let $(\mathcal{F}, V):(A, \mathcal{E}, \mathcal{J}) \rightarrow\left(B, \mathcal{G}, \mathcal{J}_{B}\right)$ be a proper covariant correspondence. It induces a proper correspondence $\mathcal{O}_{\mathcal{F}, V}: \mathcal{O}_{\mathcal{J}_{A}, \mathcal{E}} \leadsto \mathcal{O}_{\mathcal{J}_{B}, \mathcal{G}}$. In particular, a morphism in $\mathfrak{C}_{\mathrm{pr}, *}^{P}$ between two simplifiable product systems of Hilbert bimodules produces a proper correspondence between the cross sectional $\mathrm{C}^{*}$-algebras of the associated Fell bundles.

Proof. Let $\mathcal{F}_{\mathcal{O}}:=\mathcal{F} \otimes_{B} \mathcal{O}_{\mathcal{J}_{B}, \mathcal{G}}$. We define a family of isometries $V^{!}=\left\{V_{p}^{!}\right\}_{p \in P}$ by setting, for all $p \in P$,

$$
V_{p}^{!}: \mathcal{E}_{p} \otimes_{A} \mathcal{F}_{\mathcal{O}}=\mathcal{E}_{p} \otimes_{A} \mathcal{F} \otimes_{B} \mathcal{O}_{\mathcal{J}_{B}, \mathcal{G}} \stackrel{V_{p} \otimes \mathrm{id}}{\Longrightarrow} \mathcal{F} \otimes_{B} \mathcal{G}_{p} \otimes_{B} \mathcal{O}_{\mathcal{J}_{B}, \mathcal{G}} \stackrel{\mathrm{id} \otimes \mu_{\mathcal{G}_{p}}}{\Longrightarrow} \mathcal{F}_{\mathcal{O}},
$$

where $\mu_{\mathcal{G}_{p}}$ is the isometry $\mathcal{G}_{p} \otimes_{B} \mathcal{O}_{\mathcal{J}_{B}, \mathcal{G}} \Rightarrow \mathcal{O}_{\mathcal{J}_{B}, \mathcal{G}}$ obtained from the representation of $\mathcal{G}_{p}$ in $\mathcal{O}_{\mathcal{J}_{B}, \mathcal{G}}$ For each $\xi \in \mathcal{E}_{p}$, we set

$$
\psi_{p}(\xi)(\eta):=V_{p}^{!}\left(\xi \otimes_{A} \eta\right), \quad \eta \in \mathcal{F}_{\mathcal{O}} .
$$

Because $\mathcal{F}$ and $\mathcal{O}_{\mathcal{J}_{B}, \mathcal{G}}$ are proper correspondences, the map $\eta \mapsto \xi \otimes_{A} \eta$ is compact. This is mapped to $\mathbb{K}\left(\mathcal{F}_{\mathcal{O}}\right)$ when composed with $V_{p}^{!}$and, in particular, $\psi_{p}(\xi)$ is adjointable. The coherence axiom 4.3.2 


\section{FELL BUNDLES OVER QUASI-LATTICE ORDERED GROUPS}

for $(\mathcal{F}, V)$ implies that $\psi=\left\{\psi_{p}\right\}_{p \in P}$ preserves the multiplication on $\mathcal{E}$. In addition, for all $\xi, \eta \in \mathcal{E}_{p}$ and $\zeta, \zeta^{\prime} \in \mathcal{F}_{\mathcal{O}}$, we have that

$$
\left\langle\psi_{p}(\xi)^{*} \psi_{p}(\eta) \zeta \mid \zeta^{\prime}\right\rangle=\left\langle\psi_{p}(\eta) \zeta \mid \psi_{p}(\xi) \zeta^{\prime}\right\rangle=\left\langle\zeta \mid \psi_{e}(\langle\eta \mid \xi\rangle) \zeta^{\prime}\right\rangle=\left\langle\psi_{e}(\langle\xi \mid \eta\rangle) \zeta \mid \zeta^{\prime}\right\rangle
$$

provided $V_{p}^{!}$is an isometry. Therefore, $\psi=\left\{\psi_{p}\right\}_{p \in P}$ is a representation of $\mathcal{E}$ by compact operators on $\mathcal{F}_{\mathcal{O}}$.

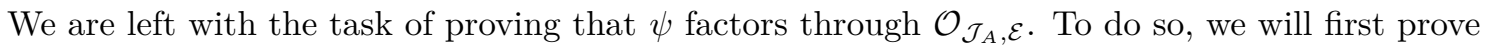
that it is Cuntz-Pimsner covariant on $\mathcal{J}_{A}=\left\{J_{p}^{A}\right\}_{p \in P}$. The Nica covariance condition will then follow from the fact that the $G$-grading of $\mathcal{O}_{\mathcal{J}_{B}, \mathcal{G}}$ is extended from $P$. The representation of $\mathcal{G}$ in $\mathcal{O}_{\mathcal{J}_{B}, \mathcal{G}}$ is covariant on $\mathcal{J}_{B}$. Hence the ${ }^{*}$-homomorphism $j_{J_{B}}: B \rightarrow \mathcal{O}_{\mathcal{J}_{B}, \mathcal{G}}$ satisfies

$$
j_{\mathcal{J}_{B}}\left(J_{p}^{B}\right) \mathcal{O}_{\mathcal{J}_{B}, \mathcal{G}} \subseteq \mu_{\mathcal{G}_{p}}\left(\mathcal{G}_{p} \otimes_{b} \mathcal{O}_{\mathcal{J}_{B}, \mathcal{G}}\right)
$$

for all $p \in P$. It follows that $\psi_{e}\left(J_{p}^{A}\right)$ maps $\mathcal{F}_{\mathcal{O}}$ into $\mathcal{F} \otimes_{B} \mu_{\mathcal{G}_{p}}\left(\mathcal{G}_{p} \otimes_{B} \mathcal{O}_{\mathcal{J}_{B}, \mathcal{G}}\right)$, provided $J_{p}^{A} \mathcal{F} \subseteq \mathcal{F} J_{p}^{B}$. Using that $V_{p}$ is unitary, we see that this coincides with $\psi_{p}\left(\mathcal{E}_{p}\right) \mathcal{F}_{\mathcal{O}}$. Proposition 3.1 .19 ensures that $\psi$ is covariant on $J_{p}^{A}$.

To see that $\psi$ is also Nica covariant, let $p, q \in P, T \in \mathbb{K}\left(\mathcal{E}_{p}\right)$ and $S \in \mathbb{K}\left(\mathcal{E}_{q}\right)$. Then

$$
\psi^{(p)}(T)\left(\mathcal{F}_{\mathcal{O}}\right) \subseteq \mathcal{F} \otimes_{B} \mu_{\mathcal{G}_{p}}\left(\mathcal{G}_{p} \otimes_{B} \mathcal{O}_{\mathcal{J}_{B}, \mathcal{G}}\right)
$$

and

$$
\psi^{(q)}(S)\left(\mathcal{F}_{\mathcal{O}}\right) \subseteq \mathcal{F} \otimes_{B} \mu_{\mathcal{G}_{q}}\left(\mathcal{G}_{q} \otimes_{B} \mathcal{O}_{\mathcal{J}_{B}, \mathcal{G}}\right) .
$$

We deduce that $\psi^{(p)}(T) \psi^{(q)}(S)=0$ if $p \vee q=\infty$ because

$$
\mu_{\mathcal{G}_{p}}\left(\mathcal{G}_{p} \otimes_{B} \mathcal{O}_{\mathcal{J}_{B}, \mathcal{G}}\right)^{*} \mu_{\mathcal{G}_{q}}\left(\mathcal{G}_{q} \otimes_{B} \mathcal{O}_{\mathcal{J}_{B}, \mathcal{G}}\right)=\{0\}
$$

in $\mathcal{O}_{\mathcal{J}_{B}, \mathcal{G}}$, so that $\left\langle\xi \mid \psi^{(p)}(T) \psi^{(q)}(S) \eta\right\rangle=0$ for all $\xi, \eta \in \mathcal{F}_{\mathcal{O}}$. In case $p \vee q<\infty$, we have that $\psi^{(p)}(T) \psi^{(q)}(S)$ maps $\mathcal{F}_{\mathcal{O}}$ into the intersection

$$
\left(\mathcal{F} \otimes_{B} \mu_{\mathcal{G}_{p}}\left(\mathcal{G}_{p} \otimes_{B} \mathcal{O}_{\mathcal{J}_{B}, \mathcal{G}}\right)\right) \cap\left(\mathcal{F} \otimes_{B} \mu_{\mathcal{G}_{q}}\left(\mathcal{G}_{q} \otimes_{B} \mathcal{O}_{\mathcal{J}_{B}, \mathcal{G}}\right)\right)
$$

Now the representation of $\mathcal{G}$ in $\mathcal{O}_{\mathcal{J}_{B}, \mathcal{G}}$ is Nica covariant and hence

$$
j_{\mathcal{J}_{B}}^{(p)}\left(\mathbb{K}\left(\mathcal{G}_{p}\right)\right) \mu_{\mathcal{G}_{q}}\left(\mathcal{G}_{q} \otimes_{B} \mathcal{O}_{\mathcal{J}_{B}, \mathcal{G}}\right) \subseteq \mu_{\mathcal{G}_{p \vee q}}\left(\mathcal{G}_{p \vee q} \otimes_{B} \mathcal{O}_{\mathcal{J}_{B}, \mathcal{G}}\right)
$$

In addition, any element $\eta$ of $\mathcal{F} \otimes_{B} \mu_{\mathcal{G}_{p}}\left(\mathcal{G}_{p} \otimes_{B} \mathcal{O}_{\mathcal{J}_{B}, \mathcal{G}}\right)$ is the $\operatorname{limit} \lim _{\lambda} S_{\lambda}(\eta)$ with $\left(S_{\lambda}\right)_{\lambda \in \Lambda}$ an approximate identity for $\mathbb{K}\left(\mathcal{F} \otimes_{B} j_{\mathcal{J}_{B}}^{(p)}\left(\mathbb{K}\left(\mathcal{G}_{p}\right)\right)\right)$. This gives us

$$
\psi^{(p)}(T) \psi^{(q)}(S) \mathcal{F}_{\mathcal{O}} \subseteq \mathcal{F} \otimes_{B} \mu_{\mathcal{G}_{p \vee q}}\left(\mathcal{G}_{p \vee q} \otimes_{B} \mathcal{O}_{\mathcal{J}_{B}, \mathcal{G}}\right)
$$

The right-hand side above is contained in $\psi_{p \vee q}\left(\mathcal{E}_{p \vee q}\right) \mathcal{F}_{\mathcal{O}}$, provided $V_{p \vee q}$ is unitary. So we may invoke Proposition 3.1.19 again to deduce that $\psi$ is Nica covariant and therefore descends to a *-homomorphism $\mathcal{O}_{\mathcal{J}_{A}, \mathcal{E}} \rightarrow \mathbb{K}\left(\mathcal{F}_{\mathcal{O}}\right)$, as desired. The last assertion in the statement follows from the fact that $\mathrm{C}^{*}\left(\left(\hat{\mathcal{E}}_{g}\right)_{g \in G}\right)$ is canonically isomorphic to $\mathcal{O}_{\mathcal{I}_{\mathcal{E}}, \mathcal{E}}$ whenever $\mathcal{E}$ is a simplifiable product system of Hilbert bimodules (see Proposition 5.2.1.

By Corollary $5.2 .2\left(\mathcal{O}_{\mathcal{J}, \mathcal{E}}^{e},\left(\mathcal{O}_{\mathcal{J}, \mathcal{E}}^{p}\right)_{p \in P}, \mathcal{I}_{\mathcal{O}_{\mathcal{J}, \mathcal{E}}}\right)$ is an object of $\mathfrak{C}_{\mathrm{pr}, *}^{P}$ for each $(A, \mathcal{E}, \mathcal{J}) \in$ ob $\mathfrak{C}_{\mathrm{pr}}^{P}$. In what follows, we let

$$
v_{(A, \mathcal{E}, \mathcal{J})}:(A, \mathcal{E}, \mathcal{J}) \rightarrow\left(\mathcal{O}_{\mathcal{J}, \mathcal{E}}^{e},\left(\mathcal{O}_{\mathcal{J}, \mathcal{E}}^{p}\right)_{p \in P}, \mathcal{I}_{\mathcal{O}_{\mathcal{J}, \mathcal{E}}}\right)
$$

be the canonical proper covariance correspondence from Example 4.3 .4 That is, $v_{(A, \mathcal{E}, \mathcal{J})}:=\left(\mathcal{O}_{\mathcal{J}, \mathcal{E}}^{e}, \hat{\iota}_{\mathcal{E}}\right)$.

Proposition 5.4.2. Let $(A, \mathcal{E}, \mathcal{J})$ and $\left(B, \mathcal{G}, \mathcal{I}_{\mathcal{G}}\right)$ be objects of $\mathfrak{C}_{\mathrm{pr}}^{P}$ and $\mathfrak{C}_{\mathrm{pr}, *}^{P}$, respectively. There is a groupoid equivalence

$$
\mathfrak{C}_{\mathrm{pr}, *}^{P}\left(\left(\mathcal{O}_{\mathcal{J}, \mathcal{E}}^{e},\left(\mathcal{O}_{\mathcal{J}, \mathcal{E}}^{p}\right)_{p \in P}, \mathcal{I}_{\mathcal{O}_{\mathcal{J}, \mathcal{E}}}\right),\left(B, \mathcal{G}, \mathcal{I}_{\mathcal{G}}\right)\right) \cong \mathfrak{C}_{\mathrm{pr}}^{P}\left((A, \mathcal{E}, \mathcal{J}),\left(B, \mathcal{G}, \mathcal{I}_{\mathcal{G}}\right)\right)
$$

which is defined by composing objects with $v_{(A, \mathcal{E}, \mathcal{J})}$. 
Proof. Let $(\mathcal{F}, V):(A, \mathcal{E}, \mathcal{J}) \rightarrow\left(B, \mathcal{G}, \mathcal{I}_{\mathcal{G}}\right)$ be a morphism in $\mathfrak{C}_{\mathrm{pr}}^{P}$. Let $\mathcal{O}_{\mathcal{F}, V}$ be the correspondence $\mathcal{O}_{\mathcal{J}, \mathcal{E}} \leadsto \mathcal{O}_{\mathcal{I}_{\mathcal{G}}, \mathcal{G}}$ induced by $(\mathcal{F}, V)$ built in the previous proposition. By Proposition 5.1.18, $\mathcal{O}_{\mathcal{I}_{\mathcal{G}}, \mathcal{G}}^{e}$ is isomorphic to $B$ and hence $\mathcal{O}_{\mathcal{F}, V}^{e}=\mathcal{F} \otimes_{B} \mathcal{O}_{\mathcal{I}_{\mathcal{G}}, \mathcal{G}}^{e} \cong \mathcal{F}$. Since $\mathcal{O}_{\mathcal{J}, \mathcal{E}}^{e}$ acts by $G$-grading-preserving operators on $\mathcal{O}_{\mathcal{F}, V}$, this induces a nondegenerate ${ }^{*}$-homomorphism from $\mathcal{O}_{\mathcal{J}, \mathcal{E}}^{e}$ to $\mathbb{K}\left(\mathcal{F} \otimes_{B} \mathcal{O}_{\mathcal{I}_{\mathcal{G}}, \mathcal{G}}^{e} \cong \mathbb{K}(\mathcal{F})\right.$. This makes $\mathcal{F}$ into a proper correspondence $\mathcal{O}_{\mathcal{J}, \mathcal{E}}^{e} \leadsto B$, which we denote by $\mathcal{F}^{\sharp}$. We also have a correspondence isomorphism

$$
V_{p}^{\sharp}: \mathcal{O}_{\mathcal{J}, \mathcal{E}}^{p} \otimes_{\mathcal{O}_{\mathcal{J}, \mathcal{E}}^{e}} \mathcal{F}^{\sharp} \cong \mathcal{F}^{\sharp} \otimes_{B} \mathcal{G}_{p}
$$

obtained from $\mathcal{O}_{\mathcal{F}, V}$ because $\mathcal{O}_{\mathcal{F}, V}^{p}=\mathcal{F} \otimes_{B} \mathcal{O}_{\mathcal{I}_{\mathcal{G}}, \mathcal{G}}^{p} \cong \mathcal{F} \otimes \mathcal{G}_{p}$ for all $p \in P$ and $\mathcal{O}_{\mathcal{J}, \mathcal{E}}^{p} \mathcal{O}_{\mathcal{F}, V}^{e}=\mathcal{O}_{\mathcal{F}, V}^{p}$. It is indeed unitary, since

$$
\mathcal{F} \otimes_{B} \mathcal{G}_{p} \stackrel{u^{*}}{\cong} \mathcal{E}_{p} \otimes_{A} \mathcal{F} \cong \mathcal{E}_{p} \otimes_{A} \mathcal{O}_{\mathcal{J}, \mathcal{E}}^{e} \otimes_{\mathcal{O}_{\mathcal{J}, \mathcal{E}}^{e}} \mathcal{F}^{\sharp} \cong \mathcal{O}_{\mathcal{J}, \mathcal{E}}^{p} \otimes_{\mathcal{O}_{\mathcal{J}, \mathcal{E}}^{e}} \mathcal{F}^{\sharp}
$$

Thus we let $V^{\sharp}=\left\{V_{p}^{\sharp}\right\}_{p \in P}$.

In order to see that the pair $\left(\mathcal{F}^{\sharp}, V^{\sharp}\right)$ is a proper covariant correspondence

$$
\left(\mathcal{O}_{\mathcal{J}, \mathcal{E}}^{e},\left(\mathcal{O}_{\mathcal{J}, \mathcal{E}}^{p}\right)_{p \in P}, \mathcal{I}_{\mathcal{O}_{\mathcal{J}, \mathcal{E}}}\right) \rightarrow\left(B, \mathcal{G}, \mathcal{I}_{\mathcal{G}}\right)
$$

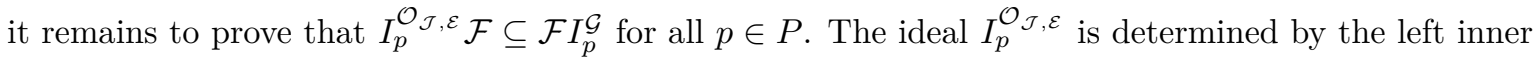
product, so that $I_{p}^{\mathcal{O}_{\mathcal{J}, \mathcal{E}}}=\mathcal{O}_{\mathcal{J}, \mathcal{E}}^{p} \mathcal{O}_{\mathcal{J}, \mathcal{E}}^{p}$. Now $\mathcal{O}_{\mathcal{J}, \mathcal{E}}^{p *}$ sends $\mathcal{O}_{\mathcal{F}, V}^{e}$ to $\mathcal{O}_{\mathcal{F}, V}^{p^{-1}}=\mathcal{F} \otimes_{B} \mathcal{O}_{\mathcal{I}_{\mathcal{G}}, \mathcal{G}}^{p} \cong \mathcal{F} \otimes_{B} \mathcal{G}_{p}^{*}$, while $\mathcal{O}_{\mathcal{J}, \mathcal{E}}^{p}$ maps $\mathcal{F} \otimes_{B} \mathcal{O}_{\mathcal{G}}^{p}{ }^{*}$ into $\mathcal{F} \otimes_{B} \mathcal{O}_{\mathcal{I}_{\mathcal{G}}, \mathcal{G}}^{p} \mathcal{O}_{\mathcal{I}_{\mathcal{G}}, \mathcal{G}}^{p}$. The isomorphism $\mathcal{O}_{\mathcal{I}_{\mathcal{G}}, \mathcal{G}}^{p} \mathcal{O}_{\mathcal{I}_{\mathcal{G}}, \mathcal{G}}^{p} \cong\left\langle\left\langle\mathcal{G}_{p} \mid \mathcal{G}_{p}\right\rangle\right\rangle$ implies that $\left(\mathcal{F}^{\sharp}, V^{\sharp}\right)$ is a proper covariant correspondence.

Now let $(\mathcal{F}, V):\left(\mathcal{O}_{\mathcal{J}, \mathcal{E}}^{e},\left(\mathcal{O}_{\mathcal{J}, \mathcal{E}}^{p}\right)_{p \in P}, \mathcal{I}_{\mathcal{O}_{\mathcal{J}, \mathcal{E}}}\right) \rightarrow\left(B, \mathcal{G}, \mathcal{I}_{\mathcal{G}}\right)$ be a proper covariant correspondence. Let $\mathcal{O}_{\mathcal{F}, V}$ be the proper correspondence $\mathcal{O}_{\mathcal{O}_{\mathcal{J}, \mathcal{E}}, \mathcal{I}_{\mathcal{O}}, \mathcal{E}} \leadsto \mathcal{O}_{\mathcal{I}_{\mathcal{G}}, \mathcal{G}}$ induced by $(\mathcal{F}, V)$ as in Proposition 5.4.1 As before, we have that $\mathcal{O}_{\mathcal{F}, V}^{p} \cong \mathcal{F} \otimes_{B} \mathcal{G}_{p}$ for all $p \in P$ and $\mathcal{O}_{\mathcal{F}, V}^{e} \cong \mathcal{F}$. By the same argument, we have isomorphisms $\mathcal{O}_{\mathcal{O}_{\mathcal{J}, \mathcal{E}}, \mathcal{I}_{\mathcal{O}}, \mathcal{E}}^{e} \cong \mathcal{O}_{\mathcal{J}, \mathcal{E}}^{e}$ and $\mathcal{O}_{\mathcal{O}_{\mathcal{J}, \mathcal{E}}, \mathcal{I}_{\mathcal{O}}, \mathcal{J}}^{p} \cong \mathcal{O}_{\mathcal{J}, \mathcal{E}}^{p}$. The restriction of the left action of $\mathcal{O}_{\mathcal{J}, \mathcal{E}}^{e}$ to $\mathcal{O}_{\mathcal{F}, V}^{p}$ is precisely its left action on $\mathcal{F} \otimes_{B} \mathcal{G}_{p}$. So we obtain a proper correspondence $\mathcal{F}^{b}: A \leadsto B$. We define isomorphisms $V_{p}^{b}: \mathcal{E}_{p} \otimes_{A} \mathcal{F}^{b} \cong \mathcal{F}^{b} \otimes_{B} \mathcal{G}_{p}$ using that $\mathcal{O}_{\mathcal{J}, \mathcal{E}}^{p}=j_{p}\left(\mathcal{E}_{p}\right) \mathcal{O}_{\mathcal{J}, \mathcal{E}}^{e}$ in $\mathcal{O}_{\mathcal{J}, \mathcal{E}}$ and $\mathcal{O}_{\mathcal{F}, V}^{e}$ is invariant under the left action of $\mathcal{O}_{\mathcal{J}, \mathcal{E}}^{e}$, so that

$$
\mathcal{E}_{p} \otimes_{A} \mathcal{F}^{b} \cong \mathcal{E}_{p} \otimes_{A} \mathcal{O}_{\mathcal{J}, \mathcal{E}}^{e} \otimes_{\mathcal{O}_{\mathcal{J}, \mathcal{E}}^{p}} \mathcal{F} \cong \mathcal{O}_{\mathcal{J}, \mathcal{E}}^{p} \otimes_{\mathcal{O}_{\mathcal{J}, \mathcal{E}}^{e}} \mathcal{F} \cong \mathcal{F}^{b} \otimes_{B} \mathcal{G}_{p}
$$

This gives a proper covariant correspondence $\left(\mathcal{F}^{b}, V^{b}\right):(A, \mathcal{E}, \mathcal{J}) \rightarrow\left(B, \mathcal{G}, \mathcal{I}_{\mathcal{G}}\right)$, since $J_{p}$ is sent to $I_{p}^{\mathcal{O}_{\mathcal{J}, \mathcal{E}}}$ for all $p \in P$ through the canonical *-homomorphism $A \rightarrow \mathcal{O}_{\mathcal{J}, \mathcal{E}}^{e}$.

A representation of $\mathcal{O}_{\mathcal{J}, \mathcal{E}}$ is uniquely determined by $(A, \mathcal{E})$. So, by construction, $\left(\mathcal{F}^{\sharp b}, V^{\sharp b}\right)=(\mathcal{F}, V)$ for a given morphism $(\mathcal{F}, V):(A, \mathcal{E}, \mathcal{J}) \rightarrow\left(B, \mathcal{G}, \mathcal{I}_{\mathcal{G}}\right)$. Similarly, we have $\left(\mathcal{F}^{b \sharp}, V^{b \sharp}\right)=(\mathcal{F}, V)$ for a proper covariant correspondence $(\mathcal{F}, V):\left(\mathcal{O}_{\mathcal{J}, \mathcal{E}}^{e},\left(\mathcal{O}_{\mathcal{J}, \mathcal{E}}^{p}\right)_{p \in P}, \mathcal{I}_{\mathcal{O}_{\mathcal{J}, \mathcal{E}}}\right) \rightarrow\left(B, \mathcal{G}, \mathcal{I}_{\mathcal{G}}\right)$. Therefore, $(\mathcal{F}, V) \mapsto$ $\left(\mathcal{F}^{b}, V^{b}\right)$ is a one-to-one correspondence.

We claim that a 2-arrow $w:\left(\mathcal{F}_{0}, V_{0}\right) \rightarrow\left(\mathcal{F}_{1}, V_{1}\right)$ is also $\mathcal{O}_{\mathcal{J}, \mathcal{E}^{-}}^{e}$ linear regarded as an isomorphism $\mathcal{F}_{0}^{\sharp} \cong$ $\mathcal{F}_{1}^{\sharp}$. Indeed, let $\operatorname{Ad}_{w \otimes 1}: \mathbb{B}\left(\mathcal{F}_{0, \mathcal{O}}\right) \rightarrow \mathbb{B}\left(\mathcal{F}_{1, \mathcal{O}}\right)$ be given by

$$
T \mapsto\left(w \otimes 1_{\mathcal{O}_{\mathcal{G}, \mathcal{I}_{\mathcal{G}}}}\right) T\left(w^{*} \otimes 1_{\mathcal{O}_{\mathcal{G}, \mathcal{I}_{\mathcal{G}}}}\right) .
$$

Composing it with the representation of $\mathcal{E}$ in $\mathbb{K}\left(\mathcal{F}_{0, \mathcal{O}}\right)$, we get a Nica covariant representation of $\mathcal{E}$ in $\mathbb{K}\left(\mathcal{F}_{1, \mathcal{O}}\right)$ that is covariant on $\mathcal{J}$ and coincides with its representation in $\mathbb{K}\left(\mathcal{F}_{1, \mathcal{O}}\right)$ obtained as in Proposition 5.4.1. Since a representation of $\mathcal{O}_{\mathcal{J}, \mathcal{E}}^{e}$ is uniquely determined by its restriction to $A$ and $\mathcal{E}$, we conclude that $w$ is also $\mathcal{O}_{\mathcal{J}, \mathcal{E}}^{e}$-linear. From this we deduce that $w$ satisfies the coherence axiom needed for a 2-morphism

$$
\left(\mathcal{F}_{0}^{\sharp}, V_{0}^{\sharp}\right) \Rightarrow\left(\mathcal{F}_{1}^{\sharp}, V_{1}^{\sharp}\right) .
$$

We denote the corresponding 2 -arrow by $w^{\sharp}$. We also let $w^{b}$ be the 2 -arrow in $\mathfrak{C}_{\mathrm{pr}}^{P}\left((A, \mathcal{E}, \mathcal{J}),\left(B, \mathcal{G}, \mathcal{I}_{\mathcal{G}}\right)\right)$ obtained from a 2-arrow in $\mathfrak{C}_{\mathrm{pr}, *}^{P}\left(\left(\mathcal{O}_{\mathcal{J}, \mathcal{E}}^{e},\left(\mathcal{O}_{\mathcal{J}, \mathcal{E}}^{p}\right)_{p \in P}, \mathcal{I}_{\mathcal{O}_{\mathcal{J}, \mathcal{E}}}\right),\left(B, \mathcal{G}, \mathcal{I}_{\mathcal{G}}\right)\right)$. Then $(\mathcal{F}, V) \mapsto\left(\mathcal{F}^{b}, V^{b}\right)$, $w \mapsto w^{b}$ is an equivalence of categories. This functor is naturally equivalent to the one defined by composition with $v_{(A, \mathcal{E}, \mathcal{J})}$. Such an equivalence has component at $(\mathcal{F}, V)$ determined by the canonical correspondence isomorphism $\mathcal{O}_{\mathcal{J}, \mathcal{E}}^{e} \otimes_{\mathcal{O}_{\mathcal{J}, \mathcal{E}}^{e}} \mathcal{F} \cong \mathcal{F}^{b}$. Therefore, composition with $v_{(A, \mathcal{E}, \mathcal{J})}$ establishes a 


\section{FELL BUNDLES OVER QUASI-LATTICE ORDERED GROUPS}

groupoid equivalence.

Corollary 5.4.3. The sub-bicategory $\mathfrak{C}_{\mathrm{pr}, *}^{P} \subseteq \mathfrak{C}_{\mathrm{pr}}^{P}$ is reflective. That is, the inclusion homomorphism $R: \mathfrak{C}_{\mathrm{pr}, *}^{P} \hookrightarrow \mathfrak{C}_{\mathrm{pr}}^{P}$ has a left adjoint $L: \mathfrak{C}_{\mathrm{pr}}^{P} \rightarrow \mathfrak{C}_{\mathrm{pr}, *}^{P}$. This is defined on objects by

$$
(A, \mathcal{E}, \mathcal{J}) \mapsto\left(\mathcal{O}_{\mathcal{J}, \mathcal{E}}^{e},\left(\mathcal{O}_{\mathcal{J}, \mathcal{E}}^{p}\right)_{p \in P}, \mathcal{I}_{\mathcal{O}_{\mathcal{J}, \mathcal{E}}}\right)
$$

Proof. By Proposition 5.4.2 $v_{(A, \mathcal{E}, \mathcal{J})}:(A, \mathcal{E}, \mathcal{J}) \rightarrow\left(\mathcal{O}_{\mathcal{J}, \mathcal{E}}^{e},\left(\mathcal{O}_{\mathcal{J}, \mathcal{E}}^{p}\right)_{p \in P}, \mathcal{I}_{\mathcal{O}_{\mathcal{J}, \mathcal{E}}}\right)$ is a universal arrow. We conclude that $R$ is left adjointable by Theorem 3.3.5. From the proof of the theorem, we deduce that its left adjoint $L$ sends $(A, \mathcal{E}, \mathcal{J})$ to $\left(\mathcal{O}_{\mathcal{J}, \mathcal{E}}^{e},\left(\mathcal{O}_{\mathcal{J}, \mathcal{E}}^{p}\right)_{p \in P}, \mathcal{I}_{\mathcal{O}_{\mathcal{J}, \mathcal{E}}}\right)$.

The homomorphism $L$ maps an arrow $(\mathcal{F}, V):(A, \mathcal{E}, \mathcal{J}) \rightarrow\left(A_{1}, \mathcal{E}_{1}, \mathcal{J}_{1}\right)$ to

$$
L(\mathcal{F}, V)=\left(v_{\left(A_{1}, \mathcal{E}_{1}, \mathcal{J}_{1}\right)} \circ(\mathcal{F}, V)\right)^{\sharp}=\left(\left(\mathcal{F} \otimes_{A_{1}} \mathcal{O}_{\mathcal{J}_{1}, \mathcal{E}_{1}}^{e}\right)^{\sharp},\left(V \bullet \bar{\iota}_{\mathcal{E}_{1}}\right)^{\sharp}\right) .
$$

It is defined on a 2-arrow $w:\left(\mathcal{F}_{0}, V_{0}\right) \Rightarrow\left(\mathcal{F}_{1}, V_{1}\right)$ by $L(w)=\left(w \otimes 1_{\mathcal{O}_{\mathcal{J}_{1}, \mathcal{E}_{1}}}\right)^{\sharp}$.

Let $(\mathcal{F}, V):(A, \mathcal{E}, \mathcal{J}) \rightarrow\left(A_{1}, \mathcal{E}_{1}, \mathcal{J}_{1}\right)$ and $\left(\mathcal{F}_{1}, V_{1}\right):\left(A_{1}, \mathcal{E}_{1}, \mathcal{J}_{1}\right) \rightarrow\left(A_{2}, \mathcal{E}_{2}, \mathcal{J}_{2}\right)$ be proper covariant correspondences. The isomorphism

$$
\lambda\left((\mathcal{F}, V),\left(\mathcal{F}_{1}, V_{1}\right)\right): L\left(\mathcal{F}_{1}, V_{1}\right) \circ L(\mathcal{F}, V) \cong L\left(\left(\mathcal{F}_{1}, V_{1}\right) \circ(\mathcal{F}, V)\right)
$$

is built out of the left action of $\mathcal{O}_{\mathcal{J}_{1}, \mathcal{E}_{1}}^{e}$ on $\left(\mathcal{F}_{1} \otimes_{A_{2}} \mathcal{O}_{\mathcal{J}_{2}, \mathcal{E}_{2}}^{e}\right)^{\sharp}$ constructed in Proposition 5.4.2. That is, it is given by the canonical isomorphism

$$
\left(\mathcal{F} \otimes_{A_{1}} \mathcal{O}_{\mathcal{J}_{1}, \mathcal{E}_{1}}^{e}\right)^{\sharp} \otimes_{\mathcal{O}_{\mathcal{J}_{1}, \mathcal{E}_{1}}^{e}}\left(\mathcal{F}_{1} \otimes_{A_{2}} \mathcal{O}_{\mathcal{J}_{2}, \mathcal{E}_{2}}^{e}\right)^{\sharp} \cong\left(\mathcal{F} \otimes_{A_{1}} \mathcal{F}_{1} \otimes_{A_{2}} \mathcal{O}_{\mathcal{J}_{2}, \mathcal{E}_{2}}^{e}\right)^{\sharp} .
$$

The compatibility isomorphism for units is obtained from the nondegenerate ${ }^{*}$-homomorphism $j_{\mathcal{J}}: A \rightarrow$ $\mathcal{O}_{\mathcal{J}, \mathcal{E}}^{e}$

\subsubsection{Morita equivalence for relative Cuntz-Pimsner algebras}

Let $\mathfrak{C}^{G}$ denote the bicategory whose objects are $\mathrm{C}^{*}$-algebras carrying a coaction of $G$. Arrows are correspondences with a coaction of $G$ compatible with those on the underlying $\mathrm{C}^{*}$-algebras. We refer to [18, Definition 2.10] for a precise definition. See also [18, Theorem 2.15] for $\mathfrak{C}^{G}$. Let $\mathfrak{C}_{\mathrm{pr}}^{G}$ be the sub-bicategory of $\mathfrak{C}^{G}$ whose arrows are proper correspondences.

Corollary 5.4.4. The construction of relative Cuntz-Pimsner algebras is functorial. There is a homomorphism of bicategories $\mathfrak{C}_{\mathrm{pr}}^{P} \rightarrow \mathfrak{C}_{\mathrm{pr}}^{G}$ which is defined on objects by

$$
(A, \mathcal{E}, \mathcal{J}) \mapsto \mathcal{O}_{\mathcal{J}, \mathcal{E}}
$$

Proof. It follows from Proposition 5.4.1 that a proper covariant correspondence between two simplifiable product systems of Hilbert bimodules gives rise to a nondegenerate proper correspondence between their relative Cuntz-Pimsner algebras with a gauge-compatible coaction of $G$. This yields a homomorphism of bicategories $\mathfrak{C}_{\mathrm{pr}, *}^{P} \rightarrow \mathfrak{C}_{\mathrm{pr}}^{G}$ which sends $\left(B, \mathcal{G}, \mathcal{I}_{\mathcal{G}}\right)$ to $\mathcal{O}_{\mathcal{I}_{\mathcal{G}}, \mathcal{G}}$ and a proper covariant correspondence $(\mathcal{F}, V):\left(B, \mathcal{G}, \mathcal{I}_{\mathcal{G}}\right) \rightarrow\left(B_{1}, \mathcal{G}_{1}, \mathcal{I}_{\mathcal{G}_{1}}\right)$ to $\mathcal{O}_{\mathcal{F}, V}$. Composing such a homomorphism with the reflector obtained in Corollary 5.4 .3 we obtain a homomorphism $\mathfrak{C}_{\mathrm{pr}}^{P} \rightarrow \mathfrak{C}^{\mathrm{G}}$.

By Proposition $5.2 .1, \mathcal{O}_{\mathcal{I}_{\mathcal{G}}, \mathcal{G}}$ is naturally isomorphic to the cross sectional $\mathrm{C}^{*}$-algebra of the associated Fell bundle extended from $P$. This establishes a canonical isomorphism

$$
\mathcal{O}_{\mathcal{O}_{\mathcal{J}, \mathcal{E}}, \mathcal{I}_{\mathcal{O}, \mathcal{E}}} \cong \mathcal{O}_{\mathcal{J}, \mathcal{E}}
$$

because $\mathcal{O}_{\mathcal{J}, \mathcal{E}}$ is isomorphic to the cross sectional $\mathrm{C}^{*}$-algebra of the Fell bundle associated to the gauge coaction of $G$. Here $\mathcal{O}_{\mathcal{O}_{\mathcal{J}, \mathcal{E}}, \mathcal{I}_{\mathcal{O}}, \mathcal{E}}$ is the relative Cuntz-Pimsner algebra for Katsura's ideals of $\left(\mathcal{O}_{\mathcal{J}, \mathcal{E}}^{p}\right)_{p \in P}$. From this we obtain a homomorphism of bicategories $\mathfrak{C}_{\mathrm{pr}}^{P} \rightarrow \mathfrak{C}^{G}$ which maps $(A, \mathcal{E}, \mathcal{J})$ to $\mathcal{O}_{\mathcal{J}, \mathcal{E}}$ and a proper covariant correspondence $(\mathcal{F}, V):(A, \mathcal{E}, \mathcal{J}) \rightarrow\left(A_{1}, \mathcal{E}_{1}, \mathcal{J}_{1}\right)$ to $\mathcal{O}_{\mathcal{F}, V}$. By construction, such a homomorphism is naturally equivalent to the one described in the previous paragraph.

Corollary 5.4.5. Let $(A, \mathcal{E}, \mathcal{J})$ and $\left(B, \mathcal{G}, \mathcal{J}_{B}\right)$ be objects of $\mathfrak{C}_{\mathrm{pr}}^{P}$. Then $\mathcal{O}_{\mathcal{J}, \mathcal{E}}$ and $\mathcal{O}_{\mathcal{J}_{B}, \mathcal{G}}$ are Morita equivalent if there is a covariant correspondence $(\mathcal{F}, V):(A, \mathcal{E}, \mathcal{J}) \rightarrow\left(B, \mathcal{G}, \mathcal{J}_{B}\right)$ so that $J_{p}^{A} \mathcal{F}=\mathcal{F} J_{p}^{B}$ 
for all $p \in P$ and $\mathcal{F}: A \leadsto B$ establishes a Morita equivalence. For objects in $\mathfrak{C}_{\mathrm{pr}, *}^{P}$, this equivalence preserves amenability of Fell bundles.

Proof. First, notice that $\mathcal{F}$ is automatically a proper correspondence. By Corollary 5.4.4 and Lemma 3.1.9, it suffices to show that $\mathcal{F}$ is an invertible arrow in $\mathfrak{C}_{\mathrm{pr}}^{P}$. That is, there is a proper covariant correspondence $\left(\mathcal{F}^{*}, \widetilde{V}\right):\left(B, \mathcal{G}, \mathcal{J}_{B}\right) \rightarrow(A, \mathcal{E}, \mathcal{J})$ with (invertible) 2-arrows $w:\left(\mathcal{F} \otimes_{B} \mathcal{F}^{*}, V \bullet \widetilde{V}\right) \Rightarrow$ $\left(A, \iota_{\mathcal{E}}\right)$ and $\widetilde{w}:\left(\mathcal{F}^{*} \otimes_{A} \mathcal{F}, \widetilde{V} \bullet V\right) \Rightarrow\left(B, \iota_{\mathcal{G}}\right)$.

Let $\mathcal{F}^{*}$ be the Hilbert $B, A$-bimodule adjoint to $\mathcal{F}$. For each $p \in P$, we use the identifications $\mathcal{F} \otimes_{B} \mathcal{F}^{*} \cong A$ and $\mathcal{F}^{*} \otimes_{A} \mathcal{F} \cong B$ to define a correspondence isomorphism $\widetilde{V}_{p}: \mathcal{G}_{p} \otimes_{B} \mathcal{F}^{*} \cong \mathcal{F}^{*} \otimes_{A} \mathcal{E}_{p}$ as the composite

$$
\begin{array}{rlr}
\mathcal{G}_{p} \otimes_{B} \mathcal{F}^{*} & \cong \mathcal{F}^{*} \otimes_{A} \mathcal{F} \otimes_{B} \mathcal{G}_{p} \otimes_{B} \mathcal{F}^{*} & \left(1_{\mathcal{F}} \otimes V_{p}^{-1} \otimes 1_{\mathcal{F}^{*}}\right) \\
& \cong \mathcal{F}^{*} \otimes_{A} \mathcal{E}_{p} \otimes_{A} \mathcal{F} \otimes_{B} \mathcal{F}^{*} \\
& \cong \mathcal{F}^{*} \otimes_{A} \mathcal{E}_{p}
\end{array}
$$

We set $\widetilde{V}=\left\{\widetilde{V}_{p}\right\}_{p \in P}$. Observe that $J_{p}^{A} \mathcal{F}=\mathcal{F} J_{p}^{B}$ implies $J_{p}^{B} \mathcal{F}^{*}=\mathcal{F}^{*} J_{p}^{A}$. So in order to conclude that $\left(\mathcal{F}^{*}, \widetilde{V}\right)$ is a covariant correspondence from $\left(B, \mathcal{G}, \mathcal{J}_{B}\right)$ to $(A, \mathcal{E}, \mathcal{J})$, all we need to prove is that it satisfies the coherence axiom 4.3.2. To do so, let $p, q \in P$. Since $V_{q}^{-1}$ intertwines the left actions of $A$, the following diagram commutes:

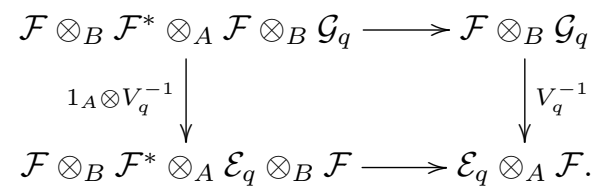

This yields the commutative diagram

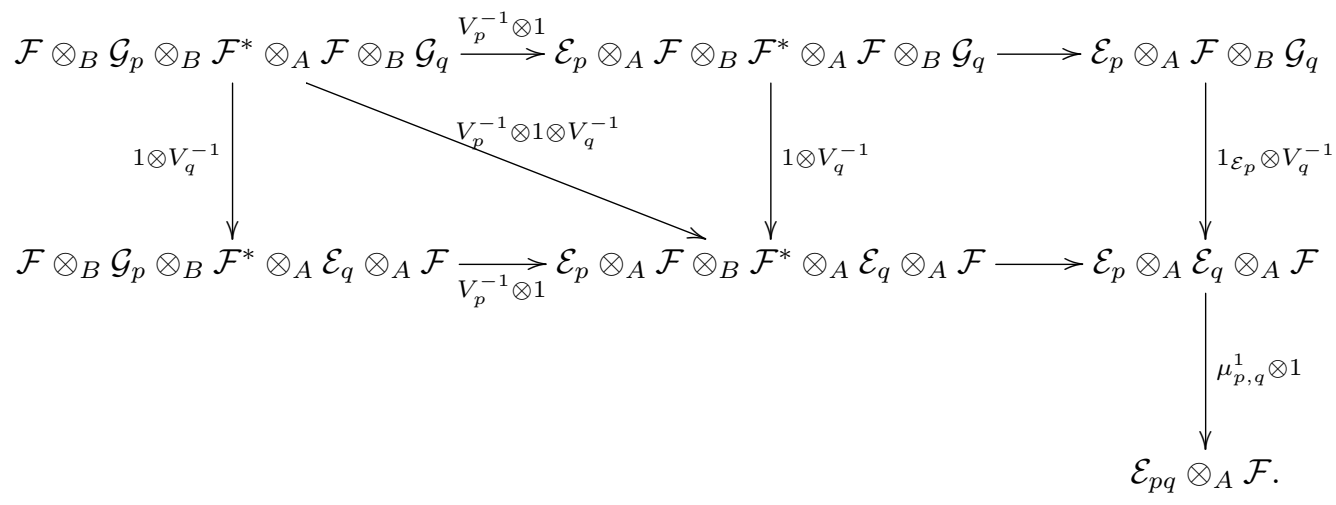

Applying the coherence axiom 4.3 .2 to $(\mathcal{F}, V)$, we deduce that the top-right composite of $(5.4 .6)$ is precisely the isomorphism

$$
\mathcal{F} \otimes_{B} \mathcal{G}_{p} \otimes_{B} \mathcal{F}^{*} \otimes_{A} \mathcal{F} \otimes_{B} \mathcal{G}_{q} \cong \mathcal{F} \otimes_{B} \mathcal{G}_{p} \otimes_{B} \mathcal{G}_{q} \stackrel{1 \otimes \mu_{p, q}^{2}}{\Longrightarrow} \mathcal{F} \otimes_{B} \mathcal{G}_{p q} \stackrel{1 \otimes V_{p q}^{-1}}{\Longrightarrow} \mathcal{E}_{p q} \otimes_{A} \mathcal{F}
$$

This corresponds to the top composite of the diagram

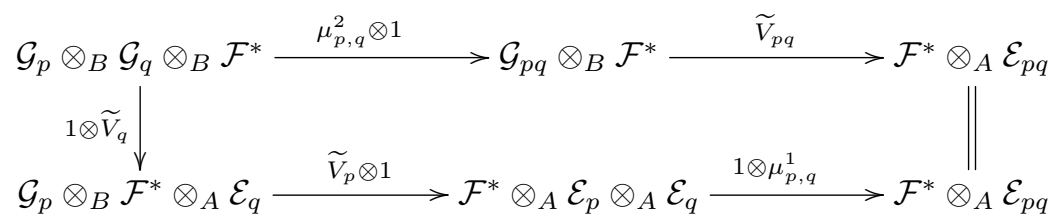

after tensoring with $1_{\mathcal{F}^{*}}$ on the left and on the right. The left-bottom composite of this diagram is obtained from that of 5.4 .6 in the same way. Hence the commutativity of (5.4.6) implies that $\left(\mathcal{F}^{*}, \widetilde{V}\right)$ is a proper covariant correspondence from $\left(B, \mathcal{G}, \mathcal{J}_{B}\right)$ to $(A, \mathcal{E}, \mathcal{J})$, as desired. The canonical isomorphisms $w: \mathcal{F} \otimes_{B} \mathcal{F}^{*} \cong A$ and $\widetilde{w}: \mathcal{F}^{*} \otimes_{A} \mathcal{F} \cong B$ are the required 2 -arrows. 


\section{FELL BUNDLES OVER QUASI-LATTICE ORDERED GROUPS}

If $(\mathcal{F}, V):\left(A, \mathcal{E}, \mathcal{I}_{\mathcal{E}}\right) \rightarrow\left(B, \mathcal{G}, \mathcal{I}_{\mathcal{G}}\right)$ is an equivalence in $\mathfrak{C}_{\mathrm{pr}, *}^{P}$ and $\left(\hat{\mathcal{G}}_{g}\right)_{g \in G}$ is amenable, then $\left(\hat{\mathcal{E}}_{g}\right)_{g \in G}$ is also amenable. Indeed, by functoriality, $\mathcal{O}_{\mathcal{F}, V}: \mathcal{O}_{\mathcal{I}_{\mathcal{E}}, \mathcal{E}} \leadsto \mathcal{O}_{\mathcal{I}_{\mathcal{G}}, \mathcal{G}}$ is an imprimitivity bimodule. In particular, $\mathcal{O}_{\mathcal{I}_{\mathcal{E}}, \mathcal{E}} \cong \mathrm{C}^{*}\left(\left(\hat{\mathcal{E}}_{g}\right)_{g \in G}\right)$ acts faithfully on $\mathcal{O}_{\mathcal{F}, V}$. Since $\mathrm{C}^{*}\left(\left(\hat{\mathcal{G}}_{g}\right)_{g \in G}\right) \cong \mathrm{C}_{r}^{*}\left(\left(\hat{\mathcal{G}}_{g}\right)_{g \in G}\right)$ through the regular representation, $\mathcal{F}^{*} \otimes_{A} \mathrm{C}_{r}^{*}\left(\left(\hat{\mathcal{E}}_{g}\right)_{g \in G}\right)$ is a faithful proper correspondence $\mathrm{C}^{*}\left(\left(\hat{\mathcal{G}}_{g}\right)_{g \in G}\right) \rightarrow$ $\mathrm{C}_{r}^{*}\left(\left(\hat{\mathcal{E}}_{g}\right)_{g \in G}\right)$ because the conditional expectation from $\mathrm{C}^{*}\left(\left(\hat{\mathcal{G}}_{g}\right)_{g \in G}\right)$ onto $B$ is faithful and the continuous projection from $\mathcal{F}^{*} \otimes_{A} \mathrm{C}_{r}^{*}\left(\left(\hat{\mathcal{E}}_{g}\right)_{g \in G}\right)$ onto $\mathcal{F}^{*} \otimes_{A} A \cong \mathcal{F}^{*}$ provides the image of $\mathrm{C}^{*}\left(\left(\hat{\mathcal{G}}_{g}\right)_{g \in G}\right)$ in $\mathbb{B}\left(\mathcal{F}^{*} \otimes_{A} \mathrm{C}_{r}^{*}\left(\left(\hat{\mathcal{E}}_{g}\right)_{g \in G}\right)\right)$ with a topological $G$-grading. From this we obtain a faithful and proper correspondence

$$
\mathcal{O}_{\mathcal{F}, V} \otimes_{\mathrm{C}^{*}\left(\left(\hat{\mathcal{G}}_{g}\right)_{g \in G}\right)} \mathcal{F}^{*} \otimes_{A} \mathrm{C}_{r}^{*}\left(\left(\hat{\mathcal{E}}_{g}\right)_{g \in G}\right): \mathrm{C}^{*}\left(\left(\hat{\mathcal{E}}_{g}\right)_{g \in G}\right) \leadsto \mathrm{C}_{r}^{*}\left(\left(\hat{\mathcal{E}}_{g}\right)_{g \in G}\right)
$$

Hence the isomorphism

$$
\left.\mathcal{O}_{\mathcal{F}, V} \otimes_{\mathrm{C}^{*}\left(\left(\hat{\mathcal{G}}_{g}\right)_{g \in G}\right)} \mathcal{F}^{*} \otimes_{A} \mathrm{C}_{r}^{*}\left(\left(\hat{\mathcal{E}}_{g}\right)_{g \in G}\right) \cong \mathcal{F} \otimes_{B} \mathcal{F}^{*} \otimes_{A} \mathrm{C}_{r}^{*}\left(\left(\hat{\mathcal{E}}_{g}\right)_{g \in G}\right) \cong \mathrm{C}_{r}^{*}\left(\hat{\mathcal{E}}_{g}\right)_{g \in G}\right)
$$

yields an injective *-homomorphism $\mathrm{C}^{*}\left(\left(\hat{\mathcal{E}}_{g}\right)_{g \in G}\right) \rightarrow \mathrm{C}_{r}^{*}\left(\left(\hat{\mathcal{E}}_{g}\right)_{g \in G}\right)$ when composed with the *-homomorphism

$$
\mathrm{C}^{*}\left(\left(\hat{\mathcal{E}}_{g}\right)_{g \in G}\right) \rightarrow \mathbb{B}\left(\mathcal{O}_{\mathcal{F}, V} \otimes_{\mathrm{C}^{*}\left(\left(\hat{\mathcal{G}}_{g}\right)_{g \in G}\right)} \mathcal{F}^{*} \otimes_{A} \mathrm{C}_{r}^{*}\left(\left(\hat{\mathcal{E}}_{g}\right)_{g \in G}\right)\right)
$$

But such a ${ }^{*}$-homomorphism coincides with the regular representation, since $\left(\mathcal{F}^{*}, \widetilde{V}\right) \circ(\mathcal{F}, V) \cong(A, \iota \mathcal{E})$. Therefore, $\left(\hat{\mathcal{E}}_{g}\right)_{g \in G}$ is amenable.

Remark 5.4.7. The fact that an equivalence between objects in $\mathfrak{C}_{\mathrm{pr}, *}^{P}$ preserves amenability could also be derived from [2] and Theorem 5.2.11.

Example 5.4.8. Let $A$ and $B$ be $\mathrm{C}^{*}$-algebras and let $\mathcal{F}: A \rightarrow B$ be an imprimitivity $A, B$-bimodule. A compactly aligned product system $\mathcal{E}=\left(\mathcal{E}_{p}\right)_{p \in P}$ over $A$ induces a compactly aligned product system $\mathcal{G}=\left(\mathcal{G}_{p}\right)_{p \in P}$ over $B$ as follows. We set $\mathcal{G}_{p}:=\mathcal{F}^{*} \otimes_{A} \mathcal{E}_{p} \otimes_{A} \mathcal{F}$. The multiplication map $\widetilde{\mu}_{p, q}: \mathcal{G}_{p} \otimes_{A} \mathcal{G}_{q} \cong$ $\mathcal{G}_{p q}$ is defined using the isomorphism $\mathcal{F} \otimes_{A} \mathcal{F}^{*} \cong A$. More explicitly, it is given by

$$
\begin{array}{rlr}
\mathcal{G}_{p} \otimes_{B} \mathcal{G}_{q} & =\mathcal{F}^{*} \otimes_{A} \mathcal{E}_{p} \otimes_{A} \mathcal{F} \otimes_{B} \mathcal{F}^{*} \otimes_{A} \mathcal{E}_{q} \otimes_{A} \mathcal{F} & \\
& \cong \mathcal{F}^{*} \otimes_{A} \mathcal{E}_{p} \otimes_{A} \mathcal{E}_{q} \otimes_{A} \mathcal{F} & \left(1_{\mathcal{F}} \otimes \mu_{p, q} \otimes 1_{\mathcal{F}}\right) \\
& \cong \mathcal{F}^{*} \otimes_{A} \mathcal{E}_{p q} \otimes_{A} \mathcal{F}=\mathcal{G}_{p q} &
\end{array}
$$

The multiplication maps $\left\{\widetilde{\mu}_{p, q}\right\}_{p, q \in P}$ satisfy the coherence axiom required for product systems because $\left\{\mu_{p, q}\right\}_{p, q \in P}$ do so.

We claim that $\mathcal{G}$ is compactly aligned. Indeed, let $p, q \in P$ with $p \vee q<\infty$. Notice that $\mathbb{K}\left(\mathcal{G}_{p}\right)$ is canonically isomorphic to $\mathcal{F}^{*} \otimes_{A} \mathbb{K}\left(\mathcal{E}_{p}\right) \otimes_{A} \mathcal{F}$ through the identification

$$
\begin{aligned}
\mathcal{F}^{*} \otimes_{A} \mathcal{E}_{p} \otimes_{A} \mathcal{F} \otimes_{B}\left(\mathcal{F}^{*} \otimes_{A} \mathcal{E}_{p} \otimes_{A} \mathcal{F}\right)^{*} & \cong \mathcal{F}^{*} \otimes_{A} \mathcal{E}_{p} \otimes_{A} \mathcal{F} \otimes_{B} \mathcal{F}^{*} \otimes_{A} \mathcal{E}_{p}^{*} \otimes_{A} \mathcal{F} \\
& \cong \mathcal{F}^{*} \otimes_{A} \mathcal{E}_{p} \otimes_{A} \mathcal{E}_{p}^{*} \otimes_{A} \mathcal{F} \\
& \cong \mathcal{F}^{*} \otimes_{A} \mathbb{K}\left(\mathcal{E}_{p}\right) \otimes_{A} \mathcal{F}
\end{aligned}
$$

So take $T \in \mathbb{K}\left(\mathcal{E}_{p}\right)$ and $S \in \mathbb{K}\left(\mathcal{E}_{q}\right)$. Let $\zeta_{1}, \zeta_{2}, \eta_{1}, \eta_{2} \in \mathcal{F}$ and let $\eta^{*} \otimes \xi \otimes \zeta$ be an elementary tensor of $\mathcal{F}^{*} \otimes_{A} \mathcal{E}_{p \vee q} \otimes_{A} \mathcal{F}$. We have that

$$
\iota_{q}^{p \vee q}\left(\eta_{2}^{*} \otimes S \otimes \zeta_{2}\right)\left(\eta^{*} \otimes \xi \otimes \zeta\right)=\eta_{2}^{*} \otimes \iota_{q}^{p \vee q}(S)\left(\varphi_{p \vee q}\left(\left\langle\left\langle\zeta_{2} \mid \eta\right\rangle\right\rangle\right)(\xi)\right) \otimes \zeta .
$$

Applying $\iota_{p}^{p \vee q}\left(\eta_{1}^{*} \otimes T \otimes \zeta_{1}\right)$ to both sides of the above equality, we deduce that

$$
\begin{aligned}
\iota_{p}^{p \vee q}\left(\eta_{1}^{*} \otimes T \otimes \zeta_{1}\right) \iota_{q}^{p \vee q}\left(\eta_{2}^{*} \otimes S \otimes \zeta_{2}\right)\left(\eta^{*} \otimes \xi \otimes \zeta\right) \\
\quad=\eta_{1}^{*} \otimes \iota_{p}^{p \vee q}(T)\left(\varphi_{p \vee q}\left(\left\langle\left\langle\zeta_{1} \mid \eta_{2}\right\rangle\right\rangle\right) \iota_{q}^{p \vee q}(S)\left(\varphi_{p \vee q}\left(\left\langle\left\langle\zeta_{2} \mid \eta\right\rangle\right\rangle\right)(\xi)\right)\right) \otimes \zeta .
\end{aligned}
$$

Define $T^{\prime} \in \mathbb{K}\left(\mathcal{E}_{p \vee q}\right)$ by $T^{\prime}=\iota_{p}^{p \vee q}(T) \varphi_{p \vee q}\left(\left\langle\left\langle\zeta_{1} \mid \eta_{2}\right\rangle\right\rangle\right) \iota_{q}^{p \vee q}(S)$. Then

$$
\eta_{1}^{*} \otimes T^{\prime}\left(\varphi_{p \vee q}\left(\left\langle\left\langle\zeta_{2} \mid \eta\right\rangle\right\rangle\right)(\xi)\right) \otimes \zeta=\left(\eta_{1}^{*} \otimes T^{\prime} \otimes \zeta_{2}\right)\left(\eta^{*} \otimes \xi \otimes \zeta\right) .
$$

So $\mathcal{G}$ is also compactly aligned, as claimed. 
Given $p \in P$, an element $b \in B$ is compact on $\mathcal{G}_{p}$ if and only if $b \mathcal{F}^{*} \subseteq \mathcal{F}^{*} \varphi_{p}^{-1}\left(\mathbb{K}\left(\mathcal{E}_{p}\right)\right)$, provided $\mathcal{F}^{*}$ is an equivalence. The bijection between the lattices of ideals of $A$ and $B$, respectively, obtained from the Rieffel correspondence, yields a one-to-one correspondence between ideals in $A$ acting by compact operators on $\mathcal{E}_{p}$ and ideals in $B$ mapped to compact operators on $\mathcal{G}_{p}$. Precisely, this sends $J_{p}^{A} \triangleleft \varphi_{p}^{-1}\left(\mathbb{K}\left(\mathcal{E}_{p}\right)\right)$ to $J_{p}^{B}=\left\langle J_{p}^{A} \mathcal{F} \mid \mathcal{F}\right\rangle$. Its inverse maps an ideal $J_{p}^{B} \triangleleft \widetilde{\varphi}_{p}^{-1}\left(\mathbb{K}\left(\mathcal{G}_{p}\right)\right)$ to $J_{p}^{A}=\left\langle\left\langle\mathcal{F} J_{p}^{B} \mid \mathcal{F}\right\rangle\right\rangle$.

The equivalence $\mathcal{F}$ may be turned into a proper covariant correspondence $(\mathcal{F}, V):\left(A, \mathcal{E}, \mathcal{J}_{A}\right) \rightarrow$ $\left(B, \mathcal{G}, \mathcal{J}_{B}\right)$, where $V=\left\{V_{p}\right\}_{p \in P}$ and $V_{p}: \mathcal{E}_{p} \otimes_{A} \mathcal{F} \cong \mathcal{F} \otimes_{B} \mathcal{G}_{p}$ arises from the canonical isomorphism

$$
\mathcal{E}_{p} \otimes_{A} \mathcal{F} \cong \mathcal{F} \otimes_{B} \mathcal{F}^{*} \otimes_{A} \mathcal{E}_{p} \otimes_{A} \mathcal{F}=\mathcal{F} \otimes_{B} \mathcal{G}_{p}
$$

Here $\mathcal{J}_{A}$ and $\mathcal{J}_{B}$ are related by the bijection described above.

It follows from Corollary 5.4.5 that $(\mathcal{F}, V)$ is invertible in $\mathfrak{C}_{\mathrm{pr}}^{P}$ and produces a Morita equivalence between $\mathcal{O}_{\mathcal{J}_{A}, \mathcal{E}}$ and $\mathcal{O}_{\mathcal{J}_{B}, \mathcal{G}}$. Therefore, up to equivariant Morita equivalence, the relative CuntzPimsner algebras associated to $\mathcal{E}$ correspond bijectively to those associated to $\mathcal{G}$. In particular, if $\mathcal{E}$ is a simplifiable product system of Hilbert bimodules, the cross sectional $\mathrm{C}^{*}$-algebra of the Fell bundle associated to $\mathcal{E}$ is Morita equivalent to that of $\mathcal{G}$. This is so because the family of Katsura's ideals $\mathcal{I}_{\mathcal{E}}$ corresponds to $\mathcal{I}_{\mathcal{G}}$ under the Rieffel correspondence.

The next proposition characterises equivalences between product systems built out of semigroups of injective endomorphisms with hereditary range as in Example 4.2.10 This generalises [42, Proposition 2.4]. The idea of the proof is also taken from there.

Proposition 5.4.9. Let $\alpha: P \rightarrow \operatorname{End}(A)$ and $\beta: P \rightarrow \operatorname{End}(B)$ be actions by extendible injective endomorphisms with hereditary range. Let ${ }_{\alpha} A$ and ${ }_{\beta} B$ be the associated product systems of Hilbert bimodules over $P^{\mathrm{op}}$. There is an equivalence $(\mathcal{F}, V):\left(A,{ }_{\alpha} A, \mathcal{I}_{\alpha} A\right) \rightarrow\left(B,{ }_{\beta} B, \mathcal{I}_{\beta} B\right)$ if and only if there are an imprimitivity $A, B$-bimodule $\mathcal{F}$ and a semigroup homomorphism $p \mapsto U_{p}$ from $P$ to the semigroup of $\mathbb{C}$-linear isometries on $\mathcal{F}$ such that, for all $p \in P$ and $\xi, \eta \in \mathcal{F}$,

$$
\left\langle\left\langle U_{p}(\xi) \mid U_{p}(\eta)\right\rangle\right\rangle=\alpha_{p}(\langle\langle\xi \mid \eta\rangle\rangle), \quad\left\langle U_{p}(\xi) \mid U_{p}(\eta)\right\rangle=\beta_{p}(\langle\xi \mid \eta\rangle) .
$$

Proof. Let $(F, V):\left(A,{ }_{\alpha} A, \mathcal{I}_{\alpha} A\right) \rightarrow\left(B,{ }_{\beta} B, \mathcal{I}_{\beta} B\right)$ be an equivalence. Then $\mathcal{F}$ is an imprimitivity $A, B$-bimodule. Observing that

$$
\left.\left\langle\left.{ }_{\beta_{p}} B\right|_{\beta_{p}} B\right\rangle\right\rangle=\beta_{p}^{-1}\left(\beta_{p}(B)\right)=B
$$

for all $p \in P$, we define a correspondence isomorphism $U_{p}^{\prime}: \mathcal{F} \rightarrow{ }_{\alpha_{p}} A \otimes_{A} \mathcal{F} \otimes_{B}{ }_{\beta_{p}} B^{*}$ by

$$
\mathcal{F} \cong \mathcal{F} \otimes_{B} \beta_{p} B \otimes_{B} \beta_{p} B^{*} \stackrel{V_{p}^{-1} \otimes 1}{\Longrightarrow}{ }_{\alpha_{p}} A \otimes_{A} \mathcal{F} \otimes_{B}{ }_{\beta_{p}} B^{*}
$$

We identify ${ }_{\beta_{p}} B^{*}$ with $B_{\beta_{p}}=B \beta_{p}(1)$ by $\widetilde{\beta_{p}(1) b} \mapsto b^{*} \beta_{p}(1)$ to obtain a linear map

$$
{ }_{\alpha_{p}} A \otimes_{A} \mathcal{F} \otimes_{B}{ }_{\beta_{p}} B^{*} \rightarrow \mathcal{F}
$$

defined on an elementary tensor $\alpha_{p}(1) a \otimes_{A} \xi \otimes_{B} b \beta_{p}(1)$ by $\left(\alpha_{p}(1) a\right) \xi\left(b \beta_{p}(1)\right)$. This is isometric because $\beta_{p}^{-1}$ is an injective ${ }^{*}$-homomorphism between $\mathrm{C}^{*}$-algebras. Its composition with $U_{p}^{\prime}$ yields a linear map $\mathcal{F} \rightarrow \mathcal{F}$, which we denote by $U_{p}$. Given $\xi, \eta \in \mathcal{F}$, we have that $\langle\xi \mid \eta\rangle=\left\langle U_{p}^{\prime}(\xi) \mid U_{p}^{\prime}(\eta)\right\rangle$, that is, $U_{p}^{\prime}$ preserves inner products. From this we deduce

$$
\left\langle U_{p}(\xi) \mid U_{p}(\eta)\right\rangle=\beta_{p}\left(\left\langle U_{p}^{\prime}(\xi) \mid U_{p}^{\prime}(\eta)\right\rangle\right)=\beta_{p}(\langle\xi \mid \eta\rangle) .
$$

Similarly, $\left\langle\left\langle U_{p}^{\prime}(\xi) \mid U_{p}^{\prime}(\eta)\right\rangle\right\rangle=\langle\langle\xi \mid \eta\rangle\rangle$ and we see that $\left\langle\left\langle U_{p}(\xi) \mid U_{p}(\eta)\right\rangle\right\rangle=\alpha_{p}(\langle\langle\xi \mid \eta\rangle\rangle)$.

It remains to verify that $p \mapsto U_{p}$ is a semigroup homomorphism from $P$ to the semigroup of $\mathbb{C}$-linear isometries on $\mathcal{F}$. First, let $\alpha_{q}(1) a \in{ }_{\alpha_{q}} A$ and notice that, given an elementary tensor $\xi \otimes \alpha_{p}(1) b$ of $\mathcal{F} \otimes_{B} \beta_{p} B$, one has

$$
V_{p}^{-1}\left(\alpha_{q}(1) a \xi \otimes \beta_{p}(1) b\right)=\alpha_{q}(1) a V_{p}^{-1}\left(\xi \otimes \beta_{p}(1) b\right)
$$

Since the left action of $A$ on ${ }_{\alpha_{p}} A$ is implemented by $\alpha_{p}$, it follows that the image of $V_{p}^{-1}\left(\alpha_{q}(1) a \xi \otimes \beta_{p}(1) b\right)$ in $\mathcal{F}$ under the map ${ }_{\alpha_{p}} A \otimes_{A} \mathcal{F} \rightarrow \mathcal{F}$ determined by the left action of $A$ on $\mathcal{F}$ coincides with the image 


\section{FELL BUNDLES OVER QUASI-LATTICE ORDERED GROUPS}

of

$$
\left(\mu_{q, p}^{\alpha} \otimes 1\right)\left(\alpha_{q}(1) a \otimes_{A} V_{p}^{-1}\left(\xi \otimes \beta_{p}(1) b\right)\right)
$$

under the corresponding map $\alpha_{p q} A \otimes_{A} \mathcal{F} \rightarrow \mathcal{F}$. Here $\mu_{q, p}^{\alpha}$ is the correspondence isomorphism $\alpha_{q} A \otimes_{A}$ $\alpha_{p} A \cong{ }_{\alpha_{p q}} A$.

Now let $p, q \in P$ and let $\left(u_{\lambda}\right)_{\lambda \in \Lambda}$ be an approximate identity for $B$. Fix $\lambda \in \Lambda$ and let $\xi \in \mathcal{F}$ and $b \in B$. Then

$$
U_{q}^{\prime}\left(\xi u_{\lambda} u_{\lambda} b\right)=V_{q}^{-1}\left(\xi \otimes \beta_{q}\left(u_{\lambda}\right)\right) \otimes \beta_{q}\left(u_{\lambda} b\right) .
$$

From the above observation and from the fact that $V_{p}^{-1}$ and $V_{q}^{-1}$ intertwine the right actions of $B$, we conclude that

$$
U_{p}^{\prime} U_{q}\left(\xi u_{\lambda} u_{\lambda} b\right)=\left(\mu_{q, p}^{\alpha} \otimes 1\right)\left(1 \otimes V_{p}^{-1} \otimes 1_{B_{\beta_{p}}}\right)\left(V_{q}^{-1}\left(\xi \otimes \beta_{q}\left(u_{\lambda}\right)\right) \otimes \beta_{p q}\left(u_{\lambda}\right) \otimes \beta_{p q}(b)\right) .
$$

Combining this with the coherence condition 4.3 .2 we may replace the right-hand side of the above equality by

$$
\begin{array}{r}
\left.\left(V_{p q}^{-1} \otimes 1_{B_{\beta_{p}}}\right)\left(1 \otimes \mu_{q, p}^{\beta} \otimes 1\right)\right)\left(\xi \otimes\left(\beta_{q}\left(u_{\lambda}\right) \otimes \beta_{p q}\left(u_{\lambda}\right)\right) \otimes \beta_{p q}(b)\right)= \\
=\left(V_{p q}^{-1} \otimes 1_{B_{\beta_{p}}}\right)\left(\xi \otimes \beta_{p q}\left(u_{\lambda} u_{\lambda}\right)\right) \otimes \beta_{p q}(b) .
\end{array}
$$

This implies $U_{p} U_{q}\left(\xi u_{\lambda} u_{\lambda} b\right)=U_{p q}\left(\xi u_{\lambda} u_{\lambda} b\right)$. Using that all the $U_{p}$ 's are continuous and

$$
\xi b=\lim _{\lambda}\left(\xi u_{\lambda} u_{\lambda} b\right),
$$

we obtain $U_{p} U_{q}(\xi b)=U_{p q}(\xi b)$. This shows that $p \mapsto U_{p}$ is a semigroup homomorphism, as asserted.

Conversely, suppose that we are given an imprimitivity $A, B$-bimodule $\mathcal{F}$ and a semigroup homomorphism $p \mapsto U_{p}$ from $P$ to the semigroup of $\mathbb{C}$-linear isometries on $\mathcal{F}$ satisfying (5.4.10). For each $p \in P, \xi \in \mathcal{F}$ and $b \in B$, we have that $U_{p}(\xi b)=U_{p}(\xi) \beta_{p}(b)$ because

$$
\begin{aligned}
\left\langle U_{p}(\xi b)-U_{p}(\xi) \beta_{p}(b) \mid U_{p}(\xi b)-U_{p}(\xi) \beta_{p}(b)\right\rangle & =\left\langle U_{p}(\xi b) \mid U_{p}(\xi b)\right\rangle-\left\langle U_{p}(\xi b) \mid U_{p}(\xi) \beta_{p}(b)\right\rangle \\
-\left\langle U_{p}(\xi) \beta_{p}(b) \mid U_{p}(\xi b)\right\rangle & +\left\langle U_{p}(\xi) \beta_{p}(b) \mid U_{p}(\xi) \beta_{p}(b)\right\rangle \\
& =\beta_{p}(\langle\xi b \mid \xi b\rangle)-\beta_{p}(\langle\xi b \mid \xi\rangle) \beta_{p}(b) \\
-\beta_{p}(b)^{*} \beta_{p}(\langle\xi \mid \xi b\rangle) & +\beta_{p}\left(b^{*}\right) \beta_{p}(\langle\xi \mid \xi\rangle) \beta_{p}(b)=0 .
\end{aligned}
$$

The same reasoning shows that $U_{p}(a \xi)=\alpha_{p}(a) U_{p}(\xi)$ for all $a \in A$.

We then define a map $V_{p}^{\prime}:{ }_{\alpha_{p}} A^{*} \otimes_{A} \mathcal{F} \otimes_{B} \beta_{p} B \rightarrow \mathcal{F}$ on elementary tensors by

$$
a \alpha_{p}(1) \otimes \xi \otimes_{B} \beta_{p}(1) b \mapsto a U_{p}(\xi) b .
$$

In order to verify that this preserves the $B$-valued inner product, let $a, c \in A, b, d \in B$ and $\xi, \eta \in \mathcal{F}$. Let $\left(u_{\lambda}\right)_{\lambda \in \Lambda}$ be an approximate identity for $A$ and fix $\lambda \in \Lambda$. Then

$$
\begin{aligned}
\left\langle a U_{p}\left(u_{\lambda} \xi\right) b \mid c U_{p}\left(u_{\lambda} \eta\right) d\right\rangle & =b^{*}\left\langle a \alpha_{p}\left(u_{\lambda}\right) U_{p}(\xi) \mid c \alpha_{p}\left(u_{\lambda}\right) U_{p}(\eta)\right\rangle d \\
& =b^{*}\left\langle U_{p}(\xi) \mid \alpha_{p}\left(u_{\lambda}\right) a^{*} c \alpha_{p}\left(u_{\lambda}\right) U_{p}(\eta)\right\rangle d \\
& =b^{*}\left\langle U_{p}(\xi) \mid U_{p}\left(\alpha_{p}^{-1}\left(\alpha_{p}\left(u_{\lambda}\right) a^{*} c \alpha_{p}\left(u_{\lambda}\right)\right) \eta\right)\right\rangle d \\
& =b^{*} \beta_{p}\left(\left\langle\xi \mid \alpha_{p}^{-1}\left(\alpha_{p}\left(u_{\lambda}\right) a^{*} c \alpha_{p}\left(u_{\lambda}\right)\right) \eta\right\rangle\right) d \\
& =\left\langle a \alpha_{p}\left(u_{\lambda}\right) \otimes \xi \otimes \beta_{p}(1) b \mid c \alpha_{p}\left(u_{\lambda}\right) \otimes \eta \otimes \beta_{p}(1) d\right\rangle .
\end{aligned}
$$

Using that $U_{p}$ is continuous and $\xi=\lim _{\lambda} u_{\lambda} \xi, \eta=\lim _{\lambda} u_{\lambda} \eta$, we conclude that $V_{p}^{\prime}$ preserves the inner product. In addition, it intertwines the left and right actions of $A$ and $B$.

Now we let $\widetilde{V}_{p}: \mathcal{F} \otimes_{B}{ }_{\beta_{p}} B \Rightarrow{ }_{\alpha_{p}} A \otimes_{A} \mathcal{F}$ be the composite

$$
\mathcal{F} \otimes_{B} \beta_{p} B \cong{ }_{\alpha_{p}} A \otimes_{A} \alpha_{p} A^{*} \otimes_{A} \mathcal{F} \otimes_{B} \beta_{p} B \stackrel{1 \otimes V_{p}^{\prime}}{\Longrightarrow} \alpha_{p} A \otimes_{A} \mathcal{F},
$$

where the isomorphism on the left-hand side comes from the identification

$$
{ }_{\alpha_{p}} A \otimes_{A} \alpha_{p} A^{*} \cong\left\langle\left\langle{ }_{\alpha_{p}} A \mid{ }_{\alpha_{p}} A\right\rangle\right\rangle=A .
$$


Then $\widetilde{V}_{p}$ is an isometry between correspondences $A \leadsto B$. To see that it is indeed unitary, we need to prove that it is also surjective.

First, observe that

$$
\alpha_{p}(\langle\langle\xi \mid \eta\rangle\rangle) \zeta=\left\langle\left\langle U_{p}(\xi) \mid U_{p}(\eta)\right\rangle\right\rangle \zeta=U_{p}(\xi)\left\langle U_{p}(\eta) \mid \zeta\right\rangle
$$

This implies $\alpha_{p}(A) \mathcal{F}=U_{p}(\mathcal{F})\left\langle U_{p}(\mathcal{F}) \mid \mathcal{F}\right\rangle$, provided $\langle\langle\mathcal{F} \mid \mathcal{F}\rangle\rangle=A$. Again we let $\left(u_{\lambda}\right)_{\lambda \in \Lambda}$ be an approximate identity for $A$ and fix $\lambda \in \Lambda$. Let $c \in A$ be such that $u_{\lambda}=c^{*} c$. Take $a \in A$ and $\xi \in \mathcal{F}$. Then

$$
\alpha_{p}\left(u_{\lambda}\right) a \otimes_{A} \xi=\alpha_{p}\left(c^{*}\right) \otimes_{A} \alpha_{p}(c)(a \xi) \in \alpha_{p}\left(c^{*}\right) \otimes_{A} U_{p}(\mathcal{F})\left\langle U_{p}(\mathcal{F}) \mid \mathcal{F}\right\rangle .
$$

Using that $U_{p}(\mathcal{F})=\alpha_{p}(A) U_{p}(\mathcal{F})$, we deduce that $\alpha_{p}\left(u_{\lambda}\right) a \otimes_{A} \xi$ belongs to the image of $\tilde{V}_{p}$. This has closed range and hence $\alpha_{p}(1) a \otimes \xi$ also lies in $\widetilde{V}_{p}\left(\mathcal{F} \otimes_{B} \beta_{p} B\right)$. Applying again the fact that $\widetilde{V}_{p}$ has closed range, we conclude that it is indeed unitary.

We let $V_{p}=\widetilde{V}_{p}^{*}$ and $V=\left\{V_{p}\right\}_{p \in P}$. We shall now prove that $(\mathcal{F}, V)$ is a proper covariant correspondence. In this case, it suffices to show that it satisfies the coherence axiom (4.3.2) and that $V_{e}$ is the canonical isomorphism obtained from the left and right actions of $A$ and $B$, respectively. This latter fact follows from the identities

$$
\left\langle\left\langle U_{e}(\xi) \mid \eta\right\rangle\right\rangle=\left\langle\left\langle\xi \mid U_{e}(\eta)\right\rangle\right\rangle=\langle\langle\xi \mid \eta\rangle\rangle=\left\langle\left\langle U_{e}(\xi) \mid U_{e}(\eta)\right\rangle,\right.
$$

so that $U_{e}=\operatorname{id}_{\mathcal{F}}$. The above equalities may be derived from the computation

$$
\left\langle\left\langle U_{e}(\xi) \mid \eta\right\rangle\right\rangle=\alpha_{e}\left(\left\langle\left\langle U_{e}(\xi) \mid \eta\right\rangle\right\rangle\right)=\left\langle\left\langle U_{e}\left(U_{e}(\xi)\right) \mid U_{e}(\eta)\right\rangle\right\rangle=\left\langle\left\langle U_{e}(\xi) \mid U_{e}(\eta)\right\rangle\right\rangle=\langle\langle\xi \mid \eta\rangle\rangle .
$$

Finally, given $a, c \in A, b, d \in B$ and $\xi \in \mathcal{F}$, we have

$$
c U_{q}\left(a U_{p}(\xi) b\right) d=c \alpha_{q}(a) U_{q}\left(U_{p}(\xi)\right) \beta_{q}(b) d=c \alpha_{q}(a) U_{q p}(\xi) \beta_{q}(b) d .
$$

This leads to a commmutative diagram for $\widetilde{V}_{p}, \widetilde{V}_{q}$ and $\widetilde{V}_{q p}$ as in 4.3.2. By reversing arrows, we conclude that $(\mathcal{F}, V)$ also makes such a diagram commute. This completes the proof. 


\section{Chapter 6}

\section{$\mathrm{C}^{*}$-algebras for product systems over subsemigroups of groups}

In this chapter, we treat product systems over semigroups that can be embedded in groups. We combine ideas of Exel and Sims and Yeend (see $[22,55]$ ) to construct a $\mathrm{C}^{*}$-algebra $A \times \mathcal{E} P$ out of a product system $\mathcal{E}$ so that a representation of $A \times_{\mathcal{E}} P$ is faithful on its fixed-point algebra for the canonical coaction of a group containing $P$ if and only if it is faithful on the coefficient algebra. We begin by considering a family of representations of the Toeplitz algebra of $\mathcal{E}$. We use its topological grading from Lemma 4.1 .6 to define an ideal in $\mathcal{T}_{\mathcal{E}}$, so that the coaction descends to the corresponding quotient. We then prove that $A$ embeds into this quotient. This is done in Section 6.1

In Section 6.2 we show that a representation of such a quotient of $\mathcal{T}_{\mathcal{E}}$ is faithful on its fixed-point algebra if and only if it is faithful on $A$. We apply this to prove that this construction does not depend on the choice of the group containing $P$. Then in Theorem 6.2.5 we introduce what we call the covariance algebra of $\mathcal{E}$. We finish this chapter with examples of $\mathrm{C}^{*}$-algebras that can be described as covariance algebras of product systems. We also discuss the relationship of these algebras to Cuntz-Nica-Pimsner algebras.

\subsection{Strongly covariant representations}

We first introduce the notion of strongly covariant representations. Let $P$ be a semigroup with unit $e$. Assume that $P$ is embeddable into a group. That is, there is a group $G$ and an injective semigroup homomorphism $\gamma: P \rightarrow G$. Fix a $\mathrm{C}^{*}$-algebra $A$ and a product system $\mathcal{E}=\left(\mathcal{E}_{p}\right)_{p \in P}$ over $A$.

Let $F \subseteq G$ be a finite subset. We set

$$
K_{F}:=\bigcap_{g \in F} g P
$$

So $K_{\{e, g\}} \neq \emptyset$ if and only if $g$ may be written as $p q^{-1}$ for some $p, q \in P$. In addition, $K_{g F}=g K_{F}$ for all $g \in G$, where

$$
g F=\{g h \mid h \in F\} .
$$

If $p \in P$ and $p \in K_{\{p, g\}}$, then $p=g q$ for some $q \in P$, which implies $g=p q^{-1}$.

For each $p \in P$ and each $F \subseteq G$ finite, we define an ideal $I_{p^{-1}(p \vee F)} \triangleleft A$ as follows. Given $g \in F$, we let

$$
I_{p^{-1} K_{\{p, g\}}}:= \begin{cases}\bigcap_{r \in K_{\{p, g\}}} \operatorname{ker} \varphi_{p^{-1} r} & \text { if } K_{\{p, g\}} \neq \emptyset \text { and } p \notin K_{\{p, g\}}, \\ A & \text { otherwise. }\end{cases}
$$

We then let

$$
I_{p^{-1}(p \vee F)}:=\bigcap_{g \in F} I_{p^{-1}} K_{\{p, g\}} .
$$


This gives a new correspondence $\mathcal{E}_{F}: A \leadsto A$ by setting

$$
\mathcal{E}_{F}:=\bigoplus_{p \in P} \mathcal{E}_{p} I_{p^{-1}(p \vee F)}
$$

Finally, let $\mathcal{E}_{F}^{+}$denote the right Hilbert $A$-module $\bigoplus_{g \in G} \mathcal{E}_{g F}$. For each $\xi \in \mathcal{E}_{p}$, we define an operator $t_{F}^{p}(\xi) \in \mathbb{B}\left(\mathcal{E}_{F}^{+}\right)$so that it maps the direct summand $\mathcal{E}_{g F}$ into $\mathcal{E}_{p g F}$ for all $g \in G$. Explicitly,

$$
t_{F}^{p}(\xi)\left(\eta_{r}\right):=\mu_{p, r}\left(\xi \otimes_{A} \eta_{r}\right), \quad \eta_{r} \in \mathcal{E}_{r} I_{r^{-1}(r \vee g F)} .
$$

This is well defined because $I_{r^{-1}(r \vee F)}=I_{(p r)^{-1}(p r \vee p F)}$ for each $F \subseteq G$ finite and each $p \in P$. Its adjoint $t_{F}^{p}(\xi)^{*}$ sends $\mu_{p, r}\left(\zeta_{p} \otimes \eta_{r}\right)$ to $\varphi_{r}\left(\left\langle\xi \mid \zeta_{p}\right\rangle\right) \eta_{r}$. This is well defined because $I_{s^{-1}(s \vee F)}=I_{s^{-1} p\left(p^{-1} s \vee p^{-1} F\right)}$ for all $s \in p P$. This gives a representation $t_{F}=\left\{t_{F}^{p}\right\}_{p \in P}$ of $\mathcal{E}$ and hence a ${ }^{*}$-homomorphism $\mathcal{T}_{\mathcal{E}} \rightarrow \mathbb{B}\left(\mathcal{E}_{F}^{+}\right)$, which we still denote by $t_{F}$.

Let us denote by $Q_{g}^{F}$ the projection of $\mathcal{E}_{F}^{+}$onto the direct summand $\mathcal{E}_{g F}$. Then

$$
t_{F}^{p}(\xi) Q_{g}^{F}=Q_{p g}^{F} t_{F}^{p}(\xi), \quad t_{F}^{p}(\xi)^{*} Q_{g}^{F}=Q_{p^{-1} g}^{F} t_{F}^{p}(\xi)^{*}
$$

for all $p \in P$. Set

$$
\mathcal{T}_{\mathcal{E}}^{e, F}:=Q_{e}^{F} \mathcal{T}_{\mathcal{E}}^{e} Q_{e}^{F},
$$

where $\mathcal{T}_{\mathcal{E}}^{e}$ is the fixed-point algebra of $\mathcal{T}_{\mathcal{E}}$ for the gauge coaction of $G$. If $F_{1} \subseteq F_{2}$ are finite subsets of $G$, then

$$
I_{p^{-1}\left(p \vee F_{1}\right)} \supseteq I_{p^{-1}\left(p \vee F_{2}\right)}
$$

for all $p \in P$. Hence $\mathcal{E}_{F_{2}}$ may be regarded as a closed submodule of $\mathcal{E}_{F_{1}}$. The restriction of $Q_{e}^{F_{1}} \mathcal{T}_{\mathcal{E}}^{e} Q_{e}^{F_{1}}$ to $\mathcal{E}_{F_{2}}$ gives a ${ }^{*}$-homomorphism $t_{F_{1}, F_{2}}: \mathcal{T}_{\mathcal{E}}^{e, F_{1}} \rightarrow \mathcal{T}_{\mathcal{E}}^{e, F_{2}}$ satisfying $t_{F_{1}, F_{2}} \circ t_{F_{1}}=t_{F_{2}}$ on $\mathcal{T}_{\mathcal{E}}^{e}$. For $F_{1} \subseteq F_{2} \subseteq F_{3}$, we have $t_{F_{2}, F_{3}} \circ t_{F_{1}, F_{2}}=t_{F_{1}, F_{3}}$. So we let $F$ range in the directed set determined by all finite subsets of $G$ and define an ideal $J_{e} \triangleleft \mathcal{T}_{\mathcal{E}}^{e}$ by

$$
J_{e}:=\left\{b \in \mathcal{T}_{\mathcal{E}}^{e} \mid \lim _{F}\|b\|_{F}=0\right\},
$$

where $\|b\|_{F}:=\left\|t_{F}(b)\right\|$. We are now ready to introduce our notion of covariant representations.

Definition 6.1.2. We will say that a representation of $\mathcal{E}$ is strongly covariant if it vanishes on $J_{e}$.

Let $J_{\infty} \triangleleft \mathcal{T}_{\mathcal{E}}$ be the ideal generated by $J_{e}$. Then $\mathcal{T}_{\mathcal{E}} / J_{\infty}$ is universal for strongly covariant representations of $\mathcal{E}$.

The idea behind 6.1 .1 started from the realisation that the correspondences $\widetilde{\mathcal{E}}_{p}$ 's built in 55 out of $\mathcal{E}$ could be replaced by the $\mathcal{E}_{F}$ 's in order to give the same notion of covariant representations if $\mathcal{E}$ is compactly aligned and $\widetilde{\phi}$-injective and $P$ is directed. This is shown in Proposition 6.3.6. In this case, it suffices to consider finite subsets of $P$ because $(G, P)$ is quasi-lattice ordered. Exel constructed a $\mathrm{C}^{*}$-algebra out of a nondegenerate interaction group $(A, G, V)$ with the property that a representation of this crossed product is faithful on the fixed-point algebra for the canonical coaction of $G$ if and only if it is faithful on $A$. To show that $A$ embeds into the crossed product, he built a faithful covariant representation by using inductive limits over finite subsets of $G$ (see 22, Section 9]). This is related to product systems because, in fact, the main purpose in 22 was to introduce a new notion of crossed products by semigroups of unital and injective endomorphisms which can be enriched to interaction groups. Here we want to associate a $\mathrm{C}^{*}$-algebra to a product system $\mathcal{E}=\left(\mathcal{E}_{p}\right)_{p \in P}$ with the property that a representation of this resulting $\mathrm{C}^{*}$-algebra is faithful on the fixed-point algebra for the canonical coaction of a group conaining $P$ if and only if it is faithful on $A$. To achieve this goal, we believe its unit fibre should be a direct limit of $C^{*}$-algebras with injective connecting maps (see [5, 22, 34, 48]), although in general this fact is not established. So, combining all these ideas and modifying the Cuntz-Nica-Pimsner covariance condition accordingly, we arrived at the $\mathcal{E}_{F}$ 's and Definition 6.1.2

Our next immediate goal is to prove that $A$ embeds into the quotient $\mathcal{T}_{\mathcal{E}} / J_{\infty}$. 
Lemma 6.1.3. The ideal $J_{\infty}$ coincides with $\overline{\bigoplus_{g \in G}} \mathcal{T}_{\mathcal{E}}^{g} J_{e}$. As a consequence,

$$
J_{\infty}=\bar{\bigoplus}_{g \in G}\left(J_{\infty} \cap \mathcal{T}_{\mathcal{E}}^{g}\right) .
$$

Proof. In order to prove the first assertion, it suffices to show that $J_{e} \mathcal{T}_{\mathcal{E}}^{g} \subseteq \mathcal{T}_{\mathcal{E}}^{g} J_{e}$ for all $g \in G$. To do so, let $b \in J_{e}$ and $0 \neq c_{g} \in \mathcal{T}_{\mathcal{E}}^{g}$. Let $\varepsilon>0$. There is $F \subseteq G$ finite with $\|b\|_{S}<\frac{\varepsilon}{\left\|c_{g}\right\|}$ for all finite subsets $S$ of $G$ with $S \supseteq F$ because $b \in J_{e}$. Set

$$
F^{\prime}:=g^{-1} F=\left\{g^{-1} h \mid h \in F\right\} .
$$

Since $c_{g}$ maps $\mathcal{E}_{F^{\prime}}$ into $\mathcal{E}_{g F^{\prime}}=\mathcal{E}_{F}$, it follows that $\left\|c_{g}^{*} b^{*} b c_{g}\right\|_{F^{\prime}}<\varepsilon^{2}$. This guarantees that

$$
\left(J_{e} \mathcal{T}_{\mathcal{E}}^{g}\right)^{*}\left(J_{e} \mathcal{T}_{\mathcal{E}}^{g}\right) \subseteq J_{e}
$$

By Lemma 3.1.8, $J_{e} \mathcal{T}_{\mathcal{E}}^{g} \subseteq \mathcal{T}_{\mathcal{E}}^{g} J_{e}$. Applying the first assertion and the continuity of the projection of $\mathcal{T}_{\mathcal{E}}$ onto $\mathcal{T}_{\mathcal{E}}^{g}$, we deduce that $J_{\infty} \cap \mathcal{T}_{\mathcal{E}}^{g}=\mathcal{T}_{\mathcal{E}}^{g} J_{e}$. This gives the last statement.

Lemma 6.1.4. Let $q: \mathcal{T}_{\mathcal{E}} \rightarrow \mathcal{T}_{\mathcal{E}} / J_{\infty}$ be the quotient map. There is a full coaction $\delta: \mathcal{T}_{\mathcal{E}} / J_{\infty} \rightarrow$ $\mathcal{T}_{\mathcal{E}} / J_{\infty} \otimes \mathrm{C}^{*}(G)$ satisfying $\delta \circ q=\left(q \otimes \mathrm{id}_{\mathrm{C}^{*}(G)}\right) \circ \widetilde{\delta}$. Moreover, the spectral subspace for $\delta$ at $g \in G i s$ canonically isomorphic to $\mathcal{T}_{\mathcal{E}}^{g} / \mathcal{T}_{\mathcal{E}}^{g} J_{e}$.

Proof. Given $c \in \mathcal{T}_{\mathcal{E}} / J_{\infty}$, choose $b \in \mathcal{T}_{\mathcal{E}}$ with $q(b)=c$ and set

$$
\delta(q(b)):=\left(q \otimes \operatorname{id}_{\mathrm{C}^{*}(G)}\right) \widetilde{\delta}(b) .
$$

Lemma 6.1 .3 and Proposition 2.2.12 say that this is indeed a well-defined full coaction of $G$ on $\mathcal{T}_{\mathcal{E}} / J_{\infty}$. The equality $\delta \circ q=\left(q \otimes \operatorname{id}_{\mathrm{C}^{*}(G)}\right) \circ \delta$ follows from the definition of $\delta$.

In order to prove the last assertion, let $\left(\mathcal{T}_{\mathcal{E}} / J_{\infty}\right)^{g}$ denote the spectral subspace at $g \in G$ for the coaction $\delta$. Clearly, the map which sends $b_{g} \in \mathcal{T}_{\mathcal{E}}^{g}$ to $q\left(b_{g}\right)$ vanishes on $\mathcal{T}_{\mathcal{E}}^{g} J_{e}$. Moreover, Lemma 6.1.3 implies that this produces an injective map from $\mathcal{T}_{\mathcal{E}}^{g} / \mathcal{T}_{\mathcal{E}}^{g} J_{e}$ into $\left(\mathcal{T}_{\mathcal{E}} / J_{\infty}\right)^{g}$. That it is also surjective follows by the same argument used in Lemma 4.1.6

We will often use the above description of the $G$-grading for $\mathcal{T}_{\mathcal{E}} / J_{\infty}$.

Proposition 6.1.5. The quotient map $q: \mathcal{T}_{\mathcal{E}} \rightarrow \mathcal{T}_{\mathcal{E}} / J_{\infty}$ is injective on $\tilde{t}(A)$.

Proof. We will show that $\tilde{t}(A) \cap J_{e}=0$ in $\mathcal{T}_{\mathcal{E}}$. This implies the conclusion by the previous lemma.

Let $F \subseteq G$ be finite and $0 \neq a \in A$. We claim that $t_{F}^{e}(a) \neq 0$ on $\mathcal{E}_{F}$. Indeed, if $a I_{e \vee F} \neq 0$ we are done. Otherwise, $a \notin I_{K_{\left\{e, g_{1}\right\}}}$ for some $g_{1} \in F$, because $I_{e \vee F}=\bigcap_{g \in G} I_{K_{\{e, g\}}}$. Since

$$
I_{K_{\left\{e, g_{1}\right\}}}=\bigcap_{r \in g_{1} P \cap P} \operatorname{ker} \varphi_{r}
$$

there exists $r_{1} \in P \cap g_{1} P$ with $\varphi_{r_{1}}(a) \neq 0$. Put

$$
F_{1}:=\left\{g \in F \mid K_{\left\{r_{1}, g\right\}} \neq \emptyset \text { and } r_{1} \notin K_{\left\{r_{1}, g\right\}}\right\} \text {. }
$$

Thus $g_{1} \notin F_{1}$. So $F_{1} \subsetneq F$ and $\mathcal{E}_{r_{1}} I_{r_{1}^{-1}\left(r_{1} \vee F\right)}=\mathcal{E}_{r_{1}} I_{r_{1}^{-1}\left(r_{1} \vee F_{1}\right)}$. Our claim is proved if $\varphi_{r_{1}}(a) \neq 0$ on $\mathcal{E}_{r_{1}} I_{r_{1}^{-1}\left(r_{1} \vee F_{1}\right)}$. This is so, in particular, if $F_{1}=\emptyset$ because $\varphi_{r_{1}}(a) \neq 0$ on $\mathcal{E}_{r_{1}}$. Assume that $a$ acts trivially on $\mathcal{E}_{r_{1}} I_{r_{1}^{-1}\left(r_{1} \vee F_{1}\right)}$. Then

$$
\left\langle\varphi_{r_{1}}(a) \mathcal{E}_{r_{1}} \mid \varphi_{r_{1}}(a) \mathcal{E}_{r_{1}}\right\rangle \cap I_{r_{1}^{-1}\left(r_{1} \vee F_{1}\right)}=\{0\}
$$

Thus there exist $g_{2} \in F_{1}$ and $\xi \in \mathcal{E}_{r_{1}}$ so that $\left\langle\varphi_{r_{1}}(a)(\xi) \mid \varphi_{r_{1}}(a)(\xi)\right\rangle \notin I_{r_{1}^{-1}\left(r_{1} \vee g_{2}\right)}$. As a consequence, one can find $r_{2} \in K_{\left\{r_{1}, g_{2}\right\}}$ such that

$$
\left\langle\varphi_{r_{1}}(a)(\xi) \mid \varphi_{r_{1}}(a)(\xi)\right\rangle \notin \operatorname{ker} \varphi_{r_{1}^{-1} r_{2}}
$$


We see that $a \notin \operatorname{ker} \varphi_{r_{2}}$ because $\mu_{r_{1}, r_{1}^{-1} r_{2}}: \mathcal{E}_{r_{1}} \otimes_{A} \mathcal{E}_{r_{1}^{-1} r_{2}} \rightarrow \mathcal{E}_{r_{2}}$ is an isomorphism of correspondences. Let

$$
F_{2}:=\left\{g \in F \mid K_{\left\{r_{2}, g\right\}} \neq \emptyset \text { and } r_{2} \notin K_{\left\{r_{2}, g\right\}}\right\} \text {. }
$$

Notice that $F_{2} \subsetneq F_{1}$ and

$$
\mathcal{E}_{r_{2}} I_{r_{2}^{-1}\left(r_{2} \vee F\right)}=\mathcal{E}_{r_{2}} I_{r_{2}^{-1}\left(r_{2} \vee F_{2}\right)} .
$$

If $\varphi_{r_{2}}(a)$ vanishes on $\mathcal{E}_{r_{2}} I_{r_{2}^{-1}\left(r_{2} \vee F_{2}\right)}$, then the same reasoning as above yields $g_{3} \in F_{2}$ and $r_{3} \in K_{\left\{r_{2}, g_{3}\right\}}$ with $\varphi_{r_{3}}(a) \neq 0$ on $\mathcal{E}_{r_{3}}$. Set

$$
F_{3}:=\left\{g \in F \mid K_{\left\{r_{3}, g\right\}} \neq \emptyset \text { and } r_{3} \notin K_{\left\{r_{3}, g\right\}}\right\} .
$$

We then have $F_{3} \subsetneq F_{2} \subsetneq F_{1} \subsetneq F$ and $\mathcal{E}_{r_{3}} I_{r_{3}^{-1}\left(r_{3} \vee F\right)}=\mathcal{E}_{r_{3}} I_{r_{3}^{-1}\left(r_{3} \vee F_{3}\right)}$. This process cannot continue infinitely because $F$ is finite. So we must stop at some $r_{j}$ with $\varphi_{r_{j}}(a) \neq 0$ on $\mathcal{E}_{r_{j}} I_{r_{j}^{-1}\left(r_{j} \vee F\right)}$.

Thus $t_{F}^{e}(a)$ is nonzero on $\mathcal{E}_{F}$. Therefore, for all $a \in A$, we have that

$$
\lim _{F}\|a\|_{F}=\lim _{F}\|a\|=\|a\| \text {. }
$$

This completes the proof.

\subsection{Covariance algebras associated to product systems}

Our goal in this section is to associate a $\mathrm{C}^{*}$-algebra $A \times{ }_{\mathcal{E}} P$ to a given product system $\left(\mathcal{E}_{p}\right)_{p \in P}$ satisfying two properties: the representation of $\mathcal{E}$ in $A \times{ }_{\mathcal{E}} P$ is injective and any representation of $A \times{ }_{\mathcal{E}} P$ in a $\mathrm{C}^{*}$-algebra $B$ that is faithful on $A$ is also faithful on the fixed-point algebra $\left(A \times{ }_{\mathcal{E}} P\right)^{\delta}$ for the canonical gauge coaction of $G$ on $A \times_{\mathcal{E}} P$, where $G$ is a group with $P \subseteq G$. A candidate for $A \times_{\mathcal{E}} P$ is of course the universal $\mathrm{C}^{*}$-algebra for strongly covariant representations introduced previously. We shall prove that this is independent of the choice of the group containing $P$.

Lemma 6.2.1. Let $P$ be a subsemigroup of a group $G$ and $\mathcal{E}$ a product system over $P$. Let $\psi=$ $\left\{\psi_{p}\right\}_{p \in P}$ be a strongly covariant representation of $\mathcal{E}$ in a $\mathrm{C}^{*}$-algebra $B$. The resulting ${ }^{*}$-homomorphism $\widetilde{\psi}: \mathcal{T}_{\mathcal{E}} / J_{\infty} \rightarrow B$ is faithful on $\mathcal{T}_{\mathcal{E}}^{e} / J_{e}$ if and only if $\psi_{e}$ is injective.

Proof. If $\widetilde{\psi}$ is faithful on $\mathcal{T}_{\mathcal{E}}^{e} / J_{e}$, then Proposition 6.1 .5 implies that $\psi_{e}$ is injective. Suppose that $\psi$ is strongly covariant and $\psi_{e}$ is faithful. Let us prove that $\tilde{\psi}$ is injective on $\mathcal{T}_{\mathcal{E}}^{e} / J_{e}$. First, pick $b \in \mathcal{T}_{\mathcal{E}}^{e}$ of the form

$$
\tilde{t}\left(\xi_{p_{1}}\right) \tilde{t}\left(\xi_{p_{2}}\right)^{*} \ldots \tilde{t}\left(\xi_{p_{2 k-1}}\right) \tilde{t}\left(\xi_{p_{2 k}}\right)^{*}
$$

with $k \in \mathbb{N}, p_{1} p_{2}^{-1} \ldots p_{2 k-1} p_{2 k}^{-1}=e$ and $\xi_{p_{i}} \in \mathcal{E}_{p_{i}}$ for all $i \in\{1,2, \ldots, 2 k\}$. Assume that $q(b) \neq 0$. This entails

$$
K_{\left\{p_{2 i}, p_{2 i+1}\right\}}=p_{2 i} P \cap p_{2 i+1} P \neq \emptyset
$$

for each $i \in\{1,2, \ldots, k-1\}$ because, otherwise, $t_{F}\left(\tilde{t}\left(\xi_{p_{2 i}}\right) * \tilde{t}\left(\xi_{p_{2 i+1}}\right)\right)$ acts trivially on $\mathcal{E}_{F}^{+}$, which would imply $q(b)=0$. A similar argument employed to

$$
F:=\left\{p_{2 k}, p_{2 k} p_{2 k-1}^{-1} p_{2 k-2}, p_{2 k} p_{2 k-1}^{-1} p_{2 k-2} p_{2 k-3}^{-1} p_{2 k-4}, \ldots, p_{2 k} p_{2 k-1}^{-1} p_{2 k-2} \cdots p_{3}^{-1} p_{2}\right\}
$$

shows that $K_{F} \neq \emptyset$. This is precisely the right ideal

$$
p_{2 k} p_{2 k-1}^{-1} p_{2 k-2} \cdots p_{3}^{-1} p_{2} P
$$

These ideals are used in 39 to study semigroup $\mathrm{C}^{*}$-algebras.

We claim that, if $r \notin \bar{K}_{F}$, then

$$
\widetilde{\psi}(b)\left(\psi_{r}\left(\mathcal{E}_{r} I_{r^{-1}(r \vee F)}\right)\right)=0 \text {. }
$$

Since $\psi$ is strongly covariant, it suffices to prove that $t_{\{e\}}(b) t_{\{e\}}^{r}\left(\mathcal{E}_{r} I_{r-1}(r \vee F)\right)$ vanishes on $\mathcal{E}_{\{e\}}^{+}$. First, notice that $t_{\{e\}}(b)=0$ on $\mathcal{E}_{s} I_{s^{-1}(s \vee g)}$ whenever $s \notin K_{F}$. Hence if $K_{\{r, F\}}=\emptyset$, it follows that $t_{\{e\}}(b) t_{\{e\}}^{r}\left(\mathcal{E}_{r}\right)=\{0\}$ because the image of $\mathcal{E}_{r}$ under $t_{\{e\}}^{r}$ sends $\mathcal{E}_{s} I_{s^{-1}(s \vee g)}$ to $\mathcal{E}_{r s} I_{s^{-1} r^{-1}(r s \vee r g)}$ 


\section{6. $\mathrm{C}^{*}$-ALGEBRAS FOR PRODUCT SYSTEMS OVER SUBSEMIGROUPS OF GROUPS}

and $t_{\{e\}}(b)$ vanishes on the latter. We are then left with the case in which $K_{\{r, F\}} \neq \emptyset$. Thus $r \notin K_{F}$ implies that $r \notin K_{\{g\}}$ for some $g \in F$. Let $\xi_{r} \in \mathcal{E}_{r} I_{r^{-1}(r \vee F)}$. Then $t_{\{e\}}(b) t_{\{e\}}^{r}\left(\xi_{r}\right)$ vanishes on the direct summand $\mathcal{E}_{s} I_{s^{-1}(s \vee g)}$ if $r s \notin K_{F}$. So assume that $r s \in K_{F}$. In particular, $r s \in K_{\{r, g\}}$. Hence $I_{r^{-1}(r \vee F)} \subseteq \operatorname{ker} \varphi_{s}$ and $t_{\{e\}}^{r}\left(\xi_{r}\right)=0$ on the direct summand $\mathcal{E}_{s} I_{s^{-1}(s \vee g)}$. This concludes the proof that $t_{\{e\}}(b) t_{\{e\}}^{r}\left(\xi_{r}\right)=0$ on $\mathcal{E}_{\{e\}}^{+}$. Therefore, $\widetilde{\psi}(b) \psi_{r}\left(\mathcal{E}_{r} I_{r^{-1}(r \vee F)}\right)=0$ as claimed.

Now let $b \in \mathcal{T}_{\mathcal{E}}^{e}$ be such that $\widetilde{\psi}(b)=0$. Given $\varepsilon>0$, we must find a finite set $F \subseteq G$ such that $\|b\|_{F}<\varepsilon$. By Lemma 4.1.6 there exists $b^{\prime}=\sum_{j=1}^{n} b_{j} \in \mathcal{T}_{\mathcal{E}}^{e}$ with $\left\|b-b^{\prime}\right\|<\frac{\varepsilon}{2}$, where each $b_{j}$ is of the form (6.2.2). For each $j \in\{1, \ldots, n\}$, let $F_{j} \subseteq G$ be the finite set associated to $b_{j}$ as in (6.2.3). Thus $\widetilde{\psi}\left(b_{j}\right)\left(\psi_{r}\left(\mathcal{E}_{r} I_{r^{-1}\left(r \vee F_{j}\right.}\right)\right)=0$ if $r \notin K_{F_{j}}$. We also set

$$
F:=\bigcup_{j=1}^{m} F_{j}
$$

and let $\xi=\bigoplus_{r \in P} \xi_{r} \in \mathcal{E}_{F}$ with $\|\xi\|_{F} \leq 1$, where $\xi_{r}=0$ except for finitely many $r$ 's. Then

$$
\left\|\sum \psi_{r}\left(\xi_{r}\right)^{*} \widetilde{\psi}\left(b^{\prime}\right)^{*} \widetilde{\psi}\left(b^{\prime}\right) \psi_{r}\left(\xi_{r}\right)\right\|=\left\|\sum \psi_{t}\left(\xi_{t}\right)^{*} \widetilde{\psi}\left(b-b^{\prime}\right)^{*} \widetilde{\psi}\left(b-b^{\prime}\right) \psi_{r}\left(\xi_{r}\right)\right\|<\frac{\varepsilon^{2}}{4} .
$$

Since $\psi_{e}$ is injective and $\widetilde{\psi}\left(b_{j}\right) \psi_{r}\left(\xi_{r}\right)=0$ if $r \notin K_{F_{j}}$, it follows that the left-hand-side above is precisely $\left\|t_{F}\left(b^{\prime}\right)(\xi)\right\|_{e}^{2}$. This implies that $\|b\|_{F}<\varepsilon$. Hence $b$ belongs to $J_{e}$ as desired.

Lemma 6.2.4. Let $G$ and $H$ be groups containing $P$ as a subsemigroup and let $\mathcal{E}$ be a product system over $P$. A representation of $\mathcal{E}$ is strongly covariant as in Definition 6.1.2 with respect to $G$ if and only if it is strongly covariant with respect to $H$. Thus different groups provide the same notion of strong covariance.

Proof. We may assume that $G=G(P)$ is the universal group of $P$. By its universal property, there is a group homomorphism $\gamma: G \rightarrow H$ extending the embedding of $P$ into $H$. Let $e_{G}$ and $e_{H}$ denote the unit elements of $G$ and $H$, respectively. Let $\mathcal{T}_{\mathcal{E}}^{e_{G}}$ be the fixed-point algebra for the generalised gauge coaction of $G$ on $\mathcal{T}_{\mathcal{E}}$. It follows from Lemma 4.1.6 that $\mathcal{T}_{\mathcal{E}}^{e_{G}}$ is a $\mathrm{C}^{*}$-subalgebra of $\mathcal{T}_{\mathcal{E}}^{e_{H}}$, where $\mathcal{T}_{\mathcal{E}}^{e_{H}}$, in turn, is the fixed-point algebra for the gauge coaction of $H$ on $\mathcal{T}_{\mathcal{E}}$. Let us prove that $J_{e_{G}} \subseteq J_{e_{H}}$, where $J_{e_{G}} \triangleleft \mathcal{T}_{\mathcal{E}}^{e_{G}}$ and $J_{e_{H}} \triangleleft \mathcal{T}_{\mathcal{E}}^{e_{H}}$ are the ideals described in the construction before Definition 6.1.2 with respect to the groups $G$ and $H$, respectively.

Indeed, it suffices to show that, given a finite set $F$ with $F \subseteq G$, one has

$$
I_{p^{-1}(p \vee F)} \supseteq I_{\gamma(p)^{-1}(\gamma(p) \vee \gamma(F))},
$$

where $\gamma(F)$ is the range of $F$ under $\gamma$. To do so, let $g \in F$. If either $K_{\{p, g\}}=\emptyset$ or $p \in K_{\{p, g\}}$, then $I_{p^{-1}(p \vee g)}=A \supseteq I_{\gamma(p)^{-1}(\gamma(p) \vee \gamma(g))}$.

Suppose that $K_{\{p, g\}} \neq \emptyset$ and $p \notin K_{\{p, g\}}$. Given $r \in K_{\{p, g\}}, \gamma(r) \in K_{\{\gamma(p), \gamma(g)\}}$ so that $\operatorname{ker} \varphi_{p^{-1} r} \supseteq I_{\gamma(p)^{-1}(\gamma(p) \vee \gamma(g))}$ because $\gamma$ is a group homomorphism. So we conclude that $I_{p^{-1}(p \vee F)} \supseteq$ $I_{\gamma(p)^{-1}(\gamma(p) \vee \gamma(F))}$ and hence $J_{e_{G}} \subseteq J_{e_{H}}$ as asserted.

Thus we obtain a ${ }^{*}$-homomorphism $\phi: \mathcal{T}_{\mathcal{E}} / J_{\infty}^{G} \rightarrow \mathcal{T}_{\mathcal{E}} / J_{\infty}^{H}$. Combining Proposition 6.1.5 with Lemma 6.2.1. we deduce that $\phi$ is injective on $\mathcal{T}_{\mathcal{E}}^{e_{G}} / J_{e_{G}}$. To see that $\phi$ is an isomorphism, we will show that $J_{e_{H}} \subseteq J_{\infty}^{G}$.

First, let $b \in \mathcal{T}_{\mathcal{E}}^{e_{H}}$ be of the form 6.2.2), that is,

$$
b=\tilde{t}\left(\xi_{p_{1}}\right) \tilde{t}\left(\xi_{p_{2}}\right)^{*} \ldots \tilde{t}\left(\xi_{p_{2 k-1}}\right) \tilde{t}\left(\xi_{p_{2 k}}\right)^{*},
$$

with $k \in \mathbb{N}, \gamma\left(p_{1}\right) \gamma\left(p_{2}\right)^{-1} \ldots \gamma\left(p_{2 k-1}\right) \gamma\left(p_{2 k}\right)^{-1}=e_{H}$ and $\xi_{p_{i}} \in \mathcal{E}_{p_{i}}$ for all $i \in\{1,2, \ldots, 2 k\}$. We claim that $p_{1} p_{2}^{-1} \ldots p_{2 k-1} p_{2 k}^{-1} \neq e_{G}$ in $G$ entails $b \in J_{e_{H}} \cap J_{\infty}^{G}$. To see this, we will prove that $K_{F}=K_{\gamma(F)}=\emptyset$, where

$$
F:=\left\{p_{2 k}, p_{2 k} p_{2 k-1}^{-1} p_{2 k-2}, \ldots, p_{2 k} p_{2 k-1}^{-1} p_{2 k-2} \cdots p_{3}^{-1} p_{2}\right\}
$$

is the finite subset of $G$ associated to $b$.

Let $r \in K_{\gamma(F)}$. Then there is a unique $s_{1} \in P$ with $r=p_{2 k} s_{1}$. Here we have omitted $\gamma$ because it is injective on $P$. Now $r$ also lies in $\gamma\left(p_{2 k} p_{2 k-1}^{-1} p_{2 k-2}\right) P$. So there is a unique $s_{2} \in P$ so that $r=\gamma\left(p_{2 k} p_{2 k-1}^{-1} p_{2 k-2}\right) s_{2}$. This implies that $\gamma\left(p_{2 k-1}^{-1} p_{2 k-2}\right) s_{2}=s_{1}$. This is so if and only $p_{2 k-2} s_{2}=$ 
$p_{2 k-1} s_{1}$. Hence $r=p_{2 k} p_{2 k-1}^{-1} p_{2 k-2} s_{2}$ in $G$ as well. Repeating this procedure, we deduce that $r \in K_{F}$. Thus $K_{\gamma(F)}=K_{F}$, since the inclusion $K_{F} \subseteq K_{\gamma(F)}$ is clear.

It remains to show that $K_{F}=K_{\gamma(F)}=\emptyset$. Let us argue by contradiction and suppose that $K_{F}$ is a non-empty subset of $G$. Hence one can find $r, s \in P$ with

$$
p_{2 k} p_{2 k-1}^{-1} p_{2 k-2} \cdots p_{3}^{-1} p_{2} p_{1}^{-1}=r\left(p_{1} s\right)^{-1} .
$$

Since $\gamma$ is injective on $P$ and $\gamma\left(p_{2 k} p_{2 k-1}^{-1} p_{2 k-2} \cdots p_{3}^{-1} p_{2} p_{1}^{-1}\right)=e_{H}$, it follows that $p_{1} s=r$. This gives $g r=r$ and thus $g=e_{G}$, contradicting our assumption that $p_{1} p_{2}^{-1} \ldots p_{2 k-1} p_{2 k}^{-1} \neq e_{G}$ in $G$. Therefore, $K_{F}=K_{\gamma(F)}=\emptyset$ and hence $b \in J_{e_{H}} \cap J_{\infty}^{G}$.

As a consequence, the image of $\mathcal{T}_{\mathcal{E}}^{e_{H}}$ under the quotient map $q: \mathcal{T}_{\mathcal{E}} \rightarrow \mathcal{T}_{\mathcal{E}} / J_{\infty}^{G}$ lies in the fixed-point algebra $\mathcal{T}_{\mathcal{E}}^{e_{G}} / J_{e_{G}}$. Since $\phi$ is faithful on this latter $\mathrm{C}^{*}$-algebra and the quotient map $\mathcal{T}_{\mathcal{E}} \rightarrow \mathcal{T}_{\mathcal{E}} / J_{\infty}^{H}$ is precisely the composite $\phi \circ q$, we conclude that $J_{e_{H}} \subseteq J_{\infty}^{G}$. Therefore $J_{\infty}^{G}=J_{\infty}^{H}$. This shows that the notion of covariance described in Definition 6.1.2 is independent of the choice of the group containing $P$ as a subsemigroup.

The following is the main result of this chapter:

Theorem 6.2.5. Let $P$ be a unital semigroup and let $\mathcal{E}=\left(\mathcal{E}_{p}\right)_{p \in P}$ be a product system over $P$ of $A$-correspondences. Suppose that $P$ is embeddable into a group. There is a $\mathrm{C}^{*}$-algebra $A \times{ }_{\mathcal{E}} P$ associated to $\mathcal{E}$ with a representation $j_{\mathcal{E}}: \mathcal{E} \rightarrow A \times_{\mathcal{E}} P$ such that the pair $\left(A \times_{\mathcal{E}} P, j_{\mathcal{E}}\right)$ has the following properties:

(C1) $A \times{ }_{\mathcal{E}} P$ is generated by $j_{\mathcal{E}}(\mathcal{E})$ as a $\mathrm{C}^{*}$-algebra and $j_{\mathcal{E}}$ is strongly covariant in the sense of Definition 6.1.2, where the group $G$ in question may be taken to be any group containing $P$ as a subsemigroup.

$(\mathrm{C} 2)$ if $\psi=\left\{\psi_{p}\right\}_{p \in P}$ is a strongly covariant representation of $\mathcal{E}$ in a $\mathrm{C}^{*}$-algebra $B$ with respect to a group containing $P$, then there is a unique ${ }^{*}$-homomorphism $\widehat{\psi}: A \times{ }_{\mathcal{E}} P \rightarrow B$ such that $\widehat{\psi} \circ j_{p}=\psi_{p}$ for all $p \in P$;

(C3) $j_{e}$ is faithful and if $G$ is a group with $P \subseteq G$ as a semigroup, there is a canonical full coaction of $G$ on $A \times{ }_{\mathcal{E}} P$ so that $a^{*}$-homomorphism $A \times{ }_{\mathcal{E}} P \rightarrow B$ is faithful on the fixed-point algebra $\left(A \times{ }_{\mathcal{E}} P\right)^{\delta}$ if and only if it is faithful on $j_{e}(A)$. (C3).

Moreover, up to canonical isomorphism, $\left(A \times_{\mathcal{E}} P, j_{\mathcal{E}}\right)$ is the unique pair with the properties $(\mathrm{C} 1)-$

Proof. Let $G$ be a group containing $P$ as a subsemigroup. Let $J_{\infty}$ be the ideal in $\mathcal{T}_{\mathcal{E}}$ as in Lemma 6.1 .3 That is, $J_{\infty}$ is the ideal generated by $J_{e}$, which in turn is the ideal in $\mathcal{T}_{\mathcal{E}}^{e}$ constructed before Definition 6.1.2. Set $A \times_{\mathcal{E}} P:=\mathcal{T}_{\mathcal{E}} / J_{\infty}$ and let $j_{\mathcal{E}}$ be the representation of $\mathcal{E}$ in $A \times{ }_{\mathcal{E}} P$ given by the composition of $\tilde{t}: \mathcal{E} \rightarrow \mathcal{T}_{\mathcal{E}}$ with the quotient map $q: \mathcal{T}_{\mathcal{E}} \rightarrow \mathcal{T}_{\mathcal{E}} / J_{\infty}$. By Lemma 6.2.4 this does not depend on the chosen group and hence it satisfies $(\mathrm{C} 1)$. By the universal property of $\mathcal{T}_{\mathcal{E}}$ and again by Lemma 6.2.4 $A \times_{\mathcal{E}} P$ also fulfils (C2). Now (C3) follows from Lemma 6.2.1 Uniqueness of $\left(A \times{ }_{\mathcal{E}} P, j_{\mathcal{E}}\right)$ is then clear.

We call $A \times{ }_{\mathcal{E}} P$ the covariance algebra of $\mathcal{E}$, following the terminology of [32] for $\mathrm{C}^{*}$-algebras associated to partial dynamical systems.

Remark 6.2.6. The proof of Lemma 6.2.4 also tells us that the fixed-point algebras of the canonical coactions on $A \times{ }_{\mathcal{E}} P$ of all groups containing $P$ coincide.

Example 6.2.7. Let $G$ be a group and $\left(B_{g}\right)_{g \in G}$ a saturated Fell bundle over $G$. View $\left(B_{g}\right)_{g \in G}$ as a product system over $G$. For each $g \in G$ and $F \subseteq G$ finite, we have that $I_{g^{-1}(g \vee F)}=B_{e}$ since $g \in K_{\{g, h\}}$ for all $h \in F$. Hence $J_{e}=\{0\}$ and the associated covariance algebra is isomorphic to the cross sectional $\mathrm{C}^{*}$-algebra of $\left(B_{g}\right)_{g \in G}$.

\subsection{Relationship to other constructions}

In this section, we relate the covariance algebras of product systems defined here to other constructions in the setting of irreversible dynamical systems. We also give an equivalent notion of strongly covariant representations for compactly aligned product systems over quasi-lattice ordered groups. 


\subsubsection{Relationship to a construction by Sims and Yeend}

Let us restrict our attention to compactly aligned product systems over positive cones of quasi-lattice orders. In [55], Sims and Yeend constructed a $\mathrm{C}^{*}$-algebra $\mathcal{N} \mathcal{O}_{\mathcal{E}}$ from a compactly aligned product system $\mathcal{E}=\left(\mathcal{E}_{p}\right)_{p \in P}$ so that it generalises constructions such as $\mathrm{C}^{*}$-algebras associated to finitely aligned higher rank graphs and Katsura's Cuntz-Pimsner algebra of a single correspondence. The universal representation of $\mathcal{E}$ in $\mathcal{N} \mathcal{O}_{\mathcal{E}}$ is quite often faithful, but Example 3.16 of [55] shows that it may fail to be injective even if $(G, P)$ is totally ordered and $A$ acts by compact operators on $\mathcal{E}_{p}$ for all $p$ in $P$. In this subsection, we will see that $\mathcal{N} \mathcal{O}_{\mathcal{E}}$ coincides with $A \times_{\mathcal{E}} P$ when either the universal representation of $\mathcal{E}$ in $\mathcal{N} \mathcal{O}_{\mathcal{E}}$ is faithful and $P$ is directed or $\mathcal{E}$ is a faithful product system. In both cases $\mathcal{N} \mathcal{O}_{\mathcal{E}}$ satisfies an analogue of (C3) [15. Proposition 3.7]. This subsection is based on [15] and [55].

We first recall the definitions from [55] of Cuntz-Nica-Pimsner covariance and Cuntz-Nica-Pimsner algebra. Fix a quasi-lattice ordered group $(G, P)$ and let $\mathcal{E}=\left(\mathcal{E}_{p}\right)_{p \in P}$ be a compactly aligned product system over $P$. Let $\bar{I}_{e}:=A$ and, for each $p \in P \backslash\{e\}$, set

$$
\bar{I}_{p}=\bigcap_{e<s \leq p} \operatorname{ker} \varphi_{s} \triangleleft A .
$$

Given $p \in P$, we define a correspondence $\widetilde{\mathcal{E}}_{p}: A \leadsto A$ by

$$
\widetilde{\mathcal{E}}_{p}:=\bigoplus_{r \leq p} \mathcal{E}_{r} \bar{I}_{r^{-1} p}
$$

For all $s \in P$, there is a ${ }^{*}$-homomorphism $\widetilde{\iota}_{s}^{p}: \mathbb{B}\left(\mathcal{E}_{s}\right) \rightarrow \mathbb{B}\left(\widetilde{\mathcal{E}}_{p}\right)$ defined by

$$
\tilde{\iota}_{s}^{p}(T)=\left(\left.\bigoplus_{s \leq r \leq p} \iota_{s}^{r}(T)\right|_{\mathcal{E}_{r} \bar{I}_{r-1} p}\right) \oplus\left(\bigoplus_{s \leq r \leq p} 0_{\mathcal{E}_{r} \bar{I}_{r-1}}\right) \quad \text { for all } T \in \mathbb{B}\left(\mathcal{E}_{s}\right) .
$$

Let $F \subseteq P$ be a finite set and let $T_{s} \in \mathbb{K}\left(\mathcal{E}_{s}\right)$ for each $s \in F$. We say that $\sum_{s \in F} \widetilde{\iota}_{s}^{p}\left(T_{s}\right)=0$ for large $p$ if given an arbitrary element $r$ in $P$, there exists $r^{\prime} \geq r$ such that $\sum_{s \in F} \widetilde{\iota}_{s}^{p}\left(T_{s}\right)=0$ for all $p \geq r^{\prime}$. A representation $\psi$ of $\mathcal{E}$ in a $\mathrm{C}^{*}$-algebra $B$ is Cuntz-Pimsner covariant according to 55 , Definition $3.9]$ if

$$
\sum_{s \in F} \psi^{(s)}\left(T_{s}\right)=0
$$

whenever $\sum_{s \in F} \widetilde{\iota}_{s}^{p}\left(T_{s}\right)=0$ for large $p$. It is called Cuntz-Nica-Pimsner covariant if it is both Nica covariant and Cuntz-Pimsner covariant.

Suppose that $\mathcal{E}$ is a product system with the extra property that $\widetilde{\iota}_{e}^{p}$ is injective on $A$ for all $p \in P$. The Cuntz-Nica-Pimsner algebra associated to $\mathcal{E}$, denoted by $\mathcal{N} \mathcal{O}_{\mathcal{E}}$, is then the universal $\mathrm{C}^{*}$-algebra for Cuntz-Nica-Pimsner covariant representations (see [55, Proposition 3.2] for further details). The requirement that $\widetilde{\iota}_{e}^{p}$ be faithful for all $p \in P$ implies that the representation of $\mathcal{E}$ in $\mathcal{N} \mathcal{O}_{\mathcal{E}}$ is faithful. Sims and Yeend proved in [55. Lemma 3.15] that this is satisfied whenever $P$ has the following property: given a non-empty set $F \subseteq P$ that is bounded above, in the sense that there is $p \in P$ with $s \leq p$ for all $s \in F$, then $F$ has a maximal element $r$. That is, $r \not \leq s$ for all $s \in F \backslash\{r\}$.

The next example of a product system is given by Sims and Yeend in [55, Example 3.16]. It consists of a compactly aligned product system for which not all $\widetilde{\iota}_{e}^{p}$ 's are injective. We recall their example here and describe its associated covariance algebra.

Example 6.3.1. Let $\mathbb{Z} \times \mathbb{Z}$ be equipped with the lexicographic order and let $P$ be its positive cone. So $P=((\mathbb{N} \backslash\{0\}) \times \mathbb{Z}) \cup(\{0\} \times \mathbb{N})$ and $e=\{0\} \times\{0\}$. Define a product system over $P$ as follows: let $A:=\mathbb{C}^{2}$ and, for each $p \in P$, let $\mathcal{E}_{p}:=\mathbb{C}^{2}$ be regarded as a Hilbert $A$-module with right action given by coordinatewise multiplication and usual $\mathbb{C}^{2}$-valued inner-product. Following the notation of $\left[55\right.$, we set $S:=\{0\} \times \mathbb{N}$ and for all $p \in S$, we let $\mathbb{C}^{2}$ act on $\mathcal{E}_{p}$ on the left by coordinatewise multiplication, so that $\varphi_{p}\left(\left(\lambda_{1}, \lambda_{2}\right)\right):=\left(\lambda_{1}, \lambda_{2}\right) \in \mathbb{B}\left(\mathcal{E}_{p}\right)$ for all $p \in P$ and $\left(\lambda_{1}, \lambda_{2}\right) \in A$. For $p \in P \backslash S$, put $\varphi_{p}\left(\left(\lambda_{1}, \lambda_{2}\right)\right):=\left(\lambda_{1}, \lambda_{1}\right)$. Thus ker $\varphi_{p}=\{0\} \times \mathbb{C}$ for all $p \in P \backslash S$. If $q \in S$, define a correspondence isomorphism $\mu_{p, q}: \mathcal{E}_{p} \otimes_{\mathbb{C}^{2}} \mathcal{E}_{q} \cong \mathcal{E}_{p q}$ by

$$
\left(z_{1}, z_{2}\right) \otimes\left(w_{1}, w_{2}\right) \mapsto\left(z_{1} w_{1}, z_{2} w_{2}\right) .
$$


For all $q \in P \backslash S$, define $\mu_{p, q}: \mathcal{E}_{p} \otimes_{\mathbb{C}^{2}} \mathcal{E}_{q} \cong \mathcal{E}_{p q}$ by

$$
\left(z_{1}, z_{2}\right) \otimes\left(w_{1}, w_{2}\right) \mapsto\left(z_{1} w_{1}, z_{1} w_{2}\right) .
$$

This is a proper product system $\mathcal{E}=\left(\mathcal{E}_{p}\right)_{p \in P}$ over $\mathbb{C}^{2}$. Thus it is also compactly aligned. Since $P$ is totally ordered, all representations of $\mathcal{E}$ are Nica covariant. Sims and Yeend proved that such a product system has no injective Cuntz-Nica-Pimsner covariant representation. Their argument is the following: for all $p \neq e, \bar{I}_{p}=\operatorname{ker} \varphi_{(0,1)}=\{0\}$. Hence, if $q \in P \backslash S, \widetilde{\iota}_{e}^{q}=\varphi_{q}$ is not injective and any Cuntz-Nica-Pimsner covariant representation of $\mathcal{E}$ vanishes on $\operatorname{ker} \varphi_{q}=\{0\} \times \mathbb{C}$.

Let us now describe the associated covariance algebra $A \times_{\mathcal{E}} P$. We will show that $\left(A \times_{\mathcal{E}} P\right)^{\delta}$ is isomorphic to the $\mathrm{C}^{*}$-algebra of all convergent sequences. To do so, given $p \in P$, write $p=\left(p_{1}, p_{2}\right)$. We define an isometry $v_{p} \in \mathbb{B}\left(\ell^{2}(\mathbb{N} \times \mathbb{Z})\right)$ by

$$
v_{p}(f)(q)= \begin{cases}f(q-p) & \text { if } q_{1} \geq p_{1} \\ 0 & \text { otherwise }\end{cases}
$$

where $f \in \ell^{2}(\mathbb{N} \times \mathbb{Z})$ and $q=\left(q_{1}, q_{2}\right) \in \mathbb{N} \times \mathbb{Z}$. Thus $v_{p}^{*}(f)(q)=f(q+p)$ and $v_{p} v_{p}^{*}$ is the projection of $\ell^{2}(\mathbb{N} \times \mathbb{Z})$ onto the subspace $\ell^{2}\left(\mathbb{N}_{\geq p_{1}} \times \mathbb{Z}\right)$. In particular, $v_{p}$ is unitary for all $p \in S$.

Let $\phi_{e}: \mathbb{C}^{2} \rightarrow \mathbb{B}\left(\ell^{2}(\mathbb{N} \times \mathbb{Z})\right)$ be the ${ }^{*}$-homomorphism given by

$$
\phi_{e}\left(\left(\lambda_{1}, \lambda_{2}\right)\right)(f)(q)= \begin{cases}\lambda_{2} f(q) & \text { if } q_{1}=0 \\ \lambda_{1} f(q) & \text { otherwise }\end{cases}
$$

For all $\left(z_{1}, z_{2}\right)_{p} \in \mathcal{E}_{p}$, put $\phi_{p}\left(\left(z_{1}, z_{2}\right)_{p}\right):=v_{p} \phi_{e}\left(\left(z_{1}, z_{2}\right)\right)$. This yields a representation $\phi=\left\{\phi_{p}\right\}_{p \in P}$ of $\mathcal{E}$ in $\mathbb{B}\left(\ell^{2}(\mathbb{N} \times \mathbb{Z})\right)$. We claim that $\phi$ is strongly covariant and preserves the topological $\mathbb{Z} \times \mathbb{Z}$-grading of $A \times_{\mathcal{E}} P$. First, for each finite set $F \subseteq P$,

$$
B_{F}:=\operatorname{span}\left\{T_{p} \mid p \in F, T_{p} \in \mathbb{K}\left(\mathcal{E}_{p}\right)\right\}
$$

is a $\mathrm{C}^{*}$-subalgebra of $\mathcal{T}_{\mathcal{E}}^{e}$ since $P$ is totally ordered (see also [15, Lemma 3.6]). In addition, $\mathcal{T}_{\mathcal{E}}^{e}=$ $\overline{\bigcup_{F \subseteq P} B_{F}}$. By 3 , Lemma 1.3],

$$
J_{e}=\overline{\bigcup_{F \subseteq P} J_{e} \cap B_{F}} .
$$

So in order to prove that $\phi$ is strongly covariant, it suffices to verify that, given a finite set $F \subseteq P$, one has

$$
\sum_{p \in F} \phi^{(p)}\left(T_{p}\right)=0
$$

whenever $\sum_{p \in F} j_{\mathcal{E}}^{(p)}\left(T_{p}\right)=0$ in $A \times_{\mathcal{E}} P$. Here $T_{p} \in \mathbb{K}\left(\mathcal{E}_{p}\right)$ for each $p \in F$. So suppose that $F$ is a finite subset of $P$ and $\sum_{p \in F} j_{\mathcal{E}}^{(p)}\left(T_{p}\right)=0$ in $A \times_{\mathcal{E}} P$, with $T_{p} \in \mathbb{K}\left(\mathcal{E}_{p}\right)$. Let $\left(\lambda_{1, p}, \lambda_{2, p}\right)$ be such that $T_{p}=\left(\lambda_{1, p}, \lambda_{2, p}\right)$. Write

$$
F=\bigcup_{i=1}^{n} F_{p_{i}}
$$

with $p_{i}<p_{i+1}$ for all $i \in\{1, \ldots, n\}$, where $F_{p_{i}}$ is given by all of the elements in $F$ having first component $p_{i}$. Given a finite set $F^{\prime} \subseteq P$ with $F^{\prime} \supseteq F$, there is $r_{1}=\left(p_{1}, q_{1}\right) \in P$ such that $p^{\prime}<r_{1}$ for all $p^{\prime} \in F_{p_{1}}^{\prime}$. Then

$$
\mathcal{E}_{r_{1}} I_{r_{1}^{-1}\left(r_{1} \vee F^{\prime}\right)}=\{0\} \times \mathbb{C} .
$$

So by taking finite sets $F^{\prime} \subseteq P$ with $F^{\prime} \supseteq F$, we conclude from the definition of strong covariance that

$$
\sum_{p \in F_{p_{1}}} \lambda_{2, p}=0
$$

If $p_{2}>p_{1}+1$, we deduce by a similar argument that the sum $\sum_{p \in F_{p_{1}}} \lambda_{1, p}$ must be zero as well because

$$
\iota_{p}^{r}\left(T_{p}\right)\left(\lambda_{1}, \lambda_{2}\right)=\iota_{p}^{r}\left(\left(\lambda_{1, p}, \lambda_{2, p}\right)\right)\left(\lambda_{1}, \lambda_{2}\right)=\left(\lambda_{1, p} \lambda_{1}, \lambda_{1, p} \lambda_{2}\right)
$$


for all $r=\left(r_{1}, r_{2}\right)>p$ with $r_{1}>p_{1}$. In case $p_{2}=p_{1}+1$, then

$$
\sum_{p \in F_{p_{1}}} \lambda_{1, p}+\sum_{p \in F_{p_{2}}} \lambda_{2, p}=0
$$

Repeating this argument for all of the $p_{i}$ 's and observing that

$$
\phi^{(p)}\left(T_{p}\right)(f)(q)= \begin{cases}\lambda_{2, p} f(q) & \text { if } q_{1}=p_{1} \\ \lambda_{1, p} f(q) & \text { if } q_{1}>p_{1} \\ 0 & \text { otherwise }\end{cases}
$$

we conclude that $\phi$ is indeed strongly covariant. The associated representation of $A \times \mathcal{E} P$ on $\ell^{2}(\mathbb{N} \times \mathbb{Z})$ is faithful on $\left(A \times{ }_{\mathcal{E}} P\right)^{\delta}$ because it is injective on $\mathbb{C}^{2}$. Its image in $\mathbb{B}\left(\ell^{2}(\mathbb{N} \times \mathbb{Z})\right)$ is the $\mathrm{C}^{*}$-algebra generated by $\phi_{e}\left(\mathbb{C}^{2}\right)$ and the family of isometries $\left\{v_{p} \mid p \in P\right\}$.

To see that $\widehat{\phi}$ is faithful on $A \times{ }_{\mathcal{E}} P$, consider the canonical unitary representation of the torus $\mathbb{T}^{2}$ on $\ell^{2}(\mathbb{N} \times \mathbb{Z})$. Explicitly, the unitary $U_{z}$ is given by

$$
U_{z}(f)(q)=z_{1}^{q_{1}} z_{2}^{q_{2}} f(q), \quad q=\left(q_{1}, q_{2}\right) \in \mathbb{N} \times \mathbb{Z},
$$

where $z=\left(z_{1}, z_{2}\right) \in \mathbb{T}^{2}$. This produces a continuous action of $\mathbb{T}^{2}$ on $\widehat{\phi}\left(A \times{ }_{\mathcal{E}} P\right)$ by $T \mapsto U T U^{*}$. Hence it carries a topological $\mathbb{Z} \times \mathbb{Z}$-grading (see Section 2.2 ). The corresponding spectral subspace at $(m, n)$ is determined by

$$
\left\{T \in \widehat{\phi}\left(A \times{ }_{\mathcal{E}} P\right) \mid U_{z} T U_{z}^{*}=z_{1}^{m} z_{2}^{n} T \text { for all } z=\left(z_{1}, z_{2}\right) \in \mathbb{T}^{2}\right\} .
$$

Since $\mathbb{Z} \times \mathbb{Z}=P \cup P^{-1}$, it is easy to verify that $\widehat{\phi}$ preserves the grading of $A \times{ }_{\mathcal{E}} P$. Because $\mathbb{Z} \times \mathbb{Z}$ is amenable, $\widehat{\phi}$ is then an isomorphism onto its image. Its restriction to $\left(A \times_{\mathcal{E}} P\right)^{\delta}$ yields an isomorphism onto the $\mathrm{C}^{*}$-algebra of all convergent sequences

$$
\left\{\left(\zeta_{n}\right)_{n \in \mathbb{N}} \in \ell^{\infty}(\mathbb{N}) \mid \exists \lim _{n \rightarrow \infty} \zeta_{n}\right\} .
$$

This isomorphism sends $\left(\lambda_{1}, \lambda_{2}\right)_{p} \in \mathbb{K}\left(\mathcal{E}_{p}\right)$ to the sequence $\left(\zeta_{n}\right)_{n \in \mathbb{N}}$ with

$$
\zeta_{n}= \begin{cases}\lambda_{2} & \text { if } n=p_{1} \\ \lambda_{1} & \text { if } n>p_{1} \\ 0 & \text { otherwise }\end{cases}
$$

The task of verifying whether a given representation is strongly covariant or not is considerably simplified when $\mathcal{E}$ is compactly aligned. The proof of the next proposition is taken from 15. Proposition $3.7]$ and adapted to our context.

Proposition 6.3.2. Let $\mathcal{E}=\left(\mathcal{E}_{p}\right)_{p \in P}$ be a compactly aligned product system. A representation $\psi$ of $\mathcal{E}$ in a $\mathrm{C}^{*}$-algebra $B$ is strongly covariant if and only if it is Nica covariant and satisfies

(C)' $\sum_{p \in F} \psi^{(p)}\left(T_{p}\right)=0$ whenever $\sum_{p \in F} t_{F}^{(p)}\left(T_{p}\right)=0$ on $\mathcal{E}_{F}$, where $F \subseteq P$ is finite and $T_{p} \in \mathbb{K}\left(\mathcal{E}_{p}\right)$ for all $p \in F$.

Proof. Suppose that $\psi$ is strongly covariant. Let $p, q \in P, T \in \mathbb{K}\left(\mathcal{E}_{p}\right)$ and $S \in \mathbb{K}\left(\mathcal{E}_{q}\right)$. If $p \vee q=\infty$, then $K_{\{p, q\}}=\emptyset$ so that $t_{F}^{(p)}(T) t_{F}^{(q)}(S)=0$ on the direct summand $\mathcal{E}_{F}$ for all finite subsets $F$ of $G$. Hence strong covariance implies $j^{(p)}(T) j^{(q)}(S)=0$ in $A \times_{\mathcal{E}} P$. Assume that $p \vee q<\infty$. Let $T^{\prime} \in \mathbb{K}\left(\mathcal{E}_{p \vee q}\right)$ be such that $\iota_{p}^{p \vee q}(T) \iota_{q}^{p \vee q}(S)=T^{\prime}$. Because $t_{F}^{(p)}(T) t_{F}^{(q)}(S)=0$ on $\mathcal{E}_{r} I_{r^{-1}(r \vee F)}$ whenever $r \notin(p \vee q) P$, it follows that $t_{F}^{(p)}(T) t^{(q)}(S)-t_{F}^{(p \vee q)}\left(T^{\prime}\right)=0$ for each finite subset $F$ of $G$. Hence $j^{(p)}(T) j^{(q)}(S)=j^{(p \vee q)}\left(T^{\prime}\right)$ and $j_{\mathcal{E}}$ is Nica covariant.

Now if $\sum_{p \in F} t_{F}^{(p)}\left(T_{p}\right)=0$ on $\mathcal{E}_{F}$ and $F^{\prime} \supseteq F$, then $\sum_{p \in F^{\prime}} t_{F^{\prime}}^{(p)}\left(T_{p}\right)=0$ on $\mathcal{E}_{F^{\prime}}$ since $\mathcal{E}_{F^{\prime}}^{+}$may be viewed as a closed submodule of $\mathcal{E}_{F}^{+}$(see Section 6.1 for further details). So $\sum_{p \in F} j^{(p)}\left(T_{p}\right)=0$ in $A \times_{\mathcal{E}} P$ and, in particular, $\sum_{p \in F} \psi^{(p)}\left(T_{p}\right)=0$. 
Conversely, assume that $\psi$ is Nica covariant and satisfies $(\mathrm{C})^{\prime}$. In order to prove that $\psi$ is strongly covariant, we use the ideas employed in $\left[15\right.$. Let $\mathcal{P}_{\text {fin }}^{\vee}$ denote the set of all finite subsets of $P$ that are $\vee$-closed. Precisely, $F \in \mathcal{P}_{\text {fin }}^{\vee}$ if it is finite and for all $p, q \in F$ with $p \vee q<\infty$, one has $p \vee q \in F$. For each $F$ in $\mathcal{P}_{\text {fin }}^{\vee}$, let $B_{F}$ denote the subspace of $\mathcal{N} \mathcal{T}_{\mathcal{E}}^{e}$ given by

$$
\left\{\sum_{p \in F} T_{p} \mid T_{p} \in \mathbb{K}\left(\mathcal{E}_{p}\right)\right\} .
$$

Here we introduce no special notation to identify an element of $\mathbb{K}\left(\mathcal{E}_{p}\right)$ with its image in $\mathcal{N} \mathcal{T}_{\mathcal{E}}$. We observe that $B_{F}$ is a $\mathrm{C}^{*}$-subalgebra of $\mathcal{N} \mathcal{T}_{\mathcal{E}}^{e}$ and, in addition,

$$
\mathcal{N} \mathcal{T}_{\mathcal{E}}^{e}=\overline{\bigcup_{F \in \mathcal{P}_{\text {fin }}^{\vee}} B_{F}} .
$$

Hence, since $A \times_{\mathcal{E}} P$ is a quotient of $\mathcal{N} \mathcal{T}_{\mathcal{E}}$, Lemma 1.3 of 3 says that all we must do is prove that $\sum_{p \in F} t_{F}^{(p)}\left(T_{p}\right)=0$ on $\mathcal{E}_{F}$ if $\sum_{p \in F} j^{(p)}\left(T_{p}\right)=0$ in $A \times_{\mathcal{E}} P$.

Given $r \in P$, it follows from Nica covariance that $j^{(p)}\left(T_{p}\right) j_{r}\left(\mathcal{E}_{r}\right)=0$ when $p \vee r=\infty$ and

$$
j^{(p)}\left(T_{p}\right) j_{r}\left(\mathcal{E}_{r}\right) \subseteq j_{r}\left(\mathcal{E}_{r}\right) j^{\left(r^{-1}(r \vee p)\right)}\left(\mathbb{K}\left(\mathcal{E}_{r^{-1}(r \vee p)}\right)\right)
$$

otherwise. So $j^{(p)}\left(T_{p}\right) j_{r}\left(\mathcal{E}_{r} \operatorname{ker} \varphi_{r^{-1}(r \vee p)}\right)=0$ if $p \not \leq r$. Therefore,

$$
\begin{aligned}
\sum_{p \in F} j^{(p)}\left(T_{p}\right) j_{r}\left(\mathcal{E}_{r} I_{r^{-1}(r \vee F)}\right) & =\sum_{\substack{p \in F \\
p \leq r}} j^{(p)}\left(T_{p}\right) j_{r}\left(\mathcal{E}_{r} I_{r-1}(r \vee F)\right) \\
& =j_{r}\left(\sum_{\substack{p \in F \\
p \leq r}} t_{F}^{(p)}\left(T_{p}\right) \mathcal{E}_{r} I_{r^{-1}(r \vee F)}\right) .
\end{aligned}
$$

Since $j_{\mathcal{E}}$ is injective, $\sum_{p \in F} j^{(p)}\left(T_{p}\right)=0$ yields $\sum_{\substack{p \in F \\ p \leq r}} t_{F}^{(p)}\left(T_{p}\right) \mathcal{E}_{r} I_{r^{-1}(r \vee F)}=0$ for all $r \in P$, and we deduce that $\sum_{p \in F} t_{F}^{(p)}\left(T_{p}\right)=0$ on $\mathcal{E}_{F}$ as desired.

Recall that a $\mathrm{C}^{*}$-algebra $A$ is nuclear if, for every $\mathrm{C}^{*}$-algebra $B$, there is a unique $\mathrm{C}^{*}$-norm on $A \odot B$ (see, for example, [10]). The previous proposition combined with [53, Theorem 6.3] gives us the following:

Corollary 6.3.3. Let $(G, P)$ be a quasi-lattice orderd group and let $\mathcal{E}=\left(\mathcal{E}_{p}\right)_{p \in P}$ be a compactly aligned product system. Suppose that $G$ is amenable. If $A$ is nuclear, then $A \times{ }_{\mathcal{E}} P$ is nuclear.

We denote by $q_{\mathcal{N}}$ the ${ }^{*}$-homomorphism from $\mathcal{N} \mathcal{T}_{\mathcal{E}}$ to $A \times_{\mathcal{E}} P$ induced by $j_{\mathcal{E}}=\left\{j_{p}\right\}_{p \in P}$. The proof of the next result is essentially identical to that of Proposition 6.3.2 This is inspired by [15, Proposition 3.7].

Proposition 6.3.4. Let $\psi$ be an injective Nica covariant representation of $\mathcal{E}$ in a $\mathrm{C}^{*}$-algebra $B$ and let $\psi_{\mathcal{N}}$ denote the induced ${ }^{*}$-homomorphism. Then $\left(\operatorname{ker} \psi_{\mathcal{N}}\right) \cap \mathcal{N} \mathcal{T}_{\mathcal{E}}^{e} \subseteq \operatorname{ker} q_{\mathcal{N}}$.

The following is [15, Example 3.9].

Example 6.3.5. Let $\mathbb{F}_{2}$ denote the free group on two generators $a$ and $b$. Then $\mathbb{F}_{2}$ is quasi-lattice ordered and its positive cone $\mathbb{F}_{2}^{+}$is the unital semigroup generated by $a$ and $b$. Define a product system over $\mathbb{F}_{2}^{+}$by setting $A:=\mathbb{C}, \mathcal{E}_{a}:=\mathbb{C}$ and $\mathcal{E}_{b}:=\{0\}$, where $\mathbb{C}$ is regarded as a Hilbert bimodule over $\mathbb{C}$ in the usual way. So $\mathcal{E}_{a^{n}}=\mathbb{C}$ for all $n \in \mathbb{N}$. A subset of $\mathbb{F}_{2}^{+}$that is bounded above has a maximal element, so that the representation of $\mathcal{E}$ in $\mathcal{N} \mathcal{O}_{\mathcal{E}}$ is injective. However, in 15 this example illustrates the fact that the conclusion of Proposition 6.3.4 may fail for $\mathcal{N} \mathcal{O}_{\mathcal{E}}$ if $P$ is not directed and $\mathcal{E}$ is non-faithful.

Define a representation of $\mathcal{E}$ in $\mathbb{C}$ by $\psi_{p}\left(\lambda_{p}\right)=\lambda_{p}$ for all $p \in P$ and $\lambda_{p} \in \mathcal{E}_{p}$. So $\psi_{e}$ is faithful. Let $1_{a} \in \mathbb{K}\left(\mathcal{E}_{a}\right)$. Then $\psi_{e}(1)-\psi^{(a)}\left(1_{a}\right)=0$ but $\widetilde{\iota}_{e}^{p}(1)-\widetilde{\iota}_{a}^{p}\left(1_{a}\right) \neq 0$ for all $p \geq b$. Hence the image of $1-1_{a}$ in $\mathcal{N} \mathcal{O}_{\mathcal{E}}$ is nonzero and it becomes clear that $\mathcal{N O} \mathcal{O}_{\mathcal{E}}$ and $A \times_{\mathcal{E}} P$ are not isomorphic, since $j_{e}(1)-j^{(a)}\left(1_{a}\right)=0$ in the latter. For this example, $A \times_{\mathcal{E}} P$ is the universal $\mathrm{C}^{*}$-algebra generated by a unitary. That is, $A \times_{\mathcal{E}} P \cong C(\mathbb{T})$ with $j_{a}\left(\lambda_{a}\right)=\lambda_{a} z$ and $j_{e}(\lambda)=\lambda$, where $z: \mathbb{T} \rightarrow \mathbb{C}$ is the inclusion function. 
Proposition 6.3.6. Let $(G, P)$ be a quasi-lattice ordered group and let $\mathcal{E}=\left(\mathcal{E}_{p}\right)_{p \in P}$ be a compactly aligned product system over $P$. Suppose either that $\mathcal{E}$ is faithful or that $P$ is directed and the representation of $\mathcal{E}$ in $\mathcal{N O}_{\mathcal{E}}$ is injective. Then $\mathcal{N O}_{\mathcal{E}}$ and $A \times_{\mathcal{E}} P$ are canonically isomorphic to each other.

Proof. Let $\bar{j}_{\mathcal{E}}$ denote the representation of $\mathcal{E}$ in $\mathcal{N} \mathcal{O}_{\mathcal{E}}$. By Proposition 6.3.4 ker $\bar{j}_{\mathcal{N}} \cap \mathcal{N} \mathcal{T}_{\mathcal{E}}^{e} \subseteq$ ker $q_{\mathcal{N}}$. In particular, $j_{\mathcal{E}}$ is an injective Cuntz-Nica-Pimsner covariant representation of $\mathcal{E}$ in $A \times{ }_{\mathcal{E}} P$. Hence, [15. Proposition 3.7] implies that the induced *-homomorphism $j: \mathcal{N} \mathcal{O}_{\mathcal{E}} \rightarrow A \times_{\mathcal{E}} P$ is faithful on the fixed-point algebra $\mathcal{N} \mathcal{O}_{\mathcal{E}}^{e}$. Therefore, $\bar{j}_{\mathcal{N}}$ vanishes on ker $q_{\mathcal{N}}$ and it factors through $A \times_{\mathcal{E}} P$. Thus $\widehat{\bar{j}}_{\mathcal{N}}$ is the inverse of $j$

\subsubsection{Cuntz-Pimsner algebras}

Recall that Fowler's Cuntz-Pimsner algebra of a product system $\mathcal{E}=\left(\mathcal{E}_{p}\right)_{p \in P}$, denoted by $\mathcal{O}_{\mathcal{E}}$, is the universal $\mathrm{C}^{*}$-algebra for representations that are Cuntz-Pimsner covariant on $J_{p}:=\varphi^{-1}\left(\mathbb{K}\left(\mathcal{E}_{p}\right)\right)[26$. Proposition 2.9]. See also Remark 4.2.8 Our next result provides sufficient conditions for $A \times{ }_{\mathcal{E}} P$ to coincide with Fowler's Cuntz-Pimsner algebra if $P$ is a cancellative right Ore monoid, that is, $P$ is cancellative and $p P \cap q P \neq \emptyset$ for all $p, q \in P$. In this case, $P$ can be embedded in a group $G$ so that $G=P P^{-1}$.

Proposition 6.3.7. Let $P$ be a cancellative right Ore monoid and let $\mathcal{E}=\left(\mathcal{E}_{p}\right)_{p \in P}$ be a product system that is faithful and proper. Then $A \times_{\mathcal{E}} P$ is canonically isomorphic to Fowler's Cuntz-Pimsner algebra.

Proof. Observe that $J_{p}=A$ for all $p \in P$. We begin by verifying that the representation of $\mathcal{E}$ in $A \times \mathcal{E} P$ is Cuntz-Pimsner covariant on $A$ for each $p$ in $P$. Indeed, set $F:=\{p\}$. Since $\mathcal{E}$ is faithful, it follows that $I_{r^{-1}(r \vee p)}=\{0\}$ if $r \notin p P$. Hence

$$
\mathcal{E}_{\{p\}}=\bigoplus_{r \in p P} \mathcal{E}_{r} .
$$

Hence $j_{\mathcal{E}}$ is Cuntz-Pimsner covariant on $J_{p}$ for each $p \in P$. We then obtain a ${ }^{*}$-homomorphism $j: \mathcal{O}_{\mathcal{J}, \mathcal{E}} \rightarrow A \times \times_{\mathcal{E}} P$.

By 5 . Theorem 3.16], we may view $\mathcal{O}_{\mathcal{J}, \mathcal{E}}^{e}$ as the inductive limit of $\left(\mathbb{K}\left(\mathcal{E}_{p}\right)\right)_{p \in P}$. Thus $j$ is faithful on $\mathcal{O}_{\mathcal{J}, \mathcal{E}}^{e}$ since it is so on all of the $\mathbb{K}\left(\mathcal{E}_{p}\right)$ 's. The quotient map $q: \mathcal{T}_{\mathcal{E}} \rightarrow A \times_{\mathcal{E}} P$ is the composition of $j$ with the quotient map from $\mathcal{T}_{\mathcal{E}}$ to $\mathcal{O}_{\mathcal{J}, \mathcal{E}}$. Hence the representation of $\mathcal{E}$ in this latter $\mathrm{C}^{*}$-algebra must vanish on $J_{\infty}$. The induced ${ }^{*}$-homomorphism $A \times_{\mathcal{E}} P \rightarrow \mathcal{O}_{\mathcal{J}, \mathcal{E}}$ is then the inverse of $j$.

Proposition 6.3.8. Let $(G, P)$ be a quasi-lattice ordered group and let $\mathcal{E}=\left(\mathcal{E}_{p}\right)_{p \in P}$ be a simplifiable product system of Hilbert bimodules as in Definition 5.1.5. Then $A \times_{\mathcal{E}} P$ is canonically isomorphic to $\mathcal{O}_{\mathcal{I}_{\mathcal{E}}, \mathcal{E}}$, where $\mathcal{I}_{\mathcal{E}}$ is the family of Katsura's ideals for $\mathcal{E}$.

Proof. We begin by proving that the canonical representation of $\mathcal{E}$ in $A \times{ }_{\mathcal{E}} P$ factors through $\mathcal{O}_{\mathcal{I}_{\mathcal{E}}, \mathcal{E}}$. Let $p \in P$ and let $s \in P$ be such that $r \geq p$. Axiom (ii) of Definition 5.1.5 entails $\varphi_{r}\left(\left\langle\left\langle\mathcal{E}_{p} \mid \mathcal{E}_{p}\right\rangle\right\rangle\right) \mathcal{E}_{r}=\{0\}$ if $p \vee r=\infty$. Suppose that $p \vee r<\infty$. Then

$$
\left\langle\left\langle\mathcal{E}_{p} \mid \mathcal{E}_{p}\right\rangle\right\rangle\left\langle\left\langle\mathcal{E}_{r} \mid \mathcal{E}_{r}\right\rangle\right\rangle \subseteq\left\langle\left\langle\mathcal{E}_{p \vee r} \mid \mathcal{E}_{p \vee r}\right\rangle\right\rangle
$$

In particular,

$$
\left\langle\left\langle\mathcal{E}_{p} \mid \mathcal{E}_{p}\right\rangle\right\rangle\left\langle\left\langle\mathcal{E}_{r} \mid \mathcal{E}_{r} \operatorname{ker} \varphi_{r^{-1}(p \vee r)}\right\rangle\right\rangle=\{0\}
$$

because $\left\langle\left\langle\mathcal{E}_{p \vee r} \mid \mathcal{E}_{p \vee r}\right\rangle\right\rangle$ acts faithfully on $\mathcal{E}_{p \vee r}$. Hence $\varphi_{r}\left(\left\langle\left\langle\mathcal{E}_{p} \mid \mathcal{E}_{p}\right\rangle\right\rangle\right) \mathcal{E}_{r} \operatorname{ker} \varphi_{r^{-1}(p \vee r)}=\{0\}$. So given $a$ in $\left\langle\left\langle\mathcal{E}_{p} \mid \mathcal{E}_{p}\right\rangle\right\rangle$, take $F=\{p\}$. Then $t_{F}^{e}(a)-t_{F}^{(p)}\left(\varphi_{p}(a)\right)=0$ on $\mathcal{E}_{F}$. By Proposition 6.3.2.

$$
j^{(p)}\left(\varphi_{p}(a)\right)=j_{e}(a) .
$$

This shows that $j=\left\{j_{p}\right\}_{p \in P}$ is an injective representation of $\mathcal{E}$ in $A \times{ }_{\mathcal{E}} P$ that is Cuntz-Pimsner covariant on $I_{\mathcal{E}_{p}}=\left\langle\left\langle\mathcal{E}_{p} \mid \mathcal{E}_{p}\right\rangle\right\rangle$ for all $p \in P$. So it induces a ${ }^{*}$-homomorphism $\phi: \mathcal{O}_{\mathcal{I}_{\mathcal{E}} \mathcal{E}} \rightarrow A \times_{\mathcal{E}} P$. Since $\phi$ is a surjective grading-preserving ${ }^{*}$-homomorphism and $\mathcal{O}_{\mathcal{I}_{\mathcal{E}}, \mathcal{E}}^{e} \cong A$, the universal property of $A \times_{\mathcal{E}} P$ tells us that $\phi$ is an isomorphism. 


\subsubsection{Semigroup $C^{*}$-algebras}

The semigroup $\mathrm{C}^{*}$-algebra as introduced by Murphy in $\left[44\right.$ is the universal $\mathrm{C}^{*}$-algebra for representations of $P$ by isometries, also called isometric representations. Unlike the group case, the resulting $\mathrm{C}^{*}$-algebra is usually badly behaved. For instance, it is not nuclear even when the semigroup in question is $\mathbb{N} \times \mathbb{N}$ (see 45$]$ ). For semigroups that are positive cones of quasi-lattice ordered groups, Nica considered in 47 a sub-class of isometric representations, namely, those satisfying the Nica covariance condition (see Example 4.2.13. In this setting, he also introduced a notion of amenability for a quasi-lattice ordered group $(G, P)$ and proved, for instance, that $\left(\mathbb{F}_{n}, \mathbb{F}_{n}^{+}\right)$is amenable. Xin Li realised that one should also take into account the family of right ideals of $P$ and proposed a construction generalising that of Nica to left cancellative semigroups [39]. In analogy with the group case, he was able to relate amenability of a semigroup to its associated $\mathrm{C}^{*}$-algebra (see 39 , Section 4]). In this subsection, we study the relationship between covariance algebras and the semigroup $\mathrm{C}^{*}$-algebras of Xin Li. Under a certain assumption involving the family of constructible right ideals of $P$, we will show that we can recover the semigroup $\mathrm{C}^{*}$-algebra of Xin Li from the covariance algebra of a certain product system. This is obtained in [5, Section 5] for Ore monoids.

Let us first recall Li's construction. Assume that $G$ is generated by $P$. Given $\alpha=\left(p_{1}, p_{2}, \ldots, p_{2 k}\right) \subseteq$ $P$, define

$$
F_{\alpha}=\left\{p_{2 k}^{-1} p_{2 k-1}, p_{2 k}^{-1} p_{2 k-1} p_{2 k-2}^{-1} p_{2 k-3}, \ldots, p_{2 k}^{-1} p_{2 k-1} p_{2 k-2}^{-1} \cdots p_{2}^{-1} p_{1}\right\} .
$$

Then $K_{\left\{F_{\alpha}, e\right\}}$ is a right ideal in $P$. This corresponds to the right ideal

$$
p_{2 k}^{-1} p_{2 k-1} p_{2 k-2}^{-1} \cdots p_{2}^{-1} p_{1} P
$$

in the notation of [39]. Given words $\alpha_{1}, \alpha_{2}, \ldots, \alpha_{n}$ in $P$, the intersection

$$
\bigcap_{i=1}^{n} K_{\left\{F_{\alpha_{i}}, e\right\}}
$$

is again a right ideal in $P$. Let $\mathcal{J}$ be the smallest family of right ideals of $P$ containing the "constructible" right ideals as above and the empty set $\emptyset$. This is closed under finite intersection. In addition, if $S \in \mathcal{J}$, then $p S \in \mathcal{J}$ and $p^{-1} S \in \mathcal{J}$, where $p S$ and $p^{-1} S$ denote the image and pre-image of $S$, respectively, under left multiplication by $p$. The following is [39, Definition 3.2].

Definition 6.3.10. Let $P$ be a subsemigroup of a group $G$. The semigroup $\mathrm{C}^{*}$-algebra of $P$, denoted by $\mathrm{C}_{s}^{*}(P)$, is the universal $\mathrm{C}^{*}$-algebra generated by a family of isometries $\left\{v_{p} \mid p \in P\right\}$ and projections $\left\{e_{S} \mid S \in \mathcal{J}\right\}$ satisfying the following:

(i) $v_{p} v_{q}=v_{p q}$,

(ii) $e_{\emptyset}=0$,

(iii) $v_{p_{1}}^{*} v_{p_{2}} \cdots v_{p_{2 k-2}} v_{p_{2 k-1}}^{*} v_{p_{2 k}}=e_{K_{\left\{F_{\alpha}, e\right\}}}$ whenever $\alpha=\left(p_{1}, p_{2}, \ldots, p_{2 k}\right)$ is a word in $P$ with $p_{1}^{-1} p_{2} \cdots p_{2 k-2} p_{2 k-1}^{-1} p_{2 k}=$ $e$ in $G$.

The family $\mathcal{J}$ of right ideals of $P$ is called independent (see [39, Definition 2.26]) if given a right ideal of $P$ of the form

$$
S=\bigcup_{i=1}^{m} S_{i},
$$

with $S_{i} \in \mathcal{J}$ for all $i \in\{1, \ldots, m\}$, then $S=S_{i}$ for some $i \in\{1, \ldots, m\}$. By [39, Lemma 3.3], $e_{S_{1}} e_{S_{2}}=e_{S_{1} \cap S_{2}}$ in $\mathrm{C}_{s}^{*}(P)$ for all $S_{1}, S_{2}$ in $\mathcal{J}$ and hence the closed linear span of the projections $\left\{e_{S} \mid S \in \mathcal{J}\right\}$ is a commutative $\mathrm{C}^{*}$-subalgebra of $\mathrm{C}_{s}^{*}(P)$. If $\mathcal{J}$ is independent, this $\mathrm{C}^{*}$-subalgebra is canonically isomorphic to the $\mathrm{C}^{*}$-subalgebra of $\ell^{\infty}(P)$ generated by the characteristic functions on elements of $\mathcal{J}$ [39, Corollary 3.4]. Let us denote this latter $\mathrm{C}^{*}$-algebra by $A$. That is,

$$
A=\overline{\operatorname{span}}\left\{\chi_{S} \mid S \in \mathcal{J}\right\}
$$

where $\chi_{S} \in \ell^{\infty}(P)$ is the characteristic function on $S$. This will be the coefficient algebra of our product system $\mathcal{E}$. The idea is taken from [5, Section 5]. Our assumption, however, is different: we require $P$ to be embeddable in a group, as usual. So we follow [39, Definition 3.2]. 


\section{6. $\mathrm{C}^{*}$-ALGEBRAS FOR PRODUCT SYSTEMS OVER SUBSEMIGROUPS OF GROUPS}

There is a semigroup action $\beta: P \rightarrow \operatorname{End}(A)$ by injective endomorphisms with hereditary range as follows. Let $\beta_{p}$ be defined by $\chi_{S} \mapsto \chi_{p S}$. Its range $\beta_{p}(A)$ is the corner $\chi_{p P} A \chi_{p P}$ and hence it is hereditary. This gives us a product system over $P$ as in Example 4.2.10 The correspondence $\mathcal{E}_{p}: A \leadsto A$ is $A \chi_{p P}$ with the following structure: we use the inverse $\beta_{p}^{-1}$ to define the $A$-valued inner product, so that

$$
\left\langle a \chi_{p P} \mid b \chi_{p P}\right\rangle:=\beta_{p}^{-1}\left(\chi_{p P} a^{*} \cdot b \chi_{p P}\right) .
$$

In particular, $\left\langle\chi_{S} \chi_{p P} \mid \chi_{p P}\right\rangle=\chi_{\left(p^{-1} S\right) \cap P}$ for all $S \in \mathcal{J}$. The right action of $A$ on $\mathcal{E}$ is implemented by $\beta_{p}$. That is, $\left(b \chi_{p P}\right) \cdot \chi_{S}=b \chi_{p S}$. The left action is then defined by left multiplication $a \cdot\left(b \chi_{p P}\right)=$ $a b \chi_{p P}$. Finally, the isomorphism $\mu_{p, q}: \mathcal{E}_{p} \otimes_{A} \mathcal{E}_{q} \cong \mathcal{E}_{p q}$ sends $a \chi_{p P} \otimes_{A} b \chi_{q P}$ to $a \chi_{p P} \beta_{p}(b) \chi_{p q P}$. As in Example $4.2 .10 \mathcal{E}_{p}$ is a Hilbert bimodule with left $A$-valued inner product given by

$$
\left\langle\left\langle a \chi_{p P} \mid b \chi_{p P}\right\rangle\right\rangle=a \chi_{p P} b^{*} .
$$

In particular, this is simplifiable if $(G, P)$ is a quasi-lattice ordered group.

Proposition 6.3.11. Suppose that $\mathcal{J}$ is independent. The semigroup $\mathrm{C}^{*}$-algebra $\mathrm{C}_{s}^{*}(P)$ is naturally isomorphic to $A \times_{\mathcal{E}} P$.

Proof. Let us define a *-homomorphism $\mathrm{C}_{s}^{*}(P) \rightarrow A \times_{\mathcal{E}} P$ by using the universal property of $\mathrm{C}_{s}^{*}(P)$. For each $p \in P$, put $u_{p}:=j_{p}\left(\chi_{p P}\right)$. Thus $u$ is an isometric representation of $P$ in $A \times_{\mathcal{E}} P$. Given $S \in \mathcal{J}$, set $\bar{e}_{S}=\chi_{S}$. In order to prove that this data also satisfies the condition (iii) of Definition 6.3.10, let $\alpha=\left(p_{1}, p_{2}, \ldots, p_{2 k}\right)$ be a word in $P$ with $p_{1}^{-1} p_{2} \cdots p_{2 k-2} p_{2 k-1}^{-1} p_{2 k}=e$. Let $F_{\alpha}$ be as in 6.3 .9$)$. Let us show that

$$
t_{F_{\alpha}}\left(\widetilde{t}_{p_{1}}\left(\chi_{p_{1} P}\right)^{*} \widetilde{t}_{p_{2}}\left(\chi_{p_{2} P}\right) \cdots \widetilde{t}_{p_{2 k-1}}\left(\chi_{p_{2 k-1} P}\right)^{*} \widetilde{t}_{p_{2 k}}\left(\chi_{p_{2 k-1} P}\right)-\widetilde{t}\left(\chi_{K_{\left\{F_{\alpha}, e\right\}}}\right)\right)=0
$$

on $\mathcal{E}_{F_{\alpha}}$. This is clearly true if $K_{\left\{F_{\alpha}, e\right\}}=\emptyset$ or $K_{\left\{F_{\alpha}, e\right\}}=P$. So let us assume otherwise. The ideal $I_{e \vee F_{\alpha}} \triangleleft A$ is generated by the characteristic functions on the right ideals that have empty intersection with

$$
K_{\left\{F_{\alpha}, e\right\}}=p_{2 k}^{-1} p_{2 k-1} p_{2 k-2}^{-1} \cdots p_{2}^{-1} p_{1} P
$$

so that $\chi_{K_{\left\{F_{\alpha}, e\right\}}} I_{e \vee F_{\alpha}}=0$. Similarly, let $r \notin K_{\left\{F_{\alpha}, e\right\}}$. Observe that $\chi_{K_{\left\{F_{\alpha}, e\right\}}}$ vanishes on $\mathcal{E}_{r}$ whenever $r P \cap K_{\left\{F_{\alpha}, e\right\}}=\emptyset$. If $r P \cap K_{\left\{F_{\alpha}, e\right\}} \neq \emptyset$, then $I_{r^{-1}\left(r \vee F_{\alpha}\right)}$ consists of those functions in $A$ that vanish on $P \cap r^{-1} K_{\left\{F_{\alpha}, e\right\}}$. In particular,

$$
\begin{aligned}
\varphi_{r}\left(\chi_{\left.K_{\left\{F_{\alpha}, e\right\}}\right)\left(\chi_{r P}\right) \cdot I_{r^{-1}\left(r \vee F_{\alpha}\right)}}=\chi_{K_{\left\{F_{\alpha}, e\right\}} \cap r P} \beta_{r}\left(I_{r^{-1}\left(r \vee F_{\alpha}\right)}\right)\right. \\
=\chi_{K_{\left\{F_{\alpha}, e\right\}} \cap t P} I_{\left(t \vee F_{\alpha}\right)}=\{0\} .
\end{aligned}
$$

For $r \in K_{\left\{F_{\alpha}, e\right\}}$, one may easily verify that the left-hand side of 6.3 .12 also vanishes on $\mathcal{E}_{r}$. This proves our claim that the condition (iii) of Definition 6.3.10 is satisfied. So we obtain a *-homomorphism $\phi: \mathrm{C}_{s}^{*}(P) \rightarrow A \times{ }_{\mathcal{E}} P$. This sends $v_{p}$ to the isometry $u_{p}$ and $e_{S}$ to $j_{e}\left(\chi_{S}\right)$.

In order to define a representation of $\mathcal{E}$ in $\mathrm{C}_{s}^{*}(P)$, we invoke the assumption that $\mathcal{J}$ is independent. As mentioned before the statement, in this case the commutative $\mathrm{C}^{*}$-subalgebra of $\mathrm{C}_{s}^{*}(P)$ generated by the projections $\left\{e_{S} \mid S \in \mathcal{J}\right\}$ is canonically isomorphic to $A$. So there is a ${ }^{*}$-homomorphism $A \rightarrow \mathrm{C}_{s}^{*}(P)$ which maps $\chi_{S}$ to $e_{S}$. Lemmas 2.8 and 3.3 of [39] imply the relations

$$
v_{p} e_{S} v_{p}^{*}=e_{p S}, \quad v_{p}^{*} e_{S} v_{p}=e_{p^{-1} S \cap P}
$$

in $\mathrm{C}_{s}^{*}(P)$ for all $p \in P$ and $S \in \mathcal{J}$. Hence the map which sends $\chi_{p P} \in \mathcal{E}_{p}$ to the isometry $v_{p}$ together with the ${ }^{*}$-homomorphism $\chi_{S} \mapsto e_{S}$ gives us a representation of $\mathcal{E}$ in $\mathrm{C}_{s}^{*}(P)$. The induced *-homomorphism $\mathcal{T}_{\mathcal{E}} \rightarrow \mathrm{C}_{s}^{*}(P)$ preserves the $G$-grading for the coaction of $G$. Moreover, it follows from the condition (iii) and the equality $v_{e}=1$ that the fixed-point algebra $\mathrm{C}_{s}^{*}(P)^{e}$ for such a coaction is the $\mathrm{C}^{*}$-algebra generated by the projections $\left\{e_{S} \mid S \in \mathcal{J}\right\}$, which in turn is isomorphic to $A$. Hence $\phi$ is injective on $\mathrm{C}_{s}^{*}(P)^{e}$. By the same argument employed in the proof of Proposition 6.3.7 we conclude that $\phi$ is an isomorphism.

The proof of the previous proposition shows that, in general, $A \times{ }_{\mathcal{E}} P$ is a quotient of $\mathrm{C}_{s}^{*}(P)$. It is 
isomorphic to the $\mathrm{C}^{*}$-algebra $\mathrm{C}_{s}^{*}(\mathrm{\cup})(P)$ in the notation of 39 . Indeed, let

$$
\mathcal{J}^{\cup}:=\left\{\bigcup_{i=1}^{m} S_{i} \mid S_{i} \in \mathcal{J}\right\} .
$$

Let $\mathrm{C}_{s}^{*(\cup)}(P)$ be the universal $\mathrm{C}^{*}$-algebra generated by isometries $\left\{v_{p} \mid p \in P\right\}$ and projections $\left\{e_{S} \mid S \in\right.$ $\left.\mathcal{J}^{\cup}\right\}$ satisfying the conditions (i)-(iii) of Definition 6.3.10 with the additional relation

(iv) $e_{S_{1} \cup S_{2}}=e_{S_{1}}+e_{S_{2}}-e_{S_{1} \cap S_{2}}$ for all $S_{1}, S_{2} \in \mathcal{J}^{\cup}$.

The $\mathrm{C}^{*}$-algebra $\mathrm{C}_{s}^{*(\cup)}(P)$ coincides with $\mathrm{C}_{s}^{*}(P)$ whenever $\mathcal{J}$ is independent (see 39 , Proposition 2.24]). The next result generalises Proposition 6.3.11

Corollary 6.3.13. The semigroup $\mathrm{C}^{*}$-algebra $\mathrm{C}_{s}^{*(\cup)}(P)$ is naturally isomorphic to $A \times{ }_{\mathcal{E}} P$.

Proof. It follows from [39, Lemma 3.3] and [39, Corollary 2.22] that the $\mathrm{C}^{*}$-subalgebra of $\mathrm{C}_{s}^{*(\cup)}(P)$ generated by the $e_{S}$ 's is naturally isomorphic to $A$. Again condition (iii) of Definition 6.3.10 implies that such a $\mathrm{C}^{*}$-subalgebra coincides with the fixed-point algebra for the canonical coaction of $G$ on $\mathrm{C}_{s}^{*(\cup)}(P)$. Now we may employ the same argument used in the proof of Proposition 6.3.11 to obtain an isomorphism $\mathrm{C}_{s}^{*(\cup)}(P) \cong A \times_{\mathcal{E}} P$.

\subsubsection{Crossed products by interaction groups}

In this subsection, we will show how Exel's crossed products by interaction groups fit into our approach. This notion of crossed products was introduced in $[22$ in order to study semigroups of unital and injective endomorphisms. We first recall some concepts from his work, although many details will be omitted. An interaction group is a triple $(A, G, V)$, where $A$ is a unital $\mathrm{C}^{*}$-algebra, $G$ is a group and $V$ is a partial representation of $G$ in the Banach algebra of bounded operators on $A$. This consists of a family $\left\{V_{g}\right\}_{g \in G}$ of continuous operators on $A$ with $V_{1}=\operatorname{id}_{A}$ and

$$
V_{g} V_{h} V_{h^{-1}}=V_{g h} V_{h^{-1}}, \quad V_{g^{-1}} V_{g} V_{h}=V_{g^{-1}} V_{g h}
$$

for all $g, h \in G$. It follows that $E_{g}:=V_{g} V_{g^{-1}}$ is an idempotent for each $g \in G$ and $E_{g} E_{h}=E_{h} E_{g}$, $g, h \in G$. The partial representation is also assumed to satisfy the following conditions:

(i) $V_{g}$ is a positive map,

(ii) $V_{g}(1)=1$,

(iii) $V_{g}(a b)=V_{g}(a) V_{g}(b)$ if $a$ or $b$ belong to the range of $V_{g^{-1}}$.

For all $g \in G$, the idempotent $E_{g}$ is a conditional expectation onto the range of $V_{g}$. An interaction group is said to be nondegenerate if $E_{g}$ is faithful for all $g$ in $G$. That is, $E_{g}\left(a^{*} a\right)=0$ implies $a=0$ (see [22, Definition 3.3]).

Frow now on let us fix a nondegenerate interaction group $(A, G, V)$. Given a unital $\mathrm{C}^{*}$-algebra $B$, recall that $v: G \rightarrow B$ is a ${ }^{*}$-partial representation if it is a partial representation satisfying $v_{g}^{*}=v_{g^{-1}}$ for all $g \in G$. A covariant representation of $(A, G, V)$ in $B$ is a pair $(\pi, v)$, where $\pi: A \rightarrow B$ is a unital *-homomorphism and $v$ is a ${ }^{*}$-partial representation of $G$ in $B$ such that

$$
v_{g} \pi(a) v_{g^{-1}}=\pi\left(V_{g}(a)\right) v_{g} v_{g^{-1}} .
$$

The Toeplitz algebra of $(A, G, V)$, denoted by $\mathcal{T}(A, G, V)$, is the universal $\mathrm{C}^{*}$-algebra for covariant representations of $(A, G, V)$. It is generated by a copy of $A$ and elements $\left\{\widehat{s}_{g}\right\}_{g \in G}$ so that $\widehat{s}: g \mapsto \widehat{s}_{g}$ is a ${ }^{*}$-partial representation and the pair $\left(j_{V}, \widehat{s}\right)$ is a covariant representation of $(A, G, V)$ in $\mathcal{T}(A, G, V)$, where $j_{V}: A \rightarrow \mathcal{T}(A, G, V)$ denotes the canonical embedding.

In order to recall the notion of redundancy introduced by Exel in [22], let us first define certain subspaces of $\mathcal{T}(A, G, V)$. Given a word $\alpha=\left(g_{1}, g_{2}, \ldots, g_{n}\right)$ in $G$, set

$$
\widehat{s}_{\alpha}=\widehat{s}_{g_{1}} \widehat{s}_{g_{2}} \cdots \widehat{s}_{g_{n}} .
$$


Let $\widehat{\mathcal{M}}_{\alpha}=j_{V}(A) \widehat{s}_{\alpha} j_{V}(A)$ and $e_{\alpha}:=\widehat{s}_{\alpha} \widehat{s}_{\alpha^{-1}}$, where $\alpha^{-1}=\left(g_{n}^{-1}, \cdots, g_{2}^{-1}, g_{1}^{-1}\right)$. Then $\widehat{s}_{\alpha} j_{V}(a) \widehat{s}_{\alpha^{-1}}=$ $j_{V}\left(V_{\alpha}(a)\right) e_{\alpha}$ and, by [22, Proposition 2.7], $e_{\alpha}$ is also an idempotent. The subspace $\widehat{\mathcal{Z}}_{\alpha}$ associated to the word $\alpha$ will be the closed linear span of elements of the form

$$
j_{V}\left(a_{0}\right) \widehat{s}_{g_{1}} j_{V}\left(a_{1}\right) \widehat{s}_{g_{2}} \cdots \widehat{s}_{g_{n}} j_{V}\left(a_{n}\right)
$$

with $a_{0}, a_{1}, a_{2}, \ldots, a_{n} \in A$. We set $\widehat{\mathcal{Z}}_{\alpha}=j_{V}(A)$ in case $\alpha$ is the empty word. Observe that we always have $\widehat{\mathcal{M}}_{\alpha} \subseteq \widehat{\mathcal{Z}}_{\alpha}$. We also associate a finite subset of $G$ to the word $\alpha$ by letting

$$
\mu(\alpha)=\left\{e, g_{1}, g_{1} g_{2}, \ldots, g_{1} g_{2} \cdots g_{n}\right\},
$$

so that $\mu(\alpha)=\{e\}$ if $\alpha$ is the empty word. We further let $\dot{\alpha}=g_{1} g_{2} \cdots g_{n}$. If $\dot{\alpha}=e$, it follows that $\mu(\alpha)=\mu\left(\alpha^{-1}\right)$. We denote by $\mathcal{W}_{\alpha}$ the set of all words $\beta$ in $G$ with $\mu(\beta) \subseteq \mu(\alpha)$ and $\dot{\beta}=e$ and let

$$
\widehat{\mathcal{Z}}^{\mu(\alpha)}:=\overline{\operatorname{span}}\left\{c_{\beta} \mid c_{\beta} \in \widehat{\mathcal{Z}}_{\beta}, \beta \in \mathcal{W}_{\alpha}\right\} .
$$

This is a $\mathrm{C}^{*}$-subalgebra of $\mathcal{T}(A, G, V)$ since $\beta \in \mathcal{W}_{\alpha}$ if and only if $\beta^{-1} \in \mathcal{W}_{\alpha}$ and $\mathcal{W}_{\alpha}$ is also closed under concatenation of words (see 22 , Proposition 4.7] for further details). In addition, $\widehat{\mathcal{Z}}^{\mu(\alpha)} \widehat{\mathcal{M}}_{\alpha} \subseteq \widehat{\mathcal{M}}_{\alpha}$.

Definition 6.3.14. Let $\alpha$ be a word in $G$. We say that $c \in \widehat{\mathcal{Z}}^{\mu(\alpha)}$ is an $\alpha$-redundancy if $c \widehat{\mathcal{M}}_{\alpha}=\{0\}$.

The crossed product of $A$ by $G$ under $V$, denoted by $A \rtimes_{G} V$, is the universal C*-algebra for covariant representations that vanish on all redundancies. Thus $A \rtimes_{G} V$ is isomorphic to the quotient of $\mathcal{T}(A, G, V)$ by the ideal generated by all redundancies. A covariant representation of $(A, G, V)$ that vanishes on such an ideal was called strongly covariant by Exel. He was able to prove that $A$ is embedded into $A \rtimes_{G} V$. The crossed product carries a canonical $G$-grading, and a representation of $A \rtimes_{G} V$ is faithful on its fixed-point algebra if and only if it is faithful on $A$.

If $P$ is a subsemigroup of $G$, sometimes an action of $P$ on a $C^{*}$-algebra $A$ may be enriched to an interaction group $(A, G, V)$ so that $V_{p}=\alpha_{p}$ for all $p \in P$. Under certain assumptions, $V$ is unique if it exists and $A \rtimes_{G} V$ is generated by $A$ and isometries $\left\{v_{p}\right\}_{p \in P}$ [22. Theorem 12.3]. We will see that if $P$ is reversible, in the sense that $p P \cap q P \neq \emptyset$ and $P p \cap P q \neq \emptyset$ for all $p, q \in P$, and $G=P^{-1} P=P P^{-1}$, then $A \rtimes_{G} V$ can be obtained from a covariance algebra of a certain product system if $\left\{V_{p}\right\}_{p \in P}$ generates the image of $G$ under $V$. So we will assume that $V$ is an interaction group which extends an action of $P$ by endomorphisms of $A$ and $V_{p^{-1}} \circ \alpha_{p}=\mathrm{id}_{A}$. This holds if and only if the ${ }^{*}$-partial representation of $G$ in $A \rtimes_{G} V$ restricts to an isometric representation of $P$.

Lemma 6.3.15. Let $(i, s)$ denote the representation of $(A, G, V)$ in $A \rtimes_{G} V$. Then $s_{p}$ is an isometry if and only if $V_{p^{-1}} \circ \alpha_{p}=\operatorname{id}_{A}$.

Proof. Suppose that $V_{p^{-1}} \circ \alpha_{p}=\operatorname{id}{ }_{A}$. Let us prove that $\widehat{s}_{p}^{*} \widehat{s}_{p}-1$ vanishes on $\widehat{\mathcal{M}}_{\left(p^{-1}\right)}=j_{V}(A) \widehat{s}_{p^{-1}} j_{V}(A)$. Since $\widehat{s}$ is a ${ }^{*}$-partial representation of $G$, one has that $\widehat{s}_{p^{-1}}=\widehat{s}_{p}^{*}$. Put $\beta_{1}=\left(p^{-1}, p\right)$ and $\beta_{2}=(e)$. So both $\beta_{1}$ and $\beta_{2}$ belong to $\mathcal{W}_{\left(p^{-1}\right)}$ and hence $\widehat{s}_{p}^{*} \widehat{s}_{p}-1 \in \widehat{\mathcal{Z}}^{\left\{e, p^{-1}\right\}}$. Thus all we must do is prove that

$$
\left(\widehat{s}_{p}^{*} \widehat{s}_{p}-1\right) j_{V}(A) \widehat{s}_{p}^{*} j_{V}(A)=\{0\} .
$$

To do so, let $a \in A$. Then

$$
\widehat{s}_{p}^{*} \widehat{s}_{p} j_{V}(a) \widehat{s}_{p}^{*}=\widehat{s}_{p}^{*} j_{V}\left(V_{p}(a)\right) \widehat{s}_{p} \widehat{s}_{p}^{*}=j_{V}\left(V_{p^{-1}}\left(\alpha_{p}(a)\right)\right) \widehat{s}_{p}^{*} \widehat{s}_{p} \widehat{s}_{p}^{*}=j_{V}(a) \widehat{s}_{p}^{*} .
$$

This proves that $\widehat{s}_{p}^{*} \widehat{s}_{p}-1$ is a redundancy. Hence $s_{p}$ is an isometry in $A \rtimes_{G} V$.

Now assume that $s_{p}$ is an isometry. For each $a$ in $A$,

$$
i(a)=s_{p}^{*} s_{p} i(a) s_{p}^{*} s_{p}=i\left(V_{p^{-1}}\left(\alpha_{p}(a)\right)\right) .
$$

This shows that $V_{p^{-1}} \circ \alpha_{p}=\operatorname{id}_{A}$ because $A$ is embedded into $A \rtimes_{G} V$.

Thus in order to build a product system over $P$ so that it encodes the interaction group, we suppose that $V_{p^{-1}} \circ \alpha_{p}=\operatorname{id}_{A}$ for all $p \in P$. It follows from [22, Lemma 2.3] that, for all $p, q \in P$, we have

$$
V_{q^{-1}} V_{p^{-1}}=V_{q^{-1} p^{-1}}, \quad V_{p^{-1} q}=V_{p^{-1}} V_{q} .
$$


Let us now describe the product system associated to $V$. This is defined as in Example 4.2 .10 Unlike in 38, here we do not require $P$ to be abelian since we assume $V_{p^{-1}} \circ \alpha_{p}=\mathrm{id}_{A}$. We set $\mathcal{E}_{p}:=A$, endowed with the right action of $A$ through $a \cdot b:=a \alpha_{p}(b)$ and the $A$-valued inner product $\langle a \mid b\rangle=V_{p^{-1}}\left(a^{*} b\right)$. This provides $\mathcal{E}_{p}$ with a structure of right Hilbert $A$-module because $V_{p^{-1}}\left(a^{*} a\right)=0 \Leftrightarrow a=0$ and $V_{p^{-1}}(a b)=V_{p^{-1}}(a) V_{p^{-1}}(b)$ whenever $b$ lies in the range of $\alpha_{p}$. The ${ }^{*}$-homomorphism $\varphi_{p}: A \rightarrow \mathbb{B}\left(\mathcal{E}_{p}\right)$ is given by the multiplication on $A$, so that $\varphi_{p}(a) \cdot b=a b$ for all $a \in A, b \in \mathcal{E}_{p}$. The correspondence isomorphism $\mu_{p, q}: \mathcal{E}_{p} \otimes_{A} \mathcal{E}_{q} \cong \mathcal{E}_{p q}$ sends an elementary tensor $a \otimes b$ to $a \alpha_{p}(b)$. Using that $\alpha_{p}$ is an endomorphism of $A$, we deduce that $\mu_{p, q}$ preserves the bimodule structure. It is also surjective because $\alpha_{p}$ is unital for all $p \in P$.

Lemma 6.3.16. $\mathcal{E}=\left(\mathcal{E}_{p}\right)_{p \in P}$ is a product system.

Proof. We will prove that $\mu_{p, q}$ preserves the inner product and that the multiplication in $\mathcal{E}$ is associative.

Let $a_{0}, a_{1}, b_{0}, b_{1} \in A$. Then

$$
\begin{aligned}
\left\langle a_{0} \otimes b_{0} \mid a_{1} \otimes b_{1}\right\rangle & =V_{q^{-1}}\left(b_{0}^{*} V_{p^{-1}}\left(a_{0}^{*} a_{1}\right) b_{1}\right) \\
& =V_{q^{-1}}\left(V_{p^{-1}}\left(\alpha_{p}\left(b_{0}\right)^{*}\right) V_{p^{-1}}\left(a_{0}^{*} a_{1}\right) V_{p^{-1}}\left(\alpha_{p}\left(b_{1}\right)\right)\right) \\
& =V_{q^{-1}}\left(V_{p^{-1}}\left(\alpha_{p}\left(b_{0}\right)^{*} a_{0}^{*} a_{1} \alpha_{p}\left(b_{1}\right)\right)\right) \\
& =V_{(p q)^{-1}}\left(\alpha_{p}\left(b_{0}\right)^{*} a_{0}^{*} a_{1} \alpha_{p}\left(b_{1}\right)\right) \\
& =\left\langle\mu_{p, q}\left(a_{0} \otimes b_{0}\right) \mid \mu_{p, q}\left(a_{1} \otimes b_{1}\right)\right\rangle .
\end{aligned}
$$

This completes the proof that $\mu_{p, q}$ is an isomorphism of correspondences for all $p, q \in P$. Now let $s \in P$, $a \in \mathcal{E}_{p}, b \in \mathcal{E}_{q}$ and $c \in \mathcal{E}_{s}$. Then

$$
\begin{aligned}
\left(\mu_{p q, s}\left(\mu_{p, q} \otimes 1\right)\right)(a \otimes b \otimes c) & =a \alpha_{p}(b) \alpha_{p q}(c)=a \alpha_{p}\left(b \alpha_{q}(c)\right) \\
& =\left(\mu_{p, q s}\left(1 \otimes \mu_{q, s}\right)\right)(a \otimes b \otimes c) .
\end{aligned}
$$

Lemma 6.3.17. There is a covariant representation of $(A, G, V)$ in $A \times_{\mathcal{E}} P$. It sends $g=p^{-1} q$ to $v_{g}:=j_{p}\left(1_{p}\right)^{*} j_{q}\left(1_{q}\right)$ and a to $j_{e}(a)$. Moreover, given a word $\beta=\left(g_{1}, g_{2}, \ldots, g_{n}\right)$ in $G$, the map a $\mapsto$ $j_{e}(a) v_{\beta}$ is injective, where $v_{\beta}=v_{g_{1}} v_{g_{2}} \cdots v_{g_{n}}$.

Proof. We begin by proving that $j_{p}\left(1_{p}\right)^{*} j_{q}\left(1_{q}\right)=j_{p^{\prime}}\left(1_{p^{\prime}}\right)^{*} j_{q^{\prime}}\left(1_{q^{\prime}}\right)$ for all $p, q, p^{\prime}, q^{\prime} \in P$ such that $p^{-1} q=$ $p^{\prime-1} q^{\prime}$. To do so, we use that $P$ is also left reversible. We can find $s \in P$ with $s \in(p P \cap q P) \cap\left(p^{\prime} P \cap q^{\prime} P\right)$. Since $(A, G, V)$ is nondegenerate, $\mathcal{E}$ is faithful and hence $I_{r^{-1}(r \vee s)}=\{0\}$ for all $r \in P$ such that $r \notin s P$. So

$$
\mathcal{E}_{\{s\}}=\bigoplus_{r \in s P} \mathcal{E}_{r} .
$$

Now given $r \in s P$, we write $b_{r}$ for an element in $\mathcal{E}_{r}$. We compute

$$
\begin{aligned}
t_{\{s\}}\left(\widetilde{t}\left(1_{p}\right)^{*} \widetilde{t}\left(1_{q}\right)\right)\left(b_{r}\right) & =t_{\{s\}}\left(\widetilde{t}\left(1_{p}\right)^{*}\right)\left(\alpha_{q}\left(b_{r}\right) \otimes 1\right) \\
& =V_{p^{-1}}\left(\alpha_{q}\left(b_{r}\right)\right)=V_{p^{-1} q}\left(b_{r}\right)=V_{p^{\prime-1} q^{\prime}}\left(b_{r}\right) \\
& =t_{\{s\}}\left(\widetilde{t}\left(1_{p^{\prime}}\right)^{*} \widetilde{t}\left(1_{q^{\prime}}\right)\right)\left(b_{r}\right) .
\end{aligned}
$$

Therefore, $j_{p}\left(1_{p}\right)^{*} j_{q}\left(1_{q}\right)=j_{p^{\prime}}\left(1_{p^{\prime}}\right)^{*} j_{q^{\prime}}\left(1_{q^{\prime}}\right)$ and the map $g=p^{-1} q \mapsto j_{p}\left(1_{p}\right)^{*} j_{q}\left(1_{q}\right)$ is well defined. This gives a partial representation of $G$ in $A \times{ }_{\mathcal{E}} P$ because $V$ is a partial representation. Given $g=p^{-1} q \in G$, $v_{g^{-1}}=j_{q}\left(1_{q}\right)^{*} j_{p}\left(1_{p}\right)=v_{g}^{*}$. So $g \mapsto v_{g}$ indeed defines a ${ }^{*}$-partial representation of $G$.

Let us prove that $\left(j_{e}, v\right)$ is covariant. Take $g=p^{-1} q \in G$ and $a \in A$. Again we use the assumption that $P$ is left reversible and choose $s \in p P \cap q P$. Thus it suffices to show that

$$
t_{\{s\}}\left(\widetilde{t}\left(1_{p}\right)^{*} \widetilde{t}\left(1_{q}\right) \widetilde{t}(a) \widetilde{t}\left(1_{q}\right)^{*} \widetilde{t}\left(1_{p}\right)\right)=t_{\{s\}}\left(\widetilde{t}\left(V_{g}(a)\right) \widetilde{t}\left(1_{p}\right)^{*} \widetilde{t}\left(1_{q}\right) \widetilde{t}\left(1_{q}\right)^{*} \widetilde{t}\left(1_{p}\right)\right)
$$

on $\mathcal{E}_{r}$ for $r \in s P$. Indeed, given $b_{r} \in \mathcal{E}_{r}$, one has

$$
\begin{aligned}
t_{\{s\}}\left(\widetilde{t}\left(1_{p}\right)^{*} \widetilde{t}\left(1_{q}\right) \widetilde{t}(a) \widetilde{t}\left(1_{q}\right)^{*} \widetilde{t}\left(1_{p}\right)\right)\left(b_{r}\right) & =V_{g}\left(a V_{g^{-1}}\left(b_{r}\right)\right)=V_{g}(a) V_{g}\left(V_{g^{-1}}\left(b_{r}\right)\right) \\
& =t_{\{s\}}\left(\widetilde{t}\left(V_{g}(a)\right) \widetilde{t}\left(1_{p}\right)^{*} \widetilde{t}\left(1_{q}\right) \widetilde{t}\left(1_{q}\right)^{*} \widetilde{t}\left(1_{p}\right)\right)\left(b_{r}\right),
\end{aligned}
$$

so that $\left(j_{e}, v\right)$ is a covariant representation of $(A, G, V)$. 
Let $\beta=\left(g_{1}, \ldots, g_{n}\right)$ be a word in $G$. In order to prove that the map $a \mapsto j_{e}(a) v_{\beta}$ is injective, take $s \in K_{\mu(\beta)^{-1}}$. That is,

$$
s \in P \cap g_{n}^{-1} P \cap\left(g_{n}^{-1} g_{n-1}^{-1}\right) P \cdots \cap\left(g_{n}^{-1} g_{n-1}^{-1} \cdots g_{1}^{-1}\right) P .
$$

It exists because $G=P P^{-1}$. Using that $V_{g}$ is unital for all $g \in G$, we deduce that

$$
j_{e}(a) v_{\beta} v_{s}=j_{e}(a) v_{\beta} j_{s}(1)=j_{e}(a) j_{\dot{\beta} s}\left(V_{\beta}(1)\right)=j_{e}(a) j_{\dot{\beta} s}(1)=j_{\dot{\beta} s}(a) .
$$

Since the representation of $\mathcal{E}$ in $A \times_{\mathcal{E}} P$ is injective, the right-hand side above is nonzero. This guarantees that $a \mapsto j_{e}(a) v_{\beta}$ is an injective map.

The following is the main result of this subsection.

Proposition 6.3.18. Let $P$ be a subsemigroup of a group $G$ with $G=P^{-1} P=P P^{-1}$. Let $(A, G, V)$ be a nondegenerate interaction group extending an action $\alpha: P \rightarrow \operatorname{End}(A)$ by unital and injective endomorphisms. Suppose, in addition, that $V_{p^{-1}} \circ \alpha_{p}=\mathrm{id}_{A}$ for all $p \in P$. Then $A \rtimes_{G} V$ is isomorphic to $A \times{ }_{\mathcal{E}} P$, where $\mathcal{E}$ is the product system constructed out of $V$.

Proof. We begin by proving that $\left(j_{e}, v\right)$ factors through $A \rtimes_{G} V$. The pair $\left(j_{e}, v\right)$ induces a ${ }^{*}$-homomorphism $\widehat{\phi}: \mathcal{T}(A, G, V) \rightarrow A \times{ }_{\mathcal{E}} P$. Lemma 6.3.17 says that the map $a \mapsto j_{e}(a) v_{\beta}$ is injective for each word $\beta$ in $G$. Hence 22 , Proposition 10.5] implies that $\widehat{\phi}$ is injective on $\widehat{\mathcal{M}}_{\alpha}$. In particular, if $c \in \widehat{\mathcal{Z}}^{\mu(\alpha)}$ is an $\alpha$-redundancy, $\widehat{\phi}(c) j_{r}\left(\mathcal{E}_{r}\right)=\{0\}$ for all $r \in K_{\mu(\alpha)}$ because

$$
j_{r}(a)=j_{e}(a) j_{r}(1)=j_{e}(a) v_{\alpha} j_{\dot{\alpha}^{-1} r}(1) \in \widehat{\phi}\left(\widehat{M}_{\alpha}\right) j_{\dot{\alpha}^{-1}{ }_{r}}(1)
$$

for all $a$ in $A$. So $\widehat{\phi}(c)$ must be zero in $A \times_{\mathcal{E}} P$. This induces a *-homomorphism $\phi: A \rtimes_{G} V \rightarrow A \times_{\mathcal{E}} P$ that is faithful on $A$ and preserves the $G$-grading of $A \rtimes_{G} V$. Proposition 4.6 of [22] says that $\phi$ is also faithful on the fixed-point algebra of $A \rtimes_{G} V$. Now by Lemma 6.3.15, $s_{p}$ is an isometry in $A \rtimes_{G} V$ for all $p \in P$. Moreover, 22, Lemma 2.3] says that $s_{p} s_{q}=s_{p q}$ for all $p, q \in P$. Hence one can show that the maps $\mathcal{E}_{p} \ni 1_{p} \mapsto s_{p}$ and $a \mapsto i(a)$ give rise to a representation of $\mathcal{E}$. By applying the injectivity of $\phi$ on the fibres and the usual argument that the induced ${ }^{*}$-homomorphism $\mathcal{T}_{\mathcal{E}} \rightarrow A \rtimes_{G} V$ preserves the $G$-grading, we conclude that such a representation must factor through $A \times_{\mathcal{E}} P$. The resulting *-homomorphism is the inverse of $\phi$.

Remark 6.3.19. Let $P$ be a reversible cancellative semigroup and let $G$ be its enveloping group. Let $A$ be a unital $\mathrm{C}^{*}$-algebra and let $\alpha: P \rightarrow \operatorname{End}(A)$ be an action by injective endomorphisms. Given a not-necessarily nondegenerate interaction group $(A, G, V)$ extending $\alpha$ with $V_{p^{-1}} \circ \alpha_{p}=\mathrm{id}_{A}$, the equality $V_{q^{-1}} V_{p^{-1}}=V_{q^{-1} p^{-1}}$ still holds by [22, Lemma 2.3]. Hence one may build a product system as above by letting $\mathcal{E}_{p}:=A \alpha_{p}(1)$ and $\mu_{p, q}\left(a \alpha_{p}(1) \otimes_{A} b \alpha_{q}(1)\right):=a \alpha_{p}(b) \alpha_{p q}(1)$ (see [38]). Thus the covariance algebra of such a product system may be viewed as the crossed product of $A$ under $V$, generalising Exel's construction to interaction groups satisfying $V_{q^{-1}} V_{p^{-1}}=V_{q^{-1} p^{-1}}$ that are not necessarily nondegenerate. For instance, the product system built in the previous subsection fits into this setting, where $V_{g}\left(\chi_{S}\right):=\chi_{g S \cap P}$ for all $S \in \mathcal{J}$ and $g \in G$. 


\section{Appendix A}

\section{General theory of Hilbert modules}

In this appendix, we recall some basic aspects of the theory of Hilbert modules. We state some results that were needed in the main text of this work. This appendix is based on [36] and [52].

\section{A.1 Adjointable operators on Hilbert modules}

Definition A.1.1. Let $\mathcal{E}$ be a complex vector space and $A$ a $\mathrm{C}^{*}$-algebra. We say that $\mathcal{E}$ is a (right) pre-Hilbert $A$-module if $\mathcal{E}$ is a right $A$-module equipped with a map $\langle\cdot \mid \cdot\rangle: \mathcal{E} \times \mathcal{E} \rightarrow A$, that is linear in the second variable and conjugate-linear in the first, satisfying for all $\xi, \eta, \zeta \in \mathcal{E}$ and $a \in A$,

(i) $\langle\xi \mid \eta a\rangle=\langle\xi \mid \eta\rangle a$;

(ii) $\langle\xi \mid \eta\rangle^{*}=\langle\eta \mid \xi\rangle$;

(iii) $\langle\xi \mid \xi\rangle \geq 0$ in $A$;

(iv) $\langle\xi \mid \xi\rangle=0 \Rightarrow \xi=0$

The map $\langle\cdot \mid \cdot\rangle$ is referred to as inner product.

Remark A.1.2. The axioms (i) and (iii) imply that $\langle\xi a \mid \eta\rangle=a^{*}\langle\xi \mid \eta\rangle$. In particular, the closure of

$$
\langle\mathcal{E} \mid \mathcal{E}\rangle=\operatorname{span}\{\langle\xi \mid \eta\rangle \mid \xi, \eta \in \mathcal{E}\}
$$

is a closed ideal in $A$.

A left pre-Hilbert $A$-module is defined in a similar way. We require the inner product to be $A$-linear in the first variable and thus conjugate-linear in the second. We use the notation $\langle\langle\cdot \mid \cdot\rangle\rangle$ for the inner product of a left pre-Hilbert $A$-module.

A pre-Hilbert $A$-module is called full if the ideal $\langle\mathcal{E} \mid \mathcal{E}\rangle$ is dense in $A$.

Lemma A.1.3 (Cauchy-Schwarz inequality). Let $\mathcal{E}$ be a pre-Hilbert A-module and $\xi, \eta \in \mathcal{E}$. Then

$$
\langle\xi \mid \eta\rangle^{*}\langle\xi \mid \eta\rangle \leq\|\langle\xi \mid \xi\rangle\|\langle\eta \mid \eta\rangle \text {. }
$$

Corollary A.1.4. If $\mathcal{E}$ is a pre-Hilbert A-module, then

$$
\|\cdot\|: \xi \mapsto\|\xi\|:=\|\langle\xi \mid \xi\rangle\|^{\frac{1}{2}}
$$

is a norm on $\mathcal{E}$ for which $\|\xi a\| \leq\|\xi\|\|a\|$. Moreover,

$$
\mathcal{E}\langle\mathcal{E} \mid \mathcal{E}\rangle=\operatorname{span}\{\xi\langle\eta \mid \zeta\rangle \mid \xi, \eta, \zeta \in \mathcal{E}\}
$$

is dense in $\mathcal{E}$.

Definition A.1.5. A Hilbert $A$-module is a pre-Hilbert $A$-module $\mathcal{E}$ that is complete in the norm coming from the $A$-valued inner product. 


\section{A. GENERAL THEORY OF HILBERT MODULES}

Example A.1.6. A Hilbert space $\mathcal{H}$ may be viewed as a Hilbert $\mathbb{C}$-module. It is also a left Hilbert $\mathbb{K}(\mathcal{H})$-module with left inner product given by

$$
\langle\langle\xi \mid \eta\rangle\rangle:=|\xi\rangle\langle\eta|
$$

where $|\xi\rangle\langle\eta|$ denotes the compact operator on $\mathcal{H}$ determined by the vectors $\xi$ and $\eta$. That is, $|\xi\rangle\langle\eta|(\zeta)=\xi\langle\eta \mid \zeta\rangle$ for all $\zeta \in \mathcal{H}$.

Example A.1.7. A $\mathrm{C}^{*}$-algebra $A$ has a canonical structure of right Hilbert $A$-module with right module action implemented by the multiplication in $A$ and inner product

$$
(a, b) \mapsto a^{*} b .
$$

Taking $(a, b) \mapsto a b^{*}$ as inner product, $A$ becomes a left Hilbert $A$-module with left action given by left multiplication. A closed ideal $I \triangleleft A$ may be turned into right and left Hilbert $A$-modules in a similar way.

Example A.1.8 (Direct sum). Let $\mathcal{E}$ and $\mathcal{G}$ be Hilbert $A$-modules. Then $\mathcal{E} \oplus \mathcal{G}$ is a Hilbert $A$-module with right action of $A$ and $A$-valued inner product defined coordinatewise. More generally, given a family of Hilbert $A$-modules $\left(\mathcal{E}_{\lambda}\right)_{\lambda \in \Lambda}$, then the algebraic direct sum $\bigoplus_{\lambda \in \Lambda} \mathcal{E}_{\lambda}$ is a pre-Hilbert $A$-module with the structure defined coordinatewise. Its completion is a Hilbert $A$-module.

Definition A.1.9. Let $\mathcal{E}$ and $\mathcal{G}$ be Hilbert $A$-modules. A map $T: \mathcal{E} \rightarrow \mathcal{G}$ is adjointablel if there exists a map $T^{*}: \mathcal{G} \rightarrow \mathcal{E}$ such that for all $\xi \in \mathcal{E}$ and $\eta \in \mathcal{G}$

$$
\langle T(\xi) \mid \eta\rangle=\left\langle\xi \mid T^{*}(\eta)\right\rangle \text {. }
$$

This is unique if it exists. We say that $T^{*}$ is the adjoint of $T$.

Lemma A.1.10. An adjointable map $T: \mathcal{E} \rightarrow \mathcal{G}$ is A-linear and continuous.

Remark A.1.11. There are continuous $A$-module maps that are not adjointable.

Given Hilbert $A$-modules $\mathcal{E}$ and $\mathcal{G}$, we denote by $\mathbb{B}(\mathcal{E}, \mathcal{G})$ the set of all adjointable operators from $\mathcal{E}$ to $\mathcal{G}$. We write $\mathbb{B}(\mathcal{E})$ in case $\mathcal{E}=\mathcal{G}$.

Proposition A.1.12. If $\mathcal{E}$ is a Hilbert $A$-module, then $\mathbb{B}(\mathcal{E})$ is a $\mathrm{C}^{*}$-algebra with respect to the operator norm.

Corollary A.1.13. Let $\mathcal{E}$ be a Hilbert $A$-module and $T \in \mathbb{B}(\mathcal{E})$. Then, for all $\xi \in \mathcal{E}$,

$$
\langle T(\xi) \mid T(\xi)\rangle \leq\|T\|^{2}\langle\xi \mid \xi\rangle .
$$

We may attach to elements $\xi \in \mathcal{G}$ and $\eta \in \mathcal{E}$ an adjointable operator $\mathcal{E} \rightarrow \mathcal{G}$ defined by

$$
|\xi\rangle\langle\eta|: \zeta \mapsto \xi\langle\eta \mid \zeta\rangle
$$

This is the compact operator determined by $\xi$ and $\eta$. Its adjoint is $|\eta\rangle\langle\xi| \in \mathbb{B}(\mathcal{G}, \mathcal{E})$. The closed linear span of operators of this form is denoted by $\mathbb{K}(\mathcal{E}, \mathcal{G})$. An element of $\mathbb{K}(\mathcal{E}, \mathcal{G})$ is said to be compact. If $\mathcal{E}=\mathcal{G}, \mathbb{K}(\mathcal{E})=\mathbb{K}(\mathcal{E}, \mathcal{E})$ is an ideal of $\mathbb{B}(\mathcal{E})$.

\section{A.2 Morita equivalence}

Definition A.2.1. Let $A$ and $B$ be $\mathrm{C}^{*}$-algebras. An imprimitivity $A, B$-bimodule is an $A, B$-bimodule such that

(i) $\mathcal{E}$ is a full left Hilbert $A$-module and a full right Hilbert $B$-module;

(ii) $\langle\langle\xi \mid \eta\rangle\rangle \zeta=\xi\langle\eta \mid \zeta\rangle$ for all $\xi, \eta, \zeta \in \mathcal{E}$.

Example A.2.2. A $\mathrm{C}^{*}$-algebra $A$ has a canonical structure of imprimitivity $A, A$-bimodule with left $A$-valued inner product $\langle\langle a \mid b\rangle\rangle=a b^{*}$. The right $A$-valued inner product is $\langle a \mid b\rangle=a^{*} b$.

Example A.2.3. A full Hilbert $A$-module $\mathcal{E}$ is an imprimitivity $\mathbb{K}(\mathcal{E}), A$-bimodule. 
Proposition A.2.4. Let $\mathcal{E}$ be an imprimitivity $A, B$-bimodule. Then for all $a \in A, b \in B$ and $\xi, \eta \in \mathcal{E}$,

(i) $\langle\langle\xi b \mid \eta\rangle\rangle=\left\langle\left\langle\xi \mid \eta b^{*}\right\rangle\right\rangle$ and $\langle a \xi \mid \eta\rangle=\left\langle\xi \mid a^{*} \eta\right\rangle$;

(ii) $\langle\langle\xi b \mid \xi b\rangle\rangle \leq\|b\|^{2}\langle\langle\xi \mid \xi\rangle\rangle$ and $\langle\langle a \xi \mid a \xi\rangle\rangle \leq\|a\|^{2}\langle\xi \mid \xi\rangle$.

Corollary A.2.5. Let $\mathcal{E}$ be an imprimitivity $A, B$-bimodule. Then $\|\xi\|_{A}=\|\xi\|_{B}$ for all $\xi \in \mathcal{E}$.

Definition A.2.6. Given $\mathrm{C}^{*}$-algebras $A$ and $B$, we say that $A$ is Morita equivalent to $B$ if there exists an imprimitivity $A, B$-bimodule.

If $A$ is Morita equivalent to $B$, we build an imprimitivity $B, A$-bimodule as follows. Let $\mathcal{E}$ be an imprimitivity $A, B$-bimodule and let $\mathcal{E}^{*}$ be a copy of $\mathcal{E}$ as a set. So an element of $\mathcal{E}^{*}$ is of the form $\xi^{*}$ for a unique $\xi$ in $\mathcal{E}$. We define a structure of $B, A$-bimodule on $\mathcal{E}^{*}$ by

$$
\begin{aligned}
\xi^{*}+\lambda \eta^{*} & :=(\xi+\bar{\lambda} \eta)^{*} \\
b \cdot \xi^{*} & :=\left(\xi b^{*}\right)^{*} \\
\xi^{*} \cdot a & :=\left(a^{*} \xi\right)^{*} .
\end{aligned}
$$

The next proposition implies that Morita equivalence is a symmetric relation.

Proposition A.2.7. Let $\mathcal{E}$ be an imprimitivity $A, B$-bimodule. Then $\mathcal{E}^{*}$ is an imprimitivity $B, A$-bimodule with inner products given by

$$
\left\langle\left\langle\xi^{*} \mid \eta^{*}\right\rangle\right\rangle:=\langle\xi \mid \eta\rangle, \quad \text { and } \quad\left\langle\xi^{*} \mid \eta^{*}\right\rangle:=\langle\langle\xi \mid \eta\rangle\rangle, \quad \text { for all } \xi^{*}, \eta^{*} \in \mathcal{E}^{*} .
$$

We call $\mathcal{E}^{*}$ the adjoint of $\mathcal{E}$.

Let $\mathcal{E}$ be a Hilbert $A$-module and $\mathcal{G}$ a Hilbert $B$-module. Let $\psi: A \rightarrow \mathbb{B}(\mathcal{G})$ is a *-homomorphism. We will see that there is a Hilbert $B$-module $\mathcal{E} \otimes_{A} \mathcal{G}$ built out of the algebraic tensor product $\mathcal{E} \odot_{A} \mathcal{G}$.

The right action of $B$ on $\mathcal{E} \otimes_{\psi} \mathcal{G}$ is defined on an elementary tensor by

$$
(\xi \otimes \eta) \cdot b:=\xi \otimes \eta b .
$$

Proposition A.2.8. Let $\mathcal{E}$ be a Hilbert $A$-module and $\mathcal{G}$ be a Hilbert $B$-module. Let $\psi: A \rightarrow \mathbb{B}(\mathcal{G})$ be $a^{*}$-homomorphism. Then $\mathcal{E} \odot_{A} \mathcal{G}$ is a pre-Hilbert B-module. On elementary tensors the inner product is given by

$$
\left\langle\xi_{1} \otimes \eta_{1} \mid \xi_{2} \otimes \eta_{2}\right\rangle=\left\langle\eta_{1} \mid \psi\left(\left\langle\xi_{1} \mid \xi_{2}\right\rangle\right) \eta_{2}\right\rangle,
$$

where $\xi_{1}, \xi_{2} \in \mathcal{E}$ and $\eta_{1}, \eta_{2} \in \mathcal{G}$.

Let $\mathcal{E}$ be an imprimitivity $A, B$-bimodule. If $C$ is a $\mathrm{C}^{*}$-algebra and $\mathcal{G}$ is an imprimitivity $B, C$-bimodule, $B$ acts by adjointable operators on $\mathcal{G}$ by Proposition A.2.4 We will denote the corresponding tensor product of Hilbert modules simply by $\mathcal{E} \otimes_{B} \mathcal{G}$.

Proposition A.2.9. Let $\mathcal{E}$ be an imprimitivity $A, B$-bimodule and $\mathcal{G}$ an imprimitivity $B, C$-bimodule. Then $\mathcal{E} \otimes_{B} \mathcal{G}$ is an imprimitivity $A, C$-bimodule with the left action of $A$ given by $a \cdot(\xi \otimes \eta):=a \xi \otimes \eta$ and left A-valued inner product defined on elementary tensors by

$$
\left\langle\left\langle\xi_{1} \otimes \eta_{1} \mid \xi_{2} \otimes \eta_{2}\right\rangle\right\rangle=\left\langle\left\langle\xi_{1}\left\langle\left\langle\eta_{1} \mid \eta_{2}\right\rangle\right\rangle_{B} \mid \xi_{2}\right\rangle\right\rangle_{A},
$$

where $\xi_{1}, \xi_{2} \in \mathcal{E}$ and $\eta_{1}, \eta_{2} \in \mathcal{G}$. In particular, Morita equivalence is a transitive relation.

We call $\mathcal{E} \otimes_{B} \mathcal{G}$ the internal tensor product.

Proposition A.2.10. Morita equivalence is an equivalence relation amongst $\mathrm{C}^{*}$-algebras.

Given a $\mathrm{C}^{*}$-algebra $A$, we let $\mathcal{I}(A)$ be the set of all closed two-sided ideals of $A$. This has a lattice structure with the partial order given by inclusion. The next theorem is [52, Theorem 3.22].

Theorem A.2.11 (Rieffel correspondence). Let $\mathcal{E}$ be an imprimitivity $A, B$-bimodule. There are lattice isomorphisms among $\mathcal{I}(A), \mathcal{I}(B)$ and the lattice of closed $A, B$-submodules of $\mathcal{E}$. The isomorphisms are given as follows: 
(i) An ideal $J \in \mathcal{I}(B)$ produces an $A, B$-bimodule $\mathcal{G}_{J}$ given by

$$
\mathcal{G}_{J}=\{\xi \in \mathcal{E} \mid\langle\eta \mid \xi\rangle \in J \text { for all } \eta \in \mathcal{E}\}
$$

(ii) If $\mathcal{G}$ is a closed $A, B$-submodule of $\mathcal{E}$, it gives rise to closed two-sided ideals in $A$ and $B$, respectively, by setting

$$
\left.I_{A}=\overline{\operatorname{span}}\{\langle\xi \mid \eta\rangle\rangle \mid \xi \in \mathcal{G}, \eta \in \mathcal{E}\right\} \quad \text { and } \quad J_{B}=\overline{\operatorname{span}}\{\langle\eta \mid \xi\rangle \mid \xi \in \mathcal{G}, \eta \in \mathcal{E}\}
$$

(iii) An ideal $I \in \mathcal{I}(A)$ gives a closed $A, B$-submodule of $\mathcal{E}$ by

$$
{ }_{I} \mathcal{G}=\{\xi \in \mathcal{E} \mid\langle\langle\xi \mid \eta\rangle\rangle \in I \text { for all } \eta \in \mathcal{E}\} .
$$




\section{Appendix B}

\section{Bicategories}

We recall some basic definitions from bicategory theory, following 9 , 27. We also give a few examples with the main chapters in mind.

\section{B.1 Bicategories, homomorphisms and transformations}

Definition B.1.1. A bicategory $\mathcal{B}$ consists of the following data:

- a set of objects ob $\mathcal{B}$;

- a category $\mathcal{B}(x, y)$ for each pair of objects $(x, y)$; objects of $\mathcal{B}(x, y)$ are called arrows (or morphisms) from $x$ to $y$, and arrows in $\mathcal{B}(x, y)$ are called 2-arrows (or 2-morphisms); the category structure on $\mathcal{B}(x, y)$ gives us a unit 2-arrow $1_{f}$ on each arrow $f: x \rightarrow y$, and a vertical composition of 2-arrows: $w_{0}: f_{0} \Rightarrow f_{1}$ and $w_{1}: f_{1} \Rightarrow f_{2}$ compose to a 2-arrow $w_{1} \cdot w_{0}: f_{0} \Rightarrow f_{2}$;

- composition functors

$$
\circ: \mathcal{B}(y, z) \times \mathcal{B}(x, y) \rightarrow \mathcal{B}(x, z)
$$

for each triple of objects $(x, y, z)$; this contains a horizontal composition of 2-arrows as displayed below:

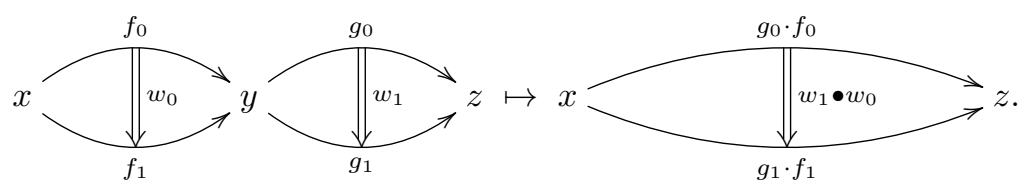

- a unit arrow $1_{x} \in \mathcal{B}(x, x)$ for each $x$;

- natural invertible 2-arrows (unitors) $r_{f}: f \cdot 1_{x} \Rightarrow f$ and $l_{f}: 1_{y} \cdot f \Rightarrow f$ for all $f \in \mathcal{B}(x, y)$;

- natural isomorphisms

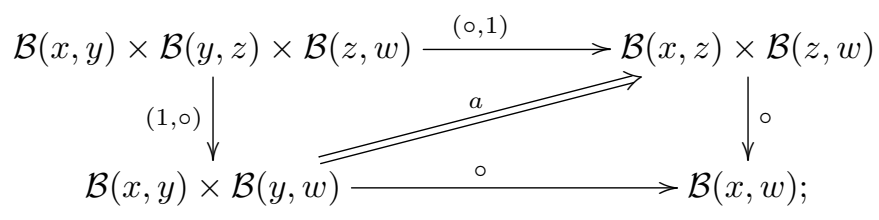

that is, natural invertible 2-arrows, called associators,

$$
a\left(f_{1}, f_{2}, f_{3}\right):\left(f_{3} \cdot f_{2}\right) \cdot f_{1} \underset{\simeq}{\Rightarrow} f_{3} \cdot\left(f_{2} \cdot f_{1}\right)
$$

where $f_{1}: x \rightarrow y, f_{2}: y \rightarrow z$ and $f_{3}: z \rightarrow w$. 


\section{B. BICATEGORIES}

This data must make the following diagrams commute:
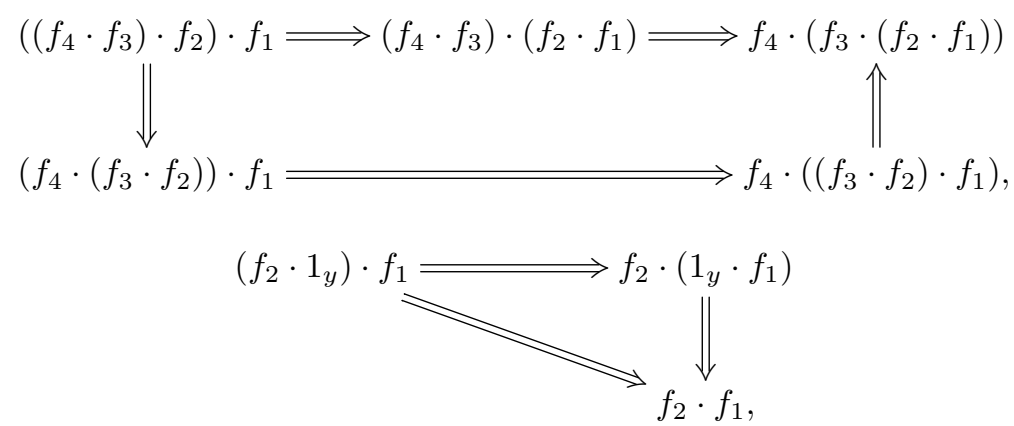

where $f_{1}, f_{2}, f_{3}$, and $f_{4}$ are composable arrows, and the 2-arrows are associators and unitors and horizontal products of them with unit 2-arrows.

We write "." or nothing for vertical products and "•" for horizontal products.

Example B.1.2. Categories form a bicategory Cat with functors as arrows and natural transformations as 2-arrows. Here the composition of morphisms is strictly associative and unital, that is, Cat is even a 2-category.

Example B.1.3. A category $\mathcal{C}$ may be regarded as a bicategory in which the categories $\mathcal{C}(x, y)$ have only identity arrows.

Example B.1.4. The correspondence bicategory $\mathfrak{C}$ is defined in $\left[14\right.$ as the bicategory with $\mathrm{C}^{*}$-algebras as objects, correspondences as arrows, and correspondence isomorphisms as 2-arrows. The unit arrow $1_{A}$ on a $\mathrm{C}^{*}$-algebra $A$ is $A$ viewed as a Hilbert $A$-bimodule in the canonical way. The $A, B$-bimodule structure on $\mathcal{F}$ provides the unitors $A \otimes_{A} \mathcal{F} \Rightarrow \mathcal{F}$ and $\mathcal{F} \otimes_{B} B \Rightarrow \mathcal{F}$ for a correspondence $\mathcal{F}: A \leadsto B$. The associators $\left(\mathcal{E} \otimes_{A} \mathcal{F}\right) \otimes_{B} \mathcal{G} \Rightarrow \mathcal{E} \otimes_{A}\left(\mathcal{F} \otimes_{B} \mathcal{G}\right)$ are the obvious isomorphisms.

Definition B.1.5. Let $\mathcal{B}, \mathcal{C}$ be bicategories. A homomorphism $F: \mathcal{B} \rightarrow \mathcal{C}$ consists of

- a map $F:$ ob $\mathcal{B} \rightarrow$ ob $\mathcal{C}$ between the object sets;

- functors $F_{x, y}: \mathcal{B}(x, y) \rightarrow \mathcal{C}\left(F^{0}(x), F^{0}(y)\right)$ for all $x, y \in$ ob $\mathcal{B}$;

- natural transformations

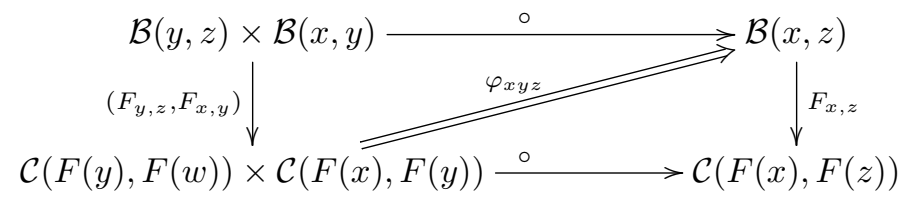

for all triples $x, y, z$ of objects of $\mathcal{B}$; explicitly, these are natural 2-arrows

$$
\varphi\left(f_{1}, f_{2}\right): F_{y, z}\left(f_{2}\right) \cdot F_{x, y}\left(f_{1}\right) \Rightarrow F_{x, z}\left(f_{2} \cdot f_{1}\right) ;
$$

- 2-arrows $\varphi_{x}: 1_{F(x)} \Rightarrow F_{x, x}\left(1_{x}\right)$ for all objects $x$ of $\mathcal{B}$.

This data must make the following diagrams commute:

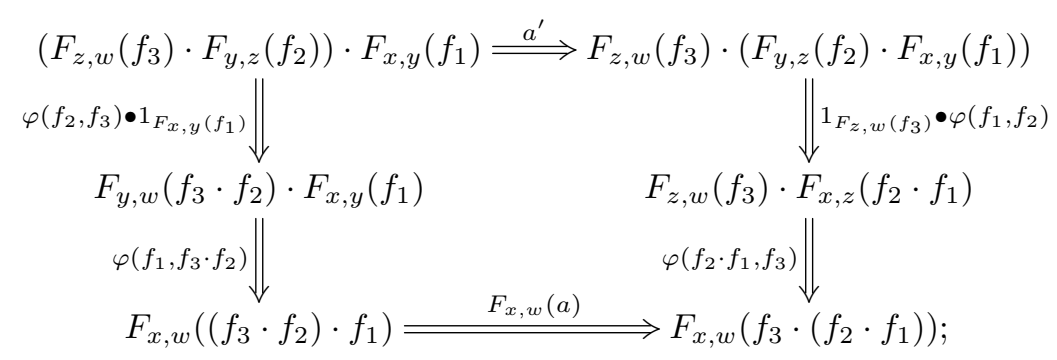




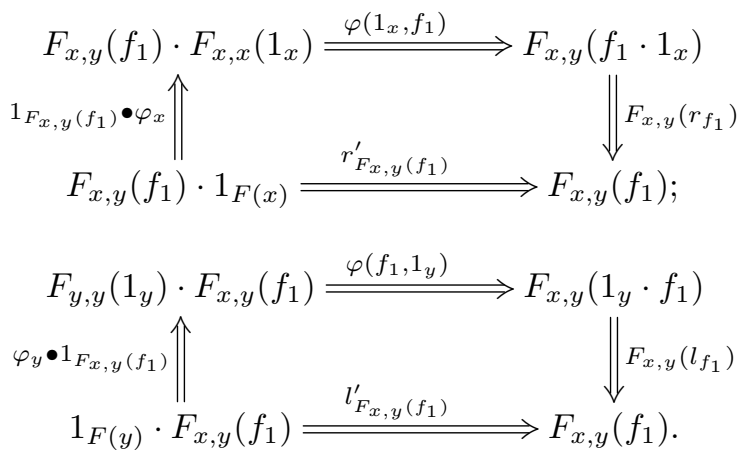

Example B.1.9. A semigroup $P$ may be viewed as a category with one object and $P$ as its set of arrows. It may be viewed as a bicategory as well as in Example B.1.3. A homomorphism from $P$ to $\mathfrak{C}$ is equivalent to an essential product system $\left(A,\left(\mathcal{E}_{p}\right)_{p \in P^{\circ p}}, \mu\right)$ over $P^{\text {op }}$ as defined by Fowler 26 . The condition (B.1.6) says that the multiplication maps $\mu_{p, q}: \mathcal{E}_{p} \otimes_{A} \mathcal{E}_{q} \stackrel{\simeq}{\rightarrow} \mathcal{E}_{q p}$ are associative. The conditions (B.1.7) and B.1.8 mean that $\mu_{1, p}(a \otimes \xi)=\varphi_{p}(a) \xi$ and $\mu_{p, 1}(\xi \otimes a)=\xi a$ for $a \in A, \xi \in \mathcal{E}_{p}$.

A morphism $f: x \rightarrow y$ in a bicategory $\mathcal{B}$ induces functors

$$
f_{*}: \mathcal{B}(c, x) \rightarrow \mathcal{B}(c, y), \quad f^{*}: \mathcal{B}(y, c) \rightarrow \mathcal{B}(x, c)
$$

for $c \in$ ob $\mathcal{B}$ by composing arrows with $f$ and composing 2-arrows horizontally with $1_{f}$ on one side (this is also called whiskering with $f$ ).

Definition B.1.10. Let $F, G: \mathcal{B} \rightrightarrows \mathcal{C}$ be homomorphisms. A transformation $\alpha: F \Rightarrow G$ consists of

- morphisms $\alpha_{x}: F(x) \rightarrow G(x)$ for all $x \in$ ob $\mathcal{B}$;

- natural transformations

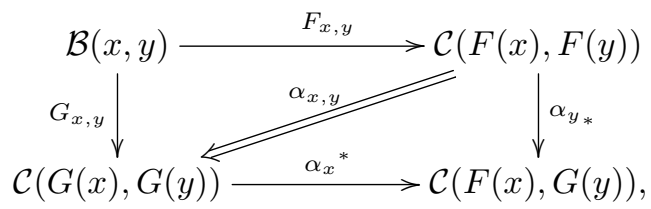

that is, 2-arrows $\alpha_{x, y}(f): \alpha_{y} F_{x, y}(f) \Rightarrow G_{x, y}(f) \alpha_{x}$ for all $x, y \in$ ob $\mathcal{B}$.

This data must make the following diagrams commute:

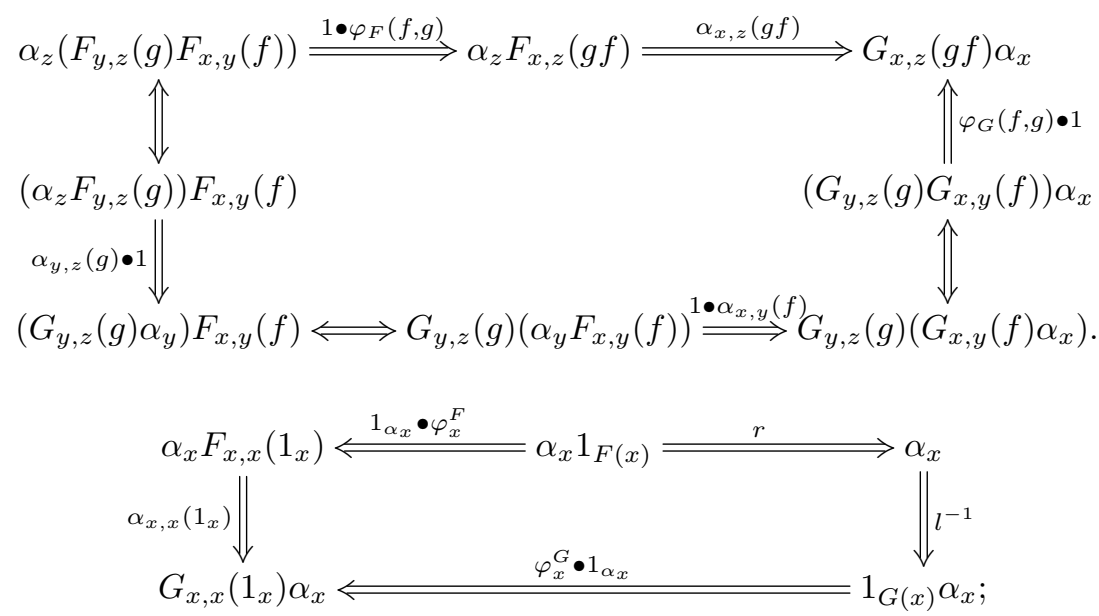

Example B.1.11. Let $G$ be a group. A transformation between homomorphisms $G \rightarrow \mathfrak{C}$ consists of a correspondence $\mathcal{F}: A \leadsto B$ and isomorphisms $\alpha_{s}: \mathcal{E}_{s} \otimes_{A} \mathcal{F} \simeq \mathcal{F} \otimes_{B} \mathcal{G}_{s}$ so that the following diagrams 


\section{B. BICATEGORIES}

commute for all $s, t \in G$ :

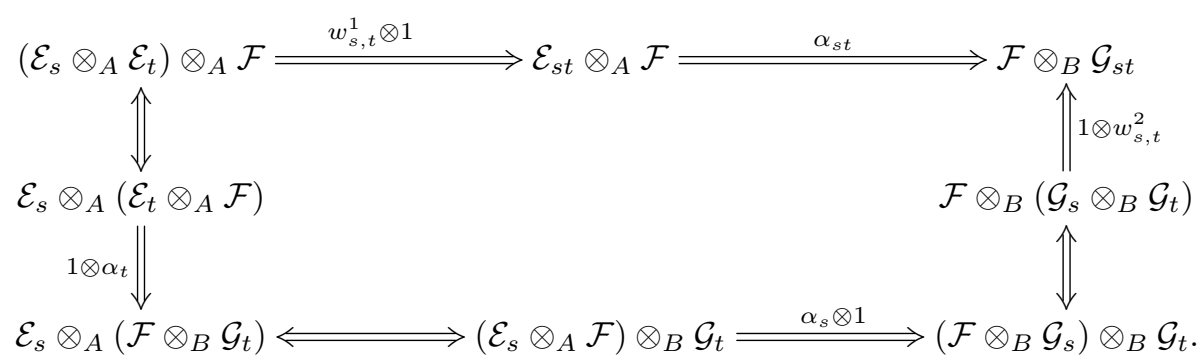

This is called a correspondence of Fell bundles (see [14, Proposition 3.23]).

Definition B.1.12. Let $\alpha, \beta: F \Rightarrow G$ be transformations between homomorphisms. A modification $\Delta: \alpha \Rightarrow \beta$ is a family of 2-arrows $\Delta_{x}: \alpha_{x} \Rightarrow \beta_{x}$ such that for every 2-arrow $w: f_{1} \Rightarrow f_{2}$ for arrows $f_{1}, f_{2}: x \rightarrow y$, the following diagram commutes:

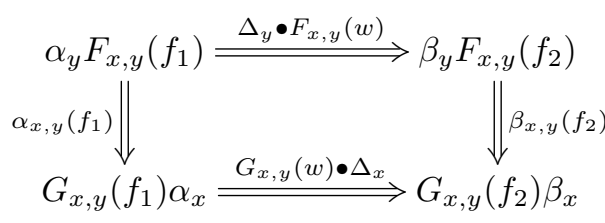




\section{Bibliography}

[1] Beatriz Abadie, Søren Eilers, and Ruy Exel, Morita equivalence for crossed products by Hilbert $C^{*}$-bimodules, Trans. Amer. Math. Soc. 350 (1998), no. 8, 3043-3054, doi: 10.1090/S0002-9947-98-02133-3. MR 1467459

[2] Fernando Abadie and Damián Ferraro, Equivalence of Fell bundles over groups (2017). arXiv: 1711.02577v1

[3] Sriwulan Adji, Marcelo Laca, May Nilsen, and Iain Raeburn, Crossed products by semigroups of endomorphisms and the Toeplitz algebras of ordered groups, Proceedings of the American Mathematical Society 122 (1994), no. 4, 1133-1141, doi: 10.2307/2161182 MR 1215024

[4] Sriwulan Adji, Invariant ideals of crossed products by semigroups of endomorphisms, International Conference on Functional Analysis and Global Analysis (1997), Functional analysis and global analysis: Proceedings of the conference held in Manila, Philippines (T. Sunada and P. W. Sy), Singapore: Springer, 1996.

[5] Suliman Albandik and Ralf Meyer, Product systems over Ore monoids, Doc. Math. 20 (2015), 1331-1402, available at http://www.math.uni-bielefeld.de/documenta/vol-20/38.html. MR 3452185

[6] _ Colimits in the correspondence bicategory, Münster J. Math. 9 (2016), 51-76, doi: 10.17879/45209432019 MR 3549542

[7] William Arveson, Continuous analogues of Fock space, Mem. Amer. Math. Soc. 80 (1989), no. 409, iv+66, doi: 10.1090/memo/0409 MR 987590

[8] Saad Baaj and Georges Skandalis, $C^{*}$-algèbres de Hopf et théorie de Kasparov équivariante, K-Theory 2 (1989), no. 6, 683-721, doi: 10.1007/BF00538428. MR 1010978

[9] Jean Bénabou, Introduction to bicategories, Reports of the Midwest Category Seminar, Springer, Berlin, 1967, pp. 1-77, doi: 10.1007/BFb0074299 MR 0220789

[10] Nathanial P. Brown and Narutaka Ozawa, $C^{*}$-algebras and finite-dimensional approximations, Graduate Studies in Mathematics, vol. 88, Amer. Math. Soc., Providence, RI, 2008. MR 2391387

[11] Nathan Brownlowe and Iain Raeburn, Exel's crossed product and relative Cuntz-Pimsner algebras, Mathematical Proceedings of the Cambridge Philosophical Society 141 (2006), no. 3, 497-508, doi: 10.1017/S030500410600956X MR MR2281412

[12] Alcides Buss and Ralf Meyer, Inverse semigroup actions on groupoids, Rocky Mountain J. Math. 47 (2017), no. 1, 53-159, doi: 10.1216/RMJ-2017-47-1-53 MR $\mathbf{3 6 1 9 7 5 8}$

[13] Alcides Buss, Ralf Meyer, and Chenchang Zhu, Non-Hausdorff symmetries of C*-algebras, Math. Ann. 352 (2012), no. 1, 73-97, doi: 10.1007/s00208-010-0630-3. MR 2885576

[14] - A higher category approach to twisted actions on $\mathrm{C}^{*}$-algebras, Proc. Edinb. Math. Soc. (2) 56 (2013), no. 2, 387-426, doi: 10.1017/S0013091512000259 MR $\mathbf{3 0 5 6 6 5 0}$

[15] Toke M. Carlsen, Nadia S. Larsen, Aidan Sims, and Sean T. Vittadello, Co-universal algebras associated to product systems, and gauge-invariant uniqueness theorems, Proc. Lond. Math. Soc. (3) 103 (2011), no. 4, 563-600, doi: 10.1112/plms/pdq028 MR 2837016

[16] Lisa Orloff Clark, Astrid an Huef, and Iain Raeburn, Phase transitions on the Toeplitz algebras of Baumslag-Solitar semigroups, Indiana Univ. Math. J. 65 (2016), no. 6, 2137-2173, doi: 10.1512/iumj.2016.65.5934 MR 3595491

[17] John Crisp and Marcelo Laca, On the Toeplitz algebras of right-angled and finite-type Artin groups, Journal of the Australian Mathematical Society 72 (2002), no. 2, 223-246, doi: 10.1017/S1446788700003876 $\mid$ MR 1887134

[18] Siegfried Echterhoff, Steven P. Kaliszewski, John Quigg, and Iain Raeburn, A categorical approach to imprimitivity theorems for $C^{*}$-dynamical systems, Mem. Amer. Math. Soc. 180 (2006), no. 850, viii+169, doi: 10.1090/memo/0850 MR 2203930

[19] Ruy Exel, Partial representations and amenable Fell bundles over free groups, Pacific J. Math. 192 (2000), no. 1, 39-63, doi: 10.2140/pjm.2000.192.39 MR 1741030

[20] - Circle actions on $C^{*}$-algebras, partial automorphisms, and a generalized Pimsner-Voiculescu exact sequence, J. Funct. Anal. 122 (1994), no. 2, 361-401, doi: 10.1006/jfan.1994.1073. MR 1276163

[21] _ A new look at the crossed-product of a $C^{*}$-algebra by an endomorphism, Ergodic Theory Dynam. Systems 23 (2003), no. 6, 1733-1750, doi: 10.1017/S0143385702001797 MR 2032486

[22] _ A new look at the crossed product of a $C^{*}$-algebra by a semigroup of endomorphisms, Ergodic Theory Dynam. Systems 28 (2008), no. 3, 749-789, doi: 10.1017/S0143385707000302 MR 2422015

[23] _ Partial dynamical systems, Fell bundles and applications, Mathematical Surveys and Monographs, vol. 224, Amer. Math. Soc., Providence, RI, 2017. 
[24] Thomas M. Fiore, Pseudo limits, biadjoints, and pseudo algebras: categorical foundations of conformal field theory, Mem. Amer. Math. Soc. 182 (2006), no. 860, x+171, doi: 10.1090/memo/0860 arXiv: math/0408298 MR 2229946

[25] Gerald B. Folland, A course in abstract harmonic analysis, Studies in Advanced Mathematics, CRC Press, Boca Raton, FL, 1995. MR 1397028

[26] Neal J. Fowler, Discrete product systems of Hilbert bimodules, Pacific J. Math. 204 (2002), no. 2, 335-375, doi: 10.2140/pjm.2002.204.335 MR 1907896

[27] John W. Gray, Formal category theory: adjointness for 2-categories, Lecture Notes in Mathematics, vol. 391, Springer-Verlag, Berlin-New York, 1974. doi: 10.1007/BFb0061280 MR 0371990

[28] Nick Gurski, Biequivalences in tricategories, Theory Appl. Categ. 26 (2012), No. 14, 349-384, available at http: //www.tac.mta.ca/tac/volumes/26/14/26-14abs.html MR 2972968

[29] Takeshi Katsura, On $C^{*}$-algebras associated with $C^{*}$-correspondences, J. Funct. Anal. 217 (2004), no. 2, 366-401, doi: $10.1016 /$ j.jfa.2004.03.010 MR 2102572

[30] _ A class of $C^{*}$-algebras generalizing both graph algebras and homeomorphism $C^{*}$-algebras. I. Fundamental results, Trans. Amer. Math. Soc. 356 (2004), no. 11, 4287-4322, doi: 10.1090/S0002-9947-04-03636-0 MR 2067120

[31] _ Ideal structure of $C^{*}$-algebras associated with $C^{*}$-correspondences, Pacific J. Math. 230 (2007), no. 1, 107-145, doi: 10.2140/pjm.2007.230.107 MR 2413377

[32] Bartosz Kosma Kwaśniewski, Covariance algebra of a partial dynamical system, Cent. Eur. J. Math. 3 (2005), no. 4, 718-765, doi: 10.2478/BF02475628 MR 2171670

[33] Bartosz Kosma Kwaśniewski and Ralf Meyer, Aperiodicity, topological freeness and pure outerness: from group actions to Fell bundles, Studia Math. (2016), accepted. arXiv: 1611.06954

[34] Bartosz Kosma Kwaśniewski and Wojciech Szymański, Topological aperiodicity for product systems over semigroups of Ore type, J. Funct. Anal. 270 (2016), no. 9, 3453-3504, doi: 10.1016/j.jfa.2016.02.014. MR 3475461

[35] Marcelo Laca and Iain Raeburn, Semigroup Crossed Products and the Toeplitz Algebras of Nonabelian Groups, Journal of Functional Analysis 139 (1996), no. 2, 415-440, doi: 10.1006/jfan.1996.0091 MR 1402771

[36] E. Christopher Lance, Hilbert $C^{*}$-modules, London Mathematical Society Lecture Note Series, vol. 210, Cambridge University Press, Cambridge, 1995. doi: 10.1017/CBO9780511526206 MR 1325694

[37] Nadia S. Larsen, Crossed products by semigroups of endomorphisms and groups of partial automorphisms, Canad. Math. Bull. 46 (2003), no. 1, 98-112, doi: 10.4153/CMB-2003-010-2 MR 1955617

[38] _ Crossed products by abelian semigroups via transfer operators, Ergodic Theory Dynam. Systems 30 (2010), no. 4, 1147-1164, doi: 10.1017/S0143385709000509 MR 2669415

[39] Xin Li, Semigroup C*-algebras and amenability of semigroups, J. Funct. Anal. 262 (2012), no. 10, 4302-4340, doi: 10.1016/j.jfa.2012.02.020 MR 2900468

[40] Ralf Meyer and Camila F. Sehnem, A bicategorical interpretation for relative Cuntz-Pimsner algebras (2017), eprint. arXiv: 1708.03471

[41] Paul S. Muhly and Baruch Solel, Tensor algebras over $C^{*}$-correspondences: representations, dilations, and $C^{*}$-envelopes, J. Funct. Anal. 158 (1998), no. 2, 389-457, doi: 10.1006/jfan.1998.3294 MR 1648483

[42] $\_$On the Morita equivalence of tensor algebras, Proc. London Math. Soc. (3) 81 (2000), no. 1, 113-168, doi 10.1112/S0024611500012405 MR 1757049

[43] Gerard J. Murphy, C*-algebras and Operator Theory, Academic Press, 1990.

[44] _ Crossed products of $C^{*}$-algebras by semigroups of automorphisms, Proc. London Math. Soc. (3) 68 (1994), no. 2, 423-448, doi: $10.1112 / \mathrm{plms} / \mathrm{s} 3-68.2 .423$ MR 1253510

[45] _ , $C^{*}$-Algebras Generated by Commuting Isometries, Rocky Mountain J. Math. 26 (1996), no. 1, 237-267, doi: $10.1216 / \mathrm{rmjm} / 1181072114$. MR 1386163

[46] Chi-Keung Ng, Discrete coactions on $\mathrm{C}^{*}$-algebras, Journal of the Australian Mathematical Society, 1996, pp. 118-127, doi: $10.1017 /$ S1446788700037423 MR $\mathbf{1 3 6 4 5 5 7}$

[47] A. Nica, $C^{*}$-algebras generated by isometries and Wiener-Hopf operators, Journal of Operator Theory 27 (1992), no. 1, 17-52, available at https://www.theta.ro/jot/archive/1992-027-001/1992-027-001-002.pd:AMR 1241114

[48] Mihai V. Pimsner, A class of $C^{*}$-algebras generalizing both Cuntz-Krieger algebras and crossed products by $\mathbf{Z}$, Free probability theory (Waterloo, ON, 1995), Fields Inst. Commun., vol. 12, Amer. Math. Soc., Providence, RI, 1997, pp. 189-212. MR 1426840

[49] John C. Quigg, Full and reduced $C^{*}$-coactions, Math. Proc. Cambridge Philos. Soc. 116 (1994), no. 3, 435-450, doi: 10.1017/S0305004100072728 MR 1291751

[50] , Discrete $C^{*}$-coactions and $C^{*}$-algebraic bundles, J. Austral. Math. Soc. Ser. A 60 (1996), no. 2, 204-221, doi: $10.1017 / \mathrm{S} 1446788700037605$ MR $\mathbf{1 3 7 5 5 8 6}$

[51] Iain Raeburn and Aidan Sims, Product systems of graphs and the Toeplitz algebras of higher-rank graphs, J. Operator Theory 53 (2005), no. 2, 399-429, available at http://www.theta.ro/jot/archive/2005-053-002/ 2005-053-002-010.html MR 2153156

[52] Iain Raeburn and Dana P. Williams, Morita equivalence and continuous-trace $C^{*}$-algebras, Mathematical Surveys and Monographs, vol. 60, Amer. Math. Soc., Providence, RI, 1998. doi: 10.1090/surv/060 MR 1634408

[53] Adam Rennie, David Robertson, and Aidan Sims, Groupoid Fell bundles for product systems over quasi-lattice ordered groups, Math. Proc. Camb. Phil. Soc. 163 (2017), 561-580, doi: 10.1017/S0305004117000202. 
[54] Jürgen Schweizer, Crossed products by $C^{*}$-correspondences and Cuntz-Pimsner algebras, $C^{*}$-Algebras (Münster, 1999), Springer, Berlin, 2000, pp. 203-226, doi: 10.1007/978-3-642-57288-3. MR 1798598

[55] Aidan Sims and Trent Yeend, $C^{*}$-algebras associated to product systems of Hilbert bimodules, J. Operator Theory 64 (2010), no. 2, 349-376, available at http://www.theta.ro/jot/archive/2010-064-002/2010-064-002-005.htm] [MR 2718947

[56] Jack Spielberg, $C^{*}$-algebras for categories of paths associated to the Baumslag-Solitar groups, J. Lond. Math. Soc. (2) 86 (2012), no. 3, 728-754, doi: $10.1112 / \mathrm{jlms} / \mathrm{jds} 025$ MR $\mathbf{3 0 0 0 8 2 8}$

[57] Ross Street, Fibrations in bicategories, Cahiers Topologie Géom. Différentielle 21 (1980), no. 2, 111-160MR 574662 



\section{Camila Fabre Sehnem}

\section{Personal data}

$\begin{array}{ll}\text { Date of birth } & 08.01 .1989 \\ \text { Place of birth } & \text { Criciúma (Brazil) } \\ \text { Gender } & \text { Female }\end{array}$

\section{Academic education and academic degrees}

$08 / 2007-02 / 2012$

$03 / 2012-02 / 2014$

$02 / 2014$

since $04 / 2015$
Mathematics studies at UFSC (Universidade Federal de Santa Catarina) Master studies in mathematics at UFSC Master's degree in mathematics at UFSC, supervisor: Ruy Exel Doctoral studies at Georg-August-Universität Göttingen, supervisor: Ralf Meyer

\section{Scholarships}

2008-2010

2010-2012

$03 / 2012-02 / 2014$

$10 / 2014-03 / 2015$ since $04 / 2015$

\section{Employment}

$03 / 2014-07 / 2014$

\section{Other activities}

01/2012-02-2012

$10 / 2014-03 / 2015$
Stipend from PET (Special Programm of Mathematics)

Scientific initiation stipend from CNPq (National Council for Scientific and Technological Development)

Stipend from CAPES (Coordination for the Improvement of Higher Education Personnel)

Stipend from DAAD (Deutscher Akademischer Austausch Dienst)

Stipend from $\mathrm{CNPq}$
Teacher assistant at IFSC (Instituto Federal de Santa Catarina)

Functional Analysis, summer school at IMPA (Instituto Nacional de Matemática Pura e Aplicada)

Intensive German Language course at Goethe-Institut, Göttingen

\section{Presentations}

- A Classification of Stable Fell bundles, Workshop Groups, Dynamical Systems and C*-algebras, Münster, 2013 (poster presentation).

- A bicategorical interpretation for relative Cuntz-Pimsner algebras, Young Women in $\mathrm{C}^{*}$-algebras, Copenhagen, 2017 (contributed talk).

- A bicategorical interpretation for relative Cuntz-Pimsner algebras, Young Mathematicians in $\mathrm{C}^{*}$-algebras, Copenhagen, 2017 (contributed talk).

- On $\mathrm{C}^{*}$-algebras associated to product systems and semi-saturated Fell bundles, Facets of Irreversibility: inverse semigroups, groupoids, and operator algebras, Oslo, 2017 (contributed talk). 


\section{Events}

- Young Mathematicians in $\mathrm{C}^{*}$-algebras, Copenhagen, 2015.

- Young Mathematicians in $\mathrm{C}^{*}$-algebras, Münster, 2016.

\section{eprints}

[1] Ralf Meyer and Camila F. Sehnem, A bicategorical interpretation for relative Cuntz-Pimsner algebras (2017), eprint. arXiv: 1708.03471

[2] Camila F. Sehnem, On $\mathrm{C}^{*}$-algebras associated to product systems (2018), eprint. arXiv: 1804.10546 